\title{
Detumbling a Non-Cooperative Space Target With UnKNOWN INERTIAL PARAMETERS USING a SPACE MANiPUlator SUbJECTED TO END-EFFECTOR FORCE/TORQUE LIMITS
}

\author{
by \\ Rabindra A. Gangapersaud \\ Bachelor of Engineering, Ryerson University (2013) \\ A dissertation \\ presented to Ryerson University \\ in partial fulfillment of the \\ requirements for the degree of \\ Doctor of Philosophy \\ in the program of \\ Aerospace Engineering
}

Toronto, Ontario, Canada, 2019

(C) Rabindra A. Gangapersaud, 2019 


\section{AUTHOR'S DECLARATION FOR ELECTRONIC SUBMISSION OF A DISSERTATION}

I hereby declare that I am the sole author of this dissertation. This is a true copy of the dissertation, including any required final revisions, as accepted by my examiners.

I authorize Ryerson University to lend this dissertation to other institutions or individuals for the purpose of scholarly research.

I further authorize Ryerson University to reproduce this dissertation by photocopying or by other means, in total or in part, at the request of other institutions or individuals for the purpose of scholarly research.

I understand that my dissertation may be made electronically available to the public. 


\title{
Detumbling a Non-CoOperative Space TARget With UnKNOWn Inertial Parameters Using a Space Manipulator SubJected to End-effector FORCE/TORQUE LIMITS
}

\author{
Rabindra A. Gangapersaud
}

Doctor of Philosophy, Aerospace Engineering, Ryerson University, Toronto (2019)

\begin{abstract}
This study addresses the problem of detumbling a non-cooperative space target, such as a malfunctioning satellite, using a space robot for the purpose of performing on-orbit servicing. The space robot is denoted as the servicer and consists of a satellite base equipped with a robotic manipulator. The formulation of a detumbling control strategy must respect limits on the grasping force and torque at the servicer's end-effector without knowledge of the target's inertial parameters (mass, inertia tensor, location of center of mass). In the literature, prior studies have formulated detumbling strategies under the assumption of accurate knowledge of the target's inertial parameters. However, obtaining accurate estimates of the target's inertial parameters is difficult, and parameter uncertainty may lead to instability and violation of the end-effector force/torque limits. This study will address the problem of detumbling a noncooperative target with unknown but bounded inertial parameters subjected to force/torque limits at the servicer's end-effector.

In this study, two detumbling control strategies are presented. The first detumbling strategy is presented under the assumption that force/torque measurements at the end-effector are available. Detumbling of the target is achieved by applying a reference force/torque to the target that is designed to bring the target's tumbling motion to rest subjected to force/torque limits. To ensure stable detumbling of the target, a robust compensator is designed based on bounds of the target's unknown inertial parameters. Furthermore, once the detumbling process starts, in order to reduce the robust control gains, bounds on the target's unknown inertial parameters
\end{abstract}


are estimated in real-time. The resultant detumbling controller enables the servicer to detumble the target while complying with the target's unknown residual tumbling motion.

The second detumbling control strategy is developed without the need of end-effector's force/torque measurements and takes into account magnitude constraints on servicer's control inputs in the detumbling controller's design. Detumbling is achieved by tracking a desired detumbling trajectory that is delineated subjected to end-effector force/torque limits and requires bounds on the target's inertial parameters. The hyperbolic tangent function is utilized to model the magnitude constraints on the servicer's control inputs, resulting in a system that is non-affine in its control inputs. As a result, an augmented model of the servicer is presented to allow the formulation of the detumbling controller. Using bounds on the target's inertial parameters, robust adaptive control approach is utilized to design the detumbling controller with the backstepping technique in order to track the desired detumbling trajectory and to reject the gained target's momentum.

Numerical simulation studies were conducted for both detumbling control strategies utilizing a servicer equipped with a 7-degree-of-freedom (DOF) manipulator. The results demonstrate that both control strategies are capable of detumbling a non-cooperative target with unknown inertial parameters subjected to force/torque limits. Experiments conducted with a 3-DOF manipulator demonstrate that the design procedure utilized to delineate the desired detumbling trajectory in the second detumbling strategy respects force/torque limits at the endeffector. The study is concluded with a discussion comparing the two proposed detumbling strategies by highlighting their advantages and disadvantages. 


\section{Acknowledgements}

I would like to thank my supervisors, Dr. Guangjun Liu and Dr. Anton de Ruiter, without their support, guidance and encouragement this dissertation would not be possible. I would like to thank the members of the Systems and Control Lab at Ryerson University for all their helpful discussions, and the Examination Committee members, Dr. Krishna Kumar, Dr. Puren Ouyang, Dr. Farrokh Janabi-Sharifi and Dr. Jinjun Shan, for their valuable comments. Finally, I would like to thank my family, especially Annie, for their support, encouragement and patience. 


\section{TABLE OF CONTENTS}

Declaration .............................. ii

Abstract ........................................ iii

Acknowledgements ......................

List of Tables .......................... . ix

List of Figures . . . . . . . . . . . . . . . . . . .

1 Introduction 1

1.1 Background . . . . . . . . . . . . . . . . . . . 1

1.2 Review of Literature on Post-Grasping Phase $\ldots \ldots \ldots \ldots$

1.2 .1 Ideal Scenario . . . . . . . . . . . . . . . . . . . . . . 6

1.2 .2 Identifying the Target's Inertial Parameters $\ldots \ldots \ldots \ldots$. . . . 7

1.2 .3 Post-grasping Phase with Unknown Target $\ldots \ldots \ldots$

1.3 Research Focus and Contributions . . . . . . . . . . . . . . . . . 9

1.4 Organization of Dissertation $\ldots \ldots \ldots \ldots \ldots$

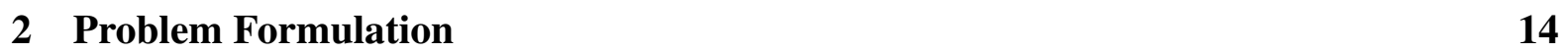

$2.1 \quad$ Post-grasping Phase . . . . . . . . . . . . . . . . . . . . . . . . . . . . 14

2.2 Mathematical Notation . . . . . . . . . . . . . . . . . . . . . 16

2.3 Kinematics of Servicer . . . . . . . . . . . . . . . . . . . . . 17

2.4 Servicer's Momentum Equations $\ldots \ldots$. . . . . . . . . . . . . . . . . . . . . 19

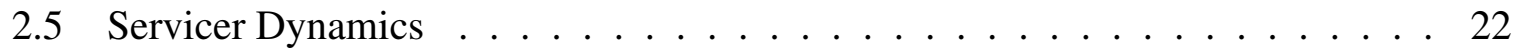

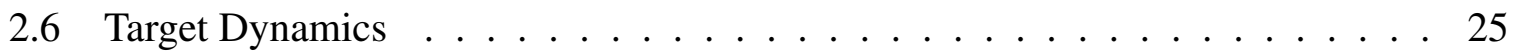

2.7 Combined Servicer-Target Dynamics $\ldots \ldots \ldots \ldots$

2.8 Control Objective $\ldots \ldots \ldots \ldots \ldots \ldots$ 
3 Detumbling Strategy using Force/Torque Measurements 29

$3.1 \quad$ Detumbling Strategy Overview . . . . . . . . . . . . . . . . . . . . . . . . . . 29

3.2 Control of Servicer's Base Attitude . . . . . . . . . . . . . . . . . . . 30

3.3 Control of Servicer's End-effector … . . . . . . . . . . . . . . 31

3.3 .1 Controller Evaluation . . . . . . . . . . . . . . . . . . 32

3.3 .2 Force/Torque Error . . . . . . . . . . . . . . . . 36

3.3 .3 Numerical Simulation . . . . . . . . . . . . . . . . . . . . 37

3.4 Robust Detumbling of Target $\ldots \ldots \ldots$. . . . . . . . . . . . . . . . 44

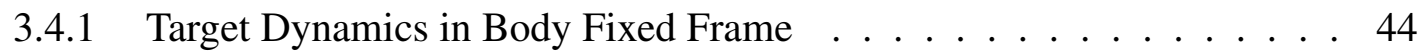

3.4 .2 Modified Detumbling Control … . . . . . . . . . . . . . . 46

3.4 .3 Controller Evaluation. . . . . . . . . . . . . . . . . . . . . 47

3.4 .4 Real-Time Estimation of Bounds on the Target's Inertial Parameters . . 53

3.4 .5 Force/Torque Error . . . . . . . . . . . . . . . . 56

3.4 .6 Implications of Servicer's Force/Torque Limits . . . . . . . . . 56

3.4 .7 Numerical Simulation . . . . . . . . . . . . . . . . . . . 58

3.4.7.1 Detumbling Target with Random Inertial Properties . . . . . 66

3.4.7.2 Effect of $\varepsilon_{\mathrm{r}}$ Parameter on Detumbling Controller's Performance . . . . . . . . . . . . . . . 68

3.5 Conclusions . . . . . . . . . . . . . . . . . . . . . . . . . 69

\begin{tabular}{|lll}
4 & Detumbling Strategy without Force/Torque Measurements & 71
\end{tabular}

4.1 Detumbling Strategy Overview $\ldots \ldots \ldots$. . . . . . . . . . . . . 71

4.1 .1 Delineation of End-effector Detumbling Trajectory . . . . . . . . . 72

4.1 .2 Desired Base Attitude $\ldots \ldots \ldots \ldots$

4.1 .3 Robust Trajectory Tracking Controller. . . . . . . . . . . . . . . . . 79

4.1 .4 Servicer's End-effector Force/Torque Limits. . . . . . . . . . . . . 86

$4.2 \quad$ Numerical Simulation Study of Detumbling Strategy $\ldots \ldots \ldots$. . . . . . . 88

4.2 .1 Detumbling Target with Random Inertial Properties . . . . . . . . . . . 96

4.2 .2 Effect of $\varepsilon_{1}$ Parameter on Detumbling Controller's Performance . . . . 98

4.3 Experimental Evaluation $\ldots \ldots \ldots \ldots 1$

4.4 Conclusions . . . . . . . . . . . . . . . . . . . . . . . . 106 


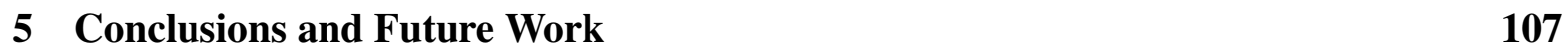

5.1 Summary and Conclusions . . . . . . . . . . . . . . . 107

5.2 Future Work . . . . . . . . . . . . . . . . . . . . . . 110

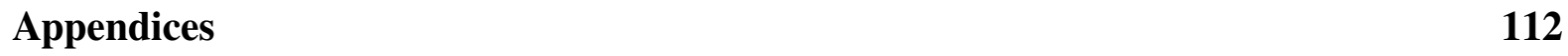

A Description of Servicer and Target Model Parameters Used in Numerical Simula$\begin{array}{ll}\text { tions } & 112\end{array}$

A.1 Model Parameters for Servicer . . . . . . . . . . . . . . . . . . . . . 112

A.2 Model Parameters for Target . . . . . . . . . . . . . . . . . 114

B Upper Bound on $\|\chi\|$ and $\left\|\dot{f}(\mathbf{V})^{\mathrm{d}}\right\|$

\begin{tabular}{lr}
\hline References & 128
\end{tabular} 


\section{List of Tables}

3.1 Target Properties for Numerical Simulation Studies . . . . . . . . . . . . . . 59

3.2 Thruster Firing Logic . . . . . . . . . . . . . . . . . . . . . . 61

$4.1 \quad$ Target's Properties for Numerical Simulation Studies . . . . . . . . . . . . . . 88

4.2 Gains . . . . . . . . . . . . . . . . . . . . 90 


\section{List of Figures}

$1.1 \quad$ Example of space debris removal using net $[13] . \ldots \ldots \ldots$

1.2 JAXA ETS-VII mission on the left [45]. DARPA Orbital Express Program on

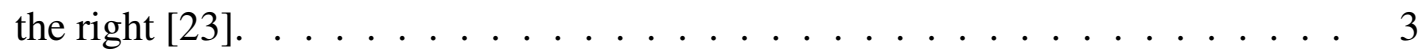

1.3 Phases of satellite capture sequence $[22] . \ldots \ldots \ldots \ldots \ldots$

$2.1 \quad$ Space manipulator and base (servicer) with target $\ldots \ldots \ldots \ldots$. . . . . 17

3.1 End-effector's force and torque profile for Cases 1,2 and 3. . . . . . . . . . . 39

3.2 End-effector's force error profile. Left plots depict force error bound that is computed as per (3.28). Right plots show enlarged force error. . . . . . . . . . 40

3.3 End-effector's torque error profile. Left plots depict torque error bound that is computed as per (3.28). Right plots show enlarged torque error. . . . . . . . . 41

3.4 End-effector's linear and angular velocities for Cases 1,2 and 3. . . . . . . . 42

3.5 Angular momentum distribution for Cases 1,2 and $3 . \ldots \ldots$. . . . . . 43

3.6 Target's kinetic energy for Cases 1,2 and $3 . \ldots \ldots \ldots 4 \ldots$

3.7 Block diagram of the PWPF modulator. . . . . . . . . . . . . . . . 60

3.8 Servicer's base thruster configuration. $\Sigma_{\mathrm{b}}$ denotes body fixed frame located at servicer's base center of mass and coincides with principle axis. $\mathrm{r}=1.15 \mathrm{~m}$. . 60

$3.9 \quad$ Magnitude of end-effector's force/torque profile for Cases 1 and $2 . \quad \ldots \ldots 62$

3.10 End-effector's linear and angular velocities for Cases 1 and 2. . . . . . . . 63

3.11 Magnitude of servicer's control inputs for Case 1. . . . . . . . . . . 63

3.12 Servicer-target system's momentum distribution for Cases 1 and 2. . . . . . . 64

3.13 Target's kinetic energy for Cases 1 and $2 . \ldots \ldots \ldots$. . . . . . . 64

3.14 Time history of $\hat{a}_{i}$ as a percentage of $\bar{a}_{i}$ for Cases 1 and 2. . . . . . . 65

3.15 Servicer's base torque output for Case $2 . \ldots \ldots \ldots 6$ 
3.16 Magnitude of end-effector's force/torque profile as the target's fuel mass varies. 67

3.17 Servicer-target system's momentum distribution profile as the target's fuel mass varies. . . . . . . . . . . . . . . . . . . . 68

3.18 Effect of $\varepsilon_{\mathrm{r}}$ in $\Psi(3.50 \mathrm{a})$ on the end-effector force and torque magnitude profile in the post-grasping phase. . . . . . . . . . . . . . . . . . . . 69

3.19 Effect of $\varepsilon_{\mathrm{r}}$ in $\Psi$ (3.50a) on the magnitude of the servicer's control inputs. . . . 70

4.1 End-effector's desired linear and angular velocity and acceleration. . . . . . . . 91

4.2 End-effector's linear velocity error for Case 1 . . . . . . . . . . . . . . . . . 91

4.3 End-effector's angular velocity error for Case 1 . . . . . . . . . . . . . 92

4.4 Servicer's base angular velocity error for Case 1 . . . . . . . . . . . . . . . . 92

4.5 End-effector's force/torque magnitude and force/torque bound determined from $(4.53)$ for Case 1 . . . . . . . . . . . . . . . . . . . . 93

4.6 Servicer's manipulator and base attitude control input profile for Case 1, Gain Set 3, where $\left\{\mathbf{U}_{\mathrm{M}}\right\}_{i}=8 \mathrm{Nm}$ for $i=1,2,3, \ldots 7$ (manipulator) and $\left\{\mathbf{U}_{\mathrm{M}}\right\}_{i}=6 \mathrm{Nm}$ for $i=8,9,10$ (base attitude control). . . . . . . . . . . . . . . . . . . 93

$4.7 \quad$ Servicer-target system's momentum distribution for Case 1. . . . . . . . . . . . 94

4.8 End-effector's force/torque magnitude and force/torque bound determined from (4.53) for Cases 2. . . . . . . . . . . . . . . . . . . . . 95

4.9 End-effector's linear and angular velocity profile for Case $2 . \quad \ldots . .95$

4.10 Servicer's base torque output for Case 2. . . . . . . . . . . . . . 96 96

4.11 Magnitude of end-effector's force/torque profile as the target's fuel mass is randomly selected. . . . . . . . . . . . . . . . . . . 97

4.12 Servicer-target system's momentum distribution profile as the target's fuel mass is randomly selected. . . . . . . . . . . . . . . . . . . . 98

4.13 Plot of $\tanh (t / \varepsilon)$ for different values of $\varepsilon$. . . . . . . . . . . . . . . 99

4.14 Effect of $\varepsilon_{1}$ in $\psi_{\mathrm{P} 1}$ and $\psi_{\mathrm{P} 2}$ on the end-effector force and torque magnitude profile in the post-grasping phase. . . . . . . . . . . . . . . 100

4.15 Effect of $\varepsilon_{1}$ in $\psi_{\mathrm{P} 1}$ and $\psi_{\mathrm{P} 2}$ on the magnitude of the servicer's control inputs. 100

4.16 Experimental setup . . . . . . . . . . . . . . . . . . . 101

4.17a Phase one of experiment . . . . . . . . . . . . . 103

4.17bPhase two of experiment . . . . . . . . . . . . . . 103

4.18 Manipulator joint position error . . . . . . . . . . . . . . . . 104 
4.19 Manipulator joint velocity error . . . . . . . . . . . . . . . . . . . . . . . . 104

4.20 Measured end-effector's force/torque for Cases 1 and 2 in frame $\Sigma_{\mathrm{e}}$ located at the end-effector. Top plot presents torque about $\mathrm{z}$ axis of $\Sigma_{\mathrm{e}}$. Bottom two plots present force along $\mathrm{x}$ and $\mathrm{y}$ axis of $\Sigma_{\mathrm{e}} . \ldots \ldots$. . . . . . . . . . 105 


\section{Chapter 1}

\section{Introduction}

\subsection{Background}

The current state of space activity has been expanding significantly with increased access to space by developing nations. Space activates range from space exploration to commercial application: exploring the surface of Mars to ensuring effective telecommunication and weather forecasting on Earth. Its impact on our daily lives is hard to notice due to their common place in our society, but their significance on the functionality of our day to day activities is ever so important that if gone, it would have a negative impact on our livelihood.

The increased utilization of the space environment without global cooperation to address the growing population of space debris presents a significant problem for future access to space regardless of the purpose. The term space debris encompasses both man made and natural objects and is estimated to be composed of over 8700 objects larger than $10-30 \mathrm{~cm}$ in Low Earth Orbit (LEO) [40]. The composition of the debris field can range from paint flecks and satellite coolant droplets to spent rocket upper stages and decommissioned or malfunctioning satellites [4,40].

The state of the space debris field posses a threat to current orbiting satellites and have the potential to under go exponential growth through cascading collisions (Kessler effect [4, 14]). The high impact collision that occurred in 2009 between deactivated Kosmos 2251 and operating Iridium 33 satellite with a relative speed greater than $11 \mathrm{~km} / \mathrm{s}$ resulted in more debris and illustrates the risk space debris poses on current operational satellites [73]. In 2007, a militarized test of a direct-ascent anti-satellite (ASAT) weapon on a weather satellite, Fengun-1C, 
produced at least 2087 additional pieces of space debris reported by the US Space Surveillance Network (SSN) [31]. The growing debris field posses a direct threat to the operational safety of the International Space Station (ISS) as it carries out collision-avoidance manoeuvers if the chance of collision exceeds a certain tolerance [40]. As a result, NASA has developed a collision risk assessment process for it high-value robotic spacecraft to prevent loss by collision with space debris [43].

The first appearance of space debris mitigation was proposed by Lubo Perek in 1979, who's recommendations are still valid today: re-orbiting of GEO spacecraft into a disposal orbit at the end-of-life [27]. Since then, various international organizations have proposed space debris mitigation guidelines [65]. These guidelines impose design and operational considerations that would aim to minimize debris released during normal operations, as well as limit the long-term presence of a satellite in its orbital region after the completion of its mission. An example of this is the debris mitigation policy proposed and practiced by the Inter-Agency Space Debris Coordination Committee (IADC), an international technical body composed of various space agencies such NASA, the European Space Agency (ESA), and Japanese Space Agency (JAXA). However, these guidelines often fall on deaf ears as there is no requirement by all spacefaring nations on compliance with the guidelines for access to space [65,74]. In addition, the proposed guidelines, while an attempt to mitigate the addition of more debris, do not address the current state of the debris field.

Proposed solutions to address the current state of the space debris field vary from a reactive approach of physically removing space debris to a proactive approach of the capture and repair of malfunctioning satellites. Regarding the active removal of space debris, various strategies for the capture and removal of space debris have been proposed in the literature and range from the use of a net, harpoon, space tether-gripper or space manipulator(s) to capture and dispose of space debris [55] (Fig. 1.1). However, the capture and repair of malfunctioning satellites, also known as on-orbit servicing (OOS), not only addresses the problem of space debris mitigation, but it is also an attractive fiscal approach for companies and space agencies as it extends the operating life time of space assets [66].

Regardless of the proposed solution, the paradigm of space activity involves the use of robotic probes as they remove significant design considerations that are associated with a human in the loop. The role of robots in space consist of manoeuvring payloads, replacing/assembling components on the ISS to supporting astronauts during spacewalks. One solution for conducting OOS missions that is under investigation by various space agencies and 

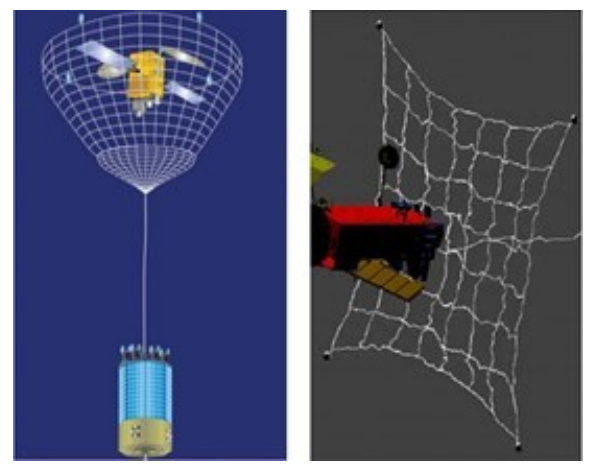

Figure 1.1: Example of space debris removal using net [13].

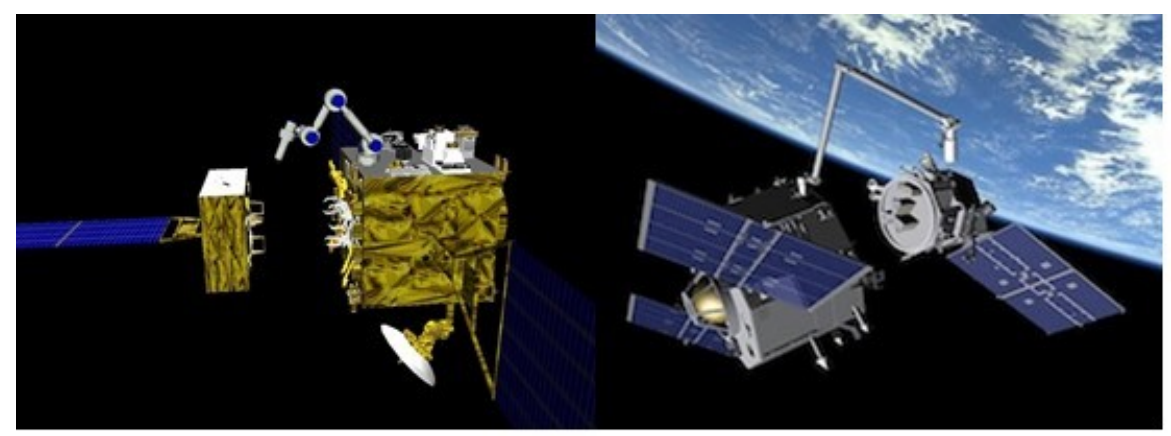

Figure 1.2: JAXA ETS-VII mission on the left [45]. DARPA Orbital Express Program on the right [23].

research groups is a robotic manipulator attached to a satellite base, and is denoted as the servicer [8,27]. The malfunctioning satellite to be serviced, is denoted as the target and is noncooperative and tumbling. The term non-cooperative implies that the target does not broadcast any information regarding its current state of motion, nor can it control any of its on board actuators to adjust its attitude in order to assist the servicing satellite in its capture. Furthermore, it also implies that the target was not designed to be grasped by the servicer, and hence, contains no dedicated grasping surface.

Several on-orbit experiments have been conducted by different space and government agencies to test and study the technology readiness of various designs and approaches for OOS (Fig. 1.2). These experiments have been conducted on cooperative targets. The Engineering Test Satellites VII (ETS-VII) was launched by JAXA in 1997 to test various robotics technologies and to demonstrate its applicability in OOS related task such as: autonomous rendezvous/docking, orbital replacement unit exchange and deployment, and dynamically coordi- 


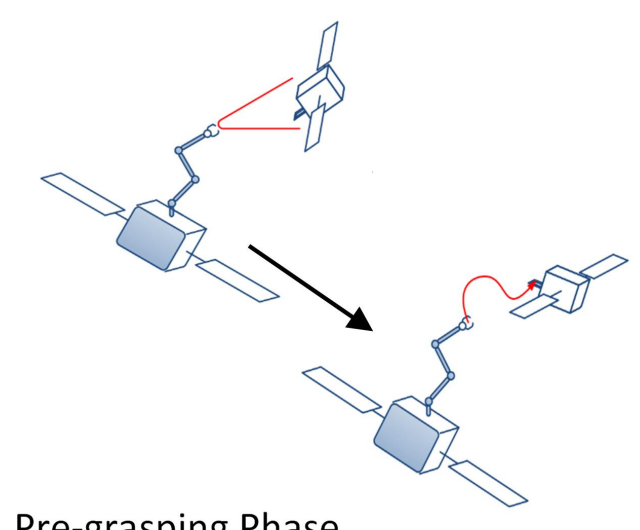

Pre-grasping Phase

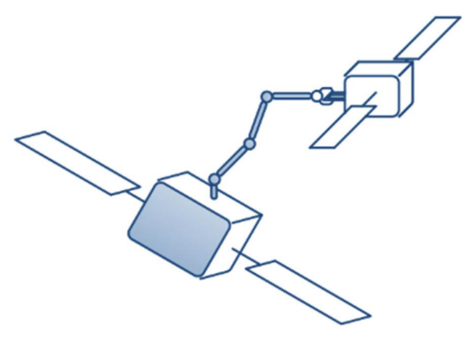

Grasping

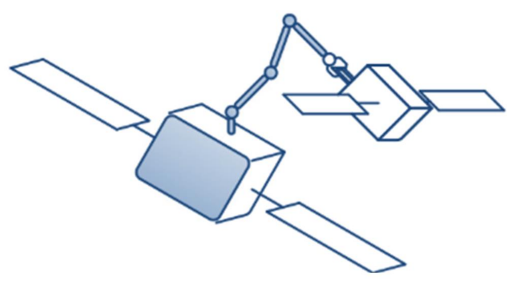

Post-grasping phase

Figure 1.3: Phases of satellite capture sequence [22].

nated control between the manipulator and servicer's base to name a few [70]. The Robotic Refueling Mission (RRM) is a joint project between NASA and Canadian Space Agency (CSA) with the aim of demonstrating how robots can service and refuel satellites in space. Additional efforts by NASA, the US Department of Defence, DARPA, and German Aerospace Center (DLR) have tested and proposed concepts for future experiments for the purpose of OOS demonstration [24, 37, 46].

In the literature, researchers have separated the capture process of a non-cooperative, tumbling target by a servicer into different phases due to the complexity of the problem: pregrasping phase, grasping and post-grasping phase [2,8] (Fig. 1.3). The pre-grasping phase is concerned with moving the manipulator from its initial position to a predetermined position to intercept the target's grasping fixture. In [8,19,75], a vision system along with a nonlinear state estimator are utilized to predict the motion of the non-cooperative target, and hence the grasping fixture on the target. Utilizing the predicted motion of the target, different strategies in the literature have been designed to intercept the target's grasping point with the aim of minimizing impact or subjected to physical constraints of the servicer's manipulator [8, 32, 35, 53].

In the grasping phase, the servicer is concerned with physically grasping the target and establishing a firm connection with the target. The grasping of a non-cooperative target is an active area of research and is a difficult problem to address due in part that orbiting satellites do not have dedicated grasping points. However, it has been proposed that potential grasping points are the Payload Attach Fitting or the nozzle cone of an apogee kick motor as these components have high structural strength that can be used to connect the target to the servicer [71]. 
The grasping of these points is still a challenging task due to the uncertainty in their position and velocity estimates at the moment of grasping. The uncertainty arises due to estimation error in the estimated position and velocity of the grasping point and may lead to unexpected contact between the servicer's end-effector and target's grasping surface. Active and passive compliance have been proposed to mitigate the effect of unexpected contact between the servicer and target in order to maintain contact with the target in the grasping phase [51, 59, 71]. Additional studies have been presented on how to minimize the attitude disturbance of the servicer's base due to unexpected contact with the target [42, 48].

Accommodating the position and velocity uncertainty of the servicer's grasping point can be addressed with the design of the grasping mechanism at the servicer's end-effector. Various grasping mechanisms have been proposed in the literature and are simple and robust to grasping point uncertainty [20,26]. However, interaction force/torque limits at which these mechanisms will fail must be taken into account in the post-grasping phase. In the post-grasping phase it is assumed that the servicer has grasped the target and is rigidly connected at the target's grasping point. A detumbling control strategy for the servicer must be formulated to bring the tumbling motion of the target to rest while taking into account changes in the servicer's inertial parameters due to the unknown target grasped at the servicer's end-effector. Furthermore, the detumbling control strategy must take into consideration interaction force/torque limits at the grasping location as this may result in damage of the servicer's grasping mechanism or target's grasping point and/or saturation of the servicer's attitude control system [7, 9, 47, 65, 66]. The focus of this dissertation is on the development of a detumbling control strategy for the postgrasping phase of the capture sequence.

\subsection{Review of Literature on Post-Grasping Phase}

The relevant literature pertaining to the post-grasping phase is reviewed in this section. First a review of existing post-grasping detumbling control strategies are presented under ideal conditions in which the target's inertial parameters (mass, inertia tensor, location of center of mass) are assumed known. After, a review of parameter identification techniques are presented, followed by a review of detumbling strategies that aim to address the problem of detumbling of a non-cooperative target with parameter uncertainty in the post-grasping phase. 


\subsubsection{Ideal Scenario}

Under the assumption that the target's inertial parameters are known, a detumbling strategy that takes into consideration the servicer's end-effector force/torque limits at the grasping location can be developed. In [6,7, 21], it was shown that applying a torque along the target's angular momentum would bring its tumbling motion to rest in minimal time. With knowledge of the target's inertial parameters and a known torque limit, a detumbling trajectory can be determined for the manipulator to track. This was made possible by subjecting the target's dynamics to a torque applied opposite to the its angular momentum vector. In [9], both the end-effector's force and torque limits are considered. The objective in this case was to bring the target's linear and angular momentum to zero. Stability analysis revealed that the detumbling torque should be applied along the target's angular momentum vector, while the applied force is along a unit vector that is computed as a function of the target's linear and angular momentum as well as its location of center of mass. In [35], an optimal joint damping trajectory to dampen the target's relative motion is presented. Similar to [6, 7, 9], the authors of [54,65] made use of the target's inertial parameters to plan a detumbling trajectory that would bring the target's tumbling motion to rest in minimum time while being subjected to a torque limit at the endeffector. In [6, 7, 9, 65], coordinated control of the servicer's base and its manipulator (i.e., [12,17]) is implemented to simultaneously track the desired end-effector detumbling trajectory and to reject the gained target's momentum as it is brought to rest.

In [72], utilization of the target's inertial parameters allowed the development of a control strategy that ensured a bias momentum distribution in the servicer prior to capture so as to eliminate base attitude disturbance after capture of the target. This approach would pre-load the servicer system, either its manipulator or reaction wheels or both, with momentum that would cancel that of the target's after capture. In [18], a distributed momentum control (DMC) is proposed to manage the angular momentum in the servicer-target system such that no base rotation occurs. The authors also made use of reaction null space control (RNSC) to manage the angular momentum distribution in the servicer-target system while performing joint damping in the null space of the primary momentum management task.

The above mentioned detumbling strategies for the post-grasping phase were developed under the assumption that the target's inertial parameters were accurately known, and further studies were not conducted on their performance subjected to uncertainties in the target's inertial parameters. However, it is unrealistic to assume that these parameters are known prior to 
detumbling (e.g., no practical way to measure remaining propellant in a malfunctioning satellite). Furthermore, it has been proposed that the target's inertial parameters can be identified with the use of parameter identification techniques prior to the post-grasping phase so that detumbling strategies that require their use can be implemented.

\subsubsection{Identifying the Target's Inertial Parameters}

Detumbling strategies discussed in the prior section required knowledge of the target's inertial parameters in the formulation of their detumbling controller for the post-grasping phase. As mentioned, it is unrealistic to assume that these parameters are known prior to post-grasping phase. However, it has been proposed that the target's inertial parameters can be identified prior to the post-grasping phase. As a result, in the literature, various techniques have been proposed to facilitate parameter identification and can be classified into three different categories: vision base [5, 8, 10], momentum and force based [37, 41].

Vision based techniques cannot identify the mass of the target but can estimate ratios of the target's moment of inertia in the pre-grasping phase [5,8, 10]. From these dimensionless ratios, it is not possible to identify the actual inertial values to be used in the post-grasping phase. Vision based techniques are ideally utilized in the pre-grasping phase to predict the motion of the target's grasping point for the purpose of capturing the target.

Momentum and force base techniques in [37, 41] can be utilized once the target has been captured, and are based on the equation of motion of the system expressed at the velocity and acceleration level, respectively. Both techniques can identify the inertial parameters of the target, however, convergence is dependent on the excitation of the input motion of the combined servicer-target system. In [37], different manipulator motions were utilized to demonstrate the effect of persistent excitation on parameter identification utilizing the force and momentum base techniques.

In [44], the authors introduced a new phase to the capture sequence that would transition from the grasping phase to the post-grasping phase. In the new phase, the authors proposed an adaptive reactionless control algorithm to generate reactionless manipulator motion with an unknown target attached to the end-effector. The term reactionless implies motion of the manipulator arm that would not induce a reaction motion on to the base, thus leaving its attitude undisturbed. Simultaneously, the authors utilized the manipulator's joint rates along with the servicer's base linear and angular velocities as measurement inputs to a momentum based algo- 
rithm in order to identify the target's inertial parameters. However, convergence of the target's inertial parameters cannot be guaranteed as it is directly related to the level of excitation of the input (motion of the combined servicer-target system) to the parameter identification scheme.

The dependence on parameter identification techniques to determine the target's inertial parameter as a prior for the post-grasping phase will not lead to the successful detumbling of the target. Convergence of the estimated target's inertial parameters is possible if the motion of the combined system (servicer and target) is sufficiently exciting to identify the target's inertial parameters. This cannot be guaranteed prior to the post-grasping phase and hence, cannot guarantee convergence of estimates of the target's inertial parameters to their true value. Detumbling strategies in the post-grasping phase cannot assume that the target's inertial parameters are known a prior and must be design to account for the unknown target's inertial parameters.

\subsubsection{Post-grasping Phase with Unknown Target}

As it is unrealistic to assume that the target's inertial parameters are known prior to the postgrasping phase, there are a number of control strategies proposed in the literature that achieve detumbling without perfect knowledge of the target's inertial parameters.

Addressing target parameter uncertainty, the authors of [47] and [66] have proposed the delineation of a detumbling trajectory without requiring accurate knowledge of the target's inertial parameters and subjected to end-effector's force/torque or torque limits, respectively. In order to respect end-effector force/torque limits, the detumbling trajectory in [47] is modified with the use of force/torque measurements at the end-effector. The detumbling trajectory in [66] was formulated only considering end-effector torque limit and assumed that the location of the target's center of mass is known. Coordination control of the servicer's satellite base and manipulator's end-effector to track the desired detumbling trajectory with an uncertain target attached to it was not addressed in [47]. In [66] and similar to [3, 60], impedance control is implemented with the use of end-effector's force/torque measurements to track the desired detumbling trajectory with an uncertain target attached to the servicer's end-effector. The implementation of these detumbling strategies required force/torque measurements at the servicer's end-effector. This can be a difficult requirement to satisfy as obtaining force/torque measurements in space can be challenging due to the harsh environment of space [38].

Robust coordination control strategies of the servicer's satellite base and manipulator with- 
out the need of force/torque measurements at the end-effector were proposed in [29, 74, 76]. In [74], an adaptive sliding mode controller is proposed to track a reference trajectory in the post-grasping phase in order to detumble a non-cooperative target with uncertain inertial parameters. The authors achieve robustness to target parameter uncertainty without the need of end-effector's force/torque measurements, but their proposed control strategy requires measurements of the servicer's manipulator joint acceleration and base angular acceleration.

The authors of [76] proposed an adaptive sliding mode disturbance observer to estimate the disturbance created by the unknown target in order to compensate for it while tracking a desired joint space trajectory and regulating the servicer's base attitude. In [29], an adaptive variable structure control method was applied to implement a robust coordination controller between the servicer's satellite base and manipulator that addressed parametric uncertainties in the servicer's system. In [29,76], the authors do not consider the detumbling problem in the post-grasping phase. However, the approach can be utilized to compensate for parametric uncertainties associated with an unknown target attached to the servicer's end-effector while attempting to track a desired detumbling trajectory. Furthermore, while the above-mentioned robust coordination control strategies of the servicer address parametric uncertainty associated with the unknown target ( [29,74,76]), they do not take into consideration limits on the servicer's control inputs. In practical application, the servicer will be subjected to magnitude limits on its control inputs because of physical limits of the onboard actuators. A controller design for coordination between the servicer's base and manipulator must consider limits on the control input as they may lead to instability if not considered or poor performance of the servicer in the detumbling task.

\subsection{Research Focus and Contributions}

The research problem to be addressed can be summarized as follows:

To develop a detumbling control strategy to detumble a non-cooperative tumbling target with unknown inertial parameters, using a space robot that consists of a robotic manipulator attached to a satellite while being subjected to force/torque limits at the grasping location.

From the overview of the previous section it should be clear that the formulation of a detumbling control strategy must respect end-effector force/torque limits of the space robot without prior knowledge of the target's inertial parameters (mass, inertia tensor, location of 
center of mass). Prior studies have formulated detumbling strategies with the assumption of accurate knowledge of the target's inertial parameters. However, obtaining accurate estimates of the target's inertial parameters is difficult, and parameter uncertainty may lead to instability and violation of the end-effector's force/torque limits if not taken into account in the detumbling controller's design. Force/torque limits are an important consideration in post-grasping phase. Force/torque limits can be determined based on the structural limitation of the grasping mechanism at which point it will fail. Furthermore, force/torque limits at the servicer's end-effector are based on the rate at which momentum is being transferred from the target to the servicer. This rate is specified such that the servicer's control inputs can reject the gained momentum from the target in order to avoid tumbling of the combined servicer-target system in the post-grasping phase.

This dissertation presents two detumbling control strategies based on the availability of force/torque measurements at the servicer's end-effector. Obtaining force/torque measurements at the end-effector adds additional complexity as the design and housing of the sensor must be taken into account for successful operation in the harsh space environment. The two presented detumbling strategies will be compared at the end of this dissertation.

If force/torque measurements at the end-effector are available, detumbling of the target is achieved with the use of force control. This allows the formulation of a detumbling strategy that enables compliance with the unknowns target's tumbling motion as damping is applied to bring its tumbling motion to rest. Detumbling of the target is achieved by controlling the space robot to apply a reference force/torque to the target. The reference force/torque is designed to detumble the target while respecting force/torque limits at the servicer's end-effector, without the use of the target's inertial parameters. The reference force/torque is computed utilizing only known parameters such as end-effector's force/torque limits and end-effector's linear and angular velocity.

The implementation of this detumbling strategy is presented with and without compensation for changes in the servicer's inertial parameters due to the grasped target. In the case of no compensation for changes in the servicer's inertial parameters, the servicer is controlled to apply the reference force/torque to the target. A detumbling criteria is then formulated in the form of bounds on the target's inertial parameters and tumbling rate for which this approach can detumble (domain of attraction). Evaluation of the detumbling criteria can be done utilizing estimates of bounds on the target's inertial parameters. The availability of bounds on the target's inertial parameters is a realistic assumption as these bounds can be determined based on 
pre-launch data of the malfunctioning satellite slated to be serviced. The resultant detumbling controller is simple, however, if the detumbling criteria cannot be satisfied a robust compensator is presented to ensure stable detumbling of the target and is designed based on bounds of the target's unknown inertial parameters. Furthermore, bounds on the target's unknown inertial parameters are estimated in real-time in order to reduce the robust control gains.

Alternatively, if force/torque measurements at the end-effector are not available, a tracking control detumbling strategy is presented. A robust coordination controller is developed to control the servicer's base satellite and its manipulator to track a desired detumbling trajectory with the unknown target attached to the end-effector while rejecting the target's gained momentum. The desired detumbling trajectory is delineated using only bounds on the target's inertial parameters and subjected to end-effector force/torque limits. The proposed robust coordination controller takes into account magnitude constraints on the servicer's manipulator and base attitude control inputs in the controller's design. This is accomplished by making use of the hyperbolic tangent function to model the magnitude constraints of the servicer's control inputs, which results in a system that is non-affine in its control inputs. An augmented model of the servicer is formulated to allow the development of the detumbling controller. Using bounds on the target's inertial parameters, robust adaptive control approach is utilized to design the detumbling controller with the backstepping technique to ensure successful detumbling of the unknown target attached to the end-effector.

The main contributions from both detumbling strategies are as follows:

1. Prior detumbling strategies in the literature required accurate knowledge of the target's inertial parameters in the formulation of their detumbling strategies when taking into account interaction force/torque limits at the servcier's end-effector. Studies that take into account force/torque limits at the servicer's end-effector without accurate knowledge of the target's inertial parameters make simplifying assumptions on the target's inertial parameters (i.e., assume target's location of center of mass is known) or do not consider both force and torque limit at the servicer's end-effector. Furthermore, detumbling strategies that achieve detumbling with the unknown target attached to the servicer's end-effector do not consider force/torque limits at the servicer's end-effector. Both detumbling strategies presented in this study achieves detumbling of the target subjected to force and torque limits at the servicer's end-effector without prior accurate knowledge of the target's inertial parameters. 
2. The reference detumbling force/torque in the force control based detumbling strategy is uniquely defined such that it does not require knowledge of the target's inertial parameters in its formulation and takes into account force/torque limits at the servicer's endeffector. This is unlike prior approaches in the literature that required knowledge of the target's inertial parameters in the formulation of their reference detumbling force/torque.

3. In the tracking control based detumbling strategy, a procedure is presented to delineate the desired detumbling trajectory. The procedure delineates a desired detumbling trajectory subjected to force and torque limits at the end-effector without requiring accurate knowledge of the target's inertial parameters.

4. In the tracking control based detumbling strategy, coordination control of the servicer's base and manipulator is developed to track the desired end-effector detumbling trajectory and to reject the target's gained momentum. The coordination controller of the servicer's end-effector and base takes into account magnitude limits on the servicer's control inputs and do not require force/torque measurements at the servicer's end-effector.

5. The presented force control and tracking control based detumbling strategies both contain a criteria that can be used to determine if detumbling a target can be accomplished based on the servicer's design. The servicer's design will dictate the maximum force/torque it can experience at its end-effector such that its control inputs can reject the target's momentum without causing the servicer-target system to tumble in the post-grasping phase. Evaluation of each criteria is carried out using bounds on the target's inertial parameters, the target's tumbling rate and the servicer's force and torque limit.

\subsection{Organization of Dissertation}

This dissertation consists of five chapters and is organized as follows. A detailed literature review and background information is given in the first chapter. This chapter also highlights the research objective and contributions obtained from this research. Chapter 2 presents the mathematical notation and assumptions utilized throughout this dissertation. The assumptions presented in this chapter define the conditions for the post-grasping phase. The dynamics and kinematics of the servicer and target are presented in this chapter as well. 
Chapter 3 presents the formulation of the detumbling strategy under the condition that force/torque measurements at the end-effector are available. The realization of this strategy is first presented in this chapter by defining the desired force and torque to be applied to the unknown target so that its tumbling motion goes to zero and to allow compliance with its residual motion. In the first half of Chapter 3, this approach is presented with no compensation for changes in the servicer's inertial parameters due to the grasped unknown target. The detumbling criteria is presented in the form of bounds on the target's inertial parameters and tumbling rate for which the approach can detumble (domain of attraction). Numerical simulation study of the detumbling strategy is conducted and the results are reported.

The second half of Chapter 3 presents a robust adaptive controller to compensate for changes in the servicer's inertial parameters due to the grasped target. This is accomplished in two steps. First a bounding function is derived to robustly compensate for the addition of the unknown target grasped at the servicer's end-effector in order to achieve stable detumbling of the target. The bounding function is computed using bounds on the target's inertial parameters. Following this, an adaptive technique is utilized to allow estimation of bounds on the target's inertial parameters in real-time. This eliminates the use of conservative estimates of bounds on the target's inertial parameters in the bounding function. Numerical simulation study of the detumbling strategy is conducted and the results are reported.

Chapter 4 presents the formulation of the detumbling strategy under the condition that force/torque measurements at the end-effector are not available. The approach presented in this chapter achieves detumbling of the target by tracking a desired detumbling trajectory. The delineation of the desired detumbling trajectory subjected to end-effector force/torque limit is presented. The formulation of the controller to track the desired detumbling trajectory and reject the gained momentum from the target is presented. Magnitude limits on the servicer's control inputs are taken into account in the controller's design. An upper bound on the endeffector force/torque experienced in the post-grasping phase is derived and presented. $\mathrm{Nu}$ merical simulation study is carried out using a 7-degree-of-freedom manipulator attached to a satellite base to detumble a target using the presented approach and the results are reported. Furthermore, an experiment is conducted to evaluate the design procedure used to delineate the desired detumbling trajectory and the results are reported.

In Chapter 5, a summary and discussion of the advantages and disadvantages of the two detumbling control strategies are presented. Following this, concluding remarks are given and the direction of future work is presented. 


\section{Chapter 2}

\section{Problem Formulation}

This Chapter presents the assumptions used in formulating the two proposed detumbling strategies in this dissertation. The mathematical notation utilized throughout this dissertation is presented as well as the servicer's and target's kinematics and dynamics. The Chapter concludes with a description of the control problem for the post-grasping phase.

\subsection{Post-grasping Phase}

Prior to the commencement of on-orbit servicing of the tumbling malfunctioning target satellite, it is required that the target must be captured. As mentioned in the previous Chapter, the research community has partitioned the capture process of the target into three different phases: pre-grasping phase, grasping phase and post-grasping phase. These different phases were presented by the research community to facilitate research into different problems that occur throughout of the capture process. These challenges arise primary as the target's inertial parameters are not known, the target is tumbling and is non-cooperative.

A malfunctioning satellite will usually not have a functioning attitude control system and it is common for them to tumble as the angular momentum stored in their attitude control system will start migrating to the body of the satellite. The inertial properties of the malfunctioning satellite such as its mass, inertia tensor and location of center of mass from the grasping point are usually uncertain because there is no practical way to measure remaining propellant fuel in zero gravity [8].

The pre-grasping and grasping phases are responsible to for ensuring that the servicer's end- 
effector can intercept the target grasping point and physically grasp the target. This must be accomplished in light of the above difficulties associated with a malfunctioning target satellite. In the post-grasping phase, the servicer is responsible for bringing the tumbling motion of the target to rest subjected to interaction force/torque limits at the grasping location while rejecting the target's gained momentum in order to avoid tumbling of the combined servicertarget system.

The following assumptions are utilized in developing the proposed detumbling strategy for the post-grasping phase:

1. the base's linear and angular velocities are measurable;

2. the target is rigidly attached to the end-effector after grasping (firmly grasped); and

3. the relative linear velocity between the center of mass of the servicer and target prior to capture is zero.

The first assumption implies that measurements of the servicer system are available from the inertial frame. This can be made possible if there exists an inertial observer such as a camera on an external space structure as proposed by [49], or a second satellite in formation with the servicer. To realize the last assumption, vehicular operations of the servicer satellite are assumed to have been utilized to guide the servicer to a pose where the relative linear velocity of the servicer and target are near zero and the servicer's manipulator can reach out and capture the target. Under these conditions, the combined servicer-target system after capture will have zero linear momentum relative to the inertial observer as the problem of absorbing and dissipating the gained linear momentum by the servicer with the use of external jet thruster is not addressed. These assumptions are common in the literature on detumbling of a noncooperative target [18, 44, 47, 72].

In addition to the above assumptions that defined the post-grasping phase, it is assumed that the detumbling process occurs sufficiently slow such that flexibility in the manipulator joints and links and the servicer's base can be neglected [61]. Thus, the servicer is assumed to consists of multiple rigid bodies. The mass of each individual body are time invariant and their respective location of center of mass within each body is fixed. The same assumption is made for the malfunctioning non-cooperative target. This can be rationalized of the motion of targets can be characterized as a slow rotation about a major axis [53]. For example, ground based observation of ADEOS 1 described its attitude motion as composed of two rotational 
components: $0.1 \mathrm{deg} / \mathrm{s}$ about the satellite's main body and another about the satellite's boom of about $0.4 \mathrm{deg} / \mathrm{s}$ [77]. Observation of Envisat resulted in an average rotation rate of 3.5deg/s [62].

As a result of the above stated assumptions, the following Corollary can be stated:

Corollary 1. The servicer is composed of multiple rigid bodies that are physically constrained to each other via revolute joints and the target is rigidly attached to the servicer's end-effector (Assumption 2), i.e., the maximum distance between the end-effector and the center of mass of any rigid body of the servicer/target system is geometrically constrained. From Assumption 3, it follows that the end-effector's position is also bounded from an inertial frame of reference located at the center of mass of the combined servicer/target system.

\subsection{Mathematical Notation}

The following notations are used:

- The left superscript, ${ }^{(\cdot)}\left(_{-}\right)$, represents the frame of reference the vector $\left(_{-}\right)$is resolved in. This notation is dropped for vectors expressed in the inertial frame.

- A right upper superscript $\mathrm{d}$ as in $(\cdot)^{\mathrm{d}}$, denotes the desired value of $(\cdot)$.

- The rotation matrix that transforms vector from reference frame A to reference frame B is denoted as follows: ${ }^{\mathrm{B}} \mathbf{R}_{\mathrm{A}} \in \mathbb{R}^{3 \times 3}$.

- $\mathbf{E}_{n} \in \mathbb{R}^{n \times n}$ is an identity matrix.

- $\lambda_{\max }(\cdot)$ and $\lambda_{\min }(\cdot)$ denote the maximum and minimum eigenvalues of matrix $(\cdot)$, respectively.

- For the vector $\mathbf{x}=\left[\begin{array}{lll}\{\mathbf{x}\}_{1} & \{\mathbf{x}\}_{2} & \{\mathbf{x}\}_{3}\end{array}\right]^{\mathrm{T}} \in \mathbb{R}^{3}$, the skew-symmetric matrix is denoted as $\mathbf{x}^{\times}$such that $\mathbf{x}^{\times} \mathbf{a}=\mathbf{x} \times \mathbf{a}$ for any $\mathbf{a} \in \mathbb{R}^{3}$, where the skew-symmetric matrix of $\mathbf{x}$ is defined as follows:

$$
\mathbf{x}^{\times}=\left[\begin{array}{ccc}
0 & -\{\mathbf{x}\}_{3} & \{\mathbf{x}\}_{2} \\
\{\mathbf{x}\}_{3} & 0 & -\{\mathbf{x}\}_{1} \\
-\{\mathbf{x}\}_{2} & \{\mathbf{x}\}_{1} & 0
\end{array}\right]
$$

- $(\cdot)^{+}$denotes the Moore-Penrose pseudoinverse of the matrix $(\cdot)$. 


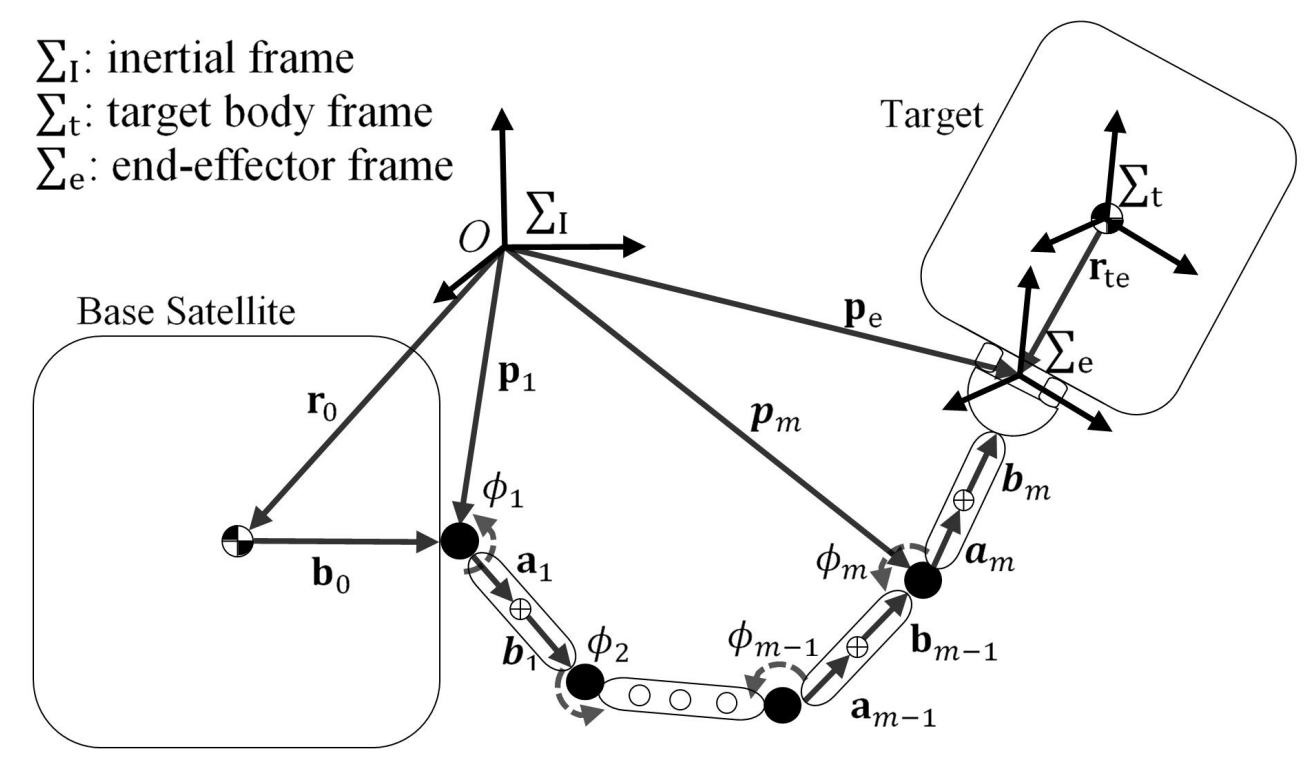

Figure 2.1: Space manipulator and base (servicer) with target

\subsection{Kinematics of Servicer}

The basic kinematic equation of a $m$ degree of freedom (DOF) manipulator mounted to a satellite base is presented in this Section. As per Fig. 2.1, the system is composed of the manipulator and satellite base and is denoted as the servicer. It consists of $m+1$ rigid bodies. The formulation presented in this section does not consider the target. However, as the target is assumed rigidly attached to the end-effector it is possible to consider it as an extension of the last link.

From Fig. 2.1, let $\mathbf{a}_{i}$ denote the vector pointing from joint $i$ to the mass center of link $i$ and let $\mathbf{b}_{i}$ denote the vector from the mass center of link $i$ to joint $i+1$. The satellite base is regarded as link $i=0$. This formulation is comparable for the servicer's base by noting that $\mathbf{r}_{0}$ denotes the vector from the servicer's base center of mass relative to the inertial frame, $\Sigma_{\mathrm{I}}$, at point $O$ in Fig. 2.1. Furthermore, $\mathbf{b}_{0}$ denotes the vector from the mass center of servicer's base to joint $i=1$. Note that all these vectors are expressed in the inertial frame. From Fig. 2.1, it becomes trivial to express the position of the end-effector as:

$$
\mathbf{p}_{\mathrm{e}}=\mathbf{r}_{0}+\mathbf{b}_{0}+\sum_{i=1}^{m} \mathbf{l}_{i}
$$

where $\mathbf{l}_{i}=\mathbf{a}_{i}+\mathbf{b}_{i}$ 
The end-effector's linear velocity can be obtained by differenciating both sides of 2.2 with respect to time and results in the following:

$$
\mathbf{v}_{\mathrm{e}}=\mathbf{v}_{\mathrm{b}}+\mathbf{w}_{\mathrm{b}} \times \mathbf{b}_{0}+\sum_{i=1}^{m} \mathbf{w}_{i} \times \mathbf{l}_{i}
$$

where $\mathbf{v}_{\mathrm{e}} \in \mathbb{R}^{3}$ denotes the end-effector's linear velocity, $\mathbf{v}_{\mathrm{b}} \in \mathbb{R}^{3}$ and $\mathbf{w}_{\mathrm{b}} \in \mathbb{R}^{3}$ denotes the servicer's base center of mass linear and angular velocity, respectively. $\mathbf{w}_{i}$ denotes the angular velocity of link $i$ and can be expressed as:

$$
\mathbf{w}_{i}=\mathbf{w}_{\mathrm{b}}+\sum_{j=1}^{i} \mathbf{k}_{j} \dot{\phi}_{j}
$$

where $\mathbf{k}_{j} \in \mathbb{R}^{3}$ is the unit vector indicating the rotation axis of joint $j$ and $\dot{\phi}_{j}$ denotes the joint rate of joint $j$.

Making use of (2.4) and 2.2) in 2.3 results in the following:

$$
\mathbf{v}_{\mathrm{e}}=\mathbf{v}_{\mathrm{b}}+\mathbf{w}_{\mathrm{b}} \times\left(\mathbf{p}_{\mathrm{e}}-\mathbf{r}_{0}\right)+\sum_{i=1}^{m}\left\{\mathbf{k}_{i} \times\left(\mathbf{p}_{\mathrm{e}}-\mathbf{p}_{i}\right)\right\} \dot{\boldsymbol{\phi}}_{i}
$$

where $\mathbf{p}_{i}$ denotes the position of joint $i$. From 2.4 the end-effector's angular velocity can be expressed as:

$$
\mathbf{w}_{\mathrm{e}}=\mathbf{w}_{\mathrm{b}}+\sum_{i=1}^{m} \mathbf{k}_{i} \dot{\phi}_{i} .
$$

Making use of 2.5) and (2.6), the end-effector's linear and angular velocity can be expressed in a more compact form that will be utilized in the formulation of the servicer's equation of motion:

$$
\left[\begin{array}{c}
\mathbf{v}_{\mathrm{e}} \\
\mathbf{w}_{\mathrm{e}}
\end{array}\right]=\mathbf{J}_{\mathrm{b}}\left[\begin{array}{c}
\mathbf{v}_{\mathrm{b}} \\
\mathbf{w}_{\mathrm{b}}
\end{array}\right]+\mathbf{J}_{\mathrm{m}} \dot{\phi}_{\mathrm{m}}
$$

where the two Jacobian matrices are introduced and the manipulator joint rates are combined into a joint vector as follows:

$$
\dot{\phi}_{\mathrm{m}}=\left[\begin{array}{llll}
\dot{\phi}_{1} & \dot{\phi}_{2} & \ldots & \dot{\phi}_{m}
\end{array}\right]^{\mathrm{T}} \in \mathbb{R}^{m}
$$




$$
\begin{aligned}
& \mathbf{J}_{\mathrm{b}}=\left[\begin{array}{cc}
\mathbf{E}_{3} & -\mathbf{p}_{0 \mathrm{e}}^{\times} \\
0 & \mathbf{E}_{3}
\end{array}\right] \in \mathbb{R}^{6 \times 6}, \quad \mathbf{p}_{0 \mathrm{e}}=\mathbf{p}_{\mathrm{e}}-\mathbf{r}_{0} \\
& \mathbf{J}_{\mathrm{m}}=\left[\begin{array}{cccc}
\mathbf{k}_{1} \times\left(\mathbf{p}_{\mathrm{e}}-\mathbf{p}_{1}\right) & \mathbf{k}_{2} \times\left(\mathbf{p}_{\mathrm{e}}-\mathbf{p}_{2}\right) & \ldots & \mathbf{k}_{m} \times\left(\mathbf{p}_{\mathrm{e}}-\mathbf{p}_{m}\right) \\
\mathbf{k}_{1} & \mathbf{k}_{2} & \ldots & \mathbf{k}_{m}
\end{array}\right] \in \mathbb{R}^{6 \times m}
\end{aligned}
$$

The coefficient matrix of the base velocity term is denoted as the Jacobian matrix for the base and the coefficient matrix for the manipulator joint rates is the Jacobaina for ground-base manipulators. The end-effector velocity for the servicer is observed to be a linear combination of the manipulator joint rates and of the servicer's base velocities. The main difference between the kinematics of a space manipulator and that of a ground-base manipulator is the addition of the servicer's base velocity components in the end-effector's velocity. This represents the motion of ground-base manipulator base joint.

\subsection{Servicer's Momentum Equations}

In this Section, the momentum equations for the servicer will be presented. A compact form of these expression will be presented that expresses the total momentum for the servicer with respect to the location of the inertial frame at point $O$ in Fig. 2.1 and about the center of mass of the servicer's base. Let $\mathbf{P} \in \mathbb{R}^{3}$ and $\mathbf{L} \in \mathbb{R}^{3}$ represent the total linear and angular momentum, respectively, of the servicer with respect to the inertial frame. By definition they are defined as follows:

$$
\begin{gathered}
\mathbf{P}=\sum_{i=0}^{m} m_{i} \mathbf{v}_{i} \\
\mathbf{L}=\sum_{i=0}^{m}\left(\mathbf{I}_{i} \mathbf{w}_{i}+\mathbf{r}_{i} \times m_{i} \mathbf{v}_{i}\right)
\end{gathered}
$$

where $\mathbf{r}_{i} \in \mathbb{R}^{3}$ is the position vector of the center of mass of link $i, \mathbf{I}_{i} \in \mathbb{R}^{3 \times 3}$ is the inertia tensor of link $i$ with respect to its center of mass, $m_{i}$ is the mass of the rigid body link $i, \mathbf{w}_{i}$ is the angular velocity of link $i$ as defined in 2.4 and $\mathbf{v}_{i} \in \mathbb{R}^{3}$ is the linear velocity of center of mass of link $i$. Note that link $i=0$ denotes the servicer's base where its linear and angular velocities are denoted as $\mathbf{v}_{0}=\mathbf{v}_{\mathrm{b}}$ and $\mathbf{w}_{0}=\mathbf{w}_{\mathrm{b}}$. Furthermore, recall that all vectors are expressed in the inertial frame. 
From (2.5), changing the point of interest from the servicer's end-effector to the center of mass of link $i$ in (2.5) presents an equation for $\mathbf{v}_{i}$ of link $i$ as follows:

$$
\mathbf{v}_{i}=\mathbf{v}_{\mathrm{b}}+\mathbf{w}_{\mathrm{b}} \times\left(\mathbf{r}_{\mathrm{i}}-\mathbf{r}_{0}\right)+\sum_{j=1}^{i}\left\{\mathbf{k}_{j} \times\left(\mathbf{r}_{\mathrm{i}}-\mathbf{p}_{j}\right)\right\} \dot{\boldsymbol{\phi}}_{j}
$$

Replacing $\mathbf{v}_{i}$ in 2.9a) with 2.10 results in the following:

$$
\mathbf{P}=m_{\mathrm{total}} \mathbf{v}_{\mathrm{b}}+\mathbf{w}_{\mathrm{b}} \times m_{\mathrm{total}} \mathbf{r}_{0 \mathrm{~g}}+\mathbf{J}_{\mathrm{T} w} \dot{\phi}_{\mathrm{m}}
$$

where

$$
\begin{gathered}
m_{\mathrm{total}}=\sum_{i=0}^{m} m_{i} \\
\mathbf{r}_{0 \mathrm{~g}}=\mathbf{r}_{\mathrm{g}}-\mathbf{r}_{0} \\
\mathbf{r}_{\mathrm{g}}=\frac{\sum_{i=0}^{m} m_{i} \mathbf{r}_{i}}{m_{\mathrm{total}}} \\
\mathbf{J}_{\mathrm{T} w}=\sum_{i=1}^{m} m_{i} \mathbf{J}_{\mathrm{T} i}
\end{gathered}
$$

From 2.12a), $m_{\text {total }}$ denotes the total mass of the servicer and $\mathbf{r}_{\mathrm{g}}$ in $2.12 \mathrm{c}$ denotes the position of mass center of the whole system.

Similar substitution can be made for the servicer's total angular momentum. Replacing $\mathbf{w}_{i}$ with (2.4) and $\mathbf{v}_{i}$ with 2.10 in the servicer's total angular momentum in 2.9b) results in the following:

$$
\begin{aligned}
\mathbf{L} & =\sum_{i=0}^{m}\left[\mathbf{I}_{i}\left(\mathbf{w}_{\mathrm{b}}+\mathbf{J}_{\mathrm{R} i} \dot{\phi}_{\mathrm{m}}\right)+\mathbf{r}_{i} \times m_{i}\left(\mathbf{v}_{\mathrm{b}}+\mathbf{w}_{\mathrm{b}} \times\left(\mathbf{r}_{i}-\mathbf{r}_{0}\right)\right)\right] \\
& =\sum_{i=0}^{m} \mathbf{I}_{i} \mathbf{w}_{\mathrm{b}}+\sum_{i=1}^{m} \mathbf{I}_{i} \mathbf{J}_{\mathrm{R} i} \dot{\phi}_{\mathrm{m}}+\sum_{i=0}^{m} m_{i} \mathbf{r}_{i} \times \mathbf{v}_{\mathrm{b}}-\sum_{i=1}^{m} m_{i} \mathbf{r}_{i} \times\left[\left(\mathbf{r}_{i}-\mathbf{r}_{0}\right) \times \mathbf{w}_{\mathrm{b}}\right] \\
& +\sum_{i=1}^{m} m_{i} \mathbf{r}_{i} \times \mathbf{J}_{\mathrm{T} i} \dot{\phi}_{\mathrm{m}}
\end{aligned}
$$


where

$$
\mathbf{J}_{\mathrm{R} i}=\left[\begin{array}{lllllll}
\mathbf{k}_{1} & \mathbf{k}_{2} & \ldots & \mathbf{k}_{i} & 0 & \ldots & 0 \in \mathbb{R}^{3 \times m}
\end{array}\right] .
$$

Furthermore, the reduction in (2.13) resulted by noting that for $i=0, \mathbf{J}_{\mathrm{R} 0}=0, \mathbf{J}_{\mathrm{T} 0}=0$ and $\mathbf{r}_{0}-\mathbf{r}_{0}=0$.

The total momentum of the servicer can be expressed about the center of mass of the base. Let $\mathbf{P}_{\mathrm{b}} \in \mathbb{R}^{3}$ and $\mathbf{L}_{\mathrm{b}} \in \mathbb{R}^{3}$ denote the momentum of the servicer about center of mass of the base. The relationship between $\mathbf{P}, \mathbf{L}, \mathbf{P}_{\mathrm{b}}$ and $\mathbf{L}_{\mathrm{b}}$ is as follows:

$$
\begin{gathered}
\mathbf{P}_{\mathrm{b}}=\mathbf{P} \\
\mathbf{L}_{\mathrm{b}}=\mathbf{L}-\mathbf{r}_{0} \times \mathbf{P} .
\end{gathered}
$$

With the use of (2.10) and (2.11), the product $\mathbf{r}_{0} \times \mathbf{P}$ can be evaluated as follows:

$$
\mathbf{r}_{0} \times \mathbf{P}=m_{\text {total }} \mathbf{r}_{0} \times \mathbf{v}_{\mathrm{b}}-\sum_{i=1}^{m} m_{i} \mathbf{r}_{0} \times\left[\left(\mathbf{r}_{i}-\mathbf{r}_{0}\right) \times \mathbf{w}_{\mathbf{b}}\right]+\sum_{i=1}^{m} m_{i} \mathbf{r}_{0} \times \mathbf{J}_{\mathrm{T} i} \dot{\phi}_{\mathrm{m}}
$$

Substituting (2.16) and (2.13) into 2.15b) results in:

$$
\mathbf{L}_{\mathrm{b}}=m_{\text {total }} \mathbf{r}_{0 \mathrm{~g}}^{\times} \mathbf{v}_{\mathrm{b}}+\left[\sum_{i=1}^{m}\left(\mathbf{I}_{i}+m_{i} \mathbf{r}_{0 i}^{\times \mathrm{T}} \mathbf{r}_{0 i}^{\times}\right)+\mathbf{I}_{0}\right] \mathbf{w}_{\mathrm{b}}+\left[\sum_{i=1}^{m}\left(\mathbf{I}_{i} \mathbf{J}_{\mathrm{R} i}+m_{i} \mathbf{r}_{0 i}^{\times} \mathbf{J}_{\mathrm{T} i}\right)\right] \dot{\phi}_{\mathrm{m}}
$$

where $\mathbf{r}_{0 i}=\mathbf{r}_{i}-\mathbf{r}_{0}$.

Combining (2.11) and (2.17) allows for the compact representation of the servicer's momentum as:

$$
\left[\begin{array}{c}
\mathbf{P}_{\mathrm{b}} \\
\mathbf{L}_{\mathrm{b}}
\end{array}\right]=\left[\begin{array}{cc}
m_{\text {total }} \mathbf{E}_{3} & m_{\text {total }} \mathbf{r}_{0 \mathrm{~g}}^{\times \mathrm{T}} \\
m_{\text {total }} \mathbf{r}_{0 \mathrm{~g}}^{\times} & \mathbf{H}_{\mathrm{w}}
\end{array}\right]\left[\begin{array}{c}
\mathbf{v}_{\mathrm{b}} \\
\mathbf{w}_{\mathrm{b}}
\end{array}\right]+\left[\begin{array}{c}
\mathbf{J}_{\mathrm{T} w} \\
\mathbf{H}_{\mathrm{w} \phi}
\end{array}\right] \dot{\phi}_{\mathrm{m}}
$$

where

$$
\begin{aligned}
& \mathbf{H}_{\mathrm{w}}=\left[\sum_{i=1}^{m}\left(\mathbf{I}_{i}+m_{i} \mathbf{r}_{0 i}^{\times \mathrm{T}} \mathbf{r}_{0 i}^{\times}\right)+\mathbf{I}_{0}\right] \in \mathbb{R}^{3 \times 3} \\
& \mathbf{H}_{\mathrm{w} \phi}=\left[\sum_{i=1}^{m}\left(\mathbf{I}_{i} \mathbf{J}_{\mathrm{R} i}+m_{i} \mathbf{r}_{0 i}^{\times} \mathbf{J}_{\mathrm{T} i}\right)\right] \in \mathbb{R}^{3 \times m}
\end{aligned}
$$

The compact form of the servicer's linear and angular momentum in $(2.18)$ will be utilized 
in the formulation of the servicer's equation of motion. In this compact form, the coefficient matrix of the servicer's base linear and angular velocity is the servicer's base inertia matrix, $\mathbf{H}_{\mathrm{b}}$. The coefficient matrix of the servicer's manipulator joint rates is the inertia coupling matrix, $\mathbf{H}_{\mathbf{c}}$. These matrices are defined as follows:

$$
\begin{gathered}
\mathbf{H}_{\mathrm{b}}=\left[\begin{array}{cc}
m_{\text {total }} \mathbf{E}_{3} & m_{\text {total }} \mathbf{r}_{0 \mathrm{~g}}^{\times \mathrm{T}} \\
m_{\text {total }} \mathbf{r}_{0 \mathrm{~g}}^{\times} & \mathbf{H}_{\mathrm{w}}^{\times}
\end{array}\right] \in \mathbb{R}^{6 \times 6} \\
\mathbf{H}_{\mathrm{c}}=\left[\begin{array}{c}
\mathbf{J}_{\mathrm{T} w} \\
\mathbf{H}_{\mathrm{w} \phi}
\end{array}\right] \in \mathbb{R}^{6 \times m} .
\end{gathered}
$$

\subsection{Servicer Dynamics}

In this Section, the dynamics of a space robot is reviewed in order to obtain the equations of motion in the operation space of the space robot similar to the derivation by [47]. The space robot consists of a base body and $m$-link serial manipulator arm (Fig. 2.1).

Let $\boldsymbol{v}_{\mathrm{b}}=\left[\mathbf{v}_{\mathrm{b}}^{\mathrm{T}}, \mathbf{w}_{\mathrm{b}}^{\mathrm{T}}\right]^{\mathrm{T}} \in \mathbb{R}^{6}$ and $\dot{\phi}_{\mathrm{m}} \in \mathbb{R}^{m}$ represent the linear and angular velocity of the servicer's base center of mass and joint rates of the manipulator, respectively. The equation of motion of the servicer system is given by:

$$
\mathbf{H}_{\gamma}\left[\begin{array}{c}
\dot{\boldsymbol{v}}_{\mathrm{b}} \\
\ddot{\phi}_{\mathrm{m}}
\end{array}\right]+\mathbf{c}_{\gamma}=\left[\begin{array}{c}
\mathbf{f}_{\mathrm{b}} \\
\tau_{\mathrm{m}}
\end{array}\right]+\left[\begin{array}{c}
\mathbf{J}_{\mathrm{b}}^{\mathrm{T}} \\
\mathbf{J}_{\mathrm{m}}^{\mathrm{T}}
\end{array}\right]\left(-\mathbf{f}_{\mathrm{e}}\right)
$$

where $\mathbf{H}_{\gamma} \in \mathbb{R}^{(6+m) \times(6+m)}$ is the generalized mass matrix of the servicer system ( [68]) and $\mathbf{c}_{\gamma} \in$ $\mathbb{R}^{(6+m)}$ represents the non-linear velocity dependent terms. $\mathbf{f}_{\mathrm{e}}=\left[\mathbf{F}_{\mathrm{e}}^{\mathrm{T}}, \tau_{\mathrm{e}}^{\mathrm{T}}\right]^{\mathrm{T}} \in \mathbb{R}^{6}$ represents the force/torque at the end-effector where $\mathbf{F}_{\mathrm{e}} \in \mathbb{R}^{3}$ denotes the force and $\tau_{\mathrm{e}} \in \mathbb{R}^{3}$ denotes the torque. $\tau_{\mathrm{m}} \in \mathbb{R}^{m}$ denotes the manipulator's joint torques. $\mathbf{f}_{\mathrm{b}}=\left[\mathbf{F}_{\mathrm{b}}^{\mathrm{T}}, \tau_{\mathrm{b}}^{\mathrm{T}}\right]^{\mathrm{T}} \in \mathbb{R}^{6}$ are the force/torque applied to the base center of mass, where $\mathbf{F}_{\mathrm{b}}=0$ (Assumption 3, no linear momentum) and $\tau_{\mathrm{b}}$ is the control torque to be applied by the servicer's base attitude control system. $\mathbf{J}_{\mathrm{b}} \in \mathbb{R}^{6 \times 6}$ and $\mathbf{J}_{\mathrm{m}} \in \mathbb{R}^{6 \times m}$ are the servicer's base and manipulator Jacobian matrices and are defined in $2.8 \mathrm{~b}$ and (2.8c), respectively. The matrices $\mathbf{H}_{\gamma}$ is defined as follows:

$$
\mathbf{H}_{\gamma}=\left[\begin{array}{ll}
\mathbf{H}_{\mathrm{b}} & \mathbf{H}_{\mathrm{c}} \\
\mathbf{H}_{\mathrm{c}}^{\mathrm{T}} & \mathbf{H}_{\phi}
\end{array}\right]
$$


where $\mathbf{H}_{\mathrm{b}}$ is defined in $(2.21), \mathbf{H}_{\mathrm{c}}$ is defined in $(2.22)$, and $\mathbf{H}_{\phi}$ is the mass matrix of the manipulator and is defined as follows [68]:

$$
\mathbf{H}_{\phi}=\sum_{i=1}^{m}\left(\mathbf{J}_{\mathrm{R} i}^{\mathrm{T}} \mathbf{I}_{i} \mathbf{J}_{\mathrm{R} i}+m_{i} \mathbf{J}_{\mathrm{T} i}^{\mathrm{T}} \mathbf{J}_{\mathrm{T} i}\right)
$$

The servicer's base linear acceleration, $\dot{\mathbf{v}}_{\mathrm{b}}$, can be eliminated from 2.23 , resulting in:

$$
\mathbf{M}\left[\begin{array}{l}
\ddot{\phi}_{\mathrm{m}} \\
\dot{\mathbf{w}}_{\mathrm{b}}
\end{array}\right]+\mathbf{c}=\left[\begin{array}{c}
\tau_{\mathrm{m}} \\
\tau_{\mathrm{b}}
\end{array}\right]+\mathbf{J}\left(-\mathbf{f}_{\mathrm{e}}\right)
$$

where the submatrices $\mathbf{M} \in \mathbb{R}^{(m+3) \times(m+3)}, \mathbf{c} \in \mathbb{R}^{(m+3)}$ and $\mathbf{J} \in \mathbb{R}^{(m+3) \times 6}$ result from the above stated elimination [42]. Additional modifications can be made to 2.26 in order to express the servicer dynamics in task space. This can be achieved by utilizing the momentum of the servicer system [68]:

$$
\left[\begin{array}{c}
\mathbf{P}_{\mathrm{b}} \\
\mathbf{L}_{\mathrm{b}}
\end{array}\right]=\mathbf{H}_{\mathrm{b}} \boldsymbol{v}_{\mathrm{b}}+\mathbf{H}_{\mathrm{c}} \dot{\phi}
$$

where $\mathbf{P}_{\mathrm{b}} \in \mathbb{R}^{3}$ and $\mathbf{L}_{\mathrm{b}} \in \mathbb{R}^{3}$ are the inertial linear and angular momentum of the servicer about the base's center of mass and are defined in 2.18). $\mathbf{H}_{\mathrm{b}} \in \mathbb{R}^{6 \times 6}$ is the base inertia matrix and $\mathbf{H}_{\mathrm{c}} \in \mathbb{R}^{6 \times m}$ is the inertial coupling matrix and are defined in 2.21) and 2.22, respectively. Furthermore, recall from (2.7) the kinematic relationship between the servicer's end-effector, base and manipulator joint velocities:

$$
v_{\mathrm{e}}=\mathbf{J}_{\mathrm{b}} v_{\mathrm{b}}+\mathbf{J}_{\mathrm{m}} \dot{\phi}_{\mathrm{m}}
$$

where $\boldsymbol{v}_{\mathrm{e}}=\left[\mathbf{v}_{\mathrm{e}}^{\mathrm{T}}, \mathbf{w}_{\mathrm{e}}^{\mathrm{T}}\right]^{\mathrm{T}} \in \mathbb{R}^{6}$ represents the end-effector's linear and angular velocity. The base's linear velocity can be eliminated from (2.28) with the use of servicer's linear momentum in (2.27) and results in the following [47]:

$$
v_{\mathrm{e}}=\mathbf{A}_{\mathrm{b}} \mathbf{w}_{\mathrm{b}}+\mathbf{A}_{\mathrm{m}} \dot{\phi}_{\mathrm{m}}+\frac{1}{m_{\text {total }}} \mathbf{P}_{\mathrm{b}}
$$

where $m_{\text {total }}$ is the total mass of the servicer system, $\mathbf{A}_{\mathbf{b}} \in \mathbb{R}^{6 \times 3}$ and $\mathbf{A}_{\mathrm{m}} \in \mathbb{R}^{6 \times m}$ are defined 
in [47] and presented below for convenience. The matrix $\mathbf{A}_{\mathrm{b}}$ is defined as follows:

$$
\mathbf{A}_{\mathrm{b}}=\left[\begin{array}{c}
\mathbf{r}_{\mathrm{ge}}^{\times} \\
\mathbf{E}_{3}
\end{array}\right] \in \mathbb{R}^{6 \times 3}
$$

where $\mathbf{r}_{\text {ge }} \in \mathbb{R}^{3}$ is a position vector from the center of mass of the servicer system to the endeffector. The matrix $\mathbf{A}_{\mathrm{m}}$ is defined as follows:

$$
\mathbf{A}_{\mathrm{m}}=\left[\begin{array}{c}
\mathbf{J}_{\mathrm{m}}^{\mathrm{t}}-\frac{1}{m_{\text {total }}} \mathbf{J}_{\mathrm{Tw}} \\
\mathbf{J}_{\mathrm{m}}^{\mathrm{r}}
\end{array}\right] \in \mathbb{R}^{6 \times m}
$$

where $\mathbf{J}_{\mathrm{m}}^{\mathrm{t}} \in \mathbb{R}^{3 \times m}$ and $\mathbf{J}_{\mathrm{m}}^{\mathrm{r}} \in \mathbb{R}^{3 \times m}$ are the linear and rotational component of the manipulator Jacobian, $\mathbf{J}_{\mathrm{m}}$, defined in 2.8c): $\mathbf{J}_{\mathrm{m}}=\left[\mathbf{J}_{\mathrm{m}}^{\mathrm{t}}, \mathbf{J}_{\mathrm{m}}^{\mathrm{r}}\right]^{\mathrm{T}}$. $\mathbf{J}_{\mathrm{Tw}}$ is defined in 2.12d .

Taking the time derivative of 2.29) and solving for the manipulator joint acceleration, $\ddot{\phi}_{\mathrm{m}}$, results in the following:

$$
\ddot{\phi}_{\mathrm{m}}=\mathbf{A}_{\mathrm{m}}^{+}\left(\dot{\boldsymbol{v}}_{\mathrm{e}}-\mathbf{A}_{\mathrm{b}} \dot{\mathbf{w}}_{\mathrm{b}}-\mathbf{c}_{\mathrm{A}}-\frac{1}{m_{\text {total }}}\left[\begin{array}{cc}
\mathbf{E}_{3} & \mathbf{0} \\
\mathbf{0} & \mathbf{0}
\end{array}\right] \mathbf{f}_{\mathrm{e}}\right)+\mathbf{P}_{\mathrm{ND}} \eta
$$

where $\mathbf{c}_{\mathrm{A}}=\left(\dot{\mathbf{A}}_{\mathrm{b}} \mathbf{w}_{\mathrm{b}}+\dot{\mathbf{A}}_{\mathrm{m}} \dot{\phi}_{\mathrm{m}}\right) \in \mathbb{R}^{6}$ and the product $\mathbf{P}_{\mathrm{ND}} \eta$ represent manipulator joint acceleration in the null space of $\mathbf{A}_{\mathrm{m}}$, denoted as $N\left(\mathbf{A}_{\mathrm{m}}\right) . \mathbf{P}_{\mathrm{ND}}=\left(\mathbf{E}_{m}-\mathbf{A}_{\mathrm{m}}^{+} \mathbf{A}_{\mathrm{m}}\right) \in \mathbb{R}^{m \times m}$ is the projection operator onto $N\left(\mathbf{A}_{\mathrm{m}}\right)$ and $\eta \in \mathbb{R}^{m}$ can be designed to accomplish a task in $N\left(\mathbf{A}_{\mathrm{m}}\right)$. Any task carried out in $N\left(\mathbf{A}_{\mathrm{m}}\right)$ will not affect the end-effector's acceleration and hence will not influence the end-effector's position/orientation [4,14]. $N\left(\mathbf{A}_{\mathrm{m}}\right)$ is non-trivial as long as the manipulator arm is redundant with respect to the end-effector's task space representation, that is $m>6$, and $\operatorname{rank}\left(\mathbf{A}_{\mathrm{m}}\right)=6$.

To arrive at the final equation of motion of the servicer, (2.32) is utilized to express the manipulator joint acceleration and base angular acceleration $\left(\left[\ddot{\phi}_{\mathrm{m}}^{\mathrm{T}}, \dot{\mathbf{w}}_{\mathrm{b}}^{\mathrm{T}}\right]^{\mathrm{T}}\right)$ as follows:

$$
\left[\begin{array}{c}
\ddot{\phi}_{\mathrm{m}} \\
\dot{\mathbf{w}}_{\mathrm{b}}
\end{array}\right]=\mathbf{A}\left[\begin{array}{c}
\dot{\boldsymbol{v}}_{\mathrm{e}} \\
\dot{\mathbf{w}}_{\mathrm{b}}
\end{array}\right]+\overline{\mathbf{c}}_{\mathrm{A}}+\mathbf{J}_{\mathrm{A}}\left(-\mathbf{f}_{\mathrm{e}}\right)+\left[\begin{array}{c}
\mathbf{P}_{\mathrm{ND}} \eta \\
\mathbf{0}
\end{array}\right]
$$

where the matrices $\mathbf{A} \in \mathbb{R}^{(m+3) \times(6+3)}, \overline{\mathbf{c}}_{\mathrm{A}} \in \mathbb{R}^{(m+3)}$ and $\mathbf{J}_{\mathrm{A}} \in \mathbb{R}^{(m+3) \times(6)}$ are defined in [47] and 
are presented here for completeness. The terms $\mathbf{A}, \mathbf{c}_{\mathrm{A}}$ and $\mathbf{J}_{\mathrm{A}}$ in 2.33 are defined as follows:

$$
\begin{gathered}
\mathbf{A}=\left[\begin{array}{cc}
\mathbf{A}_{\mathrm{m}}^{+} & -\mathbf{A}_{\mathrm{m}}^{+} \mathbf{A}_{\mathrm{b}} \\
\mathbf{0} & \mathbf{E}_{3}
\end{array}\right] \quad \overline{\mathbf{c}}_{\mathrm{A}}=\left[\begin{array}{c}
\mathbf{A}_{\mathrm{m}}^{+} \mathbf{c}_{\mathrm{A}} \\
\mathbf{0}
\end{array}\right] \\
\mathbf{J}_{\mathrm{A}}=\left[\begin{array}{c}
\mathbf{A}_{\mathrm{m}}^{+}\left[\begin{array}{cc}
\mathbf{E}_{3} \frac{-1}{m_{\text {total }}} & \mathbf{0} \\
\mathbf{0} & \mathbf{0}
\end{array}\right] \\
\mathbf{0}
\end{array}\right]
\end{gathered}
$$

Substituting 2.33 into 2.26 results in the following:

$$
\overline{\mathbf{M}}\left[\begin{array}{c}
\dot{\boldsymbol{v}}_{\mathrm{e}} \\
\dot{\mathbf{w}}_{\mathrm{b}}
\end{array}\right]+\overline{\mathbf{c}}=\left[\begin{array}{c}
\tau_{\mathrm{m}} \\
\tau_{\mathrm{b}}
\end{array}\right]+\overline{\mathbf{J}}\left(-\mathbf{f}_{\mathrm{e}}\right)-\mathbf{M}\left[\begin{array}{c}
\mathbf{P}_{\mathrm{ND}} \eta \\
\mathbf{0}
\end{array}\right]
$$

where $\overline{\mathbf{M}}=\mathbf{M A} \in \mathbb{R}^{(m+3) \times(6+3)}, \overline{\mathbf{c}}=\left(\mathbf{M} \overline{\mathbf{c}}_{\mathrm{A}}+\mathbf{c}\right) \in \mathbb{R}^{(m+3)}$ and $\overline{\mathbf{J}}=\left(\mathbf{J}-\mathbf{M} \mathbf{J}_{\mathrm{A}}\right) \in \mathbb{R}^{(m+3) \times 6}$.

Furthermore, pre-multiply 2.36 by $\mathbf{A}^{\mathrm{T}}$ results in the following:

$$
\mathbf{M}_{\mathrm{s}} \dot{\xi}+\mathbf{c}_{\mathrm{s}}=\mathbf{A}^{\mathrm{T}} \tau+\mathbf{J}_{\mathrm{s}}\left(-\mathbf{f}_{\mathrm{e}}\right)-\mathbf{A}^{\mathrm{T}} \mathbf{M}\left[\begin{array}{c}
\mathbf{P}_{\mathrm{ND}} \eta \\
\mathbf{0}
\end{array}\right]
$$

where $\xi=\left[\boldsymbol{v}_{\mathrm{e}}^{\mathrm{T}}, \mathbf{w}_{\mathrm{b}}^{\mathrm{T}}\right]^{\mathrm{T}}, \mathbf{M}_{\mathrm{s}}=\mathbf{A}^{\mathrm{T}} \mathbf{M A} \in \mathbb{R}^{(6+3) \times(6+3)}, \mathbf{c}_{\mathrm{s}}=\mathbf{A}^{\mathrm{T}}\left(\mathbf{M} \overline{\mathbf{c}}_{\mathrm{A}}+\mathbf{c}\right) \in \mathbb{R}^{(6+3)}, \mathbf{J}_{\mathrm{s}}=\mathbf{A}^{\mathrm{T}}(\mathbf{J}-$ $\left.\mathbf{M} \mathbf{J}_{\mathrm{A}}\right) \in \mathbb{R}^{(6+3) \times 6}$ and $\tau=\left[\tau_{\mathrm{m}}^{\mathrm{T}}, \tau_{\mathrm{b}}^{\mathrm{T}}\right]^{\mathrm{T}}$.

\subsection{Target Dynamics}

The target is modeled as a single rigid body where $\boldsymbol{v}_{\mathrm{t}}=\left[\mathbf{v}_{\mathrm{t}}^{\mathrm{T}}, \mathbf{w}_{\mathrm{t}}^{\mathrm{T}}\right]^{\mathrm{T}} \in \mathbb{R}^{6}$ represents the target's center of mass linear and angular velocity. The equation of motion of the target is given by:

$$
\mathbf{M}_{\mathrm{t}} \dot{\boldsymbol{v}}_{\mathrm{t}}+\mathbf{c}_{\mathrm{t}}=\mathbf{J}_{\mathrm{t}}^{\mathrm{T}}\left(\mathbf{f}_{\mathrm{e}}\right)
$$

where $\mathbf{M}_{\mathrm{t}} \in \mathbb{R}^{6 \times 6}, \mathbf{c}_{\mathrm{t}} \in \mathbb{R}^{6}$ and $\mathbf{J}_{\mathrm{t}} \in \mathbb{R}^{6 \times 6}$ are defined as:

$$
\mathbf{M}_{\mathrm{t}}=\left[\begin{array}{cc}
m_{\mathrm{t}} \mathbf{E}_{3} & \mathbf{0} \\
\mathbf{0} & \mathbf{I}_{\mathrm{t}}
\end{array}\right] \quad \mathbf{c}_{\mathrm{t}}=\left[\begin{array}{c}
\mathbf{0} \\
\mathbf{w}_{\mathrm{t}} \times \mathbf{I}_{\mathrm{t}} \mathbf{w}_{\mathrm{t}}
\end{array}\right] \quad \mathbf{J}_{\mathrm{t}}=\left[\begin{array}{cc}
\mathbf{E}_{3} & -\mathbf{r}_{\mathrm{te}}^{\times} \\
\mathbf{0} & \mathbf{E}_{3}
\end{array}\right]
$$


where $m_{\mathrm{t}}$ and $\mathbf{I}_{\mathbf{t}} \in \mathbb{R}^{3 \times 3}$ are the mass and inertia tensor of the target, respectively. $\mathbf{r}_{\mathrm{te}} \in \mathbb{R}^{3}$ (Fig. 1 ) is the radial vector from the target's center of mass to the grasping point.

The velocity of the end-effector and target are subjected to the following kinematic constraints as a result of grasping (assumption 2):

$$
\begin{gathered}
v_{\mathrm{e}}=\mathbf{J}_{\mathrm{t}} \boldsymbol{v}_{\mathrm{t}} \\
\dot{\boldsymbol{v}}_{\mathrm{e}}=\mathbf{J}_{\mathrm{t}} \dot{\boldsymbol{v}}_{\mathrm{t}}+\dot{\mathbf{J}}_{\mathrm{t}} \boldsymbol{v}_{\mathrm{t}}
\end{gathered}
$$

Utilizing (2.40) and (2.41) in (2.38), the target's dynamics can be expressed as:

$$
\Lambda_{\mathrm{t}} \dot{v}_{\mathrm{e}}-\Lambda_{\mathrm{t}} \dot{\mathbf{J}}_{\mathrm{t}} \mathbf{J}_{\mathrm{t}}^{-1} v_{\mathrm{e}}+\mathbf{J}_{\mathrm{t}}^{-\mathrm{T}} \mathbf{c}_{\mathrm{t}}=\mathbf{f}_{\mathrm{e}}
$$

where $\Lambda_{t}=\mathbf{J}_{\mathrm{t}}^{-\mathrm{T}} \mathbf{M}_{\mathrm{t}} \mathbf{J}_{\mathrm{t}}^{-1}$ represents the target's inertia projected onto the end-effector.

\subsection{Combined Servicer-Target Dynamics}

The equation of motion of the combined servicer and target system is obtained by substituting (2.42) into 2.37), resulting in the following:

$$
\overline{\mathbf{M}} \dot{\xi}+\overline{\mathbf{c}}=\mathbf{A}^{\mathrm{T}} \tau
$$

where $\overline{\mathbf{M}}=\mathbf{M}_{\mathrm{s}}+\left[\begin{array}{ll}\mathbf{J}_{\mathrm{s}} \Lambda_{\mathrm{t}} & \mathbf{0}\end{array}\right]$ and $\overline{\mathbf{c}}=\mathbf{c}_{\mathrm{s}}+\mathbf{J}_{\mathrm{s}}\left(\mathbf{J}_{\mathrm{t}}^{-\mathrm{T}} \mathbf{c}_{\mathrm{t}}-\Lambda_{\mathrm{t}} \dot{\mathbf{J}}_{\mathrm{t}} \mathbf{J}_{\mathrm{t}}^{-1} \boldsymbol{v}_{\mathrm{e}}\right)$. The equation of motion of the combined servicer and target system, 2.43), is utilized in the formulation of the tracking control based detumbling strategy and assumes that the servicer does not contain any redundancy with respect to the detumbling task.

\subsection{Control Objective}

The goal of this study is to develop a detumbling control strategy for the servicer to detumble a non-cooperative tumbling target in the post-grasping phase. Detumbling of the target requires that the target's tumbling motion be brought to rest while rejecting the target's gained 
momentum. Furthermore, this must be accomplished without exceeding force/torque limits at the servicer's end-effector and without accurate knowledge of the target's inertial parameters.

The target's inertial parameters consists of its mass $\left(m_{\mathfrak{t}}\right)$, inertia tensor $\left(\mathbf{I}_{\mathbf{t}}\right)$ and location of center of mass from the grasping location $\left(\mathbf{r}_{\mathrm{te}}\right)$. These parameters cannot be assumed known prior to the post-grasping phase as there is no practical way to measure remaining propellant fuel in zero gravity. The target (i.e., malfunctioning satellite) is not actuated by any onboard actuators and hence has no means of expending fuel in the post-grasping phase. This implies that its mass, bounds on its inertia tensor $\left(\lambda_{\min }\left(\mathbf{I}_{t}\right) \leq\left\|\mathbf{I}_{t}\right\| \leq \lambda_{\max }\left(\mathbf{I}_{t}\right)\right)$, and magnitude of its location of center of mass from the grasping point $\left(\left\|\mathbf{r}_{\mathrm{te}}\right\|\right)$ are fixed and do not change during the post-grasping phase. However, these bounds are not known and must be estimated prior to the post-grasping phase. To determine these estimates, consider the following inequalities:

$$
\begin{gathered}
m_{\mathrm{tL}} \leq m_{\mathrm{t}} \leq m_{\mathrm{tU}} \\
\lambda_{\operatorname{maxL}} \leq \lambda_{\max }\left(\mathbf{I}_{\mathrm{t}}\right) \leq \lambda_{\operatorname{maxU}} \\
\lambda_{\operatorname{minL}} \leq \lambda_{\min }\left(\mathbf{I}_{\mathrm{t}}\right) \leq \lambda_{\operatorname{minU}} \\
r_{\mathrm{teL}} \leq\left\|\mathbf{r}_{\mathrm{te}}\right\| \leq r_{\mathrm{teU}}
\end{gathered}
$$

The upper and lower estimates of bounds on the target's inertial parameters, denoted as $(\cdot)_{(\cdot) \mathrm{L}}$ and $(\cdot)_{(\cdot) \mathrm{U}}$ in 2.44), can be estimated based on pre-launch data of the target satellite. Prelaunch data of the satellite can be utilized to determine the satellites mass, inertia tensor and location of center of mass with and without fuel. As a result of this, bounds on the target's inertial parameters and their estimates will depend on the target in question due differences in the target's inertial parameters with and without fuel. For instance, the percentage of a satellites fuel mass to its total mass (satellites mass with fuel) can vary from $0 \%$ to $50 \%$ and ultimately depends on the satellite's mission [36].

Force/torque limits at the servicer's end-effector play an important role in the post-grasping phase. Force/torque limits can be specified based on design limits of the servicer's end-effector grasping mechanism [20,26], structural limits of the grasping point on the target [7,9,47,65,66] and magnitude limits on the servicer's control inputs. End-effector's force/torque limits are 
related to the magnitude limits on the servicer's control inputs so as to ensure that the target's momentum can be rejected by the servicer's control inputs as it is transferred from the target to the servcier. An increase in the end-effector's force/torque limits will result in larger transfer of momentum from the target to the servicer and will require larger servicer's control inputs to rejected the gained momentum so as to avoid tumbling of the combined servicer-target system.

Bringing the tumbling target's motion to rest is reflected in its center of mass linear and angular velocity $\left(v_{\mathrm{t}}\right)$ converging to zero. However, as per the kinematic constraints presented in (2.40) and 2.41) relating $\boldsymbol{v}_{\mathrm{t}}$ to $\boldsymbol{v}_{\mathrm{e}}$, detumbling of the target can be realized once the servicer's end-effector linear and angular velocity $\left(\boldsymbol{v}_{\mathrm{e}}\right)$ converge to zero. As stated above, it is required that the target's momentum be rejected in order to avoid tumbling of the combined servicer-target system as the end-effector's linear and angular velocity converge to zero. Under assumption 3, the servicer-target system will only contain angular momentum. Rejection of the target's angular momentum in the post-grasping phase can be realized by requiring that the servicer's end-effector linear and angular velocity and the servicer's base angular velocity, $\xi=\left[v_{\mathrm{e}}^{\mathrm{T}}, \mathbf{w}_{\mathrm{b}}^{\mathrm{T}}\right]^{\mathrm{T}}$, converge to zero. This will mark the completion of the post-grasping phase.

End-effector force/torque limits can be expressed as follows: $\left\|\mathbf{F}_{\mathrm{e}}\right\| \leq \mathrm{F}_{\max }$, and $\left\|\tau_{\mathrm{e}}\right\| \leq$ $\tau_{\max }$, where $\mathrm{F}_{\max }$ and $\tau_{\max }$ are the specified force and torque limits, respectively. The interaction force/torque experienced at the end-effector is dependent on the servicer's and target's inertial parameters. While the servicer's inertial parameters are assumed known, the target's inertial parameters are not known prior to the post-grasping phase but are bounded.

The control objective of this study is to design the servicer's control input for its manipulator and base attitude control system, $\tau=\left[\tau_{\mathrm{m}}^{\mathrm{T}}, \tau_{\mathrm{b}}^{\mathrm{T}}\right]^{\mathrm{T}}$, that will bring the servicer's end-effector linear and angular velocity and servicer's base angular velocity, $\xi$, to zero subjected to specified end-effector force and torque limits, $\mathrm{F}_{\max }$ and $\tau_{\max }$. 


\section{Chapter 3}

\section{Detumbling Strategy using Force/Torque Measurements}

\subsection{Detumbling Strategy Overview}

In this Chapter, a detumbling control strategy is presented for the servicer to detumble a noncooperative target with unknown inertial parameter, while being subjected to force/torque limits at the servicer's end-effector. The presented detumbling control strategy is formulated under the assumption that end-effector force/torque measurements are available. Detumbling is achieved by following a newly defined reference detumbling force/torque that is designed to bring the target's linear and angular velocities to zero, while respecting force/torque limits at the servicer's end-effector. The reference detumbling force/torque is computed as a function of known servicer parameters (end-effector force/torque limit and end-effector's linear and angular velocities) and do not require the use of the target's inertial parameters in its formulation as in [6, 7, 9, 18, 65, 72]. The proposed detumbling control strategy enables the servicer to apply a force/torque to the target's grasping point that will bring its tumbling motion to rest while allowing the servicer to comply with the targets' unknown residual tumbling motion.

The controller to detumble the target is presented as follows:

$$
\left[\begin{array}{c}
\tau_{\mathrm{m}} \\
\tau_{\mathrm{b}}
\end{array}\right]=\overline{\mathbf{c}}+\overline{\mathbf{J}} \mathbf{f}_{\mathrm{e}}+\overline{\mathbf{M}}\left[\begin{array}{c}
\mathbf{u}_{\mathrm{f}} \\
\mathbf{u}_{\mathrm{b}}
\end{array}\right]
$$


where $\mathbf{u}_{\mathrm{f}} \in \mathbb{R}^{6}$ and $\mathbf{u}_{\mathrm{b}} \in \mathbb{R}^{3}$ are auxiliary inputs that will be designed to detumble the target and to stabilize the servicer's base, respectively. Note, it was assumed the servicer does not possess any redundancy with respect to the detumbling task.

Substituting (3.1) into 2.36) will result in the following:

$$
\left[\begin{array}{c}
\dot{\boldsymbol{v}}_{\mathrm{e}} \\
\dot{\mathbf{w}}_{\mathrm{b}}
\end{array}\right]=\left[\begin{array}{c}
\mathbf{u}_{\mathrm{f}} \\
\mathbf{u}_{\mathrm{b}}
\end{array}\right] .
$$

The auxiliary inputs in (3.2) will be designed to bring the target's tumbling motion to rest subjected to end-effector force/torque limits while rejecting the target's gained momentum. First, the design of the auxiliary input $\mathbf{u}_{\mathrm{b}}$ will be presented to control the servicer's base attitude in order to reject the target's gained momentum as its tumbling motion is brought to rest. Following this, the design of $\mathbf{u}_{\mathrm{f}}$ will be presented to dampen the target's motion while allowing compliance with its residual unknown tumbling motion. The development of $\mathbf{u}_{\mathrm{f}}$ will be presented in two stages. In the first stage, the reference force/toque will be presented. In this stage $\mathbf{u}_{\mathrm{f}}$ will be designed to follow the reference force/torque without accounting for changes in the servicer's inertial parameters as a result of the captured target. Instead, bounds on the target's inertial parameters and tumbling rate are derived for which the approach can detumble (domain of attraction). In the second stage, an adaptive robust compensator will be introduced into the design of $\mathbf{u}_{\mathrm{f}}$ that will account for changes in the servicer's inertial parameters due to the captured unknown target, and its benefits will be highlighted.

\subsection{Control of Servicer's Base Attitude}

In the post-grasping phase, the target's momentum is transferred to the servicer as its tumbling motion is brought to rest. The gained momentum must be rejected by the servicer in order to avoid tumbling of the combined servicer/target system. This can be achieved simply by utilizing the following controller:

$$
\mathbf{u}_{\mathrm{b}}=-\mathbf{K}_{\mathrm{AD}} \mathbf{w}_{\mathrm{b}}
$$

where $\mathbf{K}_{\mathrm{AD}} \in \mathbb{R}^{3 \times 3}$ is positive definite. As per 3.1, control of the base and servicer's endeffector are decoupled. This allows the use of any controller to control the servicer's base. For 
instance, if it is desirable to maintain the attitude of the servicer's base to a specific attitude or to re-orientate the attitude during the detumbling procedure, a simple PD quaternion feedback can be utilized as follows:

$$
\mathbf{u}_{\mathrm{b}}=-\mathbf{K}_{\mathrm{AD}} \mathbf{w}_{\mathrm{b}}-\mathrm{K}_{\mathrm{AP}} \delta \mathbf{q}_{\mathrm{v}}
$$

where $\mathrm{K}_{\mathrm{AP}}>0 \in \mathbb{R} . \delta \mathbf{q}_{\mathrm{v}}=\mathrm{q}_{\mathrm{s}} \mathbf{q}_{\mathrm{v}}^{*}+\mathbf{q}_{\mathrm{v}} \times \mathbf{q}_{\mathrm{v}}^{*}-\mathbf{q}_{\mathrm{v}} \mathrm{q}_{\mathrm{s}}^{*} \in \mathbb{R}^{3}$ represents the vector component of the quaternion error where the scalar and vector components of the quaternion are denoted by $(\cdot)_{\mathrm{s}}$ and $(\cdot)_{\mathrm{v}}$, respectively, and $(\cdot)^{*}$ represents the desired quaternion value. The utilization of either controllers for the servicer's base, 3.3a or 3.3b), will result in the rejection of the gained target's momentum as it is brought to rest.

\subsection{Control of Servicer's End-effector}

A force/torque must be applied to the target to counteract its momentum and bring it to rest. In [7] and [9], knowledge of the target's inertial parameters allowed the formulation of a detumbling trajectory that adhere to force/torque limits at the grasping location. The force/torque controller presented in this section allows compliance with the target while damping its motion subjected to force/torque limits. This is achieved by applying a force/torque at the grasping point that is opposite in direction to the end-effector's linear and angular velocities and scaled by the force/torque limits, respectively. The end-effector's desired force/torque, denoted as $\mathbf{f}_{\mathrm{eD}}=\left[\mathbf{F}_{\mathrm{eD}}^{\mathrm{T}}, \tau_{\mathrm{eD}}^{\mathrm{T}}\right]^{\mathrm{T}} \in \mathbb{R}^{6 \times 1}$, are defined as:

$$
\begin{gathered}
\mathbf{F}_{\mathrm{eD}}=\left\{\begin{array}{cc}
\frac{\mathbf{v}_{\mathrm{e}}}{\left\|\mathbf{v}_{\mathrm{e}}\right\|}\left(-\mathrm{F}_{\max }\right) & \left\|\mathbf{v}_{\mathrm{e}}\right\|>0 \\
0 & \mathbf{v}_{\mathrm{e}}=0
\end{array}\right. \\
\tau_{\mathrm{eD}}=\left\{\begin{array}{cc}
\mathbf{w}_{\mathrm{e}}\left(-\tau_{\mathrm{max}}\right) & \left\|\mathbf{w}_{\mathrm{e}}\right\|>0 \\
0 & \mathbf{w}_{\mathrm{e}}=0
\end{array}\right.
\end{gathered}
$$

where $\mathrm{F}_{\max }$ and $\tau_{\max }$ are the limits of the end-effector's force and torque magnitude and are based on the structural design of the manipulator.

The auxiliary input, $\mathbf{u}_{\mathrm{f}}$ is defined as follows to track the desired force/torque [39]:

$$
\mathbf{u}_{\mathrm{f}}=\mathbf{K}_{\mathrm{fP}}\left(\mathbf{f}_{\mathrm{eD}}-\mathbf{f}_{\mathrm{e}}\right)
$$


where $\mathbf{K}_{\mathrm{fP}} \in \mathbb{R}^{6 \times 6}>0$ is block diagonal proportional error gains, respectively.

\subsubsection{Controller Evaluation}

Substituting (3.3b) and (3.6) into (3.2) results in the following closed-loop equations:

$$
\begin{gathered}
\dot{\boldsymbol{v}}_{\mathrm{e}}=\mathbf{K}_{\mathrm{fP}}\left(\mathbf{f}_{\mathrm{eD}}-\mathbf{f}_{\mathrm{e}}\right) \\
\dot{\mathbf{w}}_{\mathrm{b}}=-\mathbf{K}_{\mathrm{AD}} \mathbf{w}_{\mathrm{b}}-\mathrm{K}_{\mathrm{AP}} \delta \mathbf{q}_{\mathrm{v}}
\end{gathered}
$$

Stability of the base attitude controller, (3.8), is proven based on the Lyapunov argument in [73]. The closed loop dynamics of (3.7) describes compliance of the end-effector subjected to the force error on the right side of (3.7). The dynamics of the environment must be utilized to draw conclusions about the stability of (3.7). Under the current application of detumbling a non-cooperative target, the dynamics of the environment is established by the tumbling target and stability of the force controller in (3.7) can be ascertained by considering the environmental force, $\mathbf{f}_{\mathrm{e}}$ (target's dynamics).

Utilizing (3.4) and (3.5), 3.7) can be expressed as:

$$
\dot{\boldsymbol{v}}_{\mathrm{e}}+\mathbf{K}_{\mathrm{fP}}\left[\begin{array}{cc}
\frac{\mathrm{F}_{\max }}{\left\|\mathbf{v}_{\mathrm{e}}\right\|} \mathbf{E}_{3} & \mathbf{0} \\
\mathbf{0} & \frac{\tau_{\max }}{\left\|\mathbf{v}_{\mathrm{e}}\right\|} \mathbf{E}_{3}
\end{array}\right] \boldsymbol{v}_{\mathrm{e}}=-\mathbf{K}_{\mathrm{fP}} \mathbf{f}_{\mathrm{e}}
$$

for $\left\|\mathbf{v}_{\mathrm{e}}\right\|>0$ and $\left\|\mathbf{w}_{\mathrm{e}}\right\|>0$. The above equation demonstrates that the interacting system is stable since the excitation to the system, $-\mathbf{K}_{\mathrm{fP}} \mathbf{f}_{\mathrm{e}}$, is generated entirely by passive elements [11]: the target has no means of creating energy.

During the detumbling operation, it is possible for the target's kinetic energy to not monotonically decrease while converging to zero. This is undesirable, as it may result in a prolonged detumbling process. In addition, the evolution of the target's dynamics subjected to an increase in kinetic energy is difficult to predict and may result in larger force errors and even cause the target to rotate out of the workspace of the servicer's manipulator. It is desirable to bring the target's kinetic energy directly to zero during the detumbling procedure. These oscillations are more prominent in targets with a low mass and inertia. To demonstrate this, consider the 
target's kinetic energy expressed about the end-effector as:

$$
\mathrm{K}_{\mathrm{te}}=\frac{1}{2} v_{\mathrm{e}}^{\mathrm{T}} \Lambda_{\mathrm{t}} v_{\mathrm{e}}
$$

If $\dot{\mathrm{K}}_{\mathrm{te}}<0, \forall t>0$, we can conclude that the target's kinetic energy will converge directly to zero.

Evaluating the time derivative of (3.10) requires an expression of the target's external force, $\mathbf{f}_{\mathrm{e}}$, that encompasses the desired force as well as the source of the force error. This is accomplished by utilizing the target's dynamics, $(2.42)$, and (3.7) to express $\mathbf{f}_{\mathrm{e}}$ as:

$$
\mathbf{f}_{\mathrm{e}}=\left(\mathbf{E}_{6}-\mathbf{K}_{\mathrm{fP}}^{-1}\left(\mathbf{K}_{\mathrm{fP}}^{-1}+\Lambda_{\mathrm{t}}\right)^{-1}\right) \mathbf{f}_{\mathrm{eD}}-\mathbf{K}_{\mathrm{fP}}^{-1}\left(\mathbf{K}_{\mathrm{fP}}^{-1}+\Lambda_{\mathrm{t}}\right)^{-1}\left(\Lambda_{\mathrm{t}} \mathbf{J}_{\mathrm{t}} \mathbf{J}_{\mathrm{t}}^{-1} \boldsymbol{v}_{\mathrm{e}}-\mathbf{J}_{\mathrm{t}}^{-\mathrm{T}} \mathbf{c}_{\mathrm{t}}\right)
$$

The time derivative of 3.10 , yields:

$$
\dot{\mathrm{K}}_{\mathrm{te}}=v_{\mathrm{e}}^{\mathrm{T}} \Lambda_{\mathrm{t}} \dot{\boldsymbol{v}}_{\mathrm{e}}+\frac{1}{2} v_{\mathrm{e}}^{\mathrm{T}} \frac{d}{d t}\left(\Lambda_{\mathrm{t}}\right) v_{\mathrm{e}}
$$

The evaluation of (3.12) requires using (3.11). Equation (3.11) can be simplified under the assumption that the target's mass and inertia are sufficiently large so that $\Lambda_{\mathrm{t}}$ is much larger than $\mathbf{K}_{\mathrm{fP}}^{-1}$. This results in the following approximations:

$$
\mathbf{K}_{\mathrm{fP}}^{-1}+\Lambda_{\mathrm{t}} \approx \Lambda_{\mathrm{t}}, \quad \mathbf{E}_{6} \pm \mathbf{K}_{\mathrm{fP}}^{-1} \Lambda_{\mathrm{t}}^{-1} \approx \mathbf{E}_{6}
$$

Note that the off diagonal components of $\Lambda_{\mathrm{t}}^{-1}$ are a function of the target's size and are also scaled by the inverse of the target's inertia: $\mathbf{M}_{\mathrm{t}}^{-1}$. Utilizing the above approximations, 3.13, along with (2.42) and (3.11) allows the first term of (3.12) to be evaluated as follows:

$$
\begin{aligned}
v_{\mathrm{e}}^{\mathrm{T}} \Lambda_{\mathrm{t}} \dot{\boldsymbol{v}}_{\mathrm{e}} & =v_{\mathrm{e}}^{\mathrm{T}} \mathbf{f}_{\mathrm{eD}}-v_{\mathrm{e}}^{\mathrm{T}} \mathbf{J}_{\mathrm{t}}^{-\mathrm{T}} \mathbf{c}_{\mathrm{t}}+v_{\mathrm{e}}^{\mathrm{T}} \Lambda_{\mathrm{t}} \dot{\mathbf{J}}_{\mathrm{t}} \mathbf{J}_{\mathrm{t}}^{-1} \boldsymbol{v}_{\mathrm{e}} \\
& =v_{\mathrm{e}}^{\mathrm{T}} \mathbf{f}_{\mathrm{eD}}-\mathbf{v}_{\mathrm{e}}^{\mathrm{T}} \dot{\mathbf{r}}_{\mathrm{te}}^{\times} \mathbf{w}_{\mathrm{e}} m_{\mathrm{t}}
\end{aligned}
$$

where $\boldsymbol{v}_{\mathrm{e}}^{\mathrm{T}} \mathbf{J}_{\mathrm{t}}^{-\mathrm{T}} \mathbf{c}_{\mathrm{t}}=\boldsymbol{v}_{\mathrm{t}}^{\mathrm{T}} \mathbf{J}_{\mathrm{t}}^{\mathrm{T}} \mathbf{J}_{\mathrm{t}}^{-\mathrm{T}} \mathbf{c}_{\mathrm{t}}=0$ with the use of 2.40), and $\boldsymbol{v}_{\mathrm{e}}^{\mathrm{T}} \Lambda_{\mathrm{t}} \mathbf{J}_{\mathrm{t}} \mathbf{J}_{\mathrm{t}}^{-1} \boldsymbol{v}_{\mathrm{e}}=-\mathbf{v}_{\mathrm{e}}^{\mathrm{T}} \dot{\mathbf{r}}_{\mathrm{te}}^{\times} \mathbf{w}_{\mathrm{e}} m_{\mathrm{t}}$ were utilized in 3.14. 
Utilizing the definition of $\Lambda_{\mathrm{t}}, \mathbf{J}_{\mathrm{t}}$, and $\mathbf{c}_{\mathrm{t}}$ in (2.39), the second term of (3.12) reduces to:

$$
\begin{aligned}
\frac{1}{2} \boldsymbol{v}_{\mathrm{e}}^{\mathrm{T}} \frac{d}{d t}\left(\Lambda_{\mathrm{t}}\right) \boldsymbol{v}_{\mathrm{e}} & =\frac{1}{2} v_{\mathrm{e}}^{\mathrm{T}}\left(\dot{\mathbf{J}}_{\mathrm{t}}^{-\mathrm{T}} \mathbf{M}_{\mathrm{t}} \mathbf{J}_{\mathrm{t}}^{-1}+\mathbf{J}_{\mathrm{t}}^{-\mathrm{T}} \mathbf{M}_{\mathrm{t}} \dot{\mathbf{j}}_{\mathrm{t}}^{-1}+\mathbf{J}_{\mathrm{t}}^{-\mathrm{T}} \dot{\mathbf{M}}_{\mathrm{t}} \mathbf{J}_{\mathrm{t}}^{-1}\right) \boldsymbol{v}_{\mathrm{e}} \\
& =\frac{1}{2}\left(\mathbf{v}_{\mathrm{t}}^{\mathrm{T}} \dot{\mathbf{r}}_{\mathrm{te}}^{\times} \mathbf{w}_{\mathrm{e}} m_{\mathrm{t}}-\mathbf{w}_{\mathrm{e}}^{\mathrm{T}} \dot{\mathbf{r}}_{\mathrm{te}}^{\times} \mathbf{v}_{\mathrm{e}} m_{\mathrm{t}}\right) \\
& =\mathbf{v}_{\mathrm{e}}^{\mathrm{T}} \dot{\mathbf{r}}_{\mathrm{te}}^{\times} \mathbf{w}_{\mathrm{e}} m_{\mathrm{t}}
\end{aligned}
$$

where $\mathbf{J}_{\mathrm{t}}^{-\mathrm{T}} \dot{\mathbf{M}}_{\mathrm{t}} \mathbf{J}_{\mathrm{t}}^{-1}=\dot{\mathbf{M}}_{\mathrm{t}}$ and $\boldsymbol{v}_{\mathrm{e}}^{\mathrm{T}} \dot{\mathbf{M}}_{\mathrm{t}} \boldsymbol{v}_{\mathrm{e}}=0$.

Combining (3.14) and 3.15) in (3.12) results in:

$$
\dot{\mathrm{K}}_{\mathrm{te}}=\boldsymbol{v}_{\mathrm{e}}^{\mathrm{T}} \mathbf{f}_{\mathrm{eD}}=-\left\|\mathbf{v}_{\mathrm{e}}\right\| \mathbf{F}_{\max }-\left\|\mathbf{w}_{\mathrm{e}}\right\| \tau_{\max }<0
$$

which shows that the target's kinetic energy goes directly to zero if the assumptions in (3.13) are satisfied. If either the target's mass or inertia tensor are small relative to $\mathbf{K}_{\mathrm{fP}}^{-1}$, the above analysis becomes invalid as assumption (3.13) cannot be applied and there will be the potential for an exchange of kinetic energy between the target and servicer. To demonstrate this, we evaluate $\dot{\mathrm{K}}_{\mathrm{te}}$ without applying assumption 3.13 . The resultant equation can be expressed as:

$$
\dot{\mathrm{K}}_{\mathrm{te}}=\alpha+\beta
$$

where $\alpha=-\left\|\mathbf{v}_{\mathrm{e}}\right\| \mathbf{F}_{\max }-\left\|\mathbf{w}_{\mathrm{e}}\right\| \tau_{\max }$ and $\beta=\boldsymbol{v}_{\mathrm{t}}^{\mathrm{T}} \Gamma\left(-\mathbf{f}_{\mathrm{eD}}+\mathbf{J}_{\mathrm{t}}^{-\mathrm{T}} \mathbf{c}_{\mathrm{t}}-\Lambda_{\mathrm{t}} \mathbf{J}_{\mathrm{t}} \mathbf{J}_{\mathrm{t}}^{-1} \boldsymbol{v}_{\mathrm{e}}\right)$ with $\Gamma=\mathbf{K}_{\mathrm{fP}}^{-1}\left(\mathbf{K}_{\mathrm{fP}}^{-1}+\right.$ $\left.\Lambda_{\mathrm{t}}\right)^{-1}$.

The additional term, $\beta$, aries because of the neglect of assumption (3.13) and is a function of not only the target's inertia but of its linear and angular velocity. Hence, the conclusion drawn from (3.16) may be inaccurate for a target whose inertia is large enough to satisfy assumption (3.13) if its motion is sufficiently large to the cause the presence of $\beta$ in 3.17). Thus, it is of interest to determine the target's characteristics that ensure $\dot{\mathrm{K}}_{\mathrm{te}}<0, \forall t>0$.

Upper bounds can be placed on $\alpha$ and $\beta$ as follows:

$$
\begin{gathered}
\alpha \leq-K\left\|v_{\mathrm{e}}\right\| \\
\beta \leq\left\|v_{\mathrm{e}}\right\|\|\Gamma\|\left(\left\|\mathbf{f}_{\mathrm{eD}}\right\|+\|\gamma\|\right)
\end{gathered}
$$


where $K=\min \left\{\mathrm{F}_{\max }, \tau_{\max }\right\},\left\|\mathbf{f}_{\mathrm{eD}}\right\|=\left(\mathrm{F}_{\max }^{2}+\tau_{\max }^{2}\right)^{\frac{1}{2}}$ and $\|\gamma\|=\left\|\mathbf{J}_{\mathrm{t}}^{-\mathrm{T}} \mathbf{c}_{\mathrm{t}}-\Lambda_{\mathrm{t}} \mathbf{J}_{\mathrm{t}} \mathbf{J}_{\mathrm{t}}^{-1} \boldsymbol{v}_{\mathrm{e}}\right\|$. Utilizing the definition of $\Lambda_{\mathrm{t}}, \mathbf{J}_{\mathrm{t}}$ and $\mathbf{c}_{\mathrm{t}}$ in (2.39), the evaluation of $\|\gamma\|$ in (3.19) is as follows:

$$
\begin{aligned}
\gamma & =\left(\mathbf{J}_{\mathrm{t}}^{-\mathrm{T}} \mathbf{c}_{\mathrm{t}}-\Lambda_{\mathrm{t}} \mathbf{J}_{\mathrm{t}} \mathbf{J}_{\mathrm{t}}^{-1} \boldsymbol{v}_{\mathrm{e}}\right) \\
& =\left[\begin{array}{c}
m_{\mathrm{t}} \dot{\mathbf{r}}_{\mathrm{te}}^{\times} \mathbf{w}_{\mathrm{t}} \\
\mathbf{w}_{\mathrm{t}} \times \mathbf{I}_{\mathrm{t}} \mathbf{w}_{\mathrm{t}}-m_{\mathrm{t}} \mathbf{r}_{\mathrm{te}}^{\times} \dot{\mathbf{r}}_{\mathrm{te}}^{\times} \mathbf{w}_{\mathrm{t}}
\end{array}\right] .
\end{aligned}
$$

Utilizing Lemma 1 in [77], the components of 3.20 can be upper bounded as follows: $\left\|m_{\mathrm{t}} \dot{\mathbf{r}}_{\mathrm{te}}^{\times} \mathbf{w}_{\mathrm{t}}\right\| \leq m_{\mathrm{t}}\left\|\mathbf{r}_{\mathrm{te}}\right\|\left\|\mathbf{w}_{\mathrm{t}}\right\|^{2}, \quad\left\|\mathbf{w}_{\mathrm{t}} \times \mathbf{I}_{\mathrm{t}} \mathbf{w}_{\mathrm{t}}\right\| \leq\left[\lambda_{\max }^{2}\left(\mathbf{I}_{\mathrm{t}}\right)-\lambda_{\min }^{2}\left(\mathbf{I}_{\mathrm{t}}\right)\right]^{\frac{1}{2}}\left\|\mathbf{w}_{\mathrm{t}}\right\|^{2}$ and $\left\|m_{\mathrm{t}} \mathbf{r}_{\mathrm{te}}^{\times} \dot{\mathbf{r}}_{\mathrm{te}}^{\times} \mathbf{w}_{\mathrm{t}}\right\| \leq m_{\mathrm{t}}\left\|\mathbf{r}_{\mathrm{te}}\right\|^{2}\left\|\mathbf{w}_{\mathrm{t}}\right\|^{2}$. Utilizing these bounds and noting that $\left\|\mathbf{w}_{\mathrm{t}}\right\|^{2} \leq\left\|\boldsymbol{v}_{\mathrm{e}}\right\|^{2}$, the magnitude of $\gamma$ is upper bounded as:

$$
\|\gamma\| \leq h\left\|\boldsymbol{v}_{\mathrm{e}}\right\|^{2}, \quad h=\left[\left(m_{\mathrm{t}}\left\|\mathbf{r}_{\mathrm{te}}\right\|\right)^{2}+\left(\left[\lambda_{\max }^{2}\left(\mathbf{I}_{\mathrm{t}}\right)-\lambda_{\min }^{2}\left(\mathbf{I}_{\mathrm{t}}\right)\right]^{\frac{1}{2}}+m_{\mathrm{t}}\left\|\mathbf{r}_{\mathrm{te}}\right\|^{2}\right)^{2}\right]^{\frac{1}{2}} .
$$

Utilizing the lower bound on $\mathrm{K}_{\mathrm{te}}, \frac{1}{2} \lambda_{\min }\left(\Lambda_{\mathrm{t}}\right)\left\|\boldsymbol{v}_{\mathrm{e}}\right\|^{2} \leq \mathrm{K}_{\mathrm{te}}$, and 3.21 , results in the following upper bound on $\beta$ :

$$
\beta \leq\left\|v_{\mathrm{e}}\right\|\|\Gamma\|\left(\left\|\mathbf{f}_{\mathrm{eD}}\right\|+\frac{2 h \mathrm{~K}_{\mathrm{te}}}{\lambda_{\min }\left(\Lambda_{\mathrm{t}}\right)}\right) .
$$

Combining (3.22) and (3.18) in (3.17) results in:

$$
\dot{\mathrm{K}}_{\mathrm{te}} \leq-\left\|v_{\mathrm{e}}\right\|\left(\gamma_{2}-\frac{2\|\Gamma\| h \mathrm{~K}_{\mathrm{te}}}{\lambda_{\min }\left(\Lambda_{\mathrm{t}}\right)}\right), \quad \gamma_{2}=K-\|\Gamma\|\left\|\mathbf{f}_{\mathrm{eD}}\right\| .
$$

From (3.23), provided $\gamma_{2}>0$, for $\dot{\mathrm{K}}_{\mathrm{te}}<0$, it is required that:

$$
\left(\gamma_{2}-\frac{2\|\Gamma\| h \mathrm{~K}_{\mathrm{te}}}{\lambda_{\min }\left(\Lambda_{\mathrm{t}}\right)}\right)>0 \rightarrow \mathrm{K}_{\mathrm{te}}<\frac{\lambda_{\min }\left(\Lambda_{\mathrm{t}}\right) \gamma_{2}}{2\|\Gamma\| h}
$$

Additionally, utilizing $\mathrm{K}_{\mathrm{te}} \leq \frac{1}{2} \lambda_{\max }\left(\Lambda_{\mathrm{t}}\right)\left\|\boldsymbol{v}_{\mathrm{e}}\right\|^{2}$ and $\sqrt{3.24}$ results in an estimate of the domain of attraction:

$$
S=\left\{v_{\mathrm{e}} \mid\left\|v_{\mathrm{e}}\right\|<\left(\frac{\lambda_{\min }\left(\Lambda_{\mathrm{t}}\right) \gamma_{2}}{\lambda_{\max }\left(\Lambda_{\mathrm{t}}\right)\|\Gamma\| h}\right)^{\frac{1}{2}}\right\} .
$$

Provided that $\gamma_{2}>0$, it follows that if the target's initial velocity after grasping is contained in the set $S$, then the target's kinetic energy will converge directly to zero since $\dot{\mathrm{K}}_{\mathrm{te}}<0$ in $S$. Furthermore, detumbling the target can be achieved in finite time. Utilizing the upper bounds 
on $\mathrm{K}_{\mathrm{te}}$ and $\left\|v_{\mathrm{e}}\right\|$ from (3.24) and (3.25) in 3.23), results in the following upper bound on the time taken to detumble the target, $t_{\mathrm{r}}$, as:

$$
t_{\mathrm{r}} \leq \frac{\ln \left(1 /\left(1-\xi_{c}\right)\right)}{\zeta}
$$

where $\varsigma=\left[\left(4 \gamma_{2}\|\Gamma\| h\right) /\left(\lambda_{\min }\left(\Lambda_{\mathrm{t}}\right) \lambda_{\max }\left(\Lambda_{\mathrm{t}}\right)\right)\right]^{1 / 2}$ and $0 \leq \xi_{c}<1$ such that $\mathrm{K}_{\mathrm{te}}\left(t_{o}\right)=\xi_{c} \mathrm{~K}_{\mathrm{te}}^{\mathrm{UP}}$ where $\mathrm{K}_{\mathrm{te}}^{\mathrm{UP}}$ is the upper bound on $\mathrm{K}_{\mathrm{te}}$ defined in 3.24 .

The two requirements, $\gamma_{2}>0$ and $v_{\mathrm{e}} \in S$, that arise from the above analysis imposes constraints on the target's inertial parameters in order for $\dot{\mathrm{K}}_{\mathrm{te}}<0, \forall t>0$. The first constraint, $\gamma_{2}>0$ in 3.23 , is dependent on $K,\left\|\mathbf{f}_{\mathrm{eD}}\right\|, \mathbf{K}_{\mathrm{fP}}$ and $\|\Gamma\|$. For a fixed $K,\left\|\mathbf{f}_{\mathrm{eD}}\right\|$ and $\mathbf{K}_{\mathrm{fP}},\|\Gamma\|$ is inversely proportional to the inertia of the target $\left(\left\|\mathbf{M}_{t}\right\|\right)$ and hence $\gamma_{2}$ will approach 0 as the target's inertia decreases. The evaluation of the second constraint is dependent on the grasping technique utilized in the pre-grasping phase as this will determine the initial velocity of the post-grasping phase. The target's velocity in the pre-grasping phase can be estimated from vision based techniques [8]. If the approach utilized in the pre-grasping phase can capture the target with little to no impact as in [8], then the estimated velocity prior to grasping can be used to evaluate if the velocity of the grasping surface, $v_{\mathrm{e}}$, after capture (start of post-grasping phase) is contained in the set $S$. Using estimated bounds on the target's inertial parameters, the above constraints can be evaluated to determine if the target can be detumbled under the condition that $\dot{\mathrm{K}}_{\mathrm{te}}<0$. Furthermore, increasing the proportional gain, $\mathbf{K}_{\mathrm{fP}}$, allows for greater uncertainty in bounds on the target's inertial parameters (i.e., an increase in $\mathbf{K}_{\mathrm{fP}}$ ensures that $\gamma_{2}>0$ for targets with smaller and smaller inertia).

\subsubsection{Force/Torque Error}

Rearranging 3.11 , the force/torque error, $\tilde{\mathbf{f}}_{\mathrm{e}}=\mathbf{f}_{\mathrm{eD}}-\mathbf{f}_{\mathrm{e}}$, results in:

$$
\tilde{\mathbf{f}}_{\mathrm{e}}=\Gamma\left[\mathbf{f}_{\mathrm{eD}}+\Lambda_{\mathrm{t}} \dot{\mathbf{J}}_{\mathrm{t}} \mathbf{J}_{\mathrm{t}}^{-\mathrm{T}} \boldsymbol{v}_{\mathrm{e}}-\mathbf{J}_{\mathrm{t}}^{-\mathrm{T}} \mathbf{c}_{\mathrm{t}}\right]
$$

Targets that satisfy $\gamma_{2}>0\left(\gamma_{2}\right.$ defined in 3.23) $)$ and (3.25) ensures that the target's kinetic energy monotonically converges to zero and hence the terms on the right side of (3.27) are bounded and will converge to zero. Hence, the force/torque error can be upper bounded by 
utilizing (3.21) and 3.25 , resulting in:

$$
\left\|\Gamma\left[\mathbf{f}_{\mathrm{eD}}+\Lambda_{\mathrm{t}} \dot{\mathbf{J}}_{\mathrm{t}} \mathbf{J}_{\mathrm{t}}^{-\mathrm{T}} \boldsymbol{v}_{\mathrm{e}}-\mathbf{J}_{\mathrm{t}}^{-\mathrm{T}} \mathbf{c}_{\mathrm{t}}\right]\right\|<G
$$

where $G=\|\Gamma\|\left\|\mathbf{f}_{\mathrm{eD}}\right\|+\lambda_{\min }\left(\Lambda_{\mathrm{t}}\right) \gamma_{2} / \lambda_{\max }\left(\Lambda_{\mathrm{t}}\right)$. Furthermore, considering the definition of $\Gamma$ presented after (3.17), the force/torque error can be reduced by an increase in $\mathbf{K}_{\mathrm{fP}}$, and is lower bounded by the second term of $G$.

\subsubsection{Numerical Simulation}

This subsection presents the evaluation of the proposed detumbling controller to detumble a target in the post grasping phase. The manipulator and base attitude control inputs, $\tau_{\mathrm{m}}$ and $\tau_{\mathrm{b}}$, are defined as per (3.1). The auxiliary inputs, $\mathbf{u}_{\mathrm{f}}$ and $\mathbf{u}_{\mathrm{b}}$, to detumble the target and to reject the target's momentum are defined as per (3.6) and (3.3a), respectively. Furthermore, reference end-effector force/torque, $\left(\mathbf{F}_{\mathrm{eD}}\right)$ and $\left(\tau_{\mathrm{eD}}\right)$, are modified in order to avoid chattering as the end-effector's linear and angular velocities converge to zero during the detumbling of the target. As the target's kinetic energy converges to zero, both the end-effector's linear and angular velocity will converge at different rates. This presents a drawback due to the kinematic coupling between the end-effector's linear and angular velocities and that of the target: the endeffector's linear and angular velocity will not remain at zero unless both are zero. As a result, chattering of the desired force/torque in (3.4) and (3.5) will occur. To avoid this, the desired force $\left(\mathbf{F}_{\mathrm{eD}}\right)$ and torque $\left(\tau_{\mathrm{eD}}\right)$ are multiplied by $s_{\mathrm{v}}$ and $s_{\mathrm{w}}$, respectively, where $s_{(\cdot)}=\frac{\|(\cdot)\|}{\|(\cdot)\|+\varepsilon_{(\cdot)}}$. This results in the following:

$$
\begin{aligned}
& \mathbf{F}_{\mathrm{eD}}=-\left(\frac{\mathbf{v}_{\mathrm{e}}}{\left\|\mathbf{v}_{\mathrm{e}}\right\|+\varepsilon_{v}}\right) \mathrm{F}_{\max } \\
& \tau_{\mathrm{eD}}=-\left(\frac{\mathbf{w}_{\mathrm{e}}}{\left\|\mathbf{w}_{\mathrm{e}}\right\|+\varepsilon_{w}}\right) \tau_{\max }
\end{aligned}
$$

where $\varepsilon_{v}$ and $\varepsilon_{w}$ are positive constants used to attenuate the magnitude of the desired force and torque, respectively, as the end-effector's linear and angular velocities converge to zero.

Numerical simulations are carried out using a MATLAB Simulink add-on, SimMechanics [1], emulating a 7-degree-of-freedom (DOF) space manipulator servicer satellite that is based on a modified model of ETS-VII system. The model parameters of the servicer are described 
in Appendix A.

As the servicer's manipulator has 7-DOF, it will have redundancy with respect to the detumbling task. The detumbling of the target will result in its momentum being transferred to the manipulator as well as the servicer's base. Redundant manipulators will have momentum (joint motion) in the null space of the detumbling task when the target is brought to rest. This momentum will be rejected by the base attitude control system. However, this process can be slow as momentum is only rejected by the base attitude control system if the manipulator null space motion disturbs the base. The transfer of momentum to the servicer's base can be improved with the use of joint damping in the null space of the detumbling task. This is similar to reaction null space damping in [42], but the null space is formulated with respect to the detumbling task in operational space. The manipulator joint torque to achieve this is defined as:

$$
\tau_{\mathrm{ND}}=-\mathbf{M}\left[\begin{array}{c}
\mathbf{P}_{\mathrm{ND}} \mathbf{K}_{\mathrm{ND}} \dot{\phi}_{\mathrm{m}} \\
\mathbf{0}
\end{array}\right]
$$

where $\mathbf{P}_{\mathrm{ND}} \in \mathbb{R}^{m \times m}$ stands for the projection operator onto the null space of $\mathbf{A}_{\mathrm{m}} \in \mathbb{R}^{6 \times m}$ which in turn maps the manipulator's joint velocities from joint space to task space after eliminating the base's linear velocity ( $\mathbf{A}_{\mathrm{m}}$ is defined in 2.31$)$ ). The gain, $\mathbf{K}_{\mathrm{ND}} \in \mathbb{R}^{m \times m}$ defines the damping gain matrix, and $\mathbf{P}_{\mathrm{ND}}=\left(\mathbf{E}_{m}-\mathbf{A}_{\mathrm{m}}^{+} \mathbf{A}_{\mathrm{m}}\right)$ where $(\cdot)^{+}$denotes the Moor-Penrose pseudoinverse of a matrix. The resulting detumbling controller is formulated by adding (3.31) to (3.1). For the numerical simulation study, $\mathbf{K}_{\mathrm{ND}}=\mathbf{E}_{7}$.

The target is grasped at $t=0 \mathrm{sec}$ and is rigidly attached to the last link. As a result of impact, the target's initial angular momentum is redistributed in the servicer-target system, causing a change in the servicer-target system velocities. The velocities of the servicer-target system after grasping $\left(t^{+}=0 \mathrm{sec}\right)$ are computed as in [67]. Functions from the SpaceDyn toolbox presented in [69] were utilized to compute the servicer's mass matrix, Jacobian and nonlinear forces.

The target is assumed to be a cube $(1.95 \mathrm{~m} \times 1.95 \mathrm{~m} \times 1.95 \mathrm{~m}$ cube $)$ with the following properties: $\mathbf{I}_{\mathrm{t}}=\operatorname{diag}([212.8,212.8,219.9]) \mathrm{kgm}^{2}, m_{\mathrm{t}}=350 \mathrm{~kg}$ and an initial angular velocity of $\mathbf{w}_{\mathrm{t}}=[-3.9,-3.9,-6.5]^{T} \mathrm{deg} / \mathrm{s}$. The end-effector's force/torque magnitude $\mathrm{F}_{\max }$, and $\tau_{\max }$ are set at $8 \mathrm{~N}$ and $10 \mathrm{Nm}$, respectively. $\varepsilon_{v}$ and $\varepsilon_{w}$ are set at $10^{-2} \mathrm{~m} / \mathrm{s}$ and $10^{-2} \mathrm{deg} / \mathrm{s}$, respectively. The servicer's attitude and force controller utilize the following gains: $\mathbf{K}_{\mathrm{AD}}=1.5 \mathbf{E}_{3}$ and $\mathbf{K}_{\mathrm{fP}}=$ $0.2 \mathbf{E}_{6}$. 

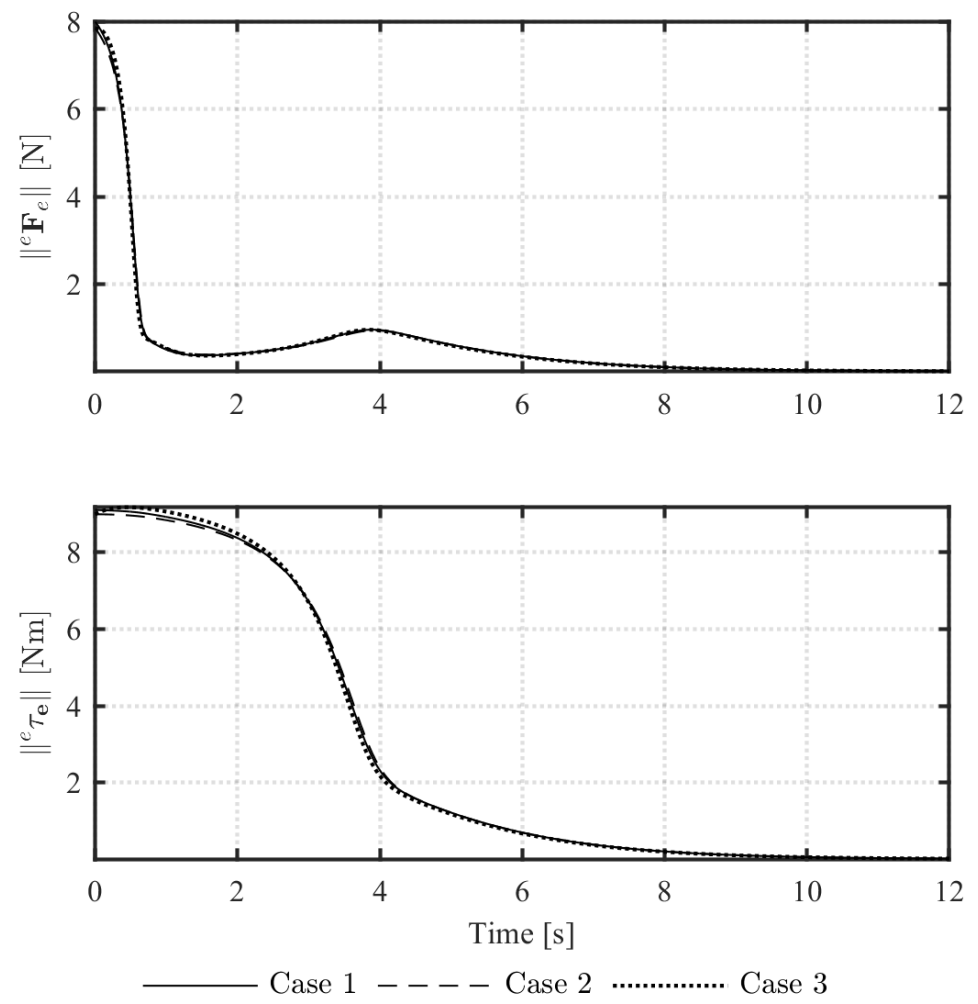

Figure 3.1: End-effector's force and torque profile for Cases 1, 2 and 3.

The evaluation is carried out for three different cases. The first case is carried out utilizing the above mentioned controller gains. The second case, introduces servicer model uncertainty to the Case 1. The third case introduces an integral controller as in [64] to $\mathbf{u}_{\mathrm{f}}$ in (3.6) to Case 2. The integral controller is defined as follows: $\mathbf{K}_{\mathrm{fI}} \int\left(\mathbf{f}_{\mathrm{eD}}-\mathbf{f}_{\mathrm{e}}\right) d t$ with $\mathbf{K}_{\mathrm{fI}}=0.6 \mathbf{E}_{6}$. Servicer model uncertainty is introduced by modeling the servicer's geometry and inertia as $90 \%$ of their true values, and the detumbling controller drops compensation of the nonlinear term, $\overline{\mathbf{c}}$, in 3.1. As per the stated assumptions in Chapter 2, measurements of the servicer system are assumed to be obtained at a sample rate of $0.01 \mathrm{~s}$ from an inertial observer as suggested in [49].

The end-effector force/torque profile and force/torque tracking error are presented in Figs. 3.1, 3.2 and 3.3. For Cases 1 through 3, the end-effector force/torque are within the force/torque limits, $\mathrm{F}_{\max }=10 \mathrm{~N}$ and $\tau_{\max }=10 \mathrm{Nm}$, for the duration of the detumbling period (Fig. 3.1). The left plots of Figs. 3.2 and 3.3 indicate that the force and torque error are bounded by the force and torque error bound derived in Section 3.3.2. (3.28). The force/torque profiles for Cases 1 through 3 appear to be identical in Fig. 3.1. However, subtle difference are observed when the 

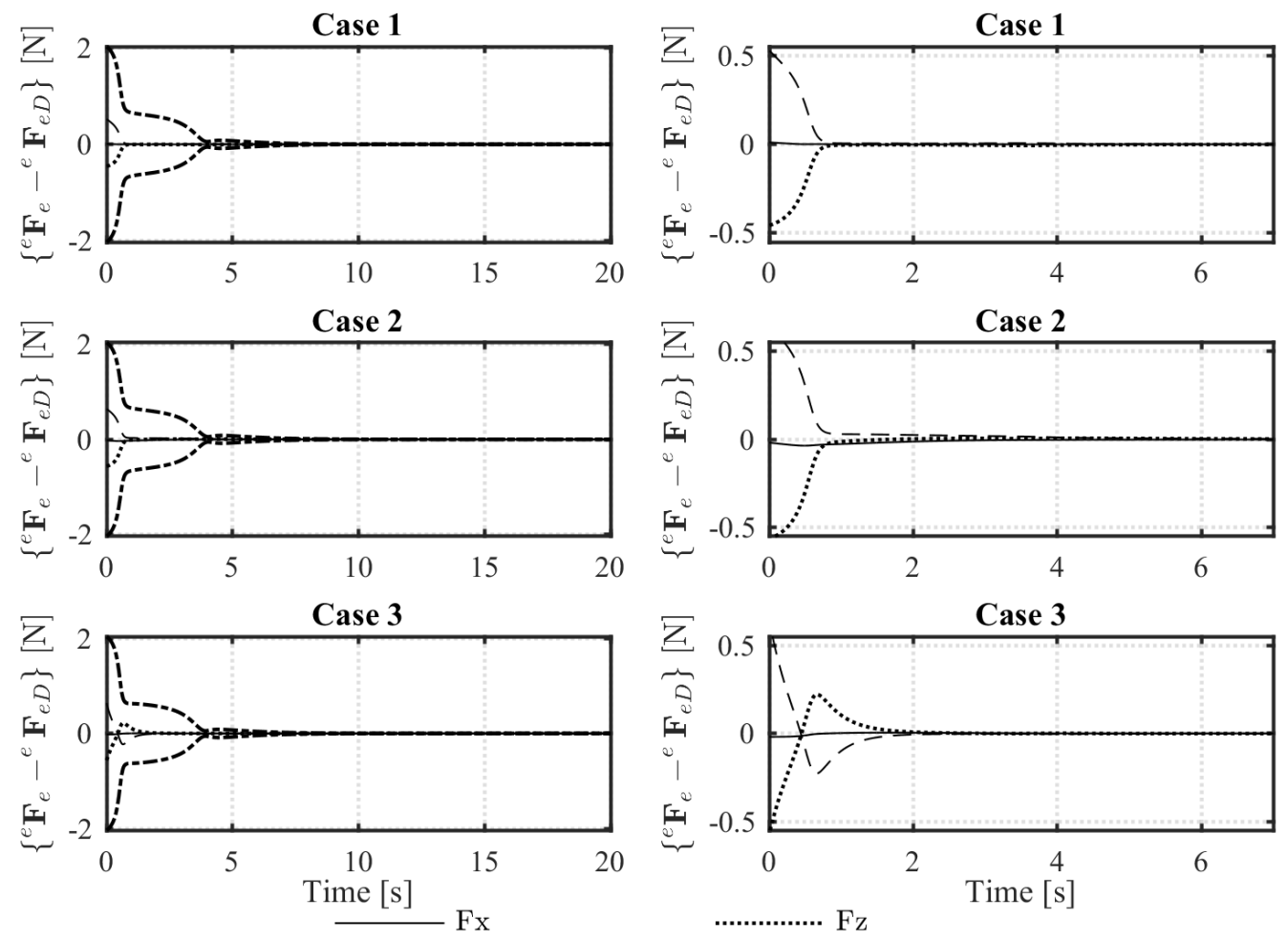

$----F y$

-----.. Force Error Bound

Figure 3.2: End-effector's force error profile. Left plots depict force error bound that is computed as per (3.28). Right plots show enlarged force error.

force and torque tracking error are enlarged (right plots of Figs. 3.2 and 3.3). Force and torque tracking errors are larger in Case 2 than in Case 1 due to servicer model uncertainty in Case 2. The introduction of the integral gain, $\mathbf{K}_{\mathrm{ff}}$, in Case 3 allows for better convergence of the force and torque error but at the expense of larger peak force/torque error (Case 3, right plots in Figs. 3.2 and 3.3.

Detumbling of the target is considered complete once it is brought to rest. This is demonstrated in Figs. 3.4 and 3.5 as the end-effector's linear and angular velocity profiles are presented in Fig. 3.4 and the angular momentum distribution of the servicer-target system is presented in Fig. 3.5. In Fig. 3.4, the end-effector's linear and angular velocity components converge to zero and are similar for Cases 1 through 3. In Fig. 3.5, the target's momentum is rejected as it converges to zero for Cases 1 through 3. In addition, Fig. 3.5 also demonstrates the effect of null space damping for a redundant manipulator. A lower value of $\mathbf{K}_{N D}$ 

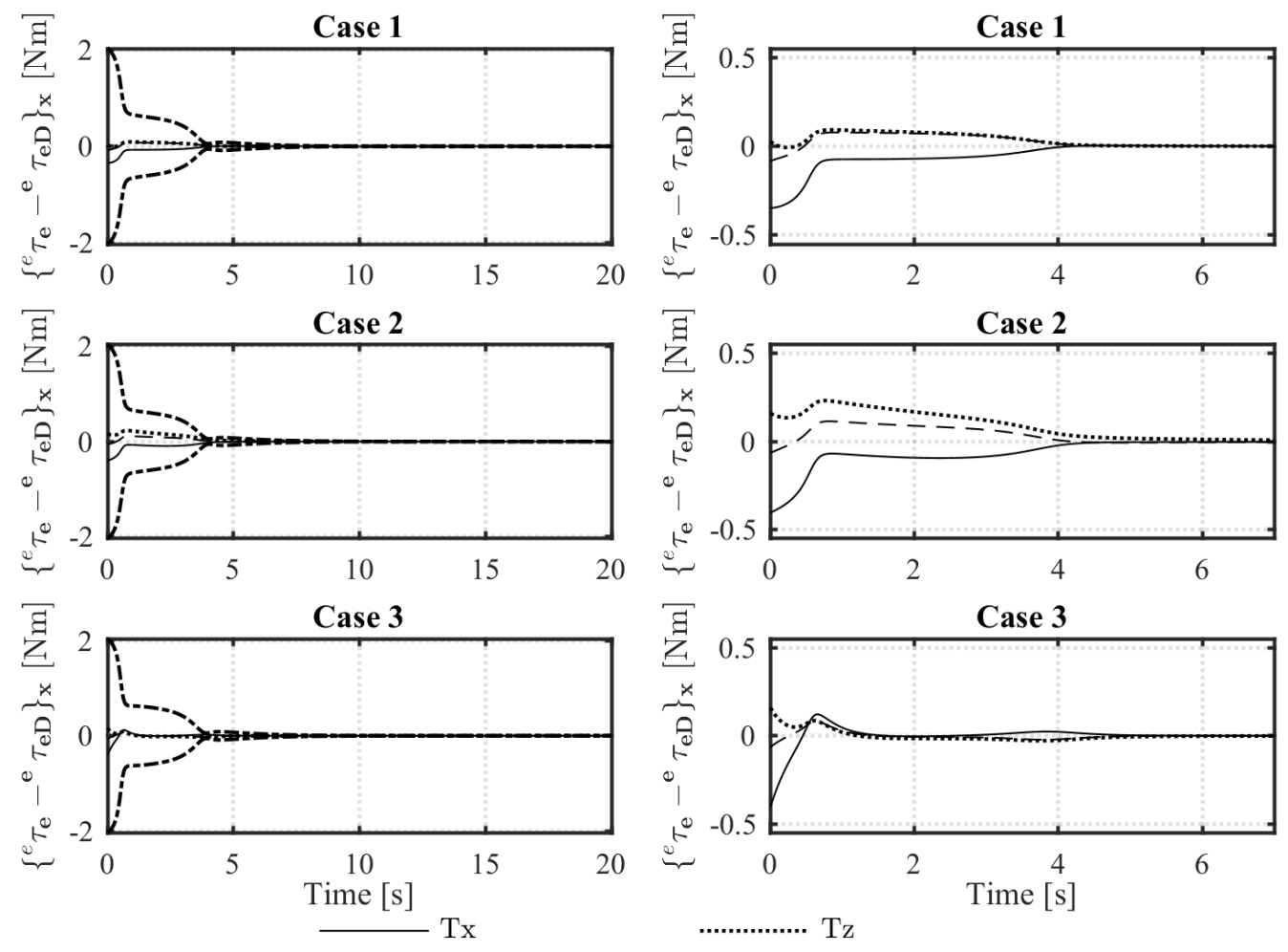

Figure 3.3: End-effector's torque error profile. Left plots depict torque error bound that is computed as per (3.28). Right plots show enlarged torque error.

will prolong the detumbling procedure as the manipulator's momentum in the null space of the detumbling task will take time to transfer to the servicer's base where it will be rejected. As per the stability analysis presented earlier, the kinetic energy of the target, (3.10), is presented in Fig. 3.6 and is shown to monotonically converge to zero for Cases 1 through 3.

As indicated from Figs. 3.1 through 3.6, detumbling is achieved for all three cases while respecting force/torque limits without the use of the target's inertial parameters. Determining if the target could be detumbled under the condition that $\dot{\mathrm{K}}_{\mathrm{te}}<0$ is determined based on prelaunched data of the satellite as discussed at the end of Section 3.3.1. Furthermore, in the above analysis, the rotation rate $\left(\mathbf{w}_{t}\right)$ utilized for the target initial angular velocity may appear considerably low. However, a low tumbling rate is reasonable as the motion rate of most targets can be characterized as a slow rotation about a major axis with a small nutation angle [53]. For example, ground based observation of ADEOS 1 described its attitude motion as composed 
of two rotational components: $0.1 \mathrm{deg} / \mathrm{s}$ about the satellite's main body and another about the satellite's boom of about $0.4 \mathrm{deg} / \mathrm{s}$ [77]. Observation of Envisat resulted in an average rotation rate of $3.5 \mathrm{deg} / \mathrm{s}$ [62].
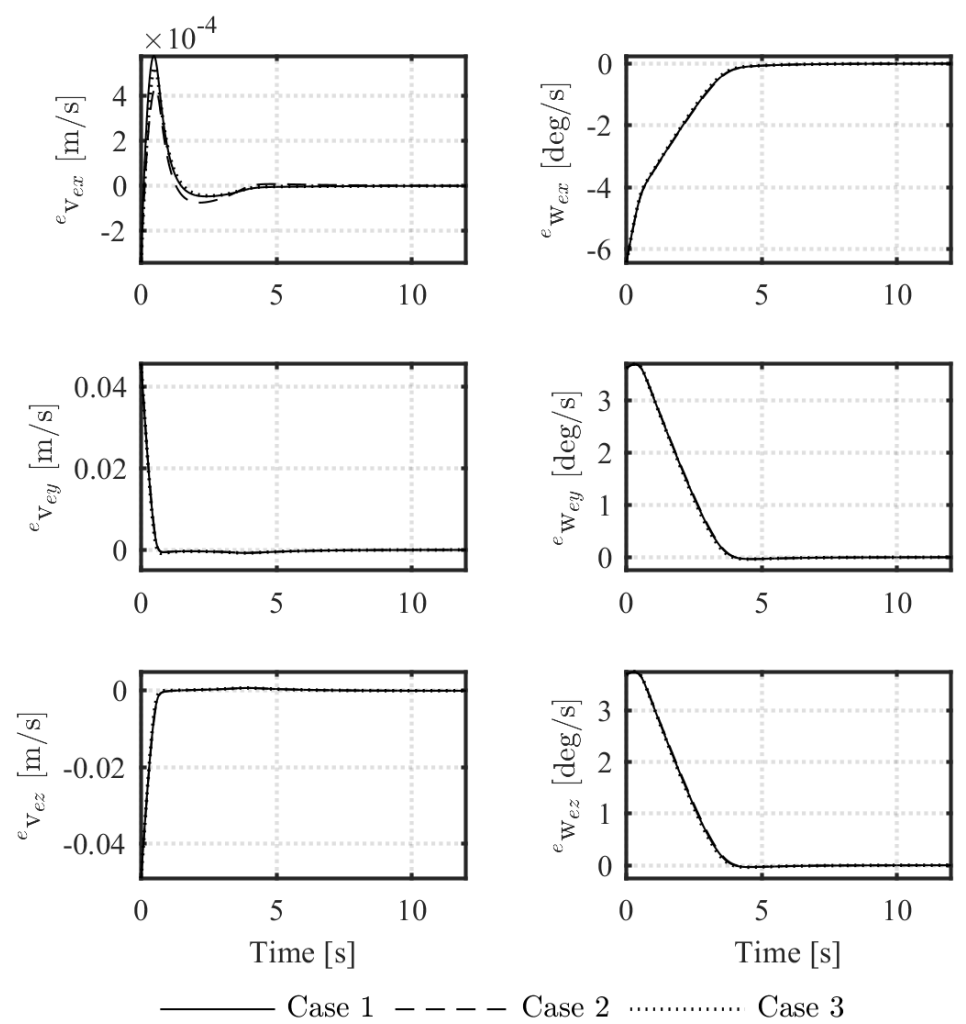

Figure 3.4: End-effector's linear and angular velocities for Cases 1, 2 and 3. 

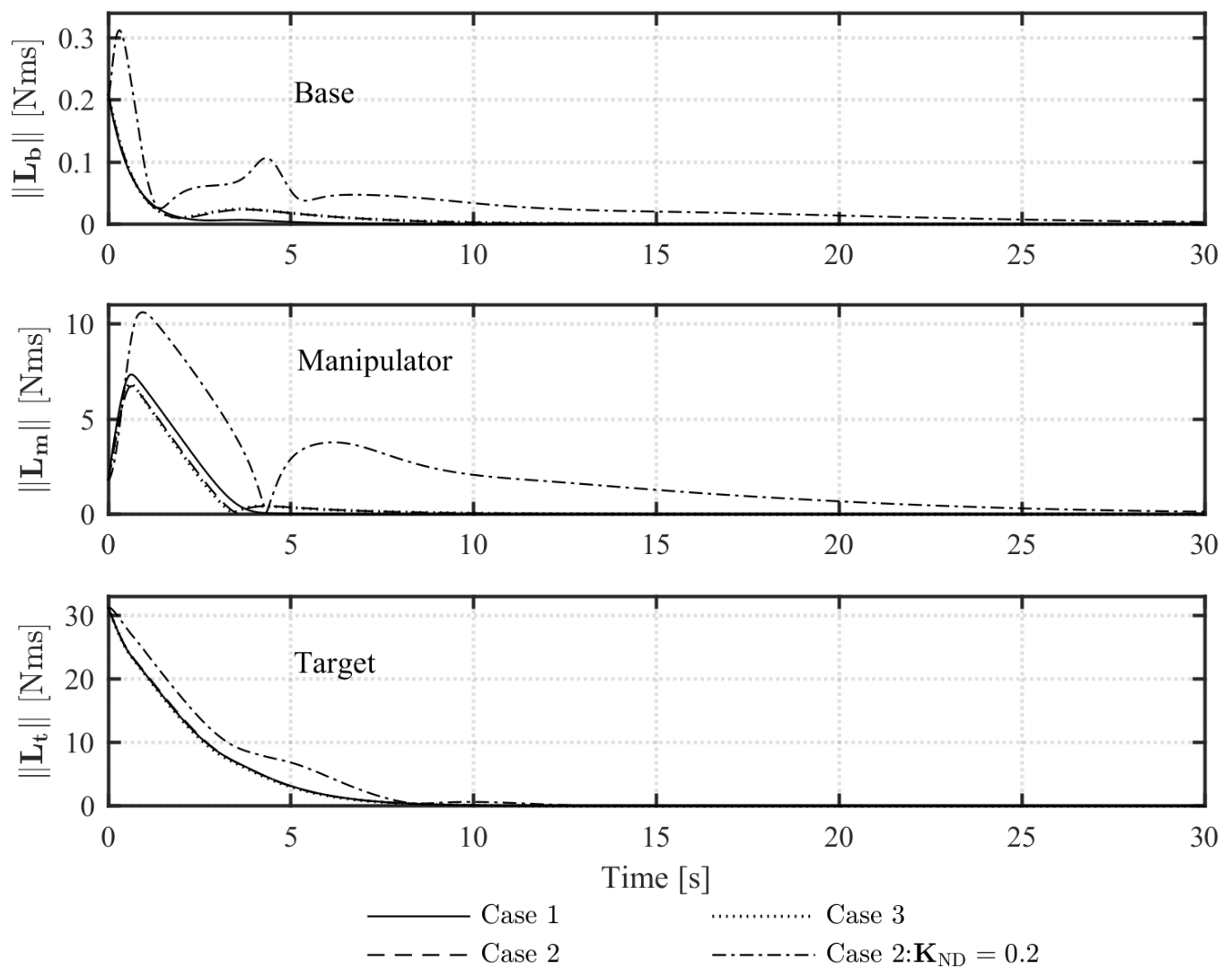

Figure 3.5: Angular momentum distribution for Cases 1, 2 and 3.

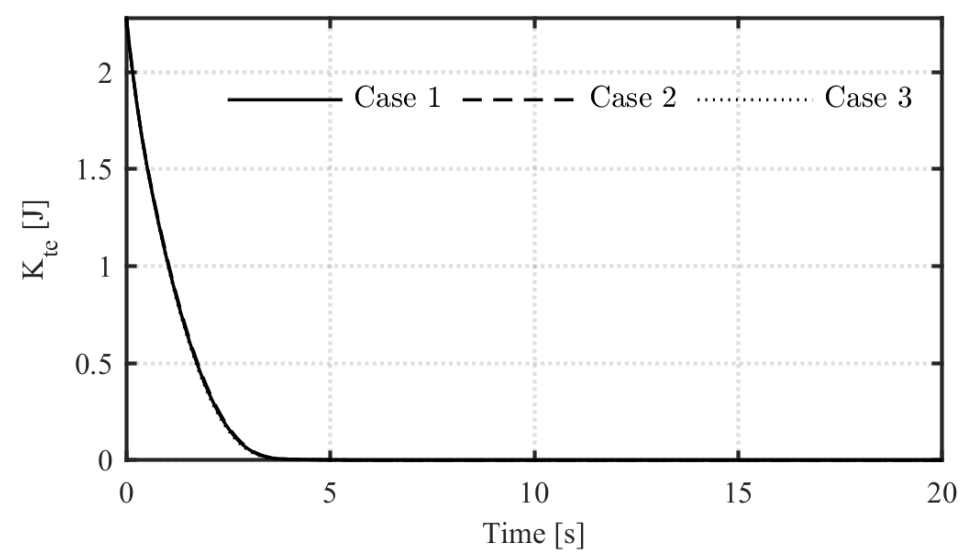

Figure 3.6: Target's kinetic energy for Cases 1, 2 and 3. 


\subsection{Robust Detumbling of Target}

In this section, the design of $\mathbf{u}_{\mathrm{f}}$ is extended with the addition of a continuous robust compensator that is designed to compensate for changes in the servicer's inertial parameters due to the grasped target at the servicer's end-effector in the post-grasping phase. In the previous section, detumbling of the target is seen to be possible without the addition of any robust compensator and the resultant detumbling controller is simple. However, implementation of the detumbling controller requires one to determine if the potential target slated for servicing will satisfy the detumbling criteria: $\gamma_{2}$ defined in (3.23) is greater than zero, and $v_{\mathrm{e}} \in S$ with $S$ defined in (3.25). Bounds on the target's inertial parameters can be utilized to evaluate the detumbling criteria. However, the detumbling criteria may not be possible to satisfy if the estimated bounds on the target's inertial parameters are conservative (i.e., the detumbling criteria becomes impractical to satisfy as the set $S$ may become small).

Alternatively, in this section, instead of imposing restrictions in the form of the detumbling criteria (i.e., $\gamma_{2}>0$ and $v_{\mathrm{e}} \in S$ ), a robust compensator is designed to compensate for changes in the servicer's inertial parameters as a result of the captured target during the post-grasping phase. The continuous robust compensator is designed in this section and its gains are presented to be determined as a function of bounds on the target's inertial parameters. Following this, an adaptive technique is utilized to allow the estimation of bounds on the target's inertial parameters in real-time. This eliminates the use of conservative estimates of bounds on the target's inertial parameters in the robust compensator. Furthermore, the desired force/torque in (3.4) and (3.5) are modified and stability proof of the closed-loop system is presented.

Prior to presenting the robust detumbling controller, the target's equation of motion are presented once more, but are expressed in a body fixed frame on the target. This is done so that the time derivative of the target's kinetic energy does not involve the time derivative of the target's mass matrix.

\subsubsection{Target Dynamics in Body Fixed Frame}

From Fig. 2.1, two body fixed frames are denoted as $\Sigma_{\mathrm{t}}$ and $\Sigma_{\mathrm{e}} . \Sigma_{\mathrm{t}}$ is located at the target's center of mass and $\Sigma_{\mathrm{e}}$ is located at the grasping point. As per assumption 2, the target is assumed to be rigidly grasped. Hence, the body fixed frame at the target's grasping point, $\Sigma_{\mathrm{e}}$, also represents the end-effector's frame. 
The orientation of $\Sigma_{\mathrm{e}}$ is known and $\Sigma_{\mathrm{t}}$ is assumed to have the same orientation as $\Sigma_{\mathrm{e}}$, resulting in ${ }^{\mathrm{e}} \mathbf{R}_{\mathrm{t}}=\mathbf{E}_{3}$. Let ${ }^{\mathrm{t}} \boldsymbol{v}_{\mathrm{t}}=\left[{ }^{\mathrm{t}} \mathbf{v}_{\mathrm{t}}^{\mathrm{T}},{ }^{\mathrm{t}} \mathbf{w}_{\mathrm{t}}^{\mathrm{T}}\right]^{\mathrm{T}} \in \mathbb{R}^{6}$ represent the target's linear and angular velocity about its center of mass in $\Sigma_{\mathrm{t}}$. The target's linear and angular velocity about its center of mass can also be expressed as ${ }^{\mathrm{e}} \boldsymbol{v}_{\mathrm{t}}=\left[{ }^{\mathrm{e}} \mathbf{v}_{\mathrm{t}}^{\mathrm{T}},{ }^{\mathrm{e}} \mathbf{w}_{\mathrm{t}}^{\mathrm{T}}\right]^{\mathrm{T}} \in \mathbb{R}^{6}$ in $\Sigma_{\mathrm{e}}$ where ${ }^{\mathrm{t}} \boldsymbol{v}_{\mathrm{t}}={ }^{\mathrm{e}} \boldsymbol{v}_{\mathrm{t}}$ since ${ }^{\mathrm{e}} \mathbf{R}_{\mathrm{t}}=\mathbf{E}_{3}$. Utilizing the above, the equations of motion of the target are:

$$
\mathbf{M}_{\mathrm{te}}{ }^{\mathrm{e}} \dot{\boldsymbol{v}}_{\mathrm{t}}+\mathbf{c}_{\mathrm{te}}=\mathbf{J}_{\mathrm{te}}^{\mathrm{T}}\left({ }^{\mathrm{e}} \mathbf{f}_{\mathrm{e}}\right)
$$

where $\mathbf{M}_{\mathrm{te}} \in \mathbb{R}^{6 \times 6}, \mathbf{c}_{\mathrm{te}} \in \mathbb{R}^{6}$ and $\mathbf{J}_{\mathrm{te}} \in \mathbb{R}^{6 \times 6}$ are defined as:

$$
\begin{gathered}
\mathbf{M}_{\mathrm{te}}=\left[\begin{array}{cc}
m_{\mathrm{t}} \mathbf{E}_{3} & \mathbf{0} \\
\mathbf{0} & \mathbf{I}_{\mathrm{te}}
\end{array}\right] \quad \mathbf{c}_{\mathrm{te}}=\left[\begin{array}{l}
{ }^{\mathrm{e}} \mathbf{w}_{\mathrm{t}} \times m_{\mathrm{t}}{ }^{\mathrm{e}} \mathbf{v}_{\mathrm{t}} \\
{ }^{\mathrm{e}} \mathbf{w}_{\mathrm{t}} \times \mathbf{I}_{\mathrm{te}}{ }^{\mathrm{e}} \mathbf{w}_{\mathrm{t}}
\end{array}\right] \\
\mathbf{J}_{\mathrm{te}}=\left[\begin{array}{cc}
\mathbf{E}_{3} & -{ }^{\mathrm{e}} \mathbf{r}_{\mathrm{te}}^{\times} \\
\mathbf{0} & \mathbf{E}_{3}
\end{array}\right]
\end{gathered}
$$

and $m_{\mathrm{t}}$ and $\mathbf{I}_{\mathrm{te}} \in \mathbb{R}^{3 \times 3}$ are the mass and inertia tensor of the target about $\Sigma_{\mathrm{t}}$, respectively. ${ }^{\mathrm{e}} \mathbf{r}_{\mathrm{te}} \in \mathbb{R}^{3}$ is the radial vector from the target's center of mass to the grasping point, and ${ }^{\mathrm{e}} \mathbf{f}_{\mathrm{e}}$ is the end-effector's force/torque expressed in $\Sigma_{\mathrm{e}} \cdot{ }^{\mathrm{e}} \mathbf{f}_{\mathrm{e}}$ and $\mathbf{f}_{\mathrm{e}}$ have the following relationship:

$$
\begin{aligned}
{ }^{\mathrm{e}} \mathbf{f}_{\mathrm{e}} & =\overline{\mathbf{R}} \mathbf{f}_{\mathrm{e}} \\
& =\left[\begin{array}{cc}
{ }^{\mathrm{e}} \mathbf{R} & \mathbf{0} \\
\mathbf{0} & { }^{\mathrm{e}} \mathbf{R}
\end{array}\right] \mathbf{f}_{\mathrm{e}} .
\end{aligned}
$$

The velocity of the end-effector and target are subjected to the following kinematic constraints as a result of grasping (assumption 2):

$$
\begin{aligned}
& { }^{\mathrm{e}} \boldsymbol{v}_{\mathrm{e}}=\mathbf{J}_{\mathrm{te}}{ }^{\mathrm{e}} \boldsymbol{v}_{\mathrm{t}} \\
& \mathrm{e}^{\dot{\boldsymbol{v}}_{\mathrm{e}}}=\mathbf{J}_{\mathrm{te}}{ }^{\mathrm{t}} \dot{\boldsymbol{v}}_{\mathrm{t}}
\end{aligned}
$$

Utilizing (3.36) and 3.37) in (3.32) results in the following equation of motion for the target:

$$
\Lambda_{\mathrm{te}}{ }^{\mathrm{e}} \dot{\boldsymbol{v}}_{\mathrm{e}}+\mathbf{J}_{\mathrm{te}}^{-\mathrm{T}} \mathbf{c}_{\mathrm{te}}={ }^{\mathrm{e}} \mathbf{f}_{\mathrm{e}}
$$


where $\Lambda_{\mathrm{te}}=\mathbf{J}_{\mathrm{te}}^{-\mathrm{T}} \mathbf{M}_{\mathrm{te}} \mathbf{J}_{\mathrm{te}}^{-1} \in \mathbb{R}^{6 \times 6}$ represents the target's inertia projected onto the end-effector. $\Lambda_{\mathrm{te}}>0$ since $\mathbf{M}_{\mathrm{te}}>0$ and $\mathbf{J}_{\mathrm{te}}^{-1}$ is always full rank ( $\mathbf{J}_{\mathrm{te}}$ defined in 3.34 ) is always invertible).

\subsubsection{Modified Detumbling Control}

Recall that the ultimate goal of detumbling a target is to bring its linear and angular velocities to rest and is accomplished by applying a detumbling force/torque to the target at the grasping location. Achieving this goal is further complicated as the detumbling force/torque must be constrained by force/torque limits at the grasping location. As pointed out earlier, in the literature, this is accomplished by defining a desired detumbling force/torque as a function of the target's inertial parameters and force/torque limits [6,7, 9, 47, 65]. However, it is not practical to obtain accurate values of the target's inertial parameters for use in the computation of the desired detumbling force/torque. Furthermore, the detumbling force/torque presented by [6,7,, 9$]$ and [47] will result in a discontinuous desired detumbling force/torque. This is undesirable as the discontinuity may result in the excitation of unmodeled dynamics in both the servicer and target.

The detumbling force/torque presented in this section is designed to bring the target's linear and angular velocities to zero, and it does not require the target's inertial parameters in its computation and is continuous. This is accomplished by defining the desired force/torque in the opposite direction of the end-effector's linear/angular velocity vectors and scaled by force/torque limits. The desired force/torque is denoted as ${ }^{\mathrm{e}} \mathbf{f}_{\mathrm{eD}}=\left[{ }^{\mathrm{e}} \mathbf{F}_{\mathrm{eD}}^{\mathrm{T}},{ }_{\mathrm{eDD}}^{\mathrm{e}}\right]^{\mathrm{T}} \in \mathbb{R}^{6}$, where ${ }^{\mathrm{e}} \mathbf{F}_{\mathrm{eD}} \in \mathbb{R}^{3}$ denotes the end-effector's desired force and ${ }^{\mathrm{e}} \tau_{\mathrm{eD}} \in \mathbb{R}^{3}$ denotes the end-effector's desired toque. ${ }^{\mathrm{e}} \mathbf{F}_{\mathrm{eD}}$ and ${ }^{\mathrm{e}} \tau_{\mathrm{eD}}$ are defined as follows:

$$
\begin{aligned}
& { }^{\mathrm{e}} \mathbf{F}_{\mathrm{eD}}=\frac{{ }^{\mathrm{e}} \mathbf{v}_{\mathrm{e}}}{\left\|{ }^{\mathrm{e}} \mathbf{v}_{\mathrm{e}}\right\|+\varepsilon_{\mathrm{s}}}\left(-\mathrm{F}_{\max }\right) \\
& { }^{\mathrm{e}} \tau_{\mathrm{eD}}=\frac{{ }^{\mathrm{e}} \mathbf{w}_{\mathrm{e}}}{\left\|{ }^{\mathrm{e}} \boldsymbol{v}_{\mathrm{e}}\right\|+\varepsilon_{\mathrm{s}}}\left(-\tau_{\max }\right)
\end{aligned}
$$

where $F_{\max }$ and $\tau_{\max }$ are the force and torque limits, respectively, at the end-effector. $\varepsilon_{\mathrm{s}}$ is a positive constant used to attenuate the magnitude of the desired force and torque, respectively, as the end-effector's linear and angular velocities converge to zero. This was done to avoid chattering of the desired force/torque since kinematic coupling between the end-effector's linear and angular velocities, and that of the target's centre of mass will result in the following as 
the end-effector's linear and angular velocities converge to zero and if $\varepsilon_{\mathrm{s}}=0$ : end-effector's linear and angular velocities will not remain at zero unless both are zero.

The auxiliary input, $\mathbf{u}_{\mathrm{f}}$, is defined as follows:

$$
\begin{aligned}
\mathbf{u}_{\mathrm{f}} & =\overline{\mathbf{R}}^{\mathrm{T}} \mathbf{u}_{\mathrm{f}} \\
& =\overline{\mathbf{R}}^{\mathrm{T}}\left(\mathbf{K}_{\mathrm{fP}}\left({ }^{\mathrm{e}} \mathbf{f}_{\mathrm{eD}}-{ }^{\mathrm{e}} \mathbf{f}_{\mathrm{e}}\right)-\mathbf{K}_{\mathrm{fP}} \Psi\right)
\end{aligned}
$$

where $\mathbf{K}_{\mathrm{fP}} \in \mathbb{R}^{6 \times 6}>0$ is block diagonal proportional force error gain and $\Psi \in \mathbb{R}^{6}$ is a robust compensator that will account for the target's unknown inertial parameters (defined in the following subsection).

\subsubsection{Controller Evaluation}

Substituting (3.1), 3.3b) and (3.41) into the servicer's equation of motion, 2.36), and noting that $\dot{\boldsymbol{v}}_{\mathrm{e}}=\overline{\mathbf{R}}^{\mathrm{T}}\left({ }^{\mathrm{e}} \dot{\boldsymbol{v}}_{\mathrm{e}}+\mathbf{V}_{\mathrm{c}}\right)$, results in the following closed-loop equations:

$$
\begin{gathered}
{ }^{\mathrm{e}} \dot{\boldsymbol{v}}_{\mathrm{e}}=\mathbf{K}_{\mathrm{fP}}\left({ }^{\mathrm{e}} \mathbf{f}_{\mathrm{eD}}-{ }^{\mathrm{e}} \mathbf{f}_{\mathrm{e}}\right)-\mathbf{K}_{\mathrm{fP}} \Psi-\mathbf{V}_{\mathrm{c}} \\
\dot{\mathbf{w}}_{\mathrm{b}}=-\mathbf{K}_{\mathrm{AD}} \mathbf{w}_{\mathrm{b}}-\mathrm{K}_{\mathrm{AP}} \delta \mathbf{q}_{\mathrm{v}}
\end{gathered}
$$

where

$$
\mathbf{V}_{\mathrm{c}}=\left[\begin{array}{c}
\mathrm{e}_{\mathbf{w}_{\mathrm{e}}} \times \mathrm{e} \\
\mathbf{0}
\end{array}\right]
$$

Stability of system (3.43) is proven based on the Lyapunov argument presented by [73] (stability with the use of the base controller (3.3a) is trivial). Stability of (3.42) can be ascertained by proving that the target's kinetic energy decrease during the detumbling procedure. The target's kinetic energy is defined as:

$$
\mathrm{V}_{1}=\frac{1}{2} \mathrm{e}_{\mathrm{e}}^{\mathrm{T}} \Lambda_{\mathrm{te}} \mathrm{v}_{\mathrm{e}}
$$


Taking the time derivative of (3.45) and using (3.38), we have:

$$
\begin{aligned}
\dot{\mathrm{V}}_{1} & ={ }^{\mathrm{e}} \boldsymbol{v}_{\mathrm{e}}^{\mathrm{T}} \Lambda_{\mathrm{te}}{ }^{\mathrm{e}} \dot{\boldsymbol{v}}_{\mathrm{e}} \\
& ={ }^{\mathrm{e}} \boldsymbol{v}_{\mathrm{e}}^{\mathrm{T}}\left({ }^{\mathrm{e}} \mathbf{f}_{\mathrm{e}}-\mathbf{J}_{\mathrm{te}}^{-\mathrm{T}} \mathbf{c}_{\mathrm{te}}\right) \\
& ={ }^{\mathrm{e}} \boldsymbol{v}_{\mathrm{e}}^{\mathrm{Te}} \mathbf{f}_{\mathrm{e}}
\end{aligned}
$$

where ${ }^{\mathrm{e}} \boldsymbol{v}_{\mathrm{e}}^{\mathrm{T}} \mathbf{J}_{\mathrm{te}}^{-\mathrm{T}} \mathbf{c}_{\mathrm{te}}={ }^{\mathrm{e}} \boldsymbol{v}_{\mathrm{t}}^{\mathrm{T}} \mathbf{J}_{\text {te }}^{\mathrm{T}} \mathbf{J}_{\mathrm{te}}^{-\mathrm{T}} \mathbf{c}_{\mathrm{te}}=0$ with the use of 3.36 .

Evaluating $\dot{V}_{1}$ in 3.46 requires an expression of the end-effector's force/torque, ${ }^{e} \mathbf{f}_{\mathrm{e}}$, that encompasses the desired force/torque and the force/torque error. This is accomplished by rearranging the target's equation of motion in (3.38), resulting in:

$$
\begin{gathered}
\mathrm{e}_{\dot{\boldsymbol{v}}_{\mathrm{e}}}=\Lambda_{\mathrm{te}}^{-1 \mathrm{e}} \mathbf{f}_{\mathrm{e}}-\Lambda_{\mathrm{te}}^{-1} \mathbf{J}_{\mathrm{te}}^{-\mathrm{T}} \mathbf{c}_{\mathrm{te}} \\
=\Lambda_{\mathrm{te}}^{-1 \mathrm{e}} \mathbf{f}_{\mathrm{e}}-f\left(\mathbf{M}_{\mathrm{te}},{ }^{\mathrm{e}} \mathbf{r}_{\mathrm{te}},{ }^{\mathrm{e}} \boldsymbol{v}_{\mathrm{e}}\right)-\mathbf{V}_{\mathrm{c}} \\
f\left(\mathbf{M}_{\mathrm{te}},{ }^{\mathrm{e}} \mathbf{r}_{\mathrm{te}},{ }^{\mathrm{e}} \boldsymbol{v}_{\mathrm{e}}\right)=\left[\begin{array}{c}
{ }^{\mathrm{e}} \mathbf{w}_{\mathrm{e}} \times{ }^{\times} \mathbf{r}_{\mathrm{te}}^{\times \mathrm{e}} \mathbf{w}_{\mathrm{e}} \\
\mathbf{0}
\end{array}\right]+\left[\begin{array}{c}
-{ }^{\mathrm{e}} \mathbf{r}_{\mathrm{te}}^{\times} \mathbf{I}_{\mathrm{te}}^{-1 \mathrm{e}} \mathbf{w}_{\mathrm{e}}^{\times} \mathbf{I}_{\mathrm{te}}{ }^{\mathrm{e}} \mathbf{w}_{\mathrm{e}} \\
\mathbf{I}_{\mathrm{te}}^{-1 \mathrm{e}} \mathbf{w}_{\mathrm{e}}^{\times} \mathbf{I}_{\mathrm{te}}{ }^{\mathrm{e}} \mathbf{w}_{\mathrm{e}}
\end{array}\right]
\end{gathered}
$$

where ${ }^{\mathrm{e}} \mathbf{w}_{\mathrm{e}}={ }^{\mathrm{e}} \mathbf{w}_{\mathrm{t}}$ as per 3.36). Substituting 3.47) into the closed-loop equation, 3.42, results in the following expression for the end-effector's force/torque:

$$
\begin{gathered}
{ }^{\mathrm{e}} \mathbf{f}_{\mathrm{e}}={ }^{\mathrm{e}} \mathbf{f}_{\mathrm{eD}}+\mathbf{K}_{\mathrm{fP}}^{-1} \mathbf{G}-\Psi \\
\mathbf{G}=f\left(\mathbf{M}_{\mathrm{te}},{ }^{\mathrm{e}} \mathbf{r}_{\mathrm{te}},{ }^{\mathrm{e}} \boldsymbol{v}_{\mathrm{e}}\right)-\Lambda_{\mathrm{te}}^{-1 \mathrm{e}} \mathbf{f}_{\mathrm{e}}
\end{gathered}
$$

From 3.49a), the term $\mathbf{G}$ prevents the end-effector's force/torque, ${ }^{\mathrm{e}} \mathbf{f}_{\mathrm{e}}$, from accurately tracking the desired force/torque, ${ }^{\mathrm{e}} \mathbf{f}_{\mathrm{eD}}$. The additional term $\mathbf{G}$ is a function of the target's inertial parameters and may cause the target's kinetic energy to increase during the detumbling procedure. An increase in the target's kinetic energy is undesirable in the detumbling procedure as it may result in a prolonged detumbling process. In addition, the evolution of the target's motion subjected to an increase in kinetic energy is difficult to predict and may result in the target rotating out of the workspace of the servicer's manipulator. Thus, it is desirable for the target's kinetic energy to monotonically converge to zero. To achieve this, the robust compensator, $\Psi$, is designed to robustly compensate for $\mathbf{G}$ so that the target's kinetic energy 
monotonically converges to zero. Similar to the work by [28], the robust compensator, $\Psi$, in 3.49a is defined as:

$$
\Psi_{i}=\mathrm{D}_{i} \tanh \left(\frac{6 \mathrm{D}_{i} \mathrm{k}_{\mathrm{u}}\left\{{ }_{\boldsymbol{v}_{\mathrm{e}}}\right\}_{i}}{\varepsilon_{\mathrm{r}}}\right) \quad \text { for } i=1,2,3, \ldots, 6
$$

where $\mathrm{k}_{\mathrm{u}}=0.2785$ [52], $\varepsilon_{\mathrm{r}}$ is a small positive scalar, and $\mathrm{D}_{i}$ is a bounding function of the end-effector's angular velocity, end-effector's force/torque and bounds on the target's inertial parameters, and satisfies the following inequality:

$$
\left|\left\{\mathbf{K}_{\mathrm{fP}}^{-1} \mathbf{G}\right\}_{i}\right| \leq \mathrm{D}_{i}
$$

Defining the robust compensator, 3.50a, with the use of the hyperbolic tangent function was done to prevent chattering. That is, if the hyperbolic tangent function was not utilized, an alternative robust compensator would have to be defined using the sign function of the endeffector velocity and would result in chattering of the robust compensator: $\Psi_{i}=\mathrm{D}_{i} \operatorname{sgn}\left(\left\{{ }^{\mathrm{e}} \mathrm{v}_{\mathrm{e}}\right\}_{i}\right)$, where $\operatorname{sgn}(x)=1$ if $x>0, \operatorname{sgn}(x)=-1$ if $x<0$, or $\operatorname{sgn}(x)=0$ if $x=0$. Chattering would result as the individual components of the end-effector's velocity will not converge to zero all at the same time. Some components would equal to zero while others would not. This arises because there is coupling between the servcier's end-effector's velocity and target's center of mass velocity, 2.40), the components at zero will not stay at zero unless all components of the end-effector's velocity are all zero. Using the sign function in the robust compensator, $\Psi_{i}$, would result in its values changing from 0 to $\mathrm{D}_{i}$ when the $\left|\left\{{ }^{\mathrm{e}} \boldsymbol{v}_{\mathrm{e}}\right\}_{i}\right|$ changes from 0 to a nonzero value. This is undesirable as this sudden change in control input could excite unmodelled dynamics.

To determine the bounding function, $\mathrm{D}_{i}$, note that $\mathbf{G} \in \mathbb{R}^{6}$ in $3.49 \mathrm{~b}$ can be segmented into linear and angular components as follow: $\mathbf{G}=\left[\mathbf{G}_{\mathrm{L}}^{\mathrm{T}}, \mathbf{G}_{\mathrm{A}}^{\mathrm{T}}\right]^{\mathrm{T}}$ where $\mathbf{G}_{\mathrm{L}} \in \mathbb{R}^{3}$ and $\mathbf{G}_{\mathrm{A}} \in \mathbb{R}^{3}$ are the linear and angular components of $\mathbf{G}$, respectively. Using (3.48) and the definition of $\Lambda_{\mathrm{t}}$ from (3.38) in 3.49b), allows $\mathbf{G}$ to be expanded as:

$$
\begin{aligned}
\mathbf{G}_{\mathrm{L}}= & { }^{\mathrm{e}} \mathbf{w}_{\mathrm{e}}^{\times \mathrm{e}} \mathbf{r}_{\mathrm{te}}^{\times \mathrm{e}} \mathbf{w}_{\mathrm{e}}-{ }^{\mathrm{e}} \mathbf{r}_{\mathrm{te}}^{\times} \mathbf{I}_{\mathrm{te}}^{-1 \mathrm{e}} \mathbf{w}_{\mathrm{e}}^{\times} \mathbf{I}_{\mathrm{ee}}{ }^{\mathrm{e}} \mathbf{w}_{\mathrm{e}} \\
& -\left(m_{\mathrm{t}}^{-1} \mathbf{E}_{3}-{ }^{\mathrm{e}} \mathbf{r}_{\mathrm{te}}^{\times} \mathbf{I}_{\mathrm{te}}^{-1 \mathrm{e}} \mathbf{r}_{\mathrm{te}}^{\times}\right)^{\mathrm{e}} \mathbf{F}_{\mathrm{e}}+{ }^{\mathrm{e}} \mathbf{r}_{\mathrm{te}}^{\times} \mathbf{I}_{\mathrm{te}}^{-1 \mathrm{e}} \tau_{\mathrm{e}} \\
\mathbf{G}_{\mathrm{A}}= & \mathbf{I}_{\mathrm{te}}^{-1 \mathrm{e}} \mathbf{w}_{\mathrm{e}}^{\times} \mathbf{I}_{\mathrm{te}}{ }^{\mathrm{e}} \mathbf{w}_{\mathrm{e}}-\mathbf{I}_{\mathrm{te}}^{-1 \mathrm{e}} \mathbf{r}_{\mathrm{te}}^{\times \mathrm{e}} \mathbf{F}_{\mathrm{e}}-\mathbf{I}_{\mathrm{te}}^{-1 \mathrm{e}} \tau_{\mathrm{e}}
\end{aligned}
$$


$\mathrm{D}_{i}$ can be determined by bounding $\mathbf{G}_{\mathrm{L}}$ and $\mathbf{G}_{\mathrm{A}}$. This is established by noting the following:

$$
\left\|{ }^{\mathrm{e}} \mathbf{r}_{\mathrm{te}}\right\| \leq r_{\mathrm{te}},
$$

and make use of Lemma 1 in [77]. As a result, the components of $\mathbf{G}_{\mathrm{L}}$ and $\mathbf{G}_{\mathrm{A}}$ are bounded as follows:

$$
\begin{aligned}
&{ }^{\mathrm{e}} \mathbf{w}_{\mathrm{e}}^{\times \mathrm{e}} \mathbf{r}_{\mathrm{te}}^{\times \mathrm{e}} \mathbf{w}_{\mathrm{e}} \leq\left\|{ }^{\mathrm{e}} \mathbf{w}_{\mathrm{e}}\right\|^{2} r_{\mathrm{te}} \\
&-{ }^{\mathrm{e}} \mathbf{r}_{\mathrm{te}}^{\times} \mathbf{I}_{\mathrm{te}}^{-1 \mathrm{e}} \mathbf{w}_{\mathrm{e}}^{\times} \mathbf{I}_{\mathrm{te}}{ }^{\mathrm{e}} \mathbf{w}_{\mathrm{e}}=\left(\mathbf{I}_{\mathrm{te}}^{-1 \mathrm{e}} \mathbf{w}_{\mathrm{e}}^{\times} \mathbf{I}_{\mathrm{te}}{ }^{\mathrm{e}} \mathbf{w}_{\mathrm{e}}\right)^{\times \mathrm{e}} \mathbf{r}_{\mathrm{te}} \\
& \leq\left\|\mathbf{I}_{\mathrm{te}}^{-1}\right\|\left[\lambda_{\mathrm{max}}^{2}\left(\mathbf{I}_{\mathrm{te}}\right)-\lambda_{\min }^{2}\left(\mathbf{I}_{\mathrm{te}}\right)\right]^{1 / 2}\left\|^{\mathrm{e}} \mathbf{w}_{\mathrm{e}}\right\|^{2} r_{\mathrm{te}} \\
& \leq\left[\sigma^{2}-1\right]^{1 / 2}\left\|{ }^{\mathrm{e}} \mathbf{w}_{\mathrm{e}}\right\|^{2} r_{\mathrm{te}}
\end{aligned}
$$

where $\sigma=\lambda_{\max }\left(\mathbf{I}_{\mathrm{te}}\right) / \lambda_{\min }\left(\mathbf{I}_{\mathrm{te}}\right)$.

$$
\begin{aligned}
&-\left(m_{\mathrm{t}}^{-1} \mathbf{E}_{3}-{ }^{\mathrm{e}} \mathbf{r}_{\mathrm{te}}^{\times} \mathbf{I}_{\mathrm{te}}^{-1 \mathrm{e}} \mathbf{r}_{\mathrm{te}}^{\times}\right) \mathbf{F}_{\mathrm{e}} \leq m_{\mathrm{t}}^{-1}\left\|\mathbf{F}_{\mathrm{e}}\right\|+r_{\mathrm{te}}\left\|{ }^{\mathrm{e}} \mathbf{r}_{\mathrm{te}}^{\times} \mathbf{F}_{\mathrm{e}}\right\| \lambda_{\min }^{-1}\left(\mathbf{I}_{\mathrm{te}}\right) \leq\left(m_{\mathrm{t}}^{-1}+r_{\mathrm{te}}{ }^{2} \lambda_{\min }^{-1}\left(\mathbf{I}_{\mathrm{te}}\right)\right)\left\|\mathbf{F}_{\mathrm{e}}\right\| \\
&{ }^{\mathrm{e}} \mathbf{r}_{\mathrm{te}}^{\times} \mathbf{I}_{\mathrm{te}}^{-1} \tau_{\mathrm{e}} \leq r_{\mathrm{te}} \lambda_{\min }^{-1}\left(\mathbf{I}_{\mathrm{t}}\right)\left\|\tau_{\mathrm{e}}\right\| \\
& \mathbf{I}_{\mathrm{te}}^{-1 \mathrm{e}} \mathbf{w}_{\mathrm{e}}^{\times} \mathbf{I}_{\mathrm{te}}{ }^{\mathrm{e}} \mathbf{w}_{\mathrm{e}} \leq\left[\sigma^{2}-1\right]^{1 / 2}\left\|^{\mathrm{e}} \mathbf{w}_{\mathrm{e}}\right\|^{2} \\
&-\mathbf{I}_{\mathrm{te}}^{-1 \mathrm{e}} \mathbf{r}_{\mathrm{te}}^{\times} \mathbf{F}_{\mathrm{e}}-\mathbf{I}_{\mathrm{te}}^{-1} \tau_{\mathrm{e}} \leq \lambda_{\min }^{-1}\left(\mathbf{I}_{\mathrm{te}}\right)\left[r_{\mathrm{te}}\left\|\mathbf{F}_{\mathrm{e}}\right\|+\left\|\tau_{\mathrm{e}}\right\|\right]
\end{aligned}
$$

Using the defined bounds in 3.53 3.58) to upper bound $\mathbf{G}_{\mathrm{L}}$ and $\mathbf{G}_{\mathrm{A}}$ in 3.51 , the following bounding function, $\mathrm{D}_{i}$, can be obtained:

$$
\begin{gathered}
\mathrm{D}_{i=1,2,3}=\left\|\mathbf{K}_{\mathrm{fP} i}^{-1}\right\|\left[a_{1}\left\|{ }^{\mathrm{e}} \mathbf{w}_{\mathrm{e}}\right\|^{2}+a_{2}\left\|{ }^{\mathrm{e}} \mathbf{F}_{\mathrm{e}}\right\|+a_{3}\left\|^{\mathrm{e}} \tau_{\mathrm{e}}\right\|\right] \\
\mathrm{D}_{i=4,5,6}=\left\|\mathbf{K}_{\mathrm{fP} i}^{-1}\right\|\left[a_{4}\left\|^{\mathrm{e}} \mathbf{w}_{\mathrm{e}}\right\|^{2}+a_{5}\left\|^{\mathrm{e}} \mathbf{F}_{\mathrm{e}}\right\|+a_{6}\left\|^{\mathrm{e}} \tau_{\mathrm{e}}\right\|\right]
\end{gathered}
$$


where

$$
\begin{gathered}
a_{1}=r_{\mathrm{te}}\left(\left(\sigma^{2}-1\right)^{1 / 2}+1\right) \\
a_{2}=\left(m_{\mathrm{t}}^{-1}+r_{\mathrm{te}}^{2} \lambda_{\min }^{-1}\left(\mathbf{I}_{\mathrm{te}}\right)\right) \\
a_{3}=\left(r_{\mathrm{te}} \lambda_{\min }^{-1}\left(\mathbf{I}_{\mathrm{te}}\right)\right) \\
a_{4}=\left(\sigma^{2}-1\right)^{1 / 2} \\
a_{5}=a_{3} \\
a_{6}=\lambda_{\operatorname{minL}}^{-1}\left(\mathbf{I}_{\mathrm{te}}\right)
\end{gathered}
$$

Now, $\dot{\mathrm{V}}_{1}$ can be evaluated by substituting 3.49a into 3.46, resulting in:

$$
\dot{\mathrm{V}}_{1}={ }^{\mathrm{e}} \boldsymbol{v}_{\mathrm{e}}^{\mathrm{Te}} \mathbf{f}_{\mathrm{eD}}+{ }^{\mathrm{e}} \boldsymbol{v}_{\mathrm{e}}^{\mathrm{T}}\left(\mathbf{K}_{\mathrm{fP}}^{-1} \mathbf{G}-\Psi\right)
$$

An upper bound on first term of 3.61 is obtained as follows:

$$
\begin{aligned}
{ }^{\mathrm{e}} \boldsymbol{v}_{\mathrm{e}}^{\mathrm{Te}} \mathbf{f}_{\mathrm{eD}} & =-\left(\left\|{ }^{\mathrm{e}} \mathbf{v}_{\mathrm{e}}\right\|^{2} \mathrm{~F}_{\max }+\left\|{ }^{\mathrm{e}} \mathbf{w}_{\mathrm{e}}\right\|^{2} \tau_{\max }\right) \frac{1}{\left\|\boldsymbol{v}_{\mathrm{e}}\right\|+\varepsilon_{\mathrm{s}}} \\
& \leq-\mathrm{K} \frac{\left\|{ }^{\mathrm{e}} \boldsymbol{v}_{\mathrm{e}}\right\|^{2}}{\left\|{ }^{\mathrm{e}} \boldsymbol{v}_{\mathrm{e}}\right\|+\varepsilon_{\mathrm{s}}}
\end{aligned}
$$

where $\left\|{ }^{\mathrm{e}} \boldsymbol{v}_{\mathrm{e}}\right\|^{2}=\left\|{ }^{\mathrm{e}} \mathbf{v}_{\mathrm{e}}\right\|^{2}+\left\|^{\mathrm{e}} \mathbf{w}_{\mathrm{e}}\right\|^{2}$ and $\mathrm{K}=\min \left\{\mathrm{F}_{\max }, \tau_{\max }\right\}$ were utilized.

The hyperbolic tangent function has the following property [52]:

$$
0 \leq|x|-x \tanh \left(\frac{x}{\varepsilon_{\mathrm{u}}}\right) \leq \mathrm{k}_{\mathrm{u}} \varepsilon_{\mathrm{u}}
$$

for any $\varepsilon_{\mathrm{u}}>0$ and any $x \in \mathbb{R}$. The second term of (3.61) is upper bounded with the use of 
(3.63) and (3.50) as follows:

$$
\begin{aligned}
{ }^{\mathrm{e}} \boldsymbol{v}_{\mathrm{e}}^{\mathrm{T}}\left(\mathbf{K}_{\mathrm{fP}}^{-1} \mathbf{G}-\Psi\right) & \leq \sum_{i}^{6}\left[\left|\left\{{ }^{\mathrm{e}} \boldsymbol{v}_{\mathrm{e}}\right\}_{i}\right| \mathrm{D}_{i}-{ }^{\mathrm{e}} \boldsymbol{v}_{\mathrm{e} i} \mathrm{D}_{i} \tanh \left(\frac{\left.6 \mathrm{D}_{i} \mathrm{k}_{\mathrm{u}}{ }^{\mathrm{e}}{ }_{\boldsymbol{v}_{\mathrm{e}}}\right\}_{i}}{\varepsilon_{\mathrm{r}}}\right)\right] \\
& \leq \sum_{i}^{6}\left(\frac{\varepsilon_{\mathrm{r}}}{6}\right) \\
& =\varepsilon_{\mathrm{r}}
\end{aligned}
$$

Utilizing 3.62 and 3.64 in 3.61, we obtain the following upper bound for $\dot{\mathrm{V}}_{1}$ :

$$
\dot{\mathrm{V}}_{1} \leq-\mathrm{K} \frac{\left\|{ }^{\mathrm{e}} \boldsymbol{v}_{\mathrm{e}}\right\|^{2}}{\left\|\boldsymbol{v}_{\mathrm{e}}\right\|+\varepsilon_{\mathrm{s}}}+\varepsilon_{\mathrm{r}} .
$$

From (3.65), the magnitude of the end-effector's velocity will converge to a nieghborbood of zero. The size of this neighborhood is determined by making use of the following inequality:

$$
\frac{\lambda_{\min \mathrm{A}}}{2}\left\|{ }^{\mathrm{e}} \boldsymbol{v}_{\mathrm{e}}\right\|^{2} \leq \mathrm{V}_{1} \leq \frac{\lambda_{\operatorname{maxA}}}{2}\left\|{ }^{\mathrm{e}} \boldsymbol{v}_{\mathrm{e}}\right\|^{2}
$$

where $\lambda_{\min A}$ and $\lambda_{\operatorname{maxA}}$ are the minimum amd maximum eigenvalue of $\Lambda_{\mathrm{te}}$.

Utilizing the inequality in $(3.66),(3.65)$ can be expressed as:

$$
\dot{\mathrm{V}}_{1} \leq-\frac{2 \mathrm{KV}_{1} / \lambda_{\operatorname{maxA}}}{\sqrt{2 \mathrm{~V}_{1} / \lambda_{\min \mathrm{A}}}+\varepsilon_{\mathrm{s}}}+\varepsilon_{\mathrm{r}}
$$

From 3.67 , $\dot{\mathrm{V}}_{1}<0$ when:

$$
\mathrm{V}_{1}>\left(\frac{\varepsilon_{\mathrm{r}} \sqrt{2 / \lambda_{\min \mathrm{A}}}+\sqrt{2 \varepsilon_{\mathrm{r}}^{2} / \lambda_{\min \mathrm{A}}+8 \varepsilon_{\mathrm{r}} \varepsilon_{\mathrm{s}} \mathrm{K} / \lambda_{\operatorname{maxA}}}}{4 \mathrm{~K} / \lambda_{\max \mathrm{A}}}\right)^{2} .
$$

Utilizing (3.66) and (3.68), an ultimate bound on the end-effector's velocity is:

$$
\begin{gathered}
\limsup _{t \rightarrow \infty}\left\|^{\mathrm{e}} \boldsymbol{v}_{\mathrm{e}}\right\| \leq \mathrm{C} \sqrt{2 / \lambda_{\min \mathrm{A}}} \\
\mathrm{C}=\left(\frac{\varepsilon_{\mathrm{r}} \sqrt{2 / \lambda_{\min \mathrm{A}}}+\sqrt{2 \varepsilon_{\mathrm{r}}{ }^{2} / \lambda_{\min \mathrm{A}}+8 \varepsilon_{\mathrm{r}} \varepsilon_{\mathrm{s}} \mathrm{K} / \lambda_{\operatorname{maxA}}}}{4 \mathrm{~K} / \lambda_{\operatorname{maxA}}}\right) .
\end{gathered}
$$

The proposed detumbling controller ensures that the end-effector's velocity will converge to a neighbourhood of $\left\|{ }^{\mathrm{e}} \boldsymbol{v}_{\mathrm{e}}\right\|=0$ and is ultimately upper bounded by 3.69). Both $\varepsilon_{\mathrm{r}}$ and $\varepsilon_{\mathrm{s}}$ are 
selected to avoid chatter of the proposed detumbling controller. $\varepsilon_{\mathrm{s}}$ is utilized to avoid chattering of ${ }^{\mathrm{e}} \mathbf{f}_{\mathrm{eD}}$ and $\varepsilon_{\mathrm{r}}$ is utilized to avoid chattering of the robust compensator. As shown from 3.69), a reduction in $\varepsilon_{\mathrm{r}}$ will result in a reduced ultimate upper bound on the end-effector's velocity. However, this increases the likelihood of chattering when the end-effector's linear and angular velocities are near zero. Thus, the selection of $\varepsilon_{\mathrm{r}}$ must trade off between the magnitude of the upper bound on $\left\|{ }^{\mathrm{e}} \boldsymbol{v}_{\mathrm{e}}\right\|$ and chattering effect of the control.

\subsubsection{Real-Time Estimation of Bounds on the Target's Inertial Parame- ters}

The robust compensator, $\Psi$ in $3.50 \mathrm{a}$, requires bounds on the target's inertial parameters in order to reject the disturbance created by $\mathbf{G}_{\mathrm{L}}$ and $\mathbf{G}_{\mathrm{A}}$. The target's inertial parameters are unknown, but bounds on the target's inertial parameters can be estimated from pre-launch data. Traditionally, these estimates will be conservative and will result in larger than required robust gains to detumble the target. An intuitive way to avoid this and enhance robustness, is to introduce an additional control input that adaptively rejects the disturbance created by $\mathbf{G}_{\mathrm{L}}$ and $\mathbf{G}_{\mathrm{A}}$. This is achieved by estimating the bounds on the target's inertial parameter, $a_{i}$, in real-time and ensuring that the estimate is always less than or equal to the conservative estimate of $a_{i}$, denoted as $\bar{a}_{i}$.

With additional modelling information, the target's inertial parameters can be bounded as:

$$
\begin{gathered}
m_{\mathrm{tL}} \leq m_{\mathrm{t}} \leq m_{\mathrm{tU}} \\
\lambda_{\operatorname{maxL}} \leq \lambda_{\max }\left(\mathbf{I}_{\mathrm{te}}\right) \leq \lambda_{\operatorname{maxU}} \\
\lambda_{\operatorname{minL}} \leq \lambda_{\min }\left(\mathbf{I}_{\mathrm{te}}\right) \leq \lambda_{\operatorname{minU}} \\
r_{\mathrm{teL}} \leq\left\|\mathbf{r}_{\mathrm{te}}\right\| \leq r_{\mathrm{teU}}
\end{gathered}
$$

where $(\cdot)_{\mathrm{L}}$ and $(\cdot)_{\mathrm{U}}$ represent lower and upper bounds on $(\cdot)$, respectively. Utilize 3.70 , the coefficients, $a_{i}$, of the bounding function, $\mathrm{D}_{i}$, defined in 3.60) can be upper and lower bounded such that $\underline{a}_{i} \leq a_{i} \leq \bar{a}_{i}$ for $i=1,2,3, \ldots, 6$, where $\underline{a}_{i}$ and $\bar{a}_{i}$ represent the lower and upper bounds 
of $a_{i}$, respectively. As a result, the robust compensator is designed as:

$$
\Psi_{i}=2 \hat{\mathrm{D}}_{i} \tanh \left(\frac{12 \hat{\mathrm{D}}_{i} \mathrm{k}_{\mathrm{u}}\left\{\mathrm{e}_{\mathrm{e}}\right\}_{i}}{\mathcal{E}_{\mathrm{r}}}\right) \quad \text { for } i=1,2,3, \ldots, 6
$$

where $\hat{\mathrm{D}}_{i}$ is computed as in 3.59 with the use of $\hat{a}_{i}$ instead of $a_{i}$. The variable $\hat{a}_{i}$ is a dynamic estimate of $a_{i}$ for $i=1,2,3, \ldots, 6$, and are updated by [24]:

$$
\dot{\hat{a}}_{i}=c_{i}\left(1-\frac{\hat{a}_{i}}{\bar{a}_{i}}\right) \rho_{i}-\kappa_{i}\left(\hat{a}_{i}-\underline{a}_{i}\right) \quad \text { for } i=1,2,3, \ldots, 6
$$

where $c_{i}>0$ and $\kappa_{i}>0$ are constants and $\rho_{i}$ for $i=1,2,3, \ldots, 6$ are defined as follows:

$$
\begin{aligned}
& \rho_{i}=\Xi_{\star}\left\|\mathbf{K}_{\mathrm{fPi}}^{-1}\right\|\left\|{ }^{\mathrm{e}} \mathbf{w}_{\mathrm{e}}\right\|^{2} \quad \text { for } i=1,4 \\
& \rho_{i}=\Xi_{\star}\left\|\mathbf{K}_{\mathrm{fPi}}^{-1}\right\|\left\|\left.\right|^{\mathrm{e}} \mathbf{F}_{\mathrm{e}}\right\| \quad \text { for } i=2,5 \\
& \rho_{i}=\Xi_{\star}\left\|\mathbf{K}_{\mathrm{fPi}}^{-1}\right\|\|\|^{\mathrm{e}} \tau_{\mathrm{e}} \| \quad \text { for } i=3,6
\end{aligned}
$$

where $\star=U$ if $i=1,2,3$, and $\star=L$ if $i=4,5,6 . \Xi_{U}=\sum_{i=1}^{3}\left|\left\{{ }^{\mathrm{e}} \boldsymbol{v}_{\mathrm{e}}\right\}_{i}\right|$ and $\Xi_{L}=\sum_{i=4}^{6}\left|\left\{{ }_{\mathrm{e}} \boldsymbol{v}_{\mathrm{e}}\right\}_{i}\right|$. The above adaptive law (3.72) will ensure that $\underline{a}_{i} \leq \hat{a}_{i}(t) \leq \bar{a}_{i}$ if $\underline{a}_{i} \leq \hat{a}_{i}(0) \leq \bar{a}_{i}$ and thus prevents the estimate, $\hat{a}_{i}$, from drifting arbitrarily and exceeding the conservative estimate, $\bar{a}_{i}$ [24].

Stability of the resultant system 3.42 with the modified robust compensator, 3.71], and adaptive update law, (3.72), is studied considering the following Lyapunov function candidate:

$$
\mathrm{V}_{2}=\mathrm{V}_{1}+\sum_{i=1}^{6} \frac{1}{2 c_{i}} \tilde{a}_{i}^{2}
$$

where $\tilde{a}_{i}=a_{i}-\hat{a}_{i}$ and $\mathrm{V}_{1}$ was defined in (3.45).

Taking the time derivative of (3.74) and using (3.38) and (3.49a), we have:

$$
\dot{\mathrm{V}}_{2}={ }^{\mathrm{e}} \boldsymbol{v}_{\mathrm{e}}^{\mathrm{Te}} \mathbf{f}_{\mathrm{eD}}+{ }^{\mathrm{e}} \boldsymbol{v}_{\mathrm{e}}^{\mathrm{T}}\left(\mathbf{K}_{\mathrm{fP}}^{-1} \mathbf{G}-\Psi\right)-\sum_{i=1}^{6} \frac{1}{c_{i}} \tilde{a}_{i} \dot{\hat{a}}_{i}
$$

where $\Psi$ is defined as per 3.71.

The first term of (3.75) is upper bounded as in (3.62) and the second term of (3.75) is upper 
bounded with the use of 3.73 , 3.71, 3.63 and $3.50 \mathrm{~b})$ as follows:

$$
\begin{aligned}
& { }^{\mathrm{e}} \boldsymbol{v}_{\mathrm{e}}^{\mathrm{T}}\left(\mathbf{K}_{\mathrm{fP}}^{-1} \mathbf{G}-\Psi\right) \leq \sum_{i}^{6}\left[\left|\left\{{ }^{\mathrm{e}} \boldsymbol{v}_{\mathrm{e}}\right\}_{i}\right| \mathrm{D}_{i}-2^{\mathrm{e}} \boldsymbol{v}_{\mathrm{e} i} \hat{\mathrm{D}}_{i} \tanh \left(\frac{12 \hat{\mathrm{D}}_{i} \mathrm{k}_{\mathrm{u}}\left\{\boldsymbol{e}_{\mathrm{e}}\right\}_{i}}{\mathcal{\varepsilon}_{\mathrm{r}}}\right)\right] \\
& \leq \varepsilon_{\mathrm{r}}+\sum_{i=1}^{6}\left|\left\{{ }^{\mathrm{e}} \boldsymbol{v}_{\mathrm{e}}\right\}_{i}\right| \mathrm{D}_{i}-2 \sum_{i=1}^{6}\left|\left\{\mathrm{e}_{\mathrm{e}}\right\}_{i}\right| \hat{\mathrm{D}}_{i} \\
& =\varepsilon_{\mathrm{r}}+\sum_{i=1}^{6} a_{i} \rho_{i}-2 \sum_{i=1}^{6} \hat{a}_{i} \rho_{i}
\end{aligned}
$$

where $\sum_{i=1}^{6}\left|\left\{{ }^{\mathrm{e}} \boldsymbol{v}_{\mathrm{e}}\right\}_{i}\right| \mathrm{D}_{i}=\sum_{i=1}^{6} a_{i} \rho_{i}$ and $\sum_{i=1}^{6}\left|\left\{{ }^{\mathrm{e}} \boldsymbol{v}_{\mathrm{e}}\right\}_{i}\right| \hat{\mathrm{D}}_{i}=\sum_{i=1}^{6} \hat{a}_{i} \rho_{i}$ were utilized.

Since the adaptive law ensures $\underline{a}_{i} \leq \hat{a}_{i}(t) \leq \bar{a}_{i}$, it follows that $a_{i} / \bar{a}_{i} \leq 1, \hat{a}_{i}^{2} \geq \underline{a}_{i}^{2}$ and $\tilde{a}_{i}\left(\hat{a}_{i}-\right.$ $\left.\underline{a}_{i}\right) \leq\left(\bar{a}_{i}-\underline{a}_{i}\right)^{2}$. Using these properties along with 3.72 , the third term of 3.75 is upper bounded as follows:

$$
\begin{aligned}
-\sum_{i=1}^{6} \frac{1}{c_{i}} \tilde{a}_{i} \dot{\hat{a}}_{i} & =\sum_{i=1}^{6}\left(-\tilde{a}_{i} \rho_{i}+\frac{\tilde{a}_{i} \hat{a}_{i}}{\bar{a}_{i}} \rho_{i}+\frac{\kappa_{i}}{c_{i}} \tilde{a}_{i}\left(\hat{a}_{i}-\underline{a}_{i}\right)\right) \\
& \leq \sum_{i=1}^{6}\left(-a_{i} \rho_{i}+2 \hat{a}_{i} \rho_{i}-\frac{a_{i}^{2}}{\bar{a}_{i}} \rho_{i}+\frac{\kappa_{i}}{c_{i}}\left(\bar{a}_{i}-\underline{a}_{i}\right)^{2}\right) .
\end{aligned}
$$

Utilizing (3.62), 3.76) and 3.777 in 3.75) results in the following:

$$
\dot{\mathrm{V}}_{2} \leq-\mathrm{K} \frac{\left\|{ }^{\mathrm{e}} \boldsymbol{v}_{\mathrm{e}}\right\|^{2}}{\left\|\boldsymbol{v}_{\mathrm{e}}\right\|+\varepsilon_{\mathrm{s}}}+\eta_{\mathrm{o}}
$$

where $\eta_{o}=\varepsilon_{\mathrm{r}}+\sum_{i=1}^{6}\left(\frac{\kappa_{i}}{c_{i}}\left(\bar{a}_{i}-\underline{a}_{i}\right)^{2}\right)$. From 3.78 the magnitude of end-effector's velocity will converge to a neighborhood about zero. The size of this neighborhood is determined by making using of the following inequality:

$$
\frac{\lambda_{\operatorname{minA}}}{2}\left\|{ }^{\mathrm{e}} \boldsymbol{v}_{\mathrm{e}}\right\|^{2} \leq \mathrm{V}_{2} \leq \frac{\lambda_{\operatorname{maxA}}}{2}\left\|{ }^{\mathrm{e}} \boldsymbol{v}_{\mathrm{e}}\right\|^{2}+\sum_{i=1}^{6}\left|\tilde{a}_{i}\right|^{2} \leq \frac{\lambda_{\max }}{2}\left\|{ }^{\mathrm{e}} \boldsymbol{v}_{\mathrm{e}}\right\|^{2}+\sum_{i=1}^{6}\left(\bar{a}_{i}-\underline{a}_{i}\right)^{2}
$$

where $\left|\tilde{a}_{i}\right| \leq\left(\bar{a}_{i}-\underline{a}_{i}\right)$.

Similarly to Section 3.4.3, the ultimate bound on the end-effector velocity is obtained by using (3.79) in 3.78), resulting in the following:

$$
\begin{gathered}
\limsup _{t \rightarrow \infty}\left\|{ }^{\mathrm{e}} \boldsymbol{v}_{\mathrm{e}}\right\| \leq \mathrm{C} \sqrt{2 / \lambda_{\min \mathrm{A}}} \\
\mathrm{C}=\left(\frac{\eta_{\mathrm{o}} \sqrt{2 / \lambda_{\min \mathrm{A}}}+\sqrt{2 \eta_{\mathrm{o}}^{2} / \lambda_{\min }+8 \mathrm{~K} \eta_{\mathrm{o}} \varepsilon_{\mathrm{S}} / \lambda_{\max }+16 \mathrm{~K}^{2}\left(\sum_{i=1}^{6}\left(\bar{a}_{i}-a_{i}\right)^{2}\right) / \lambda_{\operatorname{maxA}}^{2}}}{4 \mathrm{~K} / \lambda_{\operatorname{maxA}}}\right) .
\end{gathered}
$$


The proposed robust detumbling controller with real-time estimation of bounds on the target's inertial parameters ensures that the end-effector's velocity will converge to a neighbourhood of $\left\|{ }^{\mathrm{e}} \boldsymbol{v}_{\mathrm{e}}\right\|=0$ and is ultimately upper bounded by (3.80). A reduction in the size of $\eta_{\mathrm{o}}$ will result in a reduction of the ultimate upper bound. This can be made possible with the selection of the gains $k_{i}$ and $c_{i}$ such that the ratio $k_{i} / c_{i}$ is reduced. Furthermore, a smaller selection of $\varepsilon_{\mathrm{r}}$ will also result in a reduce $\eta_{\mathrm{o}}$. However, this increases the likelihood of chattering and this trade off must be considered in its selection.

\subsubsection{Force/Torque Error}

Rearranging 3.49a) and utilizing 3.71), the components of the force/torque error, ${ }^{\mathrm{e}} \tilde{\mathbf{f}}_{\mathrm{e} i}={ }^{\mathrm{e}} \mathbf{f}_{\mathrm{e} i}-$ ${ }^{\mathrm{e}} \mathbf{f}_{\mathrm{eD} i}$ for $i=1,2,3 \ldots 6$, are upper bounded by:

$$
\left|{ }^{\mathrm{e}} \tilde{\mathbf{f}}_{\mathrm{e} i}\right| \leq \overline{\mathrm{D}}_{i}\left(1+2 \tanh \left(\frac{12 \overline{\mathrm{D}}_{i} \mathrm{k}_{\mathrm{u}}\left\{{ }^{\mathrm{e}} \boldsymbol{v}_{\mathrm{e}}\right\}_{i}}{\varepsilon_{\mathrm{r}}}\right)\right)
$$

where $\overline{\mathrm{D}}_{i}$ is computed as in 3.59 with the use of $\bar{a}_{i}$ instead of $a_{i}$.

From (3.81), the largest value of ${ }^{\mathrm{e}} \tilde{\mathbf{f}}_{\mathrm{e} i}$ is $3 \overline{\mathrm{D}}_{i}$ and decreases as the target's linear and angular velocities converge to a neighbourhood of zero. As per 3.59 , the value of $\overline{\mathrm{D}}_{i}$ is dependent on the target's size, rotation rate, inertia, contact force/torque and decreases as the target is detumbled. The rotation rate of a tumbling satellite can be assumed small, [40, 53, 63], and the contact force/torque scaled by the inertia of the target becomes negligible as both the contact force and torque are scaled by $\left(m_{\mathrm{tL}}^{-1}+r_{\mathrm{teU}}^{2} \lambda_{\operatorname{minL}}^{-1}\right), r_{\mathrm{teU}} \lambda_{\operatorname{minL}}^{-1}$ and $\lambda_{\operatorname{minL}}^{-1}$ in 3.59 .

\subsubsection{Implications of Servicer's Force/Torque Limits}

Adhering to end-effector force/torque limits is an important mission constraint in the postgrasping phase. Force/torque limits at the grasping point can be determined based on force/torque limits of the grasping mechanism at the servicer's end-effector. Exceeding these limits will result in damage of the grasping mechanism and potential lost of contact with the target. Additionally, end-effector force/torque limits are determined based on the servicer's attitude control system torque limits and the manipulator joint torque limits.

In order to establish a relationship between the servicer's force/torque limits at the grasping point and the maximum torque experienced by the servicer's manipulator joints and base attitude control system, it is first assumed that $\dot{\mathbf{w}}_{\mathrm{b}}=\mathbf{w}_{\mathrm{b}}=0$. This assumption reflects the study 
conducted by [8], where the pre-grasping phase intercept trajectory to grasp the target is designed to avoid impact with the target's grasping point. This will result in zero disturbance to the servicer's base. With this assumption, keeping the base undisturbed in the post-grasping phase will ensure that the target's momentum is rejected while its tumbling motion is brought to rest. The resultant manipulator joints and base attitude control system torque limits to be determined below, reflect the maximum torques required to achieve this while being subjected to an external force/torque at the end-effector in the post-grasping phase. Utilizing the above assumption in the servicer's equation of motion, 2.36), results in the following (servicer does not possess redundancy with respect to the detumbling task):

$$
\begin{gathered}
\tau_{\mathrm{m}}=\overline{\mathbf{M}}_{11} \dot{\boldsymbol{v}}_{\mathrm{e}}+\overline{\mathbf{c}}_{\mathrm{e}}+\overline{\mathbf{J}}_{\mathrm{e}} \mathbf{f}_{\mathrm{e}} \\
\tau_{\mathrm{b}}=\overline{\mathbf{M}}_{21} \dot{\boldsymbol{v}}_{\mathrm{e}}+\overline{\mathbf{c}}_{1}+\overline{\mathbf{J}}_{1} \mathbf{f}_{\mathrm{e}}
\end{gathered}
$$

where $\overline{\mathbf{M}}, \overline{\mathbf{c}}$ and $\overline{\mathbf{J}}$ are partitioned into the following components:

$$
\overline{\mathbf{M}}=\left[\begin{array}{ll}
\overline{\mathbf{M}}_{11} & \overline{\mathbf{M}}_{12} \\
\overline{\mathbf{M}}_{21} & \overline{\mathbf{M}}_{22}
\end{array}\right] \quad \overline{\mathbf{c}}=\left[\begin{array}{c}
\overline{\mathbf{c}}_{\mathrm{e}} \\
\overline{\mathbf{c}}_{1}
\end{array}\right] \quad \overline{\mathbf{J}}=\left[\begin{array}{c}
\overline{\mathbf{J}}_{\mathrm{e}} \\
\overline{\mathbf{J}}_{1}
\end{array}\right]
$$

where $\overline{\mathbf{M}}_{11} \in \mathbb{R}^{m \times 6}, \overline{\mathbf{M}}_{12} \in \mathbb{R}^{m \times 3}, \overline{\mathbf{M}}_{21} \in \mathbb{R}^{3 \times 6}, \overline{\mathbf{M}}_{22} \in \mathbb{R}^{3 \times 3}, \overline{\mathbf{c}}_{\mathrm{e}} \in \mathbb{R}^{m}, \overline{\mathbf{c}}_{1} \in \mathbb{R}^{3}, \overline{\mathbf{J}}_{\mathrm{e}} \in \mathbb{R}^{m \times 6}$ and $\overline{\mathbf{J}}_{1} \in \mathbb{R}^{3 \times 6}$.

From (3.82a) and (3.82b), in order to keep the servicer's base undisturbed (i.e. reject the target's gained momentum) it is required that the servicer's manipulator joint torques, $\tau_{\mathrm{m}}$, and servicer's base attitude control torques, $\tau_{\mathrm{b}}$, be equal to the terms on the right side of the equations. The end-effector's acceleration, $\dot{v}_{\mathrm{e}}$, can be eliminated from (3.82a) and (3.82b) with

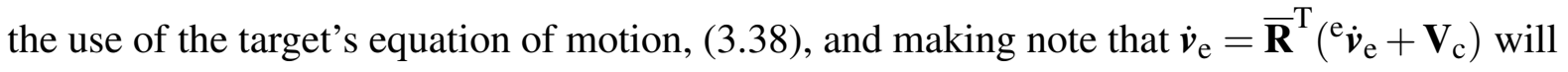
result in the following:

$$
\begin{aligned}
\tau_{\mathrm{m}} & =\left(\overline{\mathbf{M}}_{11} \overline{\mathbf{R}}^{\mathrm{T}} \Lambda_{\mathrm{te}}^{-1} \overline{\mathbf{R}}+\overline{\mathbf{J}}_{\mathrm{e}}\right) \mathbf{f}_{\mathrm{e}}+\left(\overline{\mathbf{c}}_{\mathrm{e}}+\overline{\mathbf{M}}_{11} \overline{\mathbf{R}}^{\mathrm{T}}\left(\mathbf{V}_{\mathrm{c}}-\Lambda_{\mathrm{te}}^{-1} \mathbf{J}_{\mathrm{te}}^{-\mathrm{T}} \mathbf{c}_{\mathrm{te}}\right)\right) \\
& \approx\left(\overline{\mathbf{M}}_{11} \overline{\mathbf{R}}^{\mathrm{T}} \Lambda_{\mathrm{te}}^{-1} \overline{\mathbf{R}}+\overline{\mathbf{J}}_{\mathrm{e}}\right) \mathbf{f}_{\mathrm{e}} \\
\tau_{\mathrm{b}} & =\left(\overline{\mathbf{M}}_{21} \overline{\mathbf{R}}^{\mathrm{T}} \Lambda_{\mathrm{te}}^{-1} \overline{\mathbf{R}}+\overline{\mathbf{J}}_{1}\right) \mathbf{f}_{\mathrm{e}}+\left(\overline{\mathbf{c}}_{1}+\overline{\mathbf{M}}_{21} \overline{\mathbf{R}}^{\mathrm{T}}\left(\mathbf{V}_{\mathrm{c}}-\Lambda_{\mathrm{te}}^{-1} \mathbf{J}_{\mathrm{te}}^{-\mathrm{T}} \mathbf{c}_{\mathrm{te}}\right)\right) \\
& \approx\left(\overline{\mathbf{M}}_{21} \overline{\mathbf{R}}^{\mathrm{T}} \Lambda_{\mathrm{te}}^{-1} \overline{\mathbf{R}}+\overline{\mathbf{J}}_{1}\right) \mathbf{f}_{\mathrm{e}}
\end{aligned}
$$


In (3.83a and (3.83b), it was approximated that the non-linear velocity dependent terms are negligible due to the slow rotation rate of the target [40, 53, 63]. From (3.83a) and 3.83b), the manipulator and base attitude control torques are upper bounded as follows:

$$
\begin{gathered}
\left\|\tau_{\mathrm{m}}\right\| \leq\left(\left\|\overline{\mathbf{M}}_{11}\right\|\left\|\Lambda_{\mathrm{te}}^{-1}\right\|+\left\|\overline{\mathbf{J}}_{\mathrm{e}}\right\|\right) \mathrm{K}_{\max } \\
\left\|\tau_{\mathrm{b}}\right\| \leq\left(\left\|\overline{\mathbf{M}}_{21}\right\|\left\|\Lambda_{\text {te }}^{-1}\right\|+\left\|\overline{\mathbf{J}}_{1}\right\|\right) \mathrm{K}_{\max }
\end{gathered}
$$

where $\|\overline{\mathbf{R}}\|=1$ and $\mathrm{K}_{\max }=\max \left\{\mathrm{F}_{\max }, \tau_{\max }\right\}$. The upper bound of $\mathbf{f}_{\mathrm{e}}$ is the maximum of the force and torque limit at the end-effector and is denoted as $\mathrm{K}_{\max }$. From (3.84a) and 3.84b), increasing the force/torque limits at the end-effector will result in an increase of the maximum manipulator torque or maximum servicer's attitude control torque required to keep the servicer's base undisturbed and reject the target's gained momentum. The norms $\left\|\overline{\mathbf{M}}_{11}\right\|,\left\|\overline{\mathbf{M}}_{21}\right\|$, $\left\|\overline{\mathbf{J}}_{\mathrm{e}}\right\|$ and $\left\|\overline{\mathbf{J}}_{1}\right\|$ are dependent on the known servicer's inertial parameters and geometry, and their largest possible values can be determined. The target's inertial parameters are unknown, but the largest possible value of $\left\|\Lambda_{\mathrm{te}}^{-1}\right\|$ can be determined based on pre-launched data of the target. For target's with sufficiently large inertia, the first terms of the upper bounds of $\tau_{\mathrm{m}}$ and $\tau_{\mathrm{b}}$ can be approximated as zero $\left(\left\|\overline{\mathbf{M}}_{11}\right\|\left\|\Lambda_{\mathrm{te}}^{-1}\right\|=\left\|\overline{\mathbf{M}}_{21}\right\|\left\|\Lambda_{\mathrm{te}}^{-1}\right\| \approx 0\right)$ so that the above inequalities in (3.84) reduces to:

$$
\begin{gathered}
\left\|\tau_{\mathrm{m}}\right\| \leq\left\|\overline{\mathbf{J}}_{\mathrm{e}}\right\| \mathrm{K}_{\max } \\
\left\|\tau_{\mathrm{b}}\right\| \leq\left\|\overline{\mathbf{J}}_{1}\right\| \mathrm{K}_{\max }
\end{gathered}
$$

\subsubsection{Numerical Simulation}

This section presents the evaluation of the proposed detumbling controller to detumble a noncooperative target with unknown but bounded inertial parameters, while being subjected to force/torque limits at the servicer's end-effector. The evaluation is carried out using a 7-degreeof-freedom (DOF) space manipulator servicer satellite that is based on a modified model of ETS-VII system. The model parameters of the servicer are described in Appendix A. The target is modelled as a cube with $1.95 \mathrm{~m}$ sides and its inertia parameters are presented in 
Table 3.1: Target Properties for Numerical Simulation Studies

\begin{tabular}{ccc} 
Condition: & Dry & Wet \\
\hline$m_{\mathrm{t}}, \mathrm{kg}$ & 350 & 500 \\
$\mathbf{I}_{\mathrm{t}}, \mathrm{kgm}^{2}$ & $\operatorname{diag}([212.8,212.8,219.9])$ & $\operatorname{diag}([263.4,263.4,240.1])$ \\
$\left\|\mathbf{r}_{\mathrm{te}}\right\|, \mathrm{m}$ & 1.62 & 1.71 \\
\hline
\end{tabular}

Table 3.1. Further details on the modelling of the target and its inertial parameters can be found in Appendix A. For the numerical simulation, the target will be modelled with zero fuel and its inertial parameters will reflect that of the Dry Condition in Table 3.1.

The target is grasped at $t=0 \mathrm{~s}$, resulting in a redistribution of its momentum in the servicertarget system. The velocities of the servicer-target system after grasping $\left(t^{+}=0 \mathrm{~s}\right)$ are computed as in [67]. The target has an initial angular velocity of $\mathbf{w}_{\mathrm{t}}=[-3.9,-3.9,-6.5]^{\mathrm{T}} \mathrm{deg} / \mathrm{s}$ at the start of the post grasping phase. The initial velocity of the target in the post-grasping phase is reasonable as ground based observation of ADEOS 1 described its attitude motion as composed of two rotational components: $0.1 \mathrm{deg} / \mathrm{s}$ about the satellite's main body and another about the satellite's boom of about $0.4 \mathrm{deg} / \mathrm{s}$ [77]. Observation of Envisat resulted in an average rotation rate of 3.5deg/s [62]. Functions from the SpaceDyn toolbox were utilized to compute the servicer's mass matrix, Jacobian and nonlinear forces [69].

The manipulator and base attitude control torques, $\tau_{\mathrm{m}}$ and $\tau_{\mathrm{b}}$, are defined as per (3.1). Control of the servicer's base and end-effector are defined by the auxiliary inputs, $\mathbf{u}_{\mathrm{f}}$ in (3.41) and $\mathbf{u}_{\mathrm{b}}$ in 3.3a), respectively. The reference force/torque is defined in 3.39 3.40). The robust compensator, $\Psi$, is defined in (3.71) and estimates of the bounds on the target's inertial parameters, $\hat{a}_{i}$ for $i=1,2,3, \ldots, 6$, are updated as per 3.72 .

The proposed detumbling strategy is presented for two different cases. Both cases utilize the same controller gains. In Case 1, the servicer's base control torques are realized with continuous torques applied to its center of mass. In Case 2, the servicer's base control torques are achieved with reaction thrusters that provide only on-off thrust. Case 2 is presented to demonstrate that the proposed detumbling strategy can also be incorporated with a pulse-width pulse-frequency (PWPF) modulator to control the servicer's base using on-off thrusters located on the servicer's base. From Fig. 3.7, the PWPF modulator is a feedback loop containing a linear first-order filter and Schmidt trigger and are characterized by the user-defined positive parameters: $\mathrm{K}_{m}, \mathrm{~T}_{m}, \mathrm{U}_{\text {on }}, \mathrm{U}_{\text {off }}$ and $\mathrm{Y}_{\max }$ [25, 50]. Let $\Sigma_{\mathrm{b}}$ denote 
the body fixed frame located at the servicer's base center of mass. The components of the base attitude control torque in $\Sigma_{\mathrm{b}}$ are inputs to the PWPF modulator. The outputs of the PWPF modulator are used to fire a set of thrusters based on the thruster configuration in Fig. 3.8 and the thruster selection logic in Table 3.2 in order to apply a torque to the servicer's base. The thrusters are assumed to have a specific impulse of $I_{s p}=70 \mathrm{~s}$ and generate an on-off reaction force of $8 \mathrm{~N}$. This is a reasonable value for the reaction force, as thrusters with similar values have been utilized with orbiting satellites [36]. Furthermore,

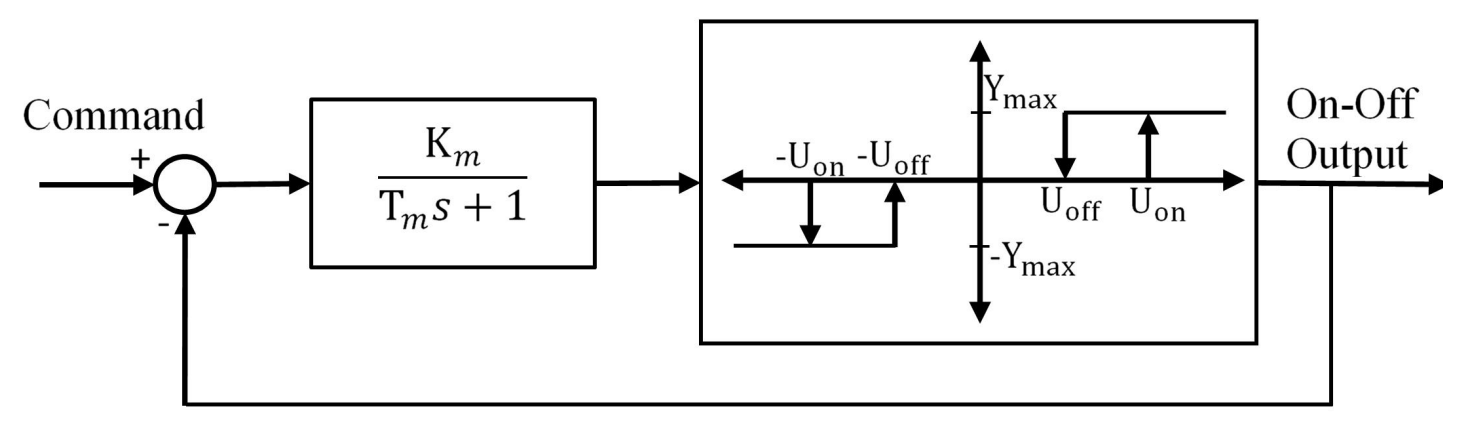

Figure 3.7: Block diagram of the PWPF modulator.

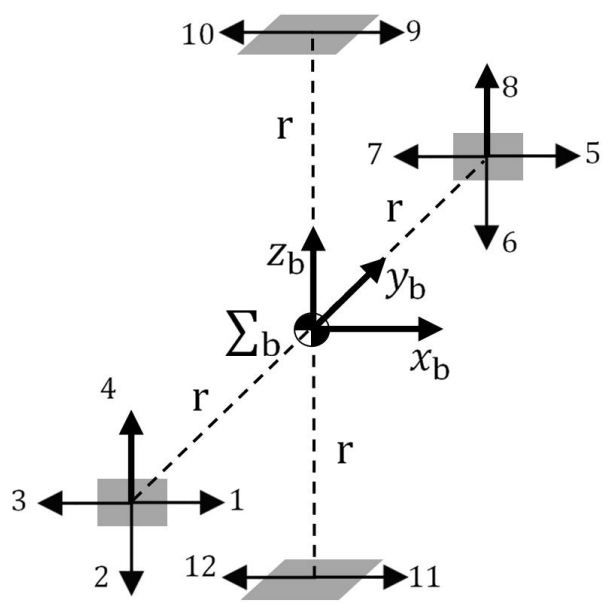

Figure 3.8: Servicer's base thruster configuration. $\Sigma_{\mathrm{b}}$ denotes body fixed frame located at servicer's base center of mass and coincides with principle axis. $r=1.15 \mathrm{~m}$ 
Table 3.2: Thruster Firing Logic

\begin{tabular}{ccc}
\hline \multicolumn{2}{c}{ PWPF modulator } & \\
\cline { 1 - 2 } Input & Output & Thruster turned ON \\
\hline${ }^{\mathrm{b}} \tau_{\mathrm{bx}}$ & $\mathrm{Y}_{\max }$ & $\# 4, \# 6$ \\
${ }^{\mathrm{b}} \tau_{\mathrm{bx}}$ & $-\mathrm{Y}_{\max }$ & $\# 2, \# 8$ \\
${ }^{\mathrm{b}} \tau_{\mathrm{by}}$ & $\mathrm{Y}_{\max }$ & $\# 10, \# 11$ \\
${ }^{\mathrm{b}} \tau_{\mathrm{by}}$ & $-\mathrm{Y}_{\max }$ & $\# 9, \# 12$ \\
${ }^{\mathrm{b}} \tau_{\mathrm{bz}}$ & $\mathrm{Y}_{\max }$ & $\# 3, \# 5$ \\
${ }^{\mathrm{b}} \tau_{\mathrm{bz}}$ & $-\mathrm{Y}_{\max }$ & $\# 1, \# 7$ \\
\hline
\end{tabular}

this produces a torque of $18.4 \mathrm{Nm}$ about the servicer's base center of mass which is reasonable as reaction torque levels ranging between $0.01 \mathrm{Nm}$ and $30 \mathrm{Nm}$ are common in most spacecraft using reaction thrusters for attitude control [56].

As per the stated assumptions in Chapter 2, it is assumed that inertial measurements can be obtained from an inertial observer at a sample rate of $0.01 \mathrm{~s}$. In both cases, the servicer is modelled with $90 \%$ of its true geometry and inertia, and neglects compensation of $\overline{\mathbf{c}}$ in (3.1). Furthermore, joint null space damping is utilized to minimize excess manipulator joint motion in the null space of the detumbling task at the servicer's end-effector. The controller gains for both Cases 1 and 2 are as follows: $\mathbf{K}_{\mathrm{AD}}=1.5 \mathbf{E}_{3}, \mathbf{K}_{\mathrm{fP}}=0.2 \mathbf{E}_{6}, \varepsilon_{\mathrm{r}}=4, \mathrm{~F}_{\max }=10 \mathrm{~N}, \tau_{\max }=$ $10 \mathrm{Nm}$. The PWPF modulator parameters are $\mathrm{K}_{m}=2, \mathrm{~T}_{m}=0.8, \mathrm{U}_{\mathrm{on}}=0.1, \mathrm{U}_{\mathrm{off}}=0.6 \mathrm{U}_{\mathrm{on}}$ and $\mathrm{Y}_{\max }=12$. For the update law in (3.72), $\hat{a}_{i}(t=0)=\underline{a}_{i}$, the coefficient $\kappa_{i}=1$ and $c_{i}$ is selected as the $i_{\text {th }}$ element of $[1,1,0.25,1,0.25,0.1]$ for $i=1,2,3, \ldots, 6$. The upper and lower bounds on the target's inertial parameters are obtained as described in Appendix A (Table A.5 in Appendix A. From these bounds, the upper and lower bounds of the coefficients $a_{i}$ can be determined making use of 3.60 .

Regarding the selection of $\varepsilon_{\mathrm{s}}$ in the reference force/torque, 3.39 3.40), the following should be considered: a small $\varepsilon_{\mathrm{s}}$ will result in larger desired force/torque and hence faster detumbling of the target. While this is desirable, faster detumbling will result in larger endeffector acceleration and hence a larger rate of change in the desired force/torque as the endeffector's linear and angular velocities will converge to zero faster. Attempting to follow a fast changing desired force/torque may result in instability due to unmodeled dynamics. A large value for $\varepsilon_{\mathrm{s}}$ will result in a slow changing desired force/torque, and will lead to approx- 

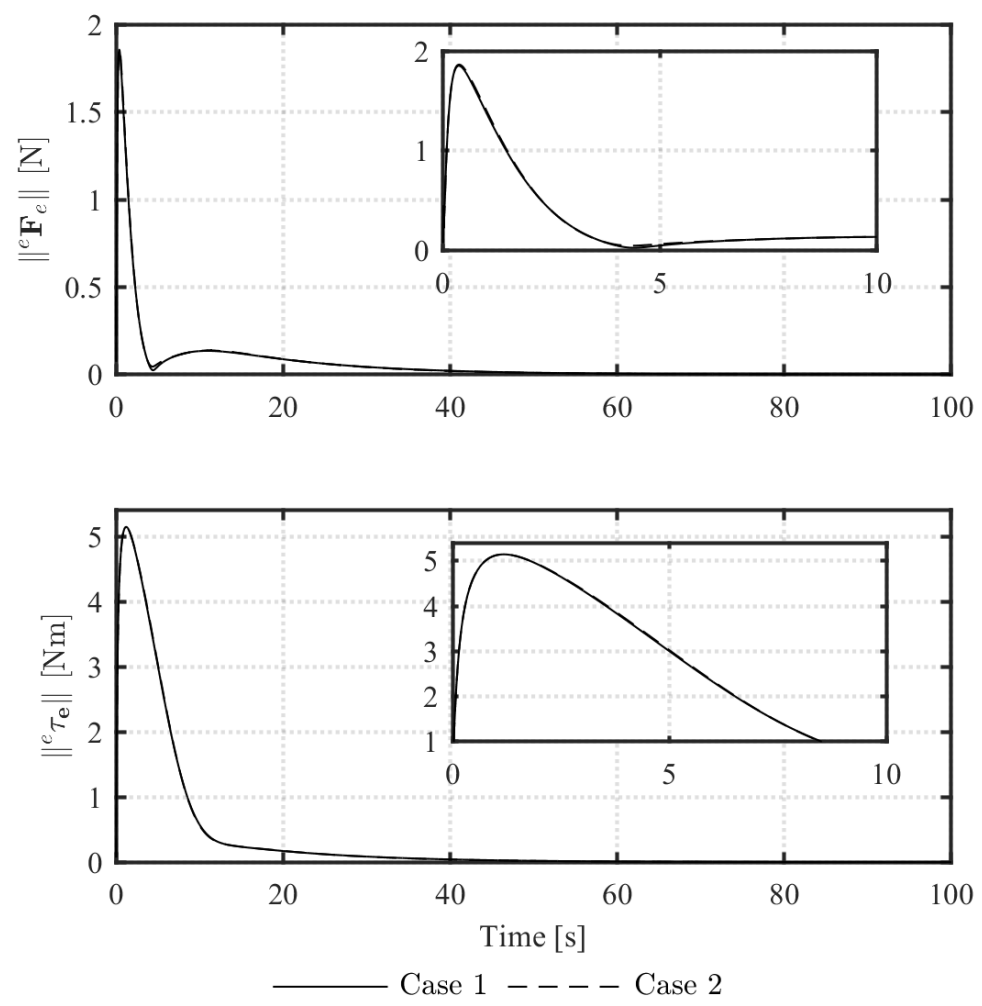

Figure 3.9: Magnitude of end-effector's force/torque profile for Cases 1 and 2.

imately zero desired force/torque as the end-effector's linear and angular velocities converge to zero. This will result in a prolonged detumbling period. To avoid this, $\varepsilon_{\mathrm{s}}$ is defined as: $\varepsilon_{\mathrm{s}}=2.5\left\|{ }^{\mathrm{e}} v_{\mathrm{e}}\right\|+0.1$ for both Cases 1 and 2 .

The simulation results are reported in Figs. 3.9-3.15 for both Cases 1 and 2. It can be observed in Fig. 3.9 that in both cases the end-effector's force/torque limit is respected. Detumbling of the target is can be observed to have been accomplished as the servicer's end-effector's velocity converge to a neighbourhood of zero in Fig. 3.10 .

As the target is brought to rest, its momentum is transferred to the servicer and must be rejected in order to avoid tumbling of the combined servicer/target system. The maximum expected torque of the manipulator and base attitude control system required to reject the gained target's momentum are determined using inequality (3.85) to be $19.5 \mathrm{Nm}$ and $17.9 \mathrm{Nm}$, respectively. The maximum manipulator and base attitude control torque from Fig. 3.11 are observed to be within these bounds. Note, from (3.85), the maximum manipulator and base attitude control system torques are directly proportional to the force/torque limits at the end-effector. 

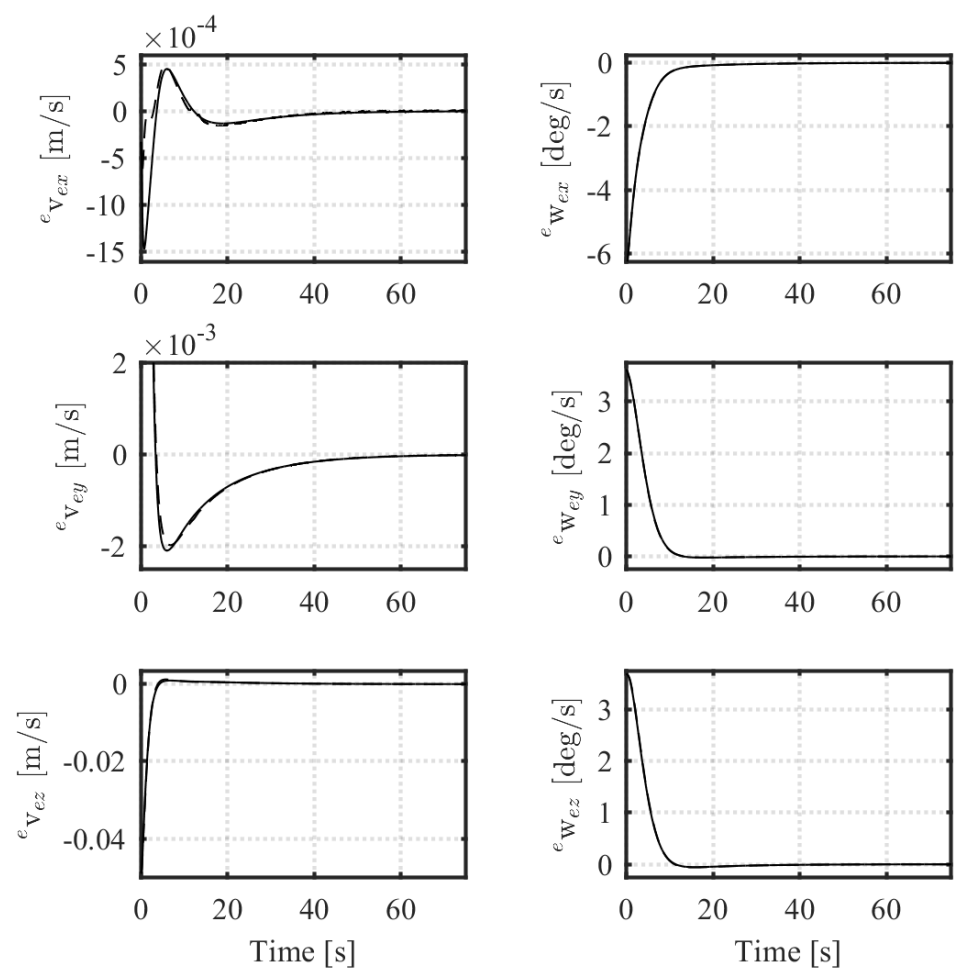

Figure 3.10: End-effector's linear and angular velocities for Cases 1 and 2.
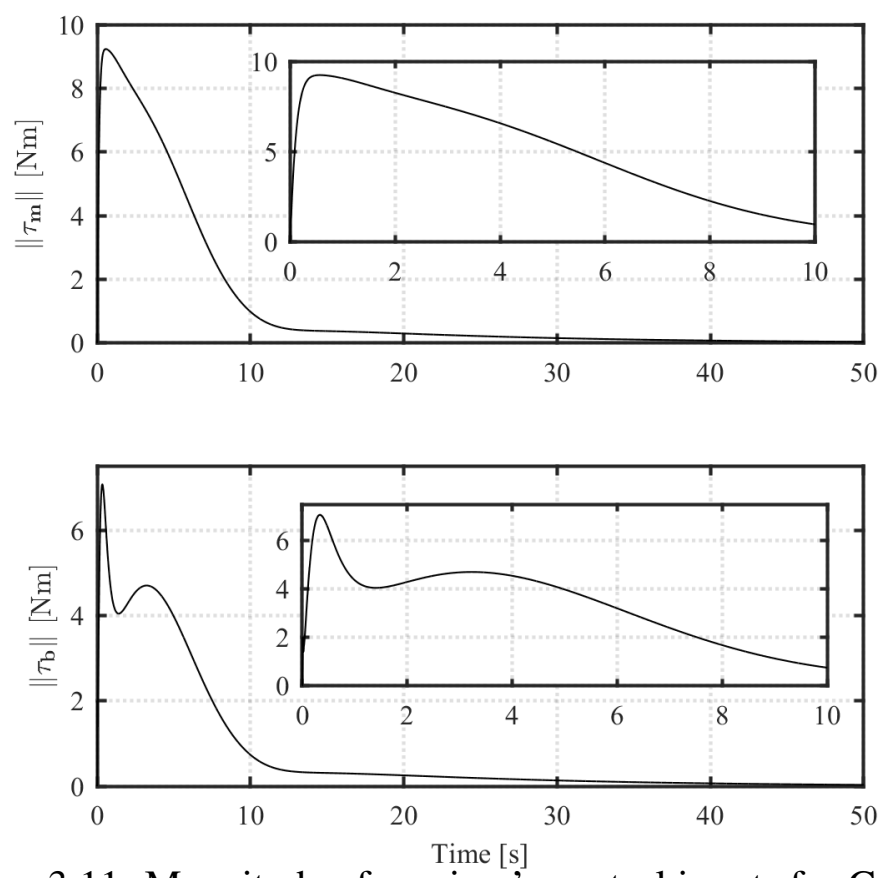

Figure 3.11: Magnitude of servicer's control inputs for Case 1. 

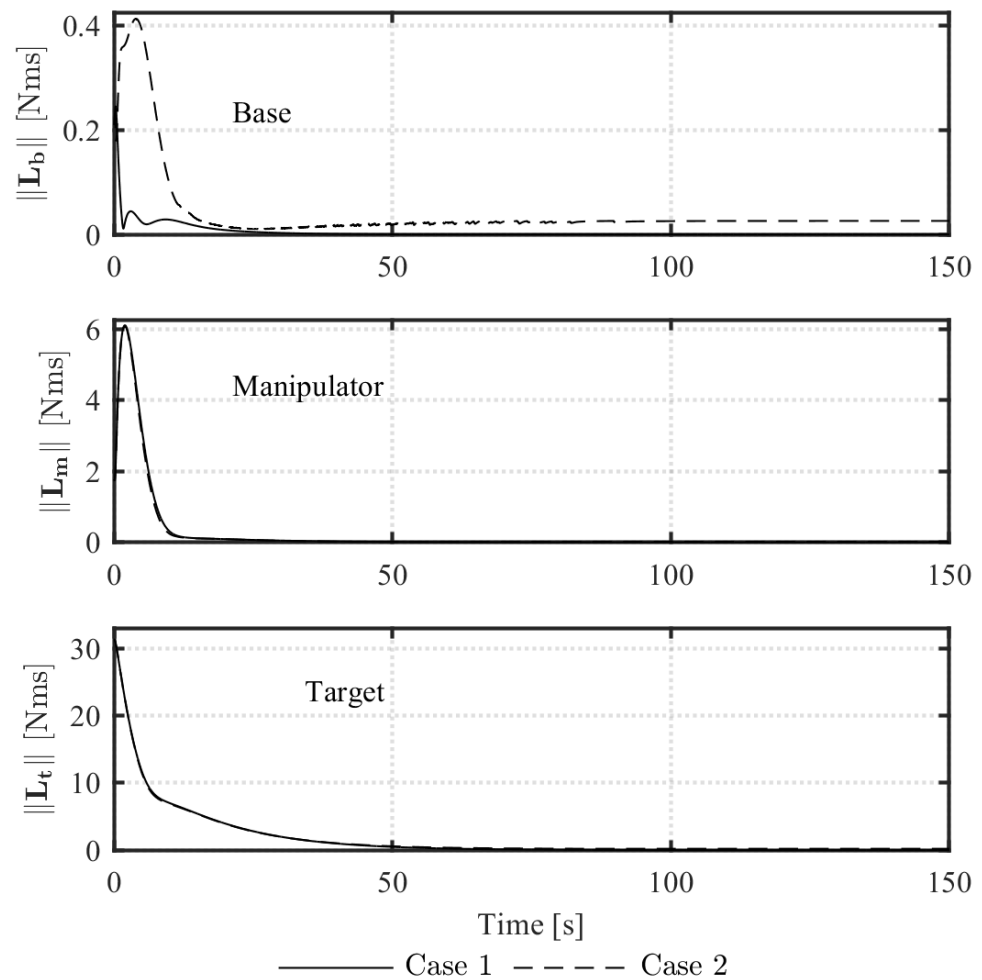

Figure 3.12: Servicer-target system's momentum distribution for Cases 1 and 2.

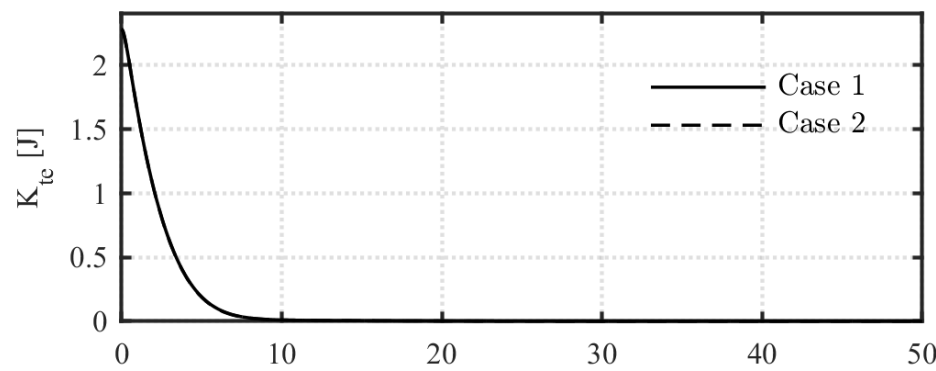

Figure 3.13: Target's kineticienergy for Cases 1 and 2.

Larger end-effector force/torque implies that the target's momentum is being transferred faster to the servicer and needs to rejected at a larger rate in order to avoid tumbling of the combined system. Rejection of the target's momentum is observed in Fig. 3.12 as the servicer's and target's momentum converge to a neighbourhood of zero. Furthermore, as per the stability analysis, the target's kinetic energy is presented in Fig. 3.13 and is observed to monotonically converge to a neighbourhood of zero. 

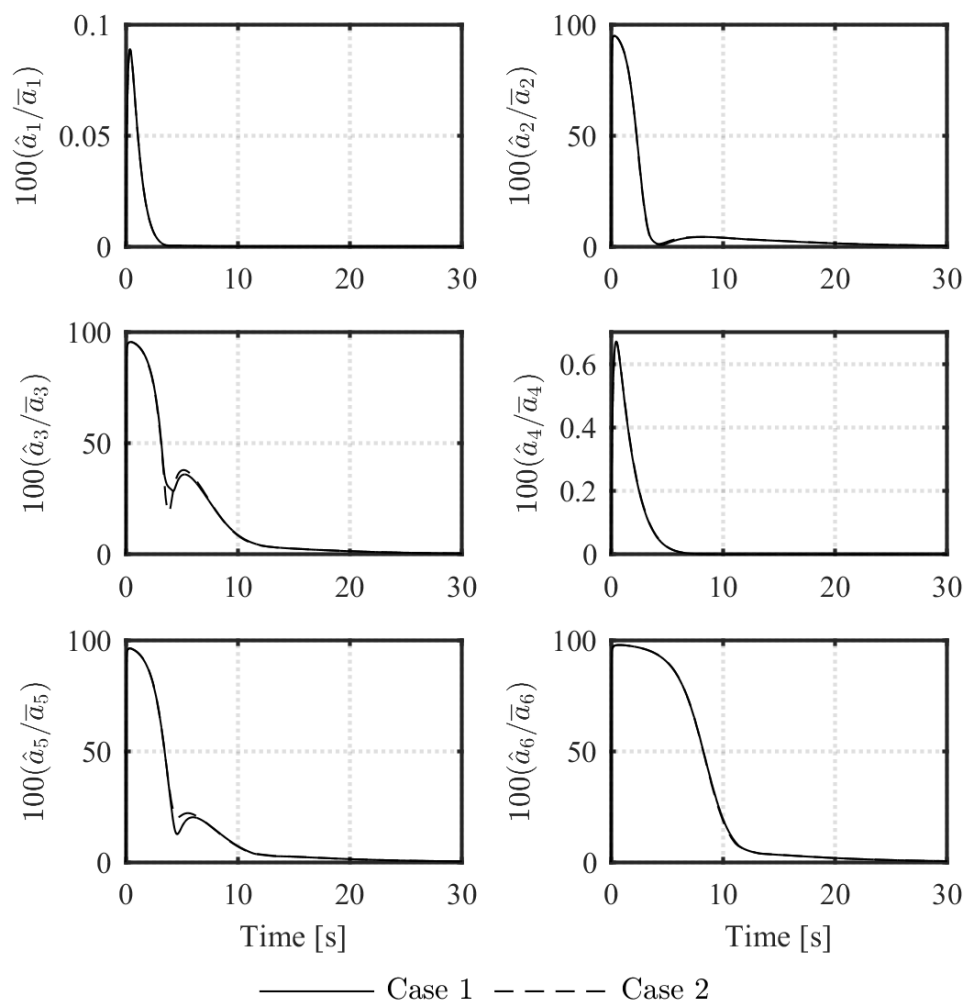

Figure 3.14: Time history of $\hat{a}_{i}$ as a percentage of $\bar{a}_{i}$ for Cases 1 and 2.

For both Cases 1 and 2, the estimated bounds $\left(\hat{a}_{i}\right)$ are presented in Fig. 3.14 as a percentage of their maximum value $\left(\bar{a}_{i}\right)$. From Fig. 3.14, it can be observed that some of these values are significantly less than $\bar{a}_{i}$ for the duration of the detumbling period and results in the reduced need of high robust gains (high robust gains are computed using $\bar{a}_{i}$ ). This highlights the benefit of estimating these parameters during the detumbling procedure as the controller will determine the required values of these parameters in order to detumble the target and in most cases the largest possible values of these parameters are not required as observed in Fig. 3.14.

The servicer's base torque output for Case 2 is presented in Fig. 3.15. According to the classical rocket equation the rate of change of the spacecraft's mass is determined by: $\dot{m}=$ $\left\|\mathbf{F}_{\mathrm{c}}\right\| /\left(I_{\mathrm{sp}} g_{\mathrm{r}}\right)$ where $\mathbf{F}_{\mathrm{c}}$ is the thruster force vector of the reaction thruster, $I_{s p}$ is the thruster's specific impulse, and $g_{\mathrm{r}}=9.81 \mathrm{~m} / \mathrm{s}^{2}$. Using this equation, the fuel consumption in Case 2 is $0.0868 \mathrm{~kg}$.

The above results demonstrate that the detumbling controller can detumble a target with unknown inertial parameters while being subjected to force/torque limits at the servicer's end- 

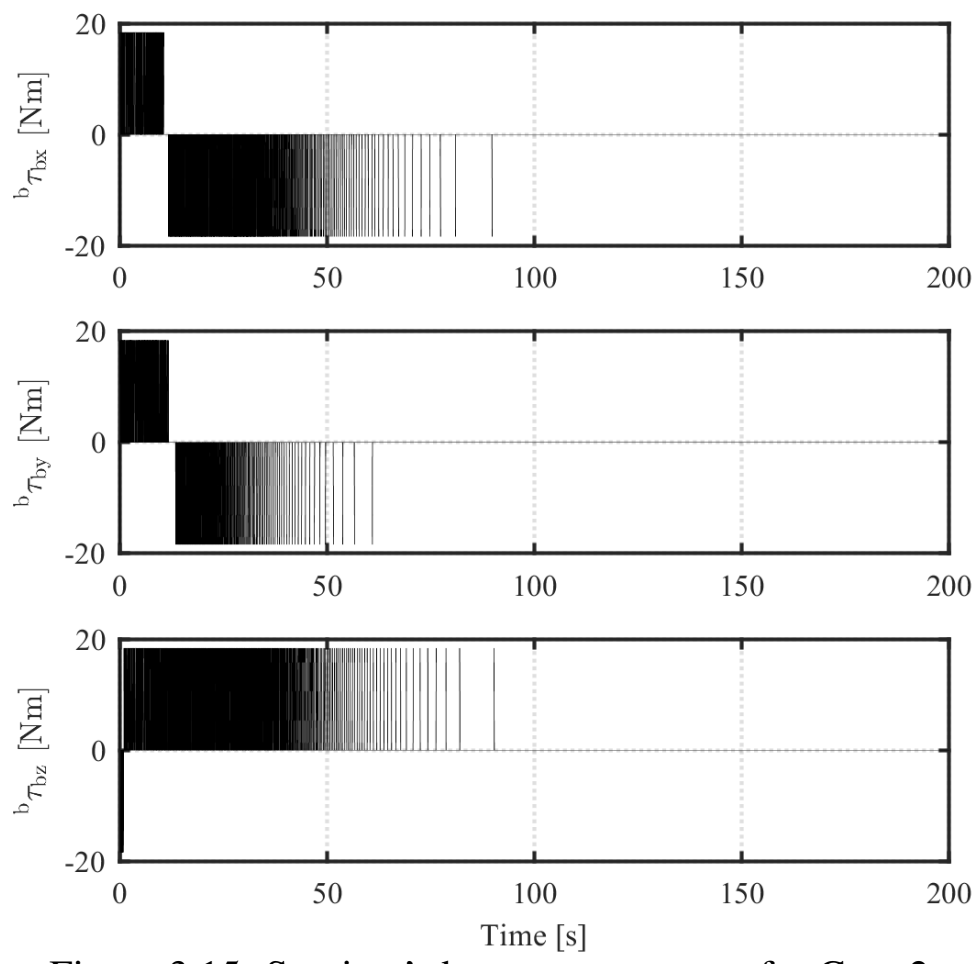

Figure 3.15: Servicer's base torque output for Case 2.

effector, even with the use of reaction thrusters to realized the control torques on the servicer's base as in Case 2. Furthermore, they indicate that knowledge of the target's inertial parameters are not required prior to post-grasping phase, and eliminates the need to perform parameter identification prior to the post-grasping phase in an attempt to identify the target's inertial parameters. Implementation of the detumbling controller requires conservative estimates $\left(\bar{a}_{i}\right)$ of bounds on the target's inertial parameters and can be obtained using pre-launched data of the target. In the presented numerical study, bounds (lower and upper) on the target's inertial parameters were based on the target's satellite inertial properties with and without fuel (wet and dry mass as described in Appendix A.

\subsubsection{Detumbling Target with Random Inertial Properties}

Let the target's dry mass, $m_{\text {dry }}$, be defined as $m_{\text {dry }}=350 \mathrm{~kg}$ as per Table 3.1 and denote the target's mass, $m_{\mathrm{t}}$, as the sum of its dry mass and mass of its fuel, $m_{\text {fuel }}$. From Table 3.1, the maximum fuel mass that the target can carry is $150 \mathrm{~kg}$. As the target's fuel mass varies from $0 \mathrm{~kg}$ and $150 \mathrm{~kg}$, so to does its inertial properties as described in Appendix A. 

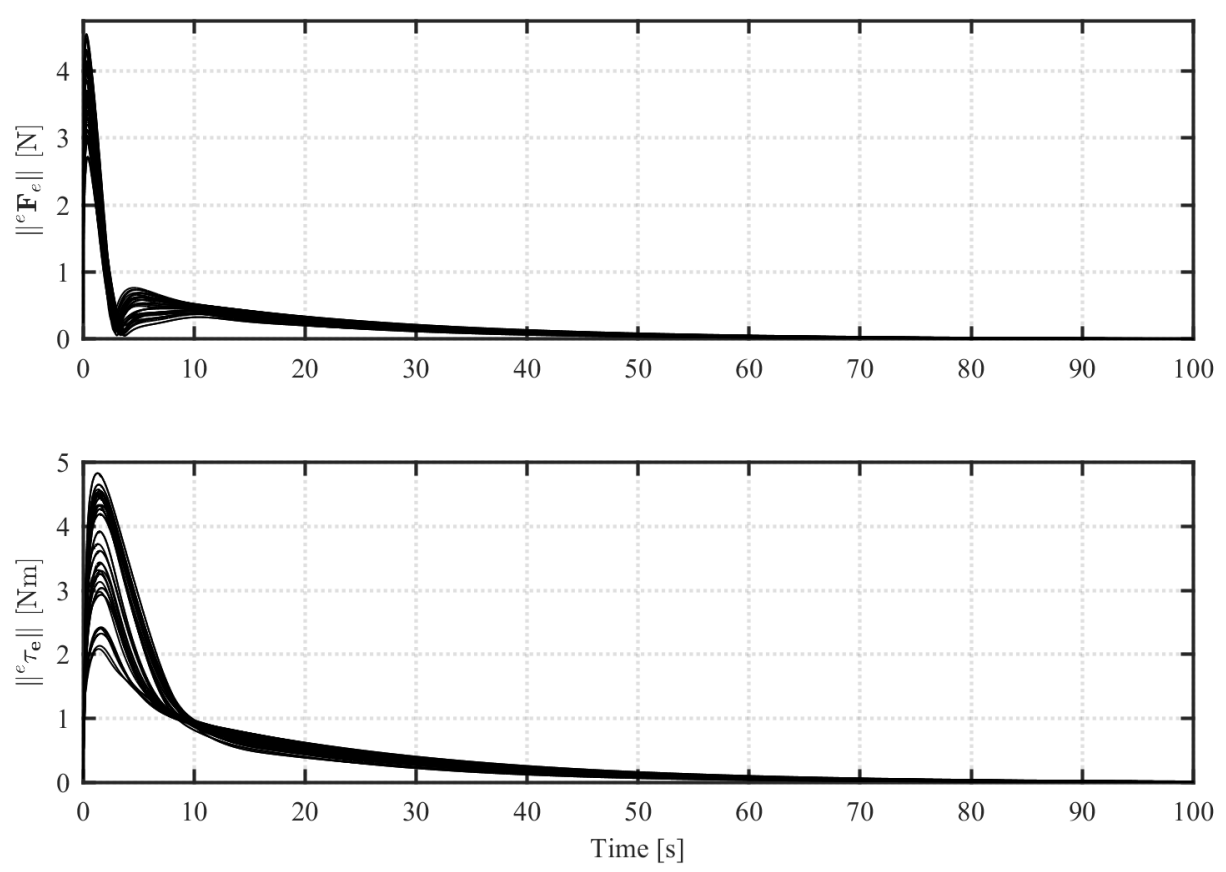

Figure 3.16: Magnitude of end-effector's force/torque profile as the target's fuel mass varies.

The purpose of the numerical example presented herein is to demonstrate the robustness of the proposed detumbling strategy in detumbling a target with unknown inertial parameters with known bounds. To this end, 50 simulations are performed, where $m_{\text {fuel }}$ is randomly selected as a value between $0 \mathrm{~kg}$ and $150 \mathrm{~kg}$. The target's initial angular velocity is assumed to have the same magnitude as that in Case 1 of Section 3.4.7, however the direction is randomly determined by a sequence of rotations as follows:

$$
\mathbf{w}_{\mathrm{tdir}}=\mathbf{R}_{z}\left(\theta_{1}\right) \mathbf{R}_{y}\left(\theta_{2}\right) \mathbf{R}_{x}\left(\theta_{3}\right)[0,0,1]^{\mathrm{T}}
$$

where $\mathbf{R}_{(\cdot)} \in \mathbb{R}^{3 \times 3}$ describes a rotation matrix about axis $(\cdot)=x, y, z$ by a randomly selected angle between $30 \mathrm{deg}$ and $-30 \mathrm{deg}$ for $\theta_{1}, \theta_{2}$ and $\theta_{3}$. This is accomplished with MATLAB's "rand" function. The control gains are the same as in Case 1 in Section 3.4.7 (servicer's base control torques are continuous).

The main results are reported in Figs. 3.16 and 3.17. From these results, the end-effector's force/torque limits are observed to be respected, Fig. 3.16, and the target's momentum is rejected as the angular momentum of the servicer and target all converge to a neighbourhood of zero as observed in Fig. 3.17 . 

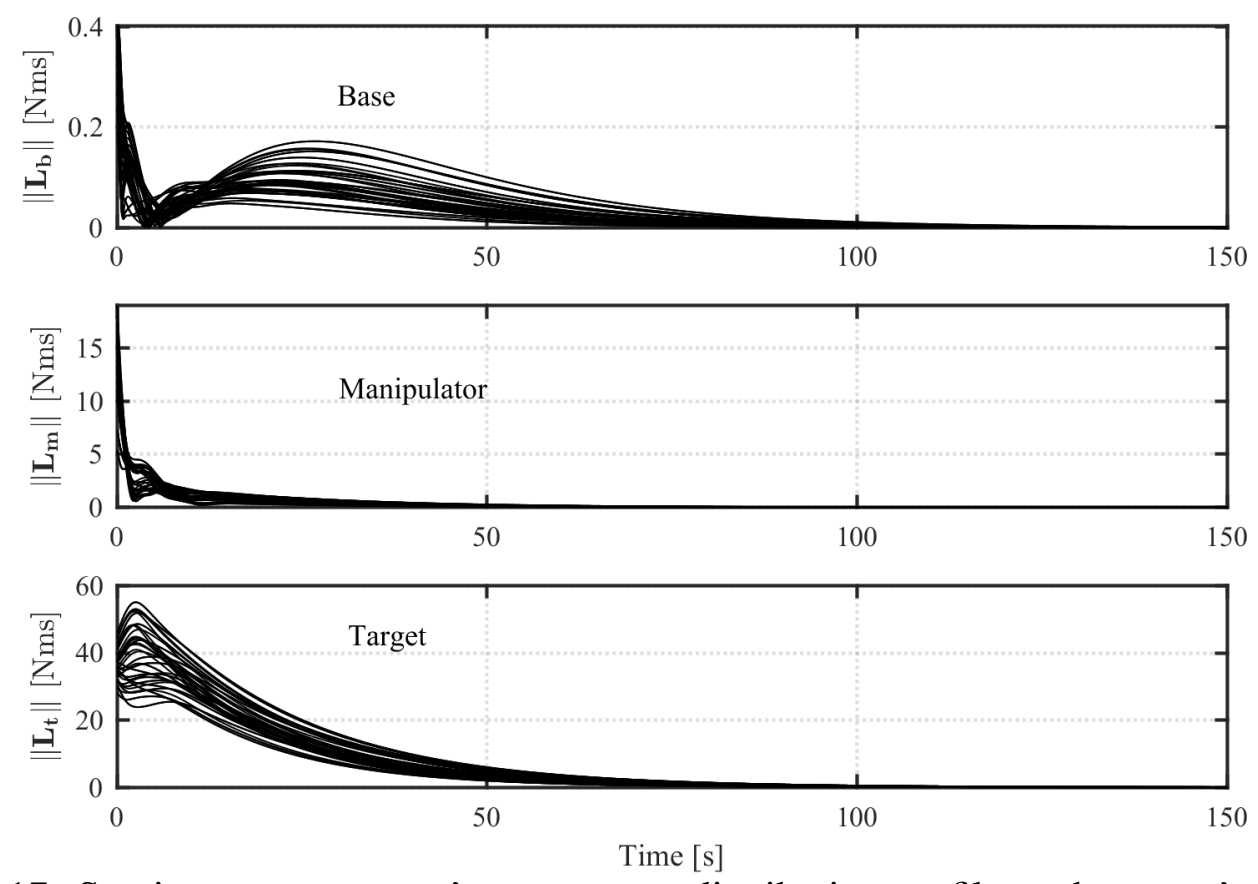

Figure 3.17: Servicer-target system's momentum distribution profile as the target's fuel mass varies.

\subsubsection{Effect of $\varepsilon_{\mathrm{r}}$ Parameter on Detumbling Controller's Performance}

The robust detumbling controller presented in Chapter 3.4 is a sliding mode controller, where the sliding variable can be regarded as $\mathrm{s}=\boldsymbol{v}_{\mathrm{e}}-\boldsymbol{v}_{\mathrm{e}}^{\mathrm{d}}$ and $\boldsymbol{v}_{\mathrm{e}}^{\mathrm{d}}=0$. The robust compensator presented in 3.50a is designed to force the system dynamics onto the sliding surface. The robust compensator could have been designed using a non-smooth function (i.e., sign function) [57]. The use of a non-smooth function can lead to chattering in practical implementation of sliding mode control due to the difficulty of switching the control law at high frequency in order to keep system's dynamics on the sliding surface, and the difficulty of avoiding the excitation of unmodeled dynamics when switching the controller at high frequency [58]. Hence the smooth robust compensator in 3.50a was utilized with the goal of avoiding this problem. This, however, requires the selection of the tuning parameter $\varepsilon_{\mathrm{r}}$ in 3.50a).

The influence of $\varepsilon_{\mathrm{r}}$ on the end-effector's force/torque profile and the servicer's control inputs are presented in Figs. 3.18 and 3.19 . Note, the same controller gains and initial conditions for the post-grasping phase as in Case 1 of Chapter 3.4.7 were utilized to obtain the results in Figs. 3.18 and 3.19. It can be observed in Fig. 3.18, that a decrease in $\varepsilon_{\mathrm{r}}$ results in an increase 

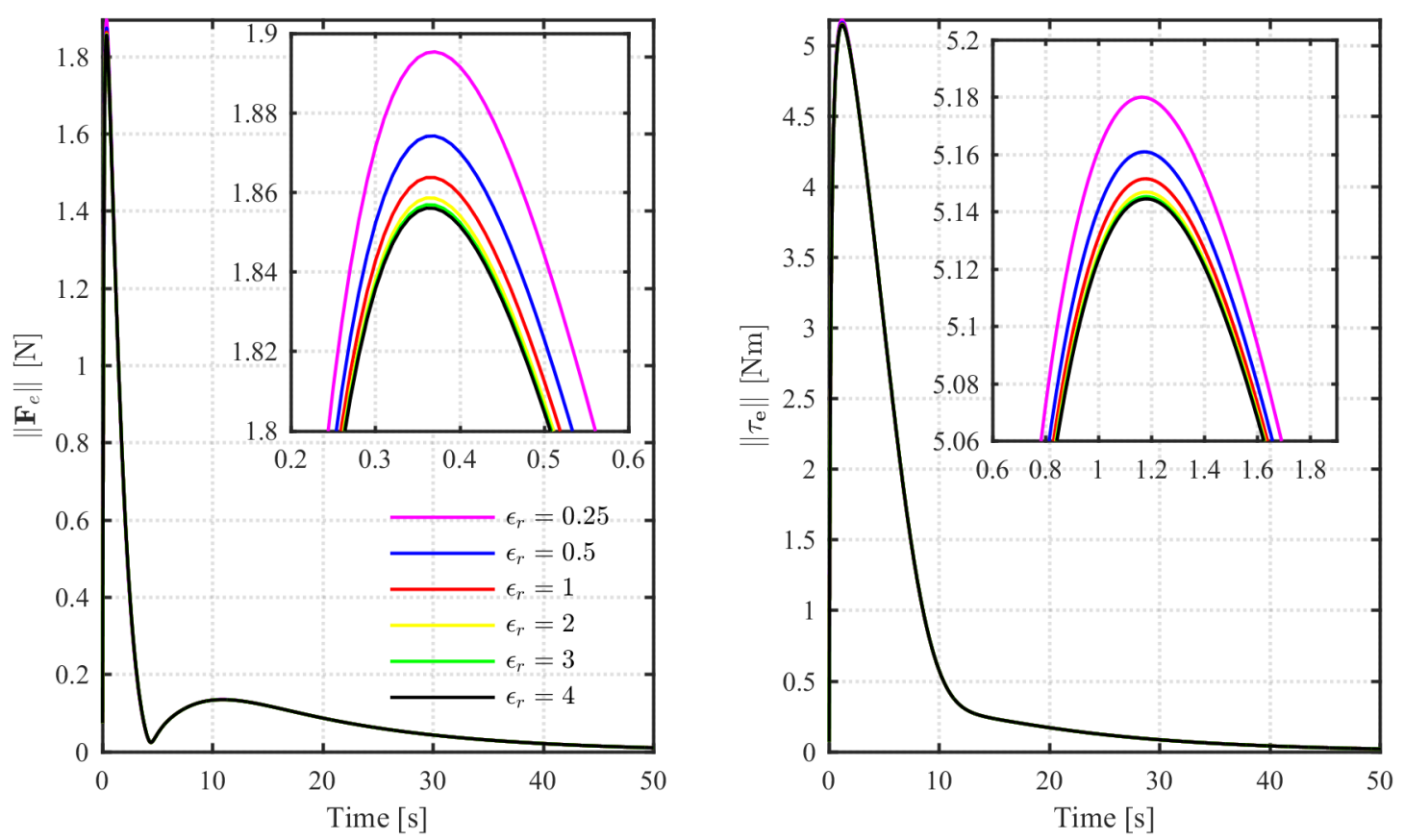

Figure 3.18: Effect of $\varepsilon_{\mathrm{r}}$ in $\Psi$ 3.50a on the end-effector force and torque magnitude profile in the post-grasping phase.

in end-effector's force and torque magnitude. Similar observation can be made regarding the servicer's control inputs in Fig. 3.19. This trend is to be expected. As the value of $\varepsilon_{\mathrm{r}}$ becomes smaller, the robust compensator produces a larger output for smaller inputs, resulting in larger value for the robust compensator. This in turn will result in larger servicer control inputs.

\subsection{Conclusions}

This chapter has addressed the problem of detumbling a non-cooperative target with unknown, but bounded inertial parameters by a space robot, which is subjected to force/torque limits at its end-effector. The detumbling strategy presented within this chapter was developed under the assumption that measurements of end-effector force/torque are available. Detumbling of the target is achieved by following a reference detumbling force/torque that is designed to bring the target's linear and angular velocity to zero subjected to force/torque limits at the servicer's endeffector. The reference detumbling force/torque is computed using the servicer's end-effector linear and angular velocity and force/torque limits. An adaptive robust compensator is designed to ensure stable detumbling of the target utilizing real-time estimates of bounds on the target's 
inertial parameters to update its gains. The design of the reference detumbling force/torque and use of real-time estimates of bounds on the target's inertial parameters eliminates the need for accurate knowledge of the target's inertial parameters and their bounds. The resultant detumbling controller can detumble the unknown target by damping its tumbling motion while allowing compliance with its residual unknown motion. Finally, numerical simulations have been conducted for a 7-degree-of-freedom space manipulator and have demonstrated the effectiveness of the proposed detumbling controller. This study lacked the means to experimentally evaluate the proposed detumbling strategy, and future work will aim to develop an experimental test platform. The experimental test platform will be used to evaluate the detumbling strategy developed in this chapter and to further advance research into post-grasping phase detumbling strategies.
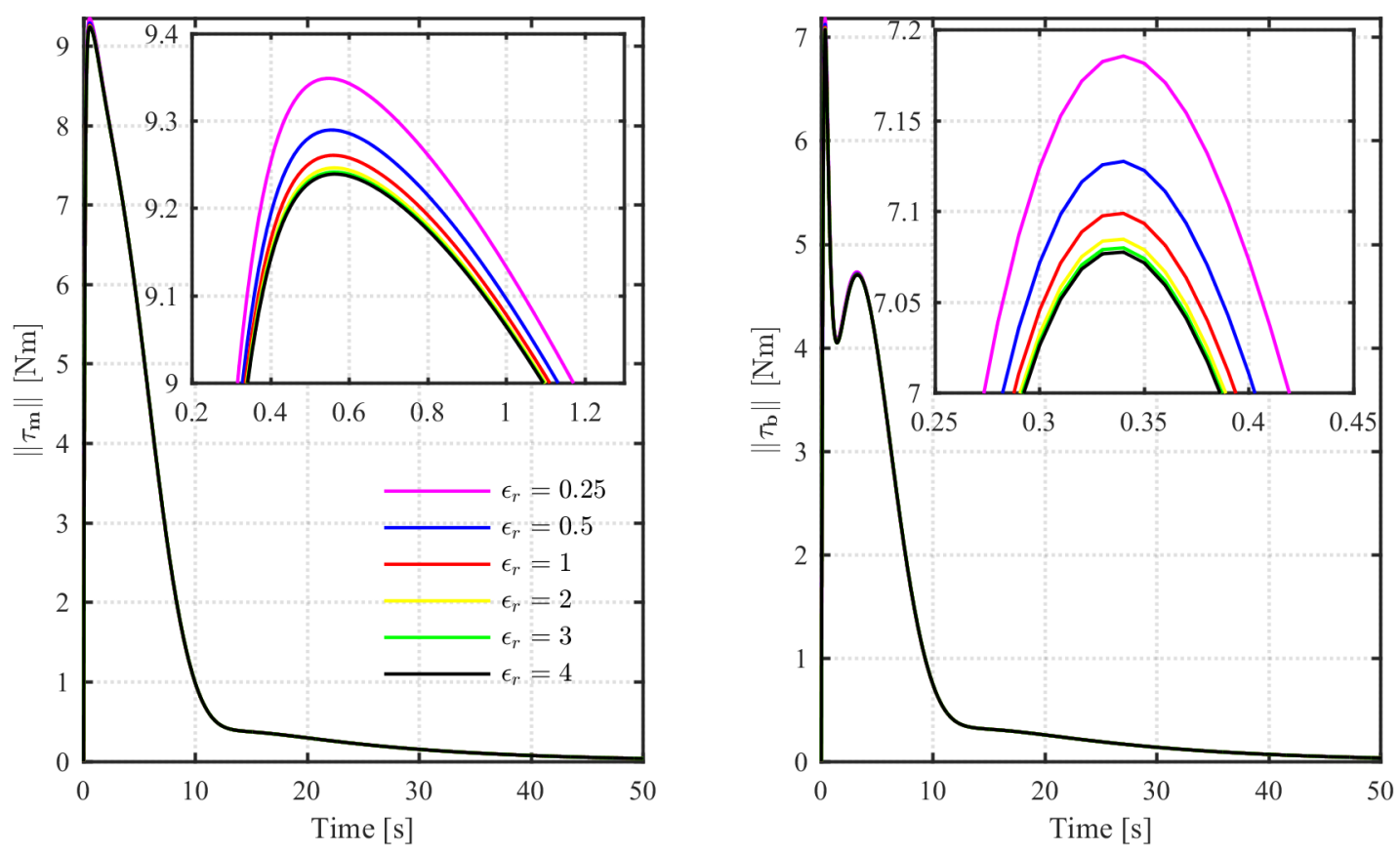

Figure 3.19: Effect of $\varepsilon_{\mathrm{r}}$ in $\Psi$ (3.50a) on the magnitude of the servicer's control inputs. 


\section{Chapter 4}

\section{Detumbling Strategy without Force/Torque Measurements}

\subsection{Detumbling Strategy Overview}

In this Chapter, a detumbling control strategy is presented for the servicer to detumble a noncooperative target with unknown inertial parameters, while being subjected to force/torque limits at the servicer's end-effector. The presented detumbling control strategy is formulated under the assumption that end-effector force/torque measurements are not available. Detumbling of the target is accomplished by designing a robust coordination controller to control the servicer's base satellite and manipulator to track a desired task space detumbling trajectory and to reject the target's gained momentum as its tumbling motion is brought to rest. The proposed robust coordination controller takes into account magnitude constraints on the servicer's control inputs in the controller's design. Furthermore, the desired detumbling trajectory is delineated subjected to force/torque limits at the end-effector and requires only bounds on the target's inertial parameters. This is accomplished by defining the desired velocity and acceleration as a function of the detumbling period using a combination of elementary functions. The detumbling period is then identified from these functions by utilizing bounds on the target's inertial parameters, end-effector force/torque limits and the target's equation of motion. In this Chapter, a numerical simulation study is carried out using a 7-degree-of-freedom manipulator attached to a satellite base to detumble a target using the presented approach and the results 
are reported. Furthermore, an experiment is conducted to evaluate the design procedure used to delineate the desired detumbling trajectory and the results are reported.

\subsubsection{Delineation of End-effector Detumbling Trajectory}

The delineation of the desired detumbling trajectory for the post-grasping phase must be determined while accommodating force/torque limits at the end-effector and without requiring accurate knowledge of the target's inertial parameters. Prior studies in the literature have determined detumbling trajectories that accommodate end-effector force/torque limits constraints with the assumption that the target's inertial parameters are accurately known [6, 7, 9, 65]. This is an unrealistic assumption as the target's inertial parameters cannot be known prior (there is no practical means of measuring remaining propellant in a malfunctioning satellite [8]). Furthermore, estimation techniques in [5, 8, 10, 37, 41, 44] cannot guarantee convergence of estimates of the target's inertial parameters prior to the post-grasping phase. In the face of parameter uncertainty, [66] delineates a detumbling trajectory utilizing conservative estimates of the target's inertial parameters. However, their proposed approach only considers limits on the end-effector's torque and assumes that the location of the target's center of mass is known. Herein, an end-effector detumbling trajectory is derived which accommodates end-effector's force and torque limits and requires only bounds on the target's inertial parameters.

Let $\boldsymbol{v}_{\mathrm{e}}^{\mathrm{d}}\left(\tau_{\mathrm{n}}\right)=\left[\left(\mathbf{v}_{\mathrm{e}}^{\mathrm{d}}\right)^{\mathrm{T}},\left(\mathbf{w}_{\mathrm{e}}^{\mathrm{d}}\right)^{\mathrm{T}}\right]^{\mathrm{T}} \in \mathbb{R}^{6}$ and $\dot{\boldsymbol{v}}_{\mathrm{e}}^{\mathrm{d}}\left(\tau_{\mathrm{n}}\right)=\left[\left(\dot{\mathbf{v}}_{\mathrm{e}}^{\mathrm{d}}\right)^{\mathrm{T}},\left(\dot{\mathbf{w}}_{\mathrm{e}}^{\mathrm{d}}\right)^{\mathrm{T}}\right]^{\mathrm{T}} \in \mathbb{R}^{6}$ denote the endeffector's desired linear and angular velocity and acceleration, respectively, and delineate them using the following functions of normalized time as in [66]:

$$
\begin{gathered}
\left\{\boldsymbol{v}_{\mathrm{e}}^{\mathrm{d}}\left(\tau_{\mathrm{n}}\right)\right\}_{i}=-\mathrm{k}_{i} e^{-\tau_{\mathrm{n}}}+\mathrm{a}_{i} \tau_{\mathrm{n}}^{2}+\mathrm{b}_{i} \tau_{\mathrm{n}}+\mathrm{c}_{i} \\
\left\{\dot{\boldsymbol{v}}_{\mathrm{e}}^{\mathrm{d}}\left(\tau_{\mathrm{n}}\right)\right\}_{i}=\dot{\tau}_{\mathrm{n}}\left(\mathrm{k}_{i} e^{-\tau_{\mathrm{n}}}+2 \mathrm{a}_{i} \tau_{\mathrm{n}}+\mathrm{b}_{i}\right)
\end{gathered}
$$

where $\tau_{\mathrm{n}} \in[0,1]$ is normalized time and $\tau_{\mathrm{n}}=0$ denotes the start of the post-grasping phase. From 4.1 and 4.2, $i=1,2,3, \ldots, 6$ denotes the $i^{t h}$ component of the vector and $\mathrm{k}_{i}, \mathrm{a}_{i}, \mathrm{~b}_{i}$ and $\mathrm{c}_{i}$ are constants to be determined. The time derivative of normalized time, $\dot{\tau}_{\mathrm{n}}$, represents the rate at which detumbling is accomplished and is defined as follows:

$$
\dot{\tau}_{\mathrm{n}}=\frac{1}{T}
$$


where $T$ is the detumbling period (time taken to detumble the target).

In order to determine the desired detumbling trajectory, first the boundary conditions on the end-effector's velocity and acceleration are utilized to determine the coefficients of (4.1) and (4.2). Following this, using the target's equation of motion, 2.42), along with endeffector's force/torque limits, the detumbling period is determined, $T$. With this, the desired end-effector's velocity and acceleration are determined from (4.1) and (4.2). The desired position and orientation of the end-effector can be obtained by integrating the end-effector's desired velocity subjected to the initial position and orientation of the end-effector.

The initial linear and angular velocities of the end-effector in the post-grasping phase are known: $\boldsymbol{v}_{\mathrm{e}}^{\mathrm{d}}\left(\tau_{\mathrm{n}}=0\right)=\boldsymbol{v}_{\mathrm{e}(\text { int })}$, where $\boldsymbol{v}_{\mathrm{e}(\mathrm{int})}=\left[\mathbf{v}_{\mathrm{e}(\text { int })}^{\mathrm{T}}, \mathbf{w}_{\mathrm{e}(\text { int })}^{\mathrm{T}}\right]^{\mathrm{T}}$ denotes the initial linear and angular velocity of the servicer's end-effector. The end-effector's desired final velocity and acceleration are zero: $\left\{\boldsymbol{v}_{\mathrm{e}}^{\mathrm{d}}\left(\tau_{\mathrm{n}}=1\right)\right\}_{i}=0$ and $\left\{\dot{\boldsymbol{v}}_{\mathrm{e}}^{\mathrm{d}}\left(\tau_{\mathrm{n}}=1\right)\right\}_{i}=0$. In addition, it is desirable for the second derivative of the end-effector's velocity to converge to zero at the end of the detumbling period to eliminate any sudden jerks in the system: $\left\{\ddot{\boldsymbol{v}}_{\mathrm{e}}^{\mathrm{d}}\left(\tau_{\mathrm{n}}=1\right)\right\}_{i}=0$. Substituting the above stated initial state conditions into $4.1,4.2$ and $\left\{\ddot{v}_{\mathrm{e}}^{\mathrm{d}}\left(\tau_{\mathrm{n}}\right)\right\}_{i}$ results in the following:

$$
\begin{gathered}
k_{i}=\frac{-1}{1-2.5 e^{-1}}\left\{\boldsymbol{v}_{\mathrm{e}(\mathrm{int})}\right\}_{i} \\
a_{i}=\frac{1}{2} k_{i} e^{-1} \\
b_{i}=-k_{i} e^{-1}-2 a_{i} \\
c_{i}=\left\{\boldsymbol{v}_{\mathrm{e}(\mathrm{int})}\right\}_{i}+k_{i}
\end{gathered}
$$

Substituting the above coefficients, (4.4), into (4.1) and (4.2), and making use of (4.3) results in the following:

$$
\begin{aligned}
\left\{\boldsymbol{v}_{\mathrm{e}}^{\mathrm{d}}\left(\tau_{\mathrm{n}}\right)\right\}_{i} & =-k_{i} e^{-\tau_{\mathrm{n}}}+a_{i} \tau_{\mathrm{n}}^{2}+b_{i} \tau_{\mathrm{n}}+c_{i} \\
& =\left\{\boldsymbol{v}_{\mathrm{e}(\mathrm{int})}\right\}_{i}+\left(-e^{-\tau_{\mathrm{n}}}+\frac{1}{2} e^{-1} \tau_{\mathrm{n}}^{2}-2 e^{-1} \tau_{\mathrm{n}}+1\right) k_{i} \\
& =\left(1+\left(e^{-\tau_{\mathrm{n}}}-\frac{1}{2} e^{-1} \tau_{\mathrm{n}}^{2}+2 e^{-1} \tau_{\mathrm{n}}-1\right) \frac{1}{1-2.5 e^{-1}}\right)\left\{\boldsymbol{v}_{\mathrm{e}(\mathrm{int})}\right\}_{i}
\end{aligned}
$$




$$
\begin{aligned}
\left\{\dot{\boldsymbol{v}}_{\mathrm{e}}^{\mathrm{d}}\left(\tau_{\mathrm{n}}\right)\right\}_{i} & =\dot{\tau}_{\mathrm{n}}\left(k_{i} e^{-\tau_{\mathrm{n}}}+2 a_{i} \tau_{\mathrm{n}}+b_{i}\right) \\
& =\frac{1}{T}\left(e^{-\tau_{\mathrm{n}}}+e^{-1} \tau_{\mathrm{n}}-2 e^{-1}\right) \frac{-1}{1-2.5 e^{-1}}\left\{\boldsymbol{v}_{\mathrm{e}(\mathrm{int})}\right\}_{i}
\end{aligned}
$$

To determine the detumbling period, $T$, the target's equation of motion, $(2.42)$, is segmented into its linear and angular components using the definition of $\Lambda_{\mathbf{t}}, \mathbf{J}_{\mathrm{t}}$ and $\mathbf{c}_{\mathrm{t}}$ in Section 2.6 and the desired velocity and acceleration are substituted as follows:

$$
\begin{gathered}
m_{\mathrm{t}} \dot{\mathbf{v}}_{\mathrm{e}}^{\mathrm{d}}\left(\tau_{\mathrm{n}}\right)+m_{\mathrm{t}} \dot{\mathbf{r}}_{\mathrm{te}}^{\times} \dot{\mathbf{w}}_{\mathrm{e}}^{\mathrm{d}}\left(\tau_{\mathrm{n}}\right)+m_{\mathrm{t}} \dot{\mathbf{r}}_{\mathrm{te}}^{\times} \mathbf{w}_{\mathrm{e}}^{\mathrm{d}}\left(\tau_{\mathrm{n}}\right)=\mathbf{F}_{\mathrm{e}} \\
-m_{\mathrm{t}} \mathbf{r}_{\mathrm{te}}^{\times} \dot{\mathbf{v}}_{\mathrm{e}}^{\mathrm{d}}\left(\tau_{\mathrm{n}}\right)+\left(\mathbf{I}_{\mathrm{t}}-m_{\mathrm{t}} \mathbf{r}_{\mathrm{te}}^{\times} \mathbf{r}_{\mathrm{te}}^{\times}\right) \dot{\mathbf{w}}_{\mathrm{e}}^{\mathrm{d}}\left(\tau_{\mathrm{n}}\right)-m_{\mathrm{t}} \mathbf{r}_{\mathrm{te}}^{\times} \dot{\mathbf{r}}_{\mathrm{te}}^{\times} \mathbf{w}_{\mathrm{e}}^{\mathrm{d}}\left(\tau_{\mathrm{n}}\right)+\mathbf{w}_{\mathrm{e}}^{\mathrm{d}}\left(\tau_{\mathrm{n}}\right) \times \mathbf{I}_{\mathrm{t}} \mathbf{w}_{\mathrm{e}}^{\mathrm{d}}\left(\tau_{\mathrm{n}}\right)=\tau_{\mathrm{e}}
\end{gathered}
$$

From the above equations, the force/torque exerted onto the end-effector are a function of the target's inertial parameters and end-effector's desired acceleration and velocity. The larger the desired acceleration and velocity, the larger the force/torque on the end-effector. In order to respect end-effector's force/torque limits along the delineated detumbling trajectory, it is required to determine the desired velocity and acceleration along which the target's inertial forces do not exceed the servicer's end-effector force/torque limits. This is accomplished by upper bounding the left side of (4.7) and (4.8) and finding the smallest value of $T$ that will ensure that these bounds do not exceed the end-effector's force/torque limits. To proceede, the following lemma is required:

Lemma 1 ( [77]). Let $\boldsymbol{J} \in \mathbb{R}^{3 \times 3}$ be symmetric and positive definite, with minimum and maximum eigenvalues $\lambda_{\min }(\boldsymbol{J})$ and $\lambda_{\max }(\boldsymbol{J})$, respectively. Then, for any $\boldsymbol{x} \in \mathbb{R}^{3}$, the following bound holds:

$$
\left\|\boldsymbol{x}^{\times} \boldsymbol{J x}\right\| \leq\left[\lambda_{\max }^{2}(\boldsymbol{J})-\lambda_{\text {min }}^{2}(\boldsymbol{J})\right]^{1 / 2}\|\boldsymbol{x}\|^{2} .
$$

Upper bounding the left side of (4.7) and 4.8) requires determining an upper bound for the desired velocity and acceleration in (4.5) and (4.6), respectively, for $\tau_{\mathrm{n}} \in[0,1]$. This is accomplished by making use of the following two inequalities for $\tau_{\mathrm{n}} \in[0,1]$ :

$$
1 \geq e^{-\tau_{\mathrm{n}}}-\frac{1}{2} e^{-1} \tau_{\mathrm{n}}^{2}+2 e^{-1} \tau_{\mathrm{n}}
$$




$$
1 \geq e^{-\tau_{\mathrm{n}}}+e^{-1} \tau_{\mathrm{n}}
$$

Let $f_{1}\left(\tau_{\mathrm{n}}\right)=e^{-\tau_{\mathrm{n}}}-\frac{1}{2} e^{-1} \tau_{\mathrm{n}}^{2}+2 e^{-1} \tau_{\mathrm{n}}, f_{2}=e^{-\tau_{\mathrm{n}}}+e^{-1} \tau_{\mathrm{n}}$ and $f_{i}^{\prime}\left(\tau_{\mathrm{n}}\right)=\mathrm{d} f_{i}\left(\tau_{\mathrm{n}}\right) / \mathrm{d} \tau_{\mathrm{n}}$ for $i=$ 1,2. Proof of the inequality in 4.10a can be concluded by considering the following: $f_{1}\left(\tau_{\mathrm{n}}=\right.$ $0,1) \leq 1$ and $f_{1}^{\prime}\left(\tau_{\mathrm{n}}=0,1\right) \leq 0$. Given that $f_{1}^{\prime}\left(\tau_{\mathrm{n}}=0\right)<f_{1}^{\prime}\left(\tau_{\mathrm{n}}=1\right)$ and $f_{1}^{\prime \prime}\left(\tau_{\mathrm{n}}\right)>0$ for $\tau_{\mathrm{n}} \in[0,1)$ (no sign change), it follows that $f_{1}\left(\tau_{\mathrm{n}}\right) \leq 1$ for $\tau_{\mathrm{n}} \in[0,1]$. Similarly, proof of the inequality in $4.10 \mathrm{~b}$ can be concluded by considering the following: $f_{2}\left(\tau_{\mathrm{n}}=0,1\right) \leq 1$. Since $f_{2}^{\prime}\left(\tau_{\mathrm{n}}\right)<0$ for $\tau_{\mathrm{n}} \in[0,1]$, it follows that $f_{2}\left(\tau_{\mathrm{n}}\right) \leq 1$ for $\tau_{\mathrm{n}} \in[0,1]$.

Proof of inequalities (4.10a and (4.10b) allows one to arrive at the required inequalities that will be utilized to upper bound the magnitude of the desired acceleration and velocity, respectively. This is achieved via algebraic manipulation of the individual inequalities in (4.10a) and 4.10b). Using the inequality in 4.10a), a constant value of one is subtracted from both sides of the inequality, then it is multiplied by a positive constant, $\frac{1}{1-2.5 e^{-1}}$, followed by the addition of a constant of one to both sides of the inequality. These steps are demonstrated below:

$$
\begin{aligned}
& 1 \geq f_{1} \\
& 0 \geq f_{1}-1 \\
& 0 \geq\left(f_{1}-1\right) \frac{1}{1-2.5 e^{-1}} \\
& 1 \geq 1+\left(f_{1}-1\right) \frac{1}{1-2.5 e^{-1}}
\end{aligned}
$$

Logically, since the first inequality in (4.11) holds, the last equality in (4.11) also holds. The last equality in 4.11 is utilized to upper bound the end-effector's desired velocity as follows:

$$
\begin{aligned}
\left|\left\{\boldsymbol{v}_{\mathrm{e}}^{\mathrm{d}}\left(\tau_{\mathrm{n}}\right)\right\}_{i}\right| & =\left|\left(1+\left(e^{-\tau_{\mathrm{n}}}-\frac{1}{2} e^{-1} \tau_{\mathrm{n}}^{2}+2 e^{-1} \tau_{\mathrm{n}}-1\right) \frac{1}{1-2.5 e^{-1}}\right)\right|\left|\left\{\boldsymbol{v}_{\mathrm{e}(\mathrm{int})}\right\}_{i}\right| \\
& =\left|1+\left(f_{1}-1\right) \frac{1}{1-2.5 e^{-1}}\right|\left|\left\{\boldsymbol{v}_{\mathrm{e}(\mathrm{int})}\right\}_{i}\right| \\
& \leq\left|\left\{\boldsymbol{v}_{e(\text { int })}\right\}_{i}\right|
\end{aligned} .
$$

From inequality (4.10b), a constant is subtracted from both sides of the inequality as follows:

$$
\begin{aligned}
1 & \geq f_{2} \\
1-2 e^{-1} & \geq f_{2}-2 e^{-1}
\end{aligned}
$$


Making use of the last inequality of (4.13), the desired end-effector acceleration is upper bounded as follows:

$$
\begin{gathered}
\left|\left\{\dot{\boldsymbol{v}}_{\mathrm{e}}^{\mathrm{d}}\left(\tau_{\mathrm{n}}\right)\right\}_{i}\right|=\frac{1}{T}\left|\left(e^{-\tau_{\mathrm{n}}}+e^{-1} \tau_{\mathrm{n}}-2 e^{-1}\right)\right|\left|\frac{-1}{1-2.5 e^{-1}}\right|\left|\left\{\boldsymbol{v}_{e(i n t)}\right\}_{i}\right| \\
=\frac{1}{T}\left|\left(f_{2}-2 e^{-1}\right)\right|\left|\frac{-1}{1-2.5 e^{-1}}\right|\left|\left\{\boldsymbol{v}_{e(i n t)}\right\}_{i}\right| \\
\leq \frac{1}{T} \kappa\left|\left\{\boldsymbol{v}_{e(i n t)}\right\}_{i}\right| \\
\kappa=\left|\left(\frac{1-2 e^{-1}}{1-2.5 e^{-1}}\right)\right|
\end{gathered}
$$

The target's inertial parameters are bounded as follows:

$$
\begin{gathered}
m_{\mathrm{t}} \leq m_{\mathrm{tU}} \\
\lambda_{\min }\left(\mathbf{I}_{\mathrm{t}}\right) \leq\left\|\mathbf{I}_{\mathrm{t}}\right\| \leq \lambda_{\max }\left(\mathbf{I}_{\mathrm{t}}\right) \\
\left|\left\{\mathbf{r}_{\mathrm{te}}\right\}_{x}\right| \leq \mathrm{r}_{\mathrm{teU}} \quad\left|\left\{\mathbf{r}_{\mathrm{te}}\right\}_{y}\right| \leq \mathrm{r}_{\mathrm{teU}} \quad\left|\left\{\mathbf{r}_{\mathrm{te}}\right\}_{z}\right| \leq \mathrm{r}_{\mathrm{teU}}
\end{gathered}
$$

Based on available initial knowledge of the target is it possible to determine an estimate $r_{t e U}$ that satisfies $4.15 \mathrm{c}$ ) such that $\mathrm{r}_{\mathrm{teU}} \leq\left\|\mathbf{r}_{\mathrm{te}}\right\|$.

Making use of (4.12), (4.14) and 4.15), the components of the left side of (4.7) and (4.8) are upper bounded as follows and are required to be upper bounded by the end-effector's force/torque limits:

$$
\begin{aligned}
& \frac{m_{\mathrm{UU}}}{T} \kappa\left(\left|\mathbf{v}_{\mathrm{e}(\mathrm{int}) \mathrm{x}}\right|+\mathrm{r}_{\mathrm{teU}}\left(\left|\mathbf{w}_{\mathrm{e}(\mathrm{int}) \mathrm{z}}\right|+\left|\mathbf{w}_{\mathrm{e}(\text { int }) \mathrm{y}}\right|\right)\right)+m_{\mathrm{tU}} \mathrm{r}_{\mathrm{teU}}\left(\left|\mathbf{w}_{\mathrm{e}(\text { int) } \mathrm{y}}\right|^{2}+\left|\mathbf{w}_{\mathrm{e}(\text { int }) \mathrm{z}}\right|^{2}+\mathrm{w}_{\mathrm{A}}+\mathrm{w}_{\mathrm{B}}\right) \leq \mathrm{F}_{\max } \\
& \frac{m_{\mathrm{UU}}}{T} \kappa\left(\left|\mathbf{v}_{\mathrm{e}(\mathrm{int}) \mathrm{y}}\right|+\mathrm{r}_{\mathrm{teU}}\left(\left|\mathbf{w}_{\mathrm{e}(\mathrm{int}) \mathrm{z}}\right|+\left|\mathbf{w}_{\mathrm{e}(\text { int }) \mathrm{x}}\right|\right)\right)+m_{\mathrm{tU}} \mathrm{r}_{\mathrm{teU}}\left(\left|\mathbf{w}_{\mathrm{e}(\text { int }) \mathrm{x}}\right|^{2}+\left|\mathbf{w}_{\mathrm{e}(\mathrm{int}) \mathrm{z}}\right|^{2}+\mathrm{w}_{\mathrm{A}}+\mathrm{w}_{\mathrm{C}}\right) \leq \mathrm{F}_{\max }
\end{aligned}
$$


$\frac{m_{\mathrm{UU}}}{T} \kappa\left(\left|\mathbf{v}_{\mathrm{e}(\mathrm{int}) \mathrm{z}}\right|+\mathrm{r}_{\mathrm{teU}}\left(\left|\mathbf{w}_{\mathrm{e}(\text { int)y }}\right|+\left|\mathbf{w}_{\mathrm{e}(\mathrm{int}) \mathrm{x}}\right|\right)\right)+m_{\mathrm{tU}} \mathrm{r}_{\mathrm{teU}}\left(\left|\mathbf{w}_{\mathrm{e}(\text { int }) \mathrm{x}}\right|^{2}+\left|\mathbf{w}_{\mathrm{e}(\mathrm{int}) \mathrm{y}}\right|^{2}+\mathrm{w}_{\mathrm{B}}+\mathrm{w}_{\mathrm{C}}\right) \leq \mathrm{F}_{\max }$

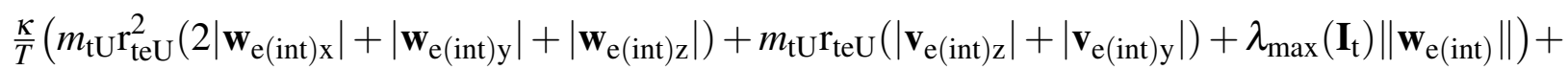

$$
\begin{aligned}
& m_{\mathrm{tU}} \mathrm{r}_{\mathrm{teU}}^{2}\left(\left|\mathbf{w}_{\mathrm{e}(\text { int }) \mathrm{y}}\right|^{2}+\left|\mathbf{w}_{\mathrm{e}(\mathrm{int}) \mathrm{z}}\right|^{2}+\mathrm{w}_{\mathrm{C}}+\mathrm{w}_{\mathrm{B}}+\mathrm{w}_{\mathrm{A}}\right)+G \leq \tau_{\max }
\end{aligned}
$$

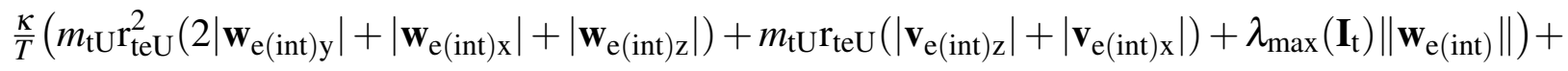

$$
\begin{aligned}
& m_{\mathrm{tU}} \mathrm{r}_{\mathrm{teU}}^{2}\left(\left|\mathbf{w}_{\mathrm{e}(\text { int }) \mathrm{x}}\right|^{2}+\left|\mathbf{w}_{\mathrm{e}(\text { int }) \mathrm{z}}\right|^{2}+\mathrm{w}_{\mathrm{B}}+\mathrm{w}_{\mathrm{C}}+\mathrm{w}_{\mathrm{A}}\right)+G \leq \tau_{\max }
\end{aligned}
$$

$$
\begin{aligned}
& \frac{K}{T}\left(m_{\mathrm{tU}} \mathrm{r}_{\mathrm{teU}}^{2}\left(2\left|\mathbf{w}_{\mathrm{e}(\mathrm{int}) \mathrm{z}}\right|+\left|\mathbf{w}_{\mathrm{e}(\mathrm{int}) \mathrm{x}}\right|+\left|\mathbf{w}_{\mathrm{e}(\mathrm{int}) \mathrm{y}}\right|\right)+m_{\mathrm{tU}} \mathrm{r}_{\mathrm{teU}}\left(\left|\mathbf{v}_{\mathrm{e}(\text { int) }}\right|+\left|\mathbf{v}_{\mathrm{e}(\text { int) }}\right|\right)+\lambda_{\max }\left(\mathbf{I}_{\mathrm{t}}\right)|| \mathbf{w}_{\mathrm{e}(\text { int })} \|\right)+ \\
& m_{\mathrm{tU}} \mathrm{r}_{\mathrm{teU}}^{2}\left(\left|\mathbf{w}_{\mathrm{e}(\text { int }) \mathrm{x}}\right|^{2}+\left|\mathbf{w}_{\mathrm{e}(\text { int) }}\right|^{2}+\mathrm{w}_{\mathrm{A}}+\mathrm{w}_{\mathrm{C}}+\mathrm{w}_{\mathrm{B}}\right)+G \leq \tau_{\max }
\end{aligned}
$$

where $\quad \mathrm{w}_{\mathrm{A}}=\left|\mathbf{w}_{\mathrm{e}(\text { int) } \mathrm{x}}\right|\left|\mathbf{w}_{\mathrm{e}(\text { int)y }}\right|, \quad \mathrm{w}_{\mathrm{B}}=\left|\mathbf{w}_{\mathrm{e}(\text { int) }}\right|\left|\mathbf{w}_{\mathrm{e}(\text { int)z } \mathrm{z}}\right|, \quad \mathrm{w}_{\mathrm{C}}=\left|\mathbf{w}_{\mathrm{e}(\text { int)y }}\right|\left|\mathbf{w}_{\mathrm{e}(\text { int)z } \mathrm{z}}\right|, \quad G=$ $\left(\lambda_{\max }^{2}\left(\mathbf{I}_{\mathrm{t}}\right)-\lambda_{\min }^{2}\left(\mathbf{I}_{\mathrm{t}}\right)\right)^{\frac{1}{2}}\left\|\mathbf{w}_{\mathrm{e}(\mathrm{int})}\right\|^{2}$ and Lemma 1 in [77] was utilized to upper bound $\| \mathbf{w}_{\mathrm{e}}^{\mathrm{d}}\left(\tau_{\mathrm{n}}\right) \times$ $\mathbf{I}_{\mathrm{t}} \mathbf{w}_{\mathrm{e}}^{\mathrm{d}}\left(\tau_{\mathrm{n}}\right)\left\|\leq\left(\lambda_{\max }^{2}\left(\mathbf{I}_{\mathrm{t}}\right)-\lambda_{\min }^{2}\left(\mathbf{I}_{\mathrm{t}}\right)\right)^{\frac{1}{2}}\right\| \mathbf{w}_{\mathrm{e}(\text { int })} \|^{2}$ in $G$.

From (4.16a) through (4.16f), the detumbling period can be solved from each equation and will be denoted as $T_{i}$ for $i=1,2,3, \ldots, 6$ corresponding to each equation in 4.16). From this, the detumbling period is determined as follows:

$$
T \geq \max \left\{T_{i}: i=1,2,3, \ldots, 6\right\}
$$

Utilizing $T$, the desired end-effector velocity and acceleration are delineated by (4.5) and (4.6). The desired position of the end-effector can be obtain by integrating the desired linear velocity. The end-effector's desired orientation can be obtained by integrating the time derivative of the quaternion representing the end-effector's attitude with the use of the desired angular velocity. 
The desired end-effector position is denoted as $\mathbf{x}_{\mathrm{e}}^{\mathrm{d}}$ and the desired end-effector orientation is denoted as the following unit quaternion $\mathbf{q}_{\mathrm{e}}^{\mathrm{d}}$.

Solving for the detumbling period from (4.16) provides additional insight into the design requirements of the servicer satellite. Under the requirement that $T>0$, it is required that servicer be designed so that $\mathrm{F}_{\max }$ and $\tau_{\max }$ are large enough to satisfy the following:

$$
\begin{gathered}
\mathrm{F}_{\mathrm{max}}-m_{\mathrm{tU}} \mathrm{r}_{\mathrm{teU}}\left(\left|\mathbf{w}_{\mathrm{e}(\text { int }) \mathrm{y}}\right|^{2}+\left|\mathbf{w}_{\mathrm{e}(\text { int }) \mathrm{z}}\right|^{2}+\mathrm{w}_{\mathrm{A}}+\mathrm{w}_{\mathrm{B}}\right)>0 \\
\mathrm{~F}_{\mathrm{max}}-m_{\mathrm{tU}} \mathrm{r}_{\mathrm{teU}}\left(\left|\mathbf{w}_{\mathrm{e}(\text { int }) \mathrm{x}}\right|^{2}+\left|\mathbf{w}_{\mathrm{e}(\text { int }) \mathrm{z}}\right|^{2}+\mathrm{w}_{\mathrm{A}}+\mathrm{w}_{\mathrm{C}}\right)>0 \\
\mathrm{~F}_{\mathrm{max}}-m_{\mathrm{tU}} \mathrm{r}_{\mathrm{teU}}\left(\left|\mathbf{w}_{\mathrm{e}(\text { int }) \mathrm{x}}\right|^{2}+\left|\mathbf{w}_{\mathrm{e}(\text { int }) \mathrm{y}}\right|^{2}+\mathrm{w}_{\mathrm{B}}+\mathrm{w}_{\mathrm{C}}\right)>0 \\
\tau_{\mathrm{max}}-m_{\mathrm{tU}} \mathrm{r}_{\mathrm{teU}}^{2}\left(\left|\mathbf{w}_{\mathrm{e}(\text { int }) \mathrm{y}}\right|^{2}+\left|\mathbf{w}_{\mathrm{e}(\text { int }) \mathrm{z}}\right|^{2}+\mathrm{w}_{\mathrm{C}}+\mathrm{w}_{\mathrm{B}}+\mathrm{w}_{\mathrm{A}}\right)-G>0 \\
\tau_{\mathrm{max}}-m_{\mathrm{tU}} \mathrm{r}_{\mathrm{teU}}^{2}\left(\left|\mathbf{w}_{\mathrm{e}(\text { int }) \mathrm{x}}\right|^{2}+\left|\mathbf{w}_{\mathrm{e}(\text { int }) \mathrm{z}}\right|^{2}+\mathrm{w}_{\mathrm{B}}+\mathrm{w}_{\mathrm{C}}+\mathrm{w}_{\mathrm{A}}\right)-G>0 \\
\tau_{\max }-m_{\mathrm{tU}} \mathrm{r}_{\mathrm{teU}}^{2}\left(\left|\mathbf{w}_{\mathrm{e}(\text { int }) \mathrm{x}}\right|^{2}+\left|\mathbf{w}_{\mathrm{e}(\text { int }) \mathrm{y}}\right|^{2}+\mathrm{w}_{\mathrm{A}}+\mathrm{w}_{\mathrm{C}}+\mathrm{w}_{\mathrm{B}}\right)-G>0
\end{gathered}
$$

\subsubsection{Desired Base Attitude}

In the post-grasping phase, as the target's tumbling motion is brought to rest its momentum is transferred to the servicer. The gained momentum must be rejected in order to avoid tumbling of the combined servicer/target system. This is accomplished by simply setting the servicer's base desired angular velocity as $\mathbf{w}_{\mathrm{b}}^{\mathrm{d}}=0$. An additional task such as re-orienting the servicer's base to a desired attitude during the detumbling procedure can still be accomplished and will still achieve rejection of the target's gained momentum. In this case the servicer's base desired attitude will be denoted as the following unit quaternion $\mathbf{q}_{b}^{\mathrm{d}}$. 


\subsubsection{Robust Trajectory Tracking Controller}

This subsection presents the development of a robust coordination controller to control the servicer's manipulator and satellite base to track the desired detumbling trajectory at the endeffector and to accommodate the target's gained momentum as its tumbling motion is brought to rest. The development of the robust coordination controller does not require force/torque measurements at the end-effector and takes into account magnitude constraints on the servicer's manipulator and satellite base attitude control inputs in the design of the controller.

It is assumed that the presented desired detumbling trajectory in Section 4.1.1 lies within the capability of the servicer's actuators. This is a reasonable assumption because it implies there exists a control input that satisfies the magnitude constraints on the servicer's control input such that detumbling of the target can be achieved. This can be be observed by upper bounding the servicer's control inputs, $\tau$, from 2.37) while making use of the desired detumbling velocity and acceleration as follows:

$$
\|\tau\| \leq\|\mathbf{M A}\|\left\|\dot{\xi}^{\mathrm{d}}\right\|+\left\|\left(\mathbf{M} \overline{\mathbf{c}}_{\mathrm{A}}+\mathbf{c}\right)\right\|+\left\|\left(\mathbf{J}-\mathbf{M} \mathbf{J}_{\mathrm{A}}\right)\right\| \mathrm{K}_{\max }
$$

where $\mathrm{K}_{\max }=\max \left\{\mathrm{F}_{\max }, \tau_{\max }\right\}$. Upper bounding $\left\|\left(\mathbf{M} \overline{\mathbf{c}}_{\mathrm{A}}+\mathbf{c}\right)\right\|$ along the desired trajectory is difficult as it not only depends on $\xi^{\mathrm{d}}$ but also on the servicer's manipulator joint velocities and base linear velocity. However, it can be neglected as the motion of the servicer's manipulator and base are slow. Similarly, the magnitude of $\|\mathbf{M A}\|\left\|\dot{\xi}^{\mathrm{d}}\right\|$ can be neglected due to the low desired acceleration and the fact that $\dot{\mathbf{w}}_{\mathrm{b}}^{\mathrm{d}}=0$. As a result, the upper bound on the servicer's manipulator and attitude control inputs are approximated as follows:

$$
\begin{gathered}
\left\|\tau_{\mathrm{m}}\right\| \leq\left\|\mathbf{J}_{\mathrm{e}}\right\| \mathrm{K}_{\max } \\
\left\|\tau_{\mathrm{b}}\right\| \leq\left\|\mathbf{J}_{\mathrm{R}}\right\| \mathrm{K}_{\max } \\
\left(\mathbf{J}-\mathbf{M J}_{\mathrm{A}}\right)=\left[\begin{array}{l}
\mathbf{J}_{\mathrm{e}} \\
\mathbf{J}_{\mathrm{R}}
\end{array}\right]
\end{gathered}
$$

where $\mathbf{J}_{\mathrm{e}} \in \mathbb{R}^{m \times 6}$ and $\mathbf{J}_{\mathrm{R}} \in \mathbb{R}^{3 \times 6}$.

From (4.20a) and 4.20b), the upper bound on manipulator and attitude control inputs are a 
function of the maximum force/torque that may occur when detumbling the target. However, these bounds only hold provided that servicer's end-effector's position/orientation and velocity tracking errors are zero and servicer's base attitude and angular velocity tracking errors are zero. Non-zero tracking errors may require the servicer's control input to exceed these bounds. In practice, these bounds will be fixed and cannot be exceeded due to actuator limitation and may lead to instability if not taken into account in the controller's design. The remainder of this subsection takes into account magnitude limits on the servicer's control inputs in the controller's design to track the desired detumbling trajectory and reject the gained target's momentum.

To achieve this the position and orientation error of the end-effector and base attitude are defined as follows:

$$
\begin{gathered}
\mathbf{e}_{\mathrm{P}}=\left[\begin{array}{c}
\mathbf{x}_{\mathrm{e}}-\mathbf{x}_{\mathrm{e}}^{\mathrm{d}} \\
\left(\delta \mathbf{q}_{\mathrm{v}}\right)_{\mathrm{e}} \\
\left(\delta \mathbf{q}_{\mathrm{v}}\right)_{\mathrm{b}}
\end{array}\right]=\left[\begin{array}{c}
\mathbf{e}_{\mathrm{pe}} \\
\left(\delta \mathbf{q}_{\mathrm{v}}\right)_{\mathrm{e}} \\
\left(\delta \mathbf{q}_{\mathrm{v}}\right)_{\mathrm{b}}
\end{array}\right] \\
\mathbf{e}_{\mathrm{V}}=\left[\begin{array}{c}
\mathbf{v}_{\mathrm{e}}-\mathbf{v}_{\mathrm{e}}^{\mathrm{d}} \\
\mathbf{w}_{\mathrm{e}}-\mathbf{w}_{\mathrm{e}}^{\mathrm{d}} \\
\mathbf{w}_{\mathrm{b}}-\mathbf{w}_{\mathrm{b}}^{\mathrm{d}}
\end{array}\right]=\left[\begin{array}{c}
\mathbf{e}_{\mathrm{ve}} \\
\mathbf{e}_{\mathrm{we}} \\
\mathbf{e}_{\mathrm{wb}}
\end{array}\right]
\end{gathered}
$$

where $\left(\delta \mathbf{q}_{\mathbf{v}}\right)_{(\cdot)}$ denote the vector component of the quaternion associated with the attitude tracking error of the end-effector, $(\cdot)=\mathrm{e}$, and base, $(\cdot)=\mathrm{b} . \quad \delta \mathbf{q}_{\mathrm{v}}=\mathrm{q}_{\mathrm{s}} \mathbf{q}_{\mathrm{v}}^{\mathrm{d}}+\mathbf{q}_{\mathrm{v}} \times \mathbf{q}_{\mathrm{v}}^{\mathrm{d}}-\mathbf{q}_{\mathrm{v}} \mathrm{q}_{\mathrm{s}}^{\mathrm{d}} \in$ $\mathbb{R}^{3}$ where the scalar and vector components of the quaternion are denoted by $(\cdot)_{\mathrm{s}}$ and $(\cdot)_{\mathrm{v}}$, respectively, and $(\cdot)^{\mathrm{d}}$ represents the desired quaternion value. Making use of (4.21), a sliding variable, $\mathbf{s}_{1} \in \mathbb{R}^{9}$, is defined as:

$$
\mathbf{s}_{1}=\mathbf{e}_{\mathrm{V}}+\mathbf{K}_{\mathrm{P}} \mathbf{e}_{\mathrm{P}}
$$

where $\mathbf{K}_{\mathrm{P}}=\operatorname{diag}\left[\mathbf{K}_{\mathrm{PEL}}, \mathrm{K}_{\mathrm{PEA}} \mathbf{E}_{3}, \mathrm{~K}_{\mathrm{PBA}} \mathbf{E}_{3}\right]$ and $0<\mathbf{K}_{\mathrm{PEL}} \in \mathbb{R}^{3 \times 3}, \mathrm{~K}_{\mathrm{PEA}} \in \mathbb{R}$ and $\mathrm{K}_{\mathrm{PBA}} \in \mathbb{R}$ are positive gains. Let $\mathbf{s}_{1}=\left[\mathbf{s}_{1 \mathrm{pe}}^{\mathrm{T}}, \mathbf{s}_{1 \mathrm{we}}^{\mathrm{T}}, \mathbf{s}_{1 \mathrm{wb}}^{\mathrm{T}}\right]^{\mathrm{T}}$ where $\mathbf{s}_{1 \mathrm{pe}} \in \mathbb{R}^{3}, \mathbf{s}_{1 \mathrm{we}} \in \mathbb{R}^{3}$ and $\mathbf{s}_{1 \mathrm{wb}} \in \mathbb{R}^{3}$, it follows from (4.21) and (4.22) that:

$$
\begin{gathered}
\mathbf{s}_{1 \mathrm{pe}}=\mathbf{e}_{\mathrm{ve}}+\mathbf{K}_{\mathrm{PEL}} \mathbf{e}_{\mathrm{pe}} \\
\mathbf{s}_{1 \mathrm{we}}=\mathbf{e}_{\mathrm{we}}+\mathrm{K}_{\mathrm{PEA}}\left(\delta \mathbf{q}_{\mathrm{v}}\right)_{\mathrm{e}} \\
\mathbf{s}_{1 \mathrm{wb}}=\mathbf{e}_{\mathrm{wb}}+\mathrm{K}_{\mathrm{PBA}}\left(\delta \mathbf{q}_{\mathrm{v}}\right)_{\mathrm{b}}
\end{gathered}
$$


Boundedness of $\mathbf{s}_{1}$ will result in $\mathbf{e}_{\mathrm{V}}$ and $\mathbf{e}_{\mathrm{P}}$ also being ultimately bounded. For the system in 4.23a), this follows since it represents an exponentially stable system that is disturbed by an ultimately bounded input in $\mathbf{s}_{1 \mathrm{pe}}$. Similarly, boundedness of $\mathbf{e}_{\mathrm{w}(\cdot)}$ and $\left(\delta \mathbf{q}_{\mathrm{v}}\right)_{(\cdot)}$ for a bounded $\mathbf{s}_{1 \mathrm{w}(\cdot)}$ in the systems described in $4.23 \mathrm{~b},(\cdot)=\mathrm{e}$, and $\left.4.23 \mathrm{c}\right),(\cdot)=\mathrm{b}$, follows from Lemma 1 in [16].

The controller design takes into account magnitude constraints on the servicer's control inputs by modelling the servicer's control inputs, $\tau$ in $(2.43)$, by a smooth hyperbolic tangent function [77]:

$$
\begin{gathered}
\tau=f(\mathbf{V}) \\
\{f(\mathbf{V})\}_{i}=\left\{\mathbf{U}_{\mathbf{M}}\right\}_{i} \tanh \left(\frac{\{\mathbf{V}\}_{i}}{\left\{\mathbf{U}_{\mathbf{M}}\right\}_{i}}\right) \\
\dot{\mathbf{V}}=\bar{f}^{-1} \mathbf{U}
\end{gathered}
$$

where $i=1,2,3, \ldots,(m+3)$ and the $i^{\text {th }}$ element of $\mathbf{U}_{\mathrm{M}} \in \mathbb{R}^{(m+3)}$ denotes the control input magnitude limit of the $i^{\text {th }}$ servicer's actuator. $\bar{f}=\partial f / \partial \mathbf{V}, \mathbf{V} \in \mathbb{R}^{m+3}$ and $\mathbf{U} \in \mathbb{R}^{m+3}$. From the above model of the servicer's control input, it can be concluded that servicer's control input will always satisfies its magnitude constraint: $\{\tau\}_{i} \leq\left\{\mathbf{U}_{\mathbf{M}}\right\}_{i}$ for $i=1,2,3, \ldots,(m+3)$. All functions of the resultant system comprising of (2.43) and (4.24) are smooth, and hence, the use of the backstepping technique is feasible to design the auxiliary signal $\mathbf{U}$.

The use of the hyperbolic tangent function to model the servicer's control inputs is an alternative to the use of a discontinuous auxiliary system designed to account for control input magnitude limits as in [30]. The latter adds additional complexity in the tuning of the auxiliary system and analysis of the resultant controller, while the former results in a simpler control design and analysis of the resultant controller with the use of the backstepping technique.

The design of $\mathbf{U}$ is described in the following steps:

Step 1: Design of the virtual controller for $f(\mathbf{V})$. The dynamic equation for $\mathbf{s}_{1}$ is obtain using (4.22) and (2.43):

$$
\overline{\mathbf{M}}_{\mathrm{o}} \dot{\mathbf{s}}_{1}=\mathbf{A}^{\mathrm{T}} f(\mathbf{V})-\overline{\mathbf{c}}_{\mathrm{o}}-\Delta \overline{\mathbf{c}}-\overline{\mathbf{M}}_{\mathrm{o}} \dot{\xi}^{\mathrm{d}}-\Delta \overline{\mathbf{M}} \dot{\xi}+\overline{\mathbf{M}}_{\mathrm{o}} \mathbf{K}_{\mathrm{P}} \dot{\mathbf{e}}_{\mathrm{p}}
$$

where $\overline{\mathbf{M}}_{\mathrm{o}}=\mathbf{M}_{\mathrm{s}}$ and $\overline{\mathbf{c}}_{\mathrm{o}}=\mathbf{c}_{\mathrm{s}}$ denote the nominal component of the servicer/target mass matrix and nominal component of the servicer's nonlinear velocity dependent terms, respectively. 
$\Delta \overline{\mathbf{M}}=\left[\begin{array}{ll}\mathbf{J}_{\mathrm{s}} \Lambda_{\mathrm{t}} & \mathbf{0}\end{array}\right] \in \mathbb{R}^{(6+3) \times(6+3)}$ and $\Delta \overline{\mathbf{c}}=\mathbf{J}_{\mathrm{s}}\left(\mathbf{J}_{\mathrm{t}}^{-\mathrm{T}} \mathbf{c}_{\mathrm{t}}-\Lambda_{\mathrm{t}} \mathbf{J}_{\mathrm{t}} \mathbf{J}_{\mathrm{t}}^{-1} \boldsymbol{v}_{\mathrm{e}}\right)$ so that $\overline{\mathbf{M}}=\overline{\mathbf{M}}_{\mathrm{o}}+\Delta \overline{\mathbf{M}}$ and $\overline{\mathbf{c}}=\overline{\mathbf{c}}_{\mathrm{o}}+\Delta \overline{\mathbf{c}}$.

Utilizing the definition of $\Lambda_{\mathrm{t}}, \mathbf{J}_{\mathrm{t}}$ and $\mathbf{c}_{\mathrm{t}}$ in Section 2.6, the evaluation of $\Delta \overline{\mathbf{c}}$ is as follows:

$$
\begin{aligned}
\Delta \overline{\mathbf{c}} & =\mathbf{J}_{\mathrm{s}}\left(\mathbf{J}_{\mathrm{t}}^{-\mathrm{T}} \mathbf{c}_{\mathrm{t}}-\Lambda_{\mathrm{t}} \dot{\mathbf{J}}_{\mathrm{t}} \mathbf{J}_{\mathrm{t}}^{-1} \mathbf{v}_{\mathrm{e}}\right) \\
& =\mathbf{J}_{\mathrm{s}}\left[\begin{array}{c}
m_{\mathrm{t}} \dot{\mathbf{r}}_{\mathrm{te}}^{\times} \mathbf{w}_{\mathrm{t}} \\
\mathbf{w}_{\mathrm{t}} \times \mathbf{I}_{\mathrm{t}} \mathbf{w}_{\mathrm{t}}-m_{\mathrm{t}} \mathbf{r}_{\mathrm{te}}^{\times} \dot{\mathbf{r}}_{\mathrm{te}}^{\times} \mathbf{w}_{\mathrm{t}}
\end{array}\right] \\
& =\mathbf{J}_{\mathrm{s}}\left(\gamma_{1}+\gamma_{2}\right)
\end{aligned}
$$

where with the use of $\mathbf{w}_{\mathrm{t}}=\mathbf{w}_{\mathrm{e}}=\mathbf{e}_{\mathrm{we}}+\mathbf{w}_{\mathrm{e}}^{\mathrm{d}}$ in $4.26, \gamma_{1}$ and $\gamma_{2}$ are defined as follows:

$$
\begin{gathered}
\gamma_{1}=\left[\begin{array}{c}
m_{\mathrm{t}} \mathbf{w}_{\mathrm{t}}^{\mathrm{d} \times} \mathbf{r}_{\mathrm{te}}^{\times} \mathbf{w}_{\mathrm{t}}^{\mathrm{d}} \\
\mathbf{w}_{\mathrm{t}}^{\mathrm{d}} \times \mathbf{I}_{\mathrm{t}} \mathbf{w}_{\mathrm{t}}^{\mathrm{d}}-m_{\mathrm{t}} \mathbf{r}_{\mathrm{te}}^{\times} \mathbf{w}_{\mathrm{t}}^{\mathrm{d} \times} \mathbf{r}_{\mathrm{te}}^{\times} \mathbf{w}_{\mathrm{t}}^{\mathrm{d}}
\end{array}\right] \\
\gamma_{2}=\left[\begin{array}{c}
m_{\mathrm{t}} \gamma_{2 \mathrm{a}} \\
\gamma_{2 \mathrm{~b}}+m_{\mathrm{t}} \mathbf{r}_{\mathrm{te}}^{\times} \gamma_{2 \mathrm{a}}
\end{array}\right]
\end{gathered}
$$

where $\gamma_{2 \mathrm{a}}=\mathbf{e}_{\mathrm{we}}^{\times} \mathbf{r}_{\mathrm{te}}^{\times} \mathbf{e}_{\mathrm{we}}+2 \mathbf{e}_{\mathrm{we}}^{\times} \mathbf{r}_{\mathrm{te}}^{\times} \mathbf{w}_{\mathrm{e}}^{\mathrm{d}}$ and $\gamma_{2 \mathrm{~b}}=\mathbf{e}_{\mathrm{we}} \times \mathbf{I}_{\mathrm{t}} \mathbf{e}_{\mathrm{we}}+\mathbf{e}_{\mathrm{we}} \times \mathbf{I}_{\mathrm{t}} \mathbf{w}_{\mathrm{e}}^{\mathrm{d}}+\mathbf{w}_{\mathrm{e}}^{\mathrm{d}} \times \mathbf{I}_{\mathrm{t}} \mathbf{e}_{\mathrm{we}}$.

Making use of (4.15) and Lemma 1 in [77], the components of (4.27a are upper bounded as:

$$
\begin{gathered}
\left\|m_{\mathrm{t}} \mathbf{w}_{\mathrm{t}}^{\mathrm{d} \times} \mathbf{r}_{\mathrm{te}}^{\times} \mathbf{w}_{\mathrm{t}}^{\mathrm{d}}\right\| \leq m_{\mathrm{tU}}\left\|\mathbf{r}_{\mathrm{te}}\right\|\left\|\mathbf{w}_{\mathrm{e}}^{\mathrm{d}}\right\|^{2} \\
\left\|\mathbf{w}_{\mathrm{t}}^{\mathrm{d}} \times \mathbf{I}_{\mathrm{t}} \mathbf{w}_{\mathrm{t}}^{\mathrm{d}}\right\| \leq\left(\lambda_{\text {max }}^{2}\left(\mathbf{I}_{\mathrm{t}}\right)-\lambda_{\min }^{2}\left(\mathbf{I}_{\mathrm{t}}\right)\right)^{\frac{1}{2}}\left\|\mathbf{w}_{\mathrm{e}}^{\mathrm{d}}\right\|^{2} \\
\left\|m_{\mathrm{t}} \mathbf{r}_{\mathrm{te}}^{\times} \mathbf{w}_{\mathrm{t}}^{\mathrm{d} \times} \mathbf{r}_{\mathrm{te}}^{\times} \mathbf{w}_{\mathrm{t}}^{\mathrm{d}}\right\| \leq m_{\mathrm{tU}}\left\|\mathbf{r}_{\mathrm{te}}\right\|^{2}\left\|\mathbf{w}_{\mathrm{e}}^{\mathrm{d}}\right\|^{2}
\end{gathered}
$$

Utilizing (4.28) the magnitude of $\gamma_{1}$ is upper bounded as:

$$
\begin{gathered}
\left\|\gamma_{1}\right\| \leq h\left\|\mathbf{w}_{\mathrm{e}}^{\mathrm{d}}\right\|^{2} \\
h=\left[\left(m_{\mathrm{tU}}\left\|\mathbf{r}_{\mathrm{te}}\right\|\right)^{2}+\left(\left[\lambda_{\text {max }}^{2}\left(\mathbf{I}_{\mathrm{t}}\right)-\lambda_{\text {min }}^{2}\left(\mathbf{I}_{\mathrm{t}}\right)\right]^{\frac{1}{2}}+m_{t}\left\|\mathbf{r}_{\mathrm{te}}\right\|^{2}\right)^{2}\right]^{\frac{1}{2}} .
\end{gathered}
$$

As a result of the above, $\Delta \overline{\mathbf{c}}=\mathbf{J}_{\mathrm{s}} \gamma_{1}+\mathbf{J}_{\mathrm{s}} \gamma_{2}$, and with the use of 4.29a), $\mathbf{J}_{\mathrm{s}} \gamma_{1}$ can be upper 
bounded as follows:

$$
\left\|\mathbf{J}_{\mathrm{s}} \gamma_{1}\right\| \leq h\left\|\mathbf{J}_{\mathrm{s}}\right\|\left\|\mathbf{w}_{\mathrm{e}}^{\mathrm{d}}\right\|^{2} \leq \theta
$$

where $\theta$ is known positive constant since $h$ can be computed utilizing bounds on the target's inertial parameters, $\mathbf{J}_{\mathrm{s}}$ is a function of known servicer's inertial and geometric parameters, and $\mathbf{w}_{\mathrm{e}}^{\mathrm{d}}$ is shown to be bounded in Section 4.1.1.

To design the virtual controller for $f(\mathbf{V})$, we utilize the following Lyapunov function candidate from [77] which is radially unbounded and globally positive-definite function:

$$
\mathrm{V}_{\mathrm{P} 1}=\sum_{i=1}^{9} \ln \left(\cosh \left(\left\{\mathbf{s}_{1}\right\}_{i}\right)\right)
$$

Taking the time derivative of (4.31) and making use of (4.25) and (4.26), results in the following expression for $\dot{\mathrm{V}}_{\mathrm{P} 1}$ :

$$
\begin{aligned}
\dot{\mathrm{V}}_{\mathrm{P} 1} & =\tanh ^{\mathrm{T}}\left(\mathbf{s}_{1}\right) \dot{\mathbf{s}}_{1} \\
& =\tanh ^{\mathrm{T}}\left(\mathbf{s}_{1}\right) \overline{\mathbf{M}}_{\mathrm{o}}^{-1}\left(\mathbf{A}^{\mathrm{T}} f(\mathbf{V})-\overline{\mathbf{c}}_{\mathrm{o}}-\mathbf{J}_{\mathrm{s}}\left(\gamma_{1}+\gamma_{2}\right)-\overline{\mathbf{M}}_{0} \dot{\xi}^{\mathrm{d}}-\Delta \overline{\mathbf{M}} \dot{\xi}+\overline{\mathbf{M}}_{\mathrm{o}} \mathbf{K}_{\mathrm{P}} \dot{\mathbf{e}}_{\mathrm{p}}\right)
\end{aligned}
$$

where $\tanh (\mathbf{x})=\left[\tanh \left(x_{1}\right), \tanh \left(x_{2}\right), \ldots, \tanh \left(x_{n}\right)\right]^{\mathrm{T}}$ for any vector $\mathbf{x} \in \mathbb{R}^{n}$

Considering $f(\mathbf{V})$ as a virtual controller, the following virtual controller is proposed:

$$
f(\mathbf{V})^{\mathrm{d}}=\mathbf{A}^{-\mathrm{T}}\left(\overline{\mathbf{c}}_{\mathrm{o}}+\overline{\mathbf{M}}_{\mathrm{o}} \dot{\xi}^{\mathrm{d}}-\overline{\mathbf{M}}_{\mathrm{o}} \mathbf{K}_{\mathrm{P}} \dot{\mathbf{e}}_{\mathrm{P}}-\overline{\mathbf{M}}_{\mathrm{o}} \mathrm{K}_{1} \tanh \left(\mathbf{s}_{1}\right)-\psi_{\mathrm{P} 1}\right)
$$

with $\mathrm{K}_{1}$ as a positive constant and $\psi_{\mathrm{P} 1} \in \mathbb{R}^{9}$ is a robust compensator defined as in [28]:

$$
\psi_{\mathrm{P} 1 i}=\theta \tanh \left(\frac{9 \mathrm{k}_{u} \theta\left\{\overline{\mathbf{M}}_{\mathrm{o}}^{-1} \tanh \left(\mathbf{s}_{1}\right)\right\}_{i}}{\varepsilon_{1}}\right) \quad \text { for } i=1,2,3, \ldots, 9
$$

where $\mathrm{k}_{u}=0.2785$ [52] and $\varepsilon_{1}$ is a small positive scalar. The hyperbolic tangent function has the following property [52]:

$$
0 \leq|x|-x \tanh \left(\frac{x}{\varepsilon_{u}}\right) \leq \mathrm{k}_{\mathrm{u}} \varepsilon_{u}
$$

for any $\varepsilon_{\mathrm{u}}>0$ and any $x \in \mathbb{R}$. It was also utilized to prevent chattering of $f(\mathbf{V})$ [57]. Making 
use of this property, the following bound is obtained:

$$
\begin{aligned}
\left\|\tanh ^{\mathrm{T}}\left(\mathbf{s}_{1}\right) \overline{\mathbf{M}}_{\mathrm{o}}^{-1}\right\| \theta-\tanh ^{\mathrm{T}}\left(\mathbf{s}_{1}\right) \overline{\mathbf{M}}_{\mathrm{o}}^{-1} \psi_{\mathrm{P} 1} & \leq \sum_{1}^{9}\left(\left|\left\{\tanh ^{\mathrm{T}}\left(\mathbf{s}_{1}\right) \overline{\mathbf{M}}_{\mathrm{o}}^{-1}\right\}_{i}\right| \theta-\left\{\tanh ^{\mathrm{T}}\left(\mathbf{s}_{1}\right) \overline{\mathbf{M}}_{\mathrm{o}}^{-1}\right\}_{i}\left\{\psi_{\mathrm{P} 1}\right\}_{i}\right) \\
& \leq \varepsilon_{1}
\end{aligned}
$$

Utilizing (4.33) and (4.36) in (4.32), results in:

$$
\begin{aligned}
\dot{\mathrm{V}}_{\mathrm{P} 1} & =\tanh ^{\mathrm{T}}\left(\mathbf{s}_{1}\right) \overline{\mathbf{M}}_{\mathrm{o}}^{-1}\left(\mathbf{A}^{\mathrm{T}} \mathbf{s}_{2}+\mathbf{A}^{\mathrm{T}} f(\mathbf{V})^{\mathrm{d}}-\overline{\mathbf{c}}_{\mathrm{o}}-\mathbf{J}_{\mathrm{s}}\left(\gamma_{1}+\gamma_{2}\right)-\overline{\mathbf{M}}_{\mathrm{o}} \dot{\xi}+\overline{\mathbf{M}}_{\mathrm{o}} \mathbf{K}_{\mathrm{P}} \dot{\mathbf{e}}_{\mathrm{P}}-\Delta \overline{\mathbf{M}} \dot{\xi}\right) \\
& \leq-\mathrm{K}_{1}\left\|\tanh \left(\mathbf{s}_{1}\right)\right\|^{2}+\varepsilon_{1}+\tanh ^{\mathrm{T}}\left(\mathbf{s}_{1}\right) \chi
\end{aligned}
$$

where $\mathbf{s}_{2}=f(\mathbf{V})-f(\mathbf{V})^{\mathrm{d}}$ and $\chi=\overline{\mathbf{M}}_{\mathrm{o}}^{-1}\left(\mathbf{A}^{\mathrm{T}} \mathbf{s}_{2}-\mathbf{J}_{\mathrm{s}} \gamma_{2}-\Delta \overline{\mathbf{M}} \dot{\xi}\right)$.

Step 2: Design the control law for $\mathbf{U}$. Using (4.24), the dynamic equation for $\mathbf{s}_{2}$ is obtained as follows:

$$
\begin{aligned}
\dot{\mathbf{s}}_{2} & =\dot{f}(\mathbf{V})-\dot{f}(\mathbf{V})^{\mathrm{d}} \\
& =\mathbf{U}-\dot{f}(\mathbf{V})^{\mathrm{d}}
\end{aligned}
$$

The input $\mathbf{U}$ to the auxiliary system $4.24 \mathrm{c}$, is designed to robustly compensate for $\dot{f}(\mathbf{V})$. To achieve this, we construct the following compact sets:

$$
\begin{array}{r}
\Omega_{1}=\left\{\left\|\mathbf{x}_{\mathrm{e}}^{\mathrm{d}}\right\| \leq \mathrm{C}_{1},\left\|\xi^{\mathrm{d}}\right\| \leq \mathrm{C}_{2},\left\|\dot{\xi}^{\mathrm{d}}\right\| \leq \mathrm{C}_{3},\left\|\ddot{\xi}^{\mathrm{d}}\right\| \leq \mathrm{C}_{4},\right\} \\
\Omega_{2}=\left\{\left(\mathbf{s}_{1}, \mathbf{s}_{2}\right) \mid \sum_{i=1}^{9} \ln \left(\cosh \left(\left\{\mathbf{s}_{1}\right\}_{i}\right)\right)+\sum_{i=1}^{m+3} \ln \left(\cosh \left(\left\{\mathbf{s}_{2}\right\}_{i}\right)\right) \leq \mathrm{C}_{5}\right\}
\end{array}
$$

where $\mathrm{C}_{i=1,2,3,4,5}$ are positive constants. The desired detumbling trajectory constructed in this study is bounded by known bounds which can be utilized in the selection of the constants $\mathrm{C}_{1}$ to $\mathrm{C}_{4}$ in order to satisfy (4.39a). The choice of $\mathrm{C}_{5}$ is free but will affect the controller's gain selection as discussed at the end of this subsection. Furthermore, provided that $\mathbf{s}_{1}$ and $\mathbf{s}_{2}$ are contained in the compact set $\Omega_{2}$, with the use of Corollary 1, it follows that $\chi$ and $\dot{f}(\mathbf{V})^{\mathrm{d}}$ are bounded, such that $\|\chi\| \leq \mathrm{M}_{1}$ and $\left\|\dot{f}(\mathbf{V})^{\mathrm{d}}\right\| \leq \mathrm{M}_{2}$ where $\mathbf{M}_{i=1,2}$ are positive constants (proof 
of this can be found in Appendix B. From this, the control law for $\mathbf{U}$ is proposed as:

$$
\mathbf{U}=-\mathrm{K}_{2} \tanh \left(\mathbf{s}_{2}\right)-\psi_{\mathrm{P} 2}
$$

where $\mathrm{K}_{2}$ is a positive constant and $\psi_{\mathrm{P} 2}$ is a robust compensator that is defined as [28]:

$$
\psi_{\mathrm{P} 2 i}=\hat{\mathrm{M}}_{2} \tanh \left(\frac{(m+3) \mathrm{k}_{u} \hat{\mathrm{M}}_{2}\left\{\tanh \left(\mathbf{s}_{2}\right)\right\}_{i}}{\varepsilon_{1}}\right) \quad \text { for } i=1,2,3, \ldots,(m+3)
$$

The variable $\hat{\mathrm{M}}_{2}$ is an estimate of $\mathrm{M}_{2}$ and is updated by the following update law [77]:

$$
\dot{\hat{\mathbf{M}}}_{2}=\sigma_{1}\left\|\tanh \left(\mathbf{s}_{2}\right)\right\|-\sigma_{1} \sigma_{2} \hat{\mathbf{M}}_{2}
$$

Uniform ultimate boundedness of $\mathbf{s}_{1}, \mathbf{s}_{2}$ and $\tilde{M}_{2}=M_{2}-\hat{M}_{2}$ can be concluded with the use of the following Lyapunov function candidate:

$$
\mathrm{V}_{\mathrm{P}}=\mathrm{V}_{\mathrm{P} 1}+\sum_{i=1}^{m+3} \ln \left(\cosh \left(\left\{\mathbf{s}_{2}\right\}_{i}\right)\right)+\frac{1}{2 \sigma_{1}} \tilde{\mathrm{M}}_{2}^{2}
$$

The time derivative of (4.43) along with (4.37), 4.38) and 4.40 4.42) results in the following:

$$
\begin{aligned}
\dot{\mathrm{V}}_{\mathrm{P}} \leq & -\mathrm{K}_{1}\left\|\tanh \left(\mathbf{s}_{1}\right)\right\|^{2}-\mathrm{K}_{2}\left\|\tanh \left(\mathbf{s}_{2}\right)\right\|^{2}+\varepsilon_{1}+\tanh ^{\mathrm{T}}\left(\mathbf{s}_{1}\right) \chi+\tanh ^{\mathrm{T}}\left(\mathbf{s}_{2}\right)\left(-\dot{f}(\mathbf{V})^{\mathrm{d}}-\psi_{\mathrm{P} 2}\right)-\frac{1}{\sigma_{1}} \tilde{\mathbf{M}}_{2} \dot{\hat{\mathrm{M}}}_{2} \\
\leq & -\mathrm{K}_{1}\left\|\tanh \left(\mathbf{s}_{1}\right)\right\|^{2}-\mathrm{K}_{2}\left\|\tanh \left(\mathbf{s}_{2}\right)\right\|^{2}+\varepsilon_{1}+\mathrm{M}_{1}-\tanh ^{\mathrm{T}}\left(\mathbf{s}_{2}\right) \psi_{\mathrm{P} 2}+\left\|\tanh ^{\mathrm{T}}\left(\mathbf{s}_{2}\right)\right\|\left(\mathrm{M}_{2}-\hat{\mathrm{M}}_{2}\right) \\
& +\left\|\tanh ^{\mathrm{T}}\left(\mathbf{s}_{2}\right)\right\| \hat{\mathrm{M}}_{2}-\frac{1}{\sigma_{1}} \tilde{\mathrm{M}}_{2} \dot{\hat{\mathrm{M}}}_{2} \\
\leq & -\mathrm{K}_{1}\left\|\tanh \left(\mathbf{s}_{1}\right)\right\|^{2}-\mathrm{K}_{2}\left\|\tanh \left(\mathbf{s}_{2}\right)\right\|^{2}+2 \varepsilon_{1}+\mathrm{M}_{1}+\left\|\tanh ^{\mathrm{T}}\left(\mathbf{s}_{2}\right)\right\|\left(\mathrm{M}_{2}-\hat{\mathrm{M}}_{2}\right)-\frac{1}{\sigma_{1}} \tilde{\mathrm{M}}_{2} \dot{\hat{\mathrm{M}}}_{2} \\
\leq & -\mathrm{K}_{1}\left\|\tanh \left(\mathbf{s}_{1}\right)\right\|^{2}-\mathrm{K}_{2}\left\|\tanh \left(\mathbf{s}_{2}\right)\right\|^{2}-\frac{\sigma_{2}}{2} \tilde{\mathbf{M}}_{2}^{2}+\bar{\varepsilon}
\end{aligned}
$$

where the following inequalities were utilized in (4.44):

$$
\hat{\mathbf{M}}_{2}\left\|\tanh \left(\mathbf{s}_{2}\right)\right\|-\tanh ^{\mathrm{T}}\left(\mathbf{s}_{2}\right) \psi_{\mathrm{P} 2} \leq \varepsilon_{1}
$$




$$
\begin{gathered}
\sigma_{2} \tilde{\mathrm{M}}_{2} \hat{\mathrm{M}}_{2} \leq-\frac{\sigma_{2}}{2} \tilde{\mathrm{M}}_{2}^{2}+\frac{\sigma_{2}}{2} \mathrm{M}_{2}^{2} \\
\bar{\varepsilon}=2 \varepsilon_{1}+\mathrm{M}_{1}+\frac{\sigma_{2}}{2} \mathrm{M}_{2}^{2}
\end{gathered}
$$

From (4.44), $\dot{\mathrm{V}}_{\mathrm{P}}$ can be expressed as:

$$
\begin{gathered}
\dot{\mathrm{V}}_{\mathrm{P}} \leq-\rho \overline{\mathrm{V}}+\bar{\varepsilon} \\
\overline{\mathrm{V}}=\left\|\tanh \left(\mathbf{s}_{1}\right)\right\|^{2}+\left\|\tanh \left(\mathbf{s}_{2}\right)\right\|^{2}+\tilde{\mathrm{M}}_{2}^{2}
\end{gathered}
$$

where $\rho=\min \left\{\mathrm{K}_{1}, \mathrm{~K}_{2}, \sigma_{2} / 2\right\}$. Thus, $\dot{\mathrm{V}}_{\mathrm{P}}$ is strictly negative outside the following compact set:

$$
\Omega_{3}=\left\{\overline{\mathrm{V}} \mid \overline{\mathrm{V}} \leq \frac{\bar{\varepsilon}}{\rho}\right\}
$$

From 4.49, we can conclude that $\mathbf{s}_{1}, \mathbf{s}_{2}$ and $\tilde{\mathbf{M}}_{2}$ are uniformly ultimately bounded provided that $\mathrm{V}_{\mathrm{P}}(0) \leq \mathrm{C}_{5}$ and the controller gains, $\mathrm{K}_{1}, \mathrm{~K}_{2}$ and $\sigma_{2}$ be chosen such that $\rho>\bar{\varepsilon} / \overline{\mathrm{p}}$, where $\overline{\mathrm{p}}=\min _{\mathrm{V}_{\mathrm{P}}=\mathrm{C}_{5}} \overline{\mathrm{V}}$. The size of set $\Omega_{3}$ is determined by the gains $\mathrm{K}_{1}, \mathrm{~K}_{2}, \varepsilon_{1}$ and $\sigma_{2}$, and reflects the final bound on $\mathbf{s}_{1}, \mathbf{s}_{2}$ and $\tilde{M}_{2}$. A smaller bound requires higher selection of the gains $\mathrm{K}_{1}$, $\mathrm{K}_{2}$ and $\sigma_{2}$ with a lower $\varepsilon_{1}$.

A drawback of the backstepping approach is that its recursive nature can lead to numerical instability after several steps of recursion. Furthermore, estimating the time derivative of the virtual controller can be difficult. The coordination controller presented herein only required a single level of recursion and computing the time derivative of the virtual controller is not required.

\subsubsection{Servicer's End-effector Force/Torque Limits}

The proposed detumbling strategy presented in this study addresses the problem of detumbling a non-cooperative target subjected to force/torque limits by tracking a desired detumbling trajectory, where in the desired detumbling trajectory was delineated subjected to end-effector force/torque limits. In this section, it is shown that the end-effector force/torque are bounded by a function of the servicer's control input magnitude constraints and the inertial properties of 
the servicer and target. This is achieved by utilizing the servicer's equation of motion, (2.37), and the target's equation of motion, 2.42, to obtain a relationship between the servicer's control inputs and the force/torque experienced at the end-effector. The servicer's equation of motion from 2.37) is expanded as follows:

$$
\begin{aligned}
& \mathbf{M}_{11} \dot{\boldsymbol{v}}_{\mathrm{e}}+\mathbf{M}_{21} \dot{\mathbf{w}}_{\mathrm{b}}+\overline{\mathbf{c}}_{\mathrm{e}}=\tau_{\mathrm{m}}+\mathbf{J}_{\mathrm{e}}\left(-\mathbf{f}_{\mathrm{e}}\right) \\
& \mathbf{M}_{12} \dot{\boldsymbol{v}}_{\mathrm{e}}+\mathbf{M}_{22} \dot{\mathbf{w}}_{\mathrm{b}}+\overline{\mathbf{c}}_{\mathrm{b}}=\tau_{\mathrm{b}}+\mathbf{J}_{\mathrm{R}}\left(-\mathbf{f}_{\mathrm{e}}\right)
\end{aligned}
$$

where:

$$
\mathbf{M A}=\left[\begin{array}{ll}
\mathbf{M}_{11} & \mathbf{M}_{12} \\
\mathbf{M}_{21} & \mathbf{M}_{22}
\end{array}\right] \quad\left(\mathbf{M} \overline{\mathbf{c}}_{\mathrm{A}}+\mathbf{c}\right)=\left[\begin{array}{c}
\overline{\mathbf{c}}_{\mathrm{e}} \\
\overline{\mathbf{c}}_{\mathrm{b}}
\end{array}\right] \quad\left(\mathbf{J}-\mathbf{M} \mathbf{J}_{\mathrm{A}}\right)=\left[\begin{array}{l}
\mathbf{J}_{\mathrm{e}} \\
\mathbf{J}_{\mathrm{R}}
\end{array}\right] .
$$

The servicer's base angular acceleration can be eliminated from (4.50a) with the use of 4.50b, resulting in:

$$
\overline{\mathbf{J}}_{1 \mathrm{e}} \mathbf{f}_{\mathrm{e}}=\left[\left(\tau_{\mathrm{m}}-\mathbf{M}_{12} \mathbf{M}_{22}^{-1} \tau_{\mathrm{b}}\right)-\mathbf{M}_{1 \mathrm{e}} \dot{\boldsymbol{v}}_{\mathrm{e}}+-\overline{\mathbf{c}}_{1 \mathrm{e}}\right]
$$

where $\mathbf{M}_{1 \mathrm{e}}=\mathbf{M}_{11}-\mathbf{M}_{12} \mathbf{M}_{22}^{-1} \mathbf{M}_{21}, \overline{\mathbf{c}}_{1 \mathrm{e}}=\left(\overline{\mathbf{c}}_{\mathrm{e}}-\mathbf{M}_{12} \mathbf{M}_{22}^{-1} \overline{\mathbf{c}}_{\mathrm{b}}\right)$ and $\overline{\mathbf{J}}_{1 \mathrm{e}}=\left(\mathbf{J}_{\mathrm{e}}-\mathbf{M}_{12} \mathbf{M}_{22}^{-1} \mathbf{J}_{\mathrm{R}}\right)$. The servicer's end-effector acceleration can be eliminated from (4.51) with the use of the target's equation of motion in (2.42), and will result in the following:

$$
\left(\overline{\mathbf{J}}_{1 \mathrm{e}}+\mathbf{M}_{1 \mathrm{e}} \Lambda_{\mathrm{t}}^{-1}\right) \mathbf{f}_{\mathrm{e}}=\left(\tau_{\mathrm{m}}-\mathbf{M}_{12} \mathbf{M}_{22}^{-1} \tau_{\mathrm{b}}\right)+\left(\mathbf{M}_{1 \mathrm{e}} \Lambda_{\mathrm{t}}^{-1}\left(\mathbf{J}_{\mathrm{t}}^{-\mathrm{T}} \mathbf{c}_{\mathrm{t}}-\Lambda_{\mathrm{t}} \dot{\mathbf{J}}_{\mathrm{t}} \mathbf{J}_{\mathrm{t}}^{-1} \boldsymbol{v}_{\mathrm{e}}\right)-\overline{\mathbf{c}}_{1 \mathrm{e}}\right)
$$

Making use of $\Lambda_{\mathrm{t}}, \mathbf{J}_{\mathrm{t}}$ and $\mathbf{c}_{\mathrm{t}}$ defined in Section 2.6 and $h$ defined in 4.29b), it can be verified that $\left\|\mathbf{J}_{\mathrm{t}}^{-\mathrm{T}} \mathbf{c}_{\mathrm{t}}-\Lambda_{\mathrm{t}} \dot{\mathbf{J}}_{\mathrm{t}} \mathbf{J}_{\mathrm{t}}^{-1} \boldsymbol{v}_{\mathrm{e}}\right\| \leq h\left\|\mathbf{w}_{\mathrm{e}}\right\|^{2}$ similar to the derivation of the upper bound on $\gamma_{1}$ in 4.27a). Furthermore, the magnitude constraints of the servicer's control inputs results in the following upper bound: $\left\|\left(\tau_{\mathrm{m}}-\mathbf{M}_{12} \mathbf{M}_{22}^{-1} \tau_{\mathrm{b}}\right)\right\| \leq\left(\overline{\mathrm{U}}_{\mathrm{Mm}}+\left\|\mathbf{M}_{12}\right\|\left\|\mathbf{M}_{22}^{-1}\right\| \overline{\mathrm{U}}_{\mathrm{Mb}}\right)$ where $\overline{\mathrm{U}}_{\mathrm{Mm}}$ and $\overline{\mathrm{U}}_{\mathrm{Mb}}$ are the upper bounds on the servicer's manipulator and base attitude control input, respectively. Making use of this in 4.52, the end-effector's force/torque can be upper bounded as follows:

$$
\left\|\mathbf{f}_{\mathrm{e}}\right\| \leq \frac{\left(\overline{\mathrm{U}}_{\mathrm{Mm}}+\left\|\mathbf{M}_{12}\right\|\left\|\mathbf{M}_{22}^{-1}\right\| \overline{\mathrm{U}}_{\mathrm{Mb}}\right)+\left(\left\|\mathbf{M}_{1 \mathrm{e}}\right\|\left\|\Lambda_{\mathrm{t}}^{-1}\right\| h\left\|\mathbf{w}_{\mathrm{e}}\right\|^{2}+\left\|\overline{\mathbf{c}}_{\mathrm{e}}\right\|\right)}{\left(\left\|\overline{\mathbf{J}}_{1 \mathrm{e}}\right\|+\left\|\mathbf{M}_{1 \mathrm{e}}\right\|\left\|\Lambda_{\mathrm{t}}^{-1}\right\|\right)}
$$


where $\left\|\overline{\mathbf{c}}_{1 \mathrm{e}}\right\|$ if a function of known servicer's inertial and geometric parameters as well as servicer's manipulator joint and base velocities. All terms in (4.53) are based on known servicer's inertial parameters and geometry with the exception of $\left\|\Lambda_{\mathrm{t}}^{-1}\right\|$ and $h .\left\|\Lambda_{\mathrm{t}}^{-1}\right\|$ and $h$ can be estimated based on pre-launched data of the target. Furthermore, the non-linear velocity dependent terms in (4.53) can be considered negligible due to the slow rotation rate of the target [40, 53, 63], and for targets with sufficiently large inertia, the second term in the denominator of 4.53 can be approximated as $\left\|\mathbf{M}_{1 \mathrm{e}}\right\|\left\|\Lambda_{\mathrm{t}}^{-1}\right\| \approx 0$ so that the above inequality can be approximated to:

$$
\left\|\mathbf{f}_{\mathrm{e}}\right\| \leq \frac{\left(\overline{\mathrm{U}}_{\mathrm{Mm}}+\left\|\mathbf{M}_{12}\right\|\left\|\mathbf{M}_{22}^{-1}\right\| \overline{\mathrm{U}}_{\mathrm{Mb}}\right)}{\left\|\overline{\mathbf{J}}_{1 \mathrm{e}}\right\|} .
$$

The inequality in (4.53) will be evaluated with and without the inclusion of the non-linear velocity dependent terms in the numerical simulation section of this Chapter.

\subsection{Numerical Simulation Study of Detumbling Strategy}

In this subsection, numerical simulations are presented to evaluate the effectiveness of the proposed detumbling strategy to detumble a non-cooperative target with unknown but bounded inertial parameters. The evaluation is carried out using a 7-degree-of-freedom (DOF) space manipulator servicer satellite that is based on a modified model of ETS-VII system. The model parameters of the servicer are described in Appendix A. The target is modelled as a cube with $1.3 \mathrm{~m}$ sides and its inertia parameters are presented in Table 4.1. A description on how the target's inertial parameters were obtained can be found in Appendix A For the numerical simulation, the target will be modelled with zero fuel and its inertial parameters will reflect that of the Dry Condition in Table 4.1 .

The target is grasped at $t=0 \mathrm{~s}$, resulting in a redistribution of its momentum in the servicer-

Table 4.1: Target's Properties for Numerical Simulation Studies

\begin{tabular}{ccc} 
Condition: & Dry & Wet \\
\hline$m_{\mathrm{t}}, \mathrm{kg}$ & 119 & 170 \\
$\mathbf{I}_{\mathrm{t}}, \mathrm{kgm}^{2}$ & $\operatorname{diag}([32.2,32.2,33.2])$ & $\operatorname{diag}([39.8,39.8,36.3])$ \\
$\left\|\mathbf{r}_{\mathrm{te}}\right\|, \mathrm{m}$ & 1.08 & 1.15 \\
\hline
\end{tabular}


target system. The velocities of the servicer-target system after grasping $\left(t^{+}=0 \mathrm{~s}\right)$ are computed as in [67]. The target has an initial angular velocity of $\mathbf{w}_{\mathrm{t}}=[5,5,8.2]^{\mathrm{T}} \mathrm{deg} / \mathrm{s}$ at the start of the post grasping phase. The initial velocity of the target in the post-grasping phase is reasonable as ground based observation of ADEOS 1 described its attitude motion as composed of two rotational components: $0.1 \mathrm{deg} / \mathrm{s}$ about the satellite's main body and another about the satellite's boom of about 0.4deg/s [77]. Observation of Envisat resulted in an average rotation rate of 3.5deg/s [62]. Furthermore, functions from the SpaceDyn toolbox [69] were utilized to compute the servicer's mass matrix, Jacobian and nonlinear forces.

Detumbling of the target is accomplished by first determining the detumbling period utilizing bounds on the target's inertial parameters (Table A.6 of Appendix A) and the specified end-effector's force torque limit $\left(\mathrm{F}_{\max }=10 \mathrm{~N}\right.$ and $\left.\tau_{\max }=10 \mathrm{Nm}\right)$. The bounds on the target's inertial parameters presented in Appendix $\mathrm{A}$ are reflective of the target when it is full with fuel. Furthermore, in determining the detumbling period, the nonlinear velocity dependent terms in the target's equation of motion are neglected due to the slow rotation rate of the target and results in a detumbling period of $18.4 \mathrm{sec}$. Using the obtained detumbling period, the desired detumbling trajectory is delineated utilizing (4.3), (4.5) and (4.6). Tracking the reference detumbling trajectory with the unknown target attached to the servicer's end-effector is accomplished by applying the auxiliary control input $\mathbf{U}$ in 4.40 to the augmented model for the servicer's input defined in 4.24c . This provides us with the variable $\mathbf{V}$ which is utilized to compute $\tau=f(\mathbf{V})$ where $f(\mathbf{V})$ is defined in 4.24a).

The proposed detumbling strategy is presented for two different cases. In Case 1, the servicer's base control torques are realized with continuous torques applied to its center of mass. In Case 2, the servicer's base control torques are achieved with reaction thrusters that provide only on-off thrust. Case 2 is presented to demonstrate that the proposed detumbling strategy can also be incorporated with a pulse-width pulse-frequency (PWPF) modulator to control the servicer's base using on-off thrusters located on the servicer's base. As described in Section 3.4.7, from Fig. 3.7, the PWPF modulator is a feedback loop containing a linear first-order filter and Schmidt trigger and are characterized by the user-defined positive parameters: $\mathrm{K}_{m}$, $\mathrm{T}_{m}, \mathrm{U}_{\mathrm{on}}, \mathrm{U}_{\mathrm{off}}$ and $\mathrm{Y}_{\max }$ [25, 50]. Let $\Sigma_{\mathrm{b}}$ denote the body fixed frame located at the servicer's base center of mass. The components of the base attitude control torque in $\Sigma_{\mathrm{b}}$ are inputs to the PWPF modulator. The outputs of the PWPF modulator are used to fire a set of thrusters based on the thruster configuration in Fig. 3.8 and the thruster selection logic in Table 3.2 in order to apply a torque to the servicer's base. The thrusters are assumed to have a specific impulse 
Table 4.2: Gains

\begin{tabular}{ccc} 
Gain Set & $\mathrm{K}_{1}$ & $\mathrm{~K}_{2}$ \\
\hline 1 & 10 & 15 \\
2 & 10 & 40 \\
3 & 15 & 40
\end{tabular}

of $I_{s p}=70 \mathrm{~s}$ and generate an on-off reaction force of $6 \mathrm{~N}$. Furthermore, in Fig. 3.8 the radial distance, $r$ is set to $0.5 \mathrm{~m}$. A reaction force of $6 \mathrm{~N}$ is a reasonable as thrusters with similar values have been utilized with orbiting satellites [36]. Furthermore, this produces a torque of $6 \mathrm{Nm}$ about the servicer's base center of mass which is reasonable as reaction torque levels ranging between $0.01 \mathrm{Nm}$ and $30 \mathrm{Nm}$ are common in most spacecraft using reaction thrusters for attitude control [56].

The following controller gains are utilized for both Cases 1 and 2: $\varepsilon_{1}=1, \sigma_{1}=20, \sigma_{2}=3$, $\left\{\mathbf{U}_{\mathrm{M}}\right\}_{i}=8 \mathrm{Nm}$ for $i=1,2,3, \ldots 7$ (manipulator) and $\left\{\mathbf{U}_{\mathrm{M}}\right\}_{i}=6 \mathrm{Nm}$ for $i=8,9,10$ (base attitude control). Furthermore, for the detumbling task, the desired position of the end-effector is ignored and is reflected as: $\mathbf{K}_{P E L}=0 \mathbf{E}_{3}, \mathrm{~K}_{P E A}=0, \mathrm{~K}_{P B A}=0$. Numerical results are presented for Case 1 utilizing all three Gain Sets for $\mathrm{K}_{1}$ and $\mathrm{K}_{2}$ presented in Table 4.2. Case 2 sets $\mathrm{K}_{1}=4, \mathrm{~K}_{2}=10$, and the PWPF modulator parameters are $\mathrm{K}_{m}=2, \mathrm{~T}_{m}=1, \mathrm{U}_{\mathrm{on}}=0.01$, $\mathrm{U}_{\text {off }}=0.6 \mathrm{U}_{\text {on }}$ and $\mathrm{Y}_{\max }=4$.

The desired end-effector velocity and acceleration are presented in Fig. 4.1. In Figs. 4.2. 4.4. for Case 1, the velocity tracking errors are observed to decrease as gains $\mathrm{K}_{1}$ and $\mathrm{K}_{2}$ are increased from Gain Set 1 to Gain Set 3 (Table 4.2). The increase in gains from Gain Set 1 to Gain Set 3 (Table 4.2) for Case 1 do not cause the end-effector's force or torque to exceed the specified force and torque limits $\left(\mathrm{F}_{\max }=10 \mathrm{~N}, \tau_{\max }=10 \mathrm{Nm}\right)$ utilized to delineate the desired detumbling trajectory as observed in Fig. 4.5. Furthermore, the end-effector force/torque do not exceed the end-effector force/torque upper bound, as seen in Fig. 4.5. The computed force/torque upper bound in Fig. 4.5 was determined using (4.53) while neglecting the upper bound on the non-linear velocity dependent terms, $\left\|\left(\mathbf{M}_{\mathrm{e}} \Lambda_{\mathrm{t}}^{-1}\left(\mathbf{J}_{\mathrm{t}}^{-\mathrm{T}} \mathbf{c}_{\mathrm{t}}-\Lambda_{\mathrm{t}} \dot{\mathbf{J}}_{\mathrm{t}} \mathbf{J}_{\mathrm{t}}^{-1} \boldsymbol{v}_{\mathrm{e}}\right)-\overline{\mathbf{c}}_{\mathrm{e}}\right)\right\|$, in the numerator of (4.53). Note that the force/torque upper bound is less than the force/torque limits utilized to determine the detumbling period for the numerical simulations. This was done on purpose to demonstrate the controller's ability to detumble the target even if the servicer's control inputs become saturated.

The manipulator and base attitude control inputs are presented in Fig. 4.6 for Case 1 using 
gains from Gain Set 3 (Table 4.2) and can be observed to be bounded by their respective input
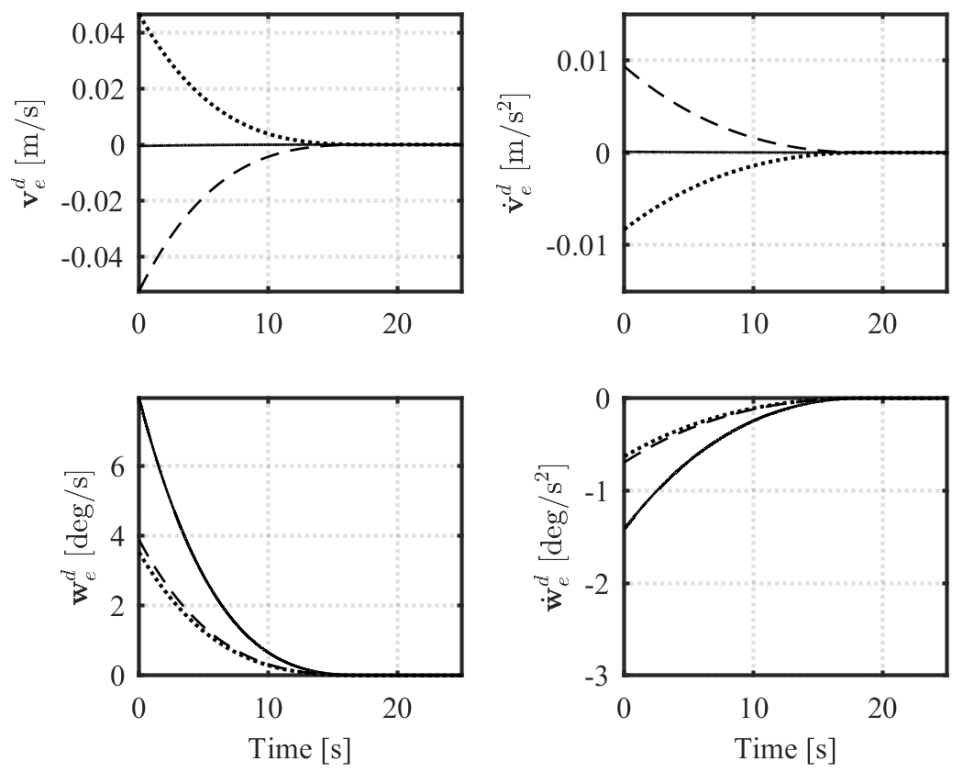

Figure 4.1: End-effector's desired linear and $\bar{y}$ angular velocity and acceleration.
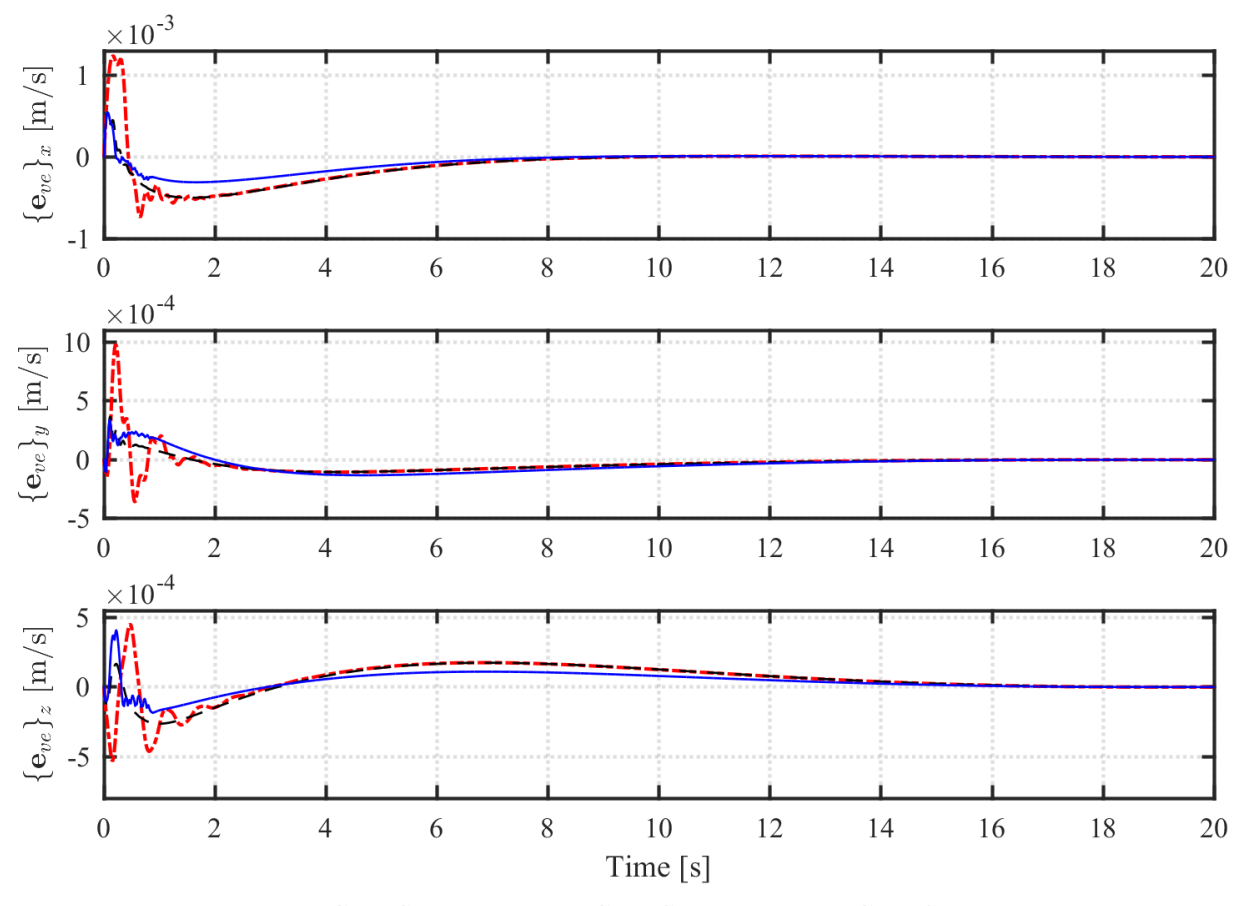

Gain Set $1----$ Gain Set 2 Gain Set 3

Figure 4.2: End-effector's linear velocity error for Case 1 


\subsection{NUMERICAL SIMULATION STUDY OF DETUMBLING STRATEGY}
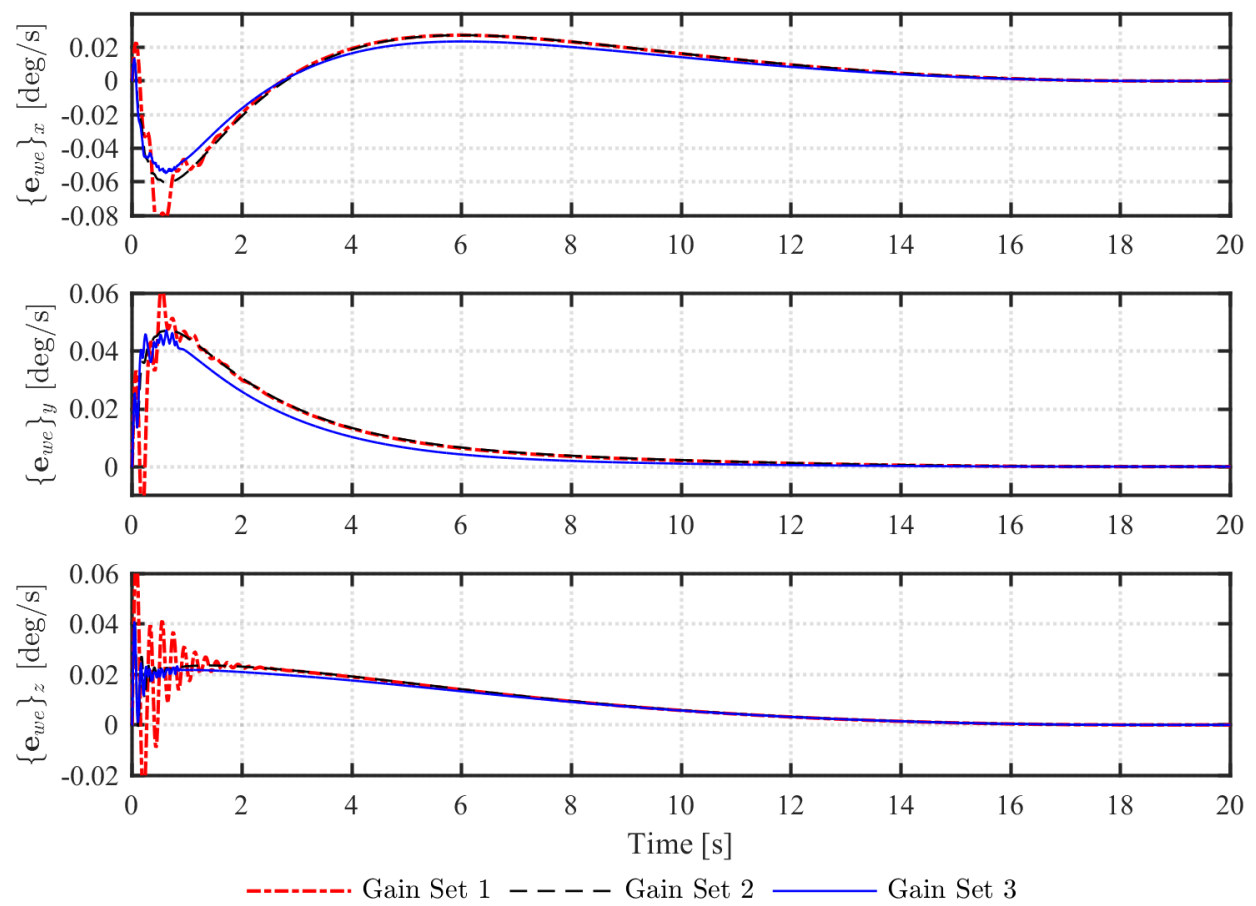

Figure 4.3: End-effector's angular velocity error for Case 1
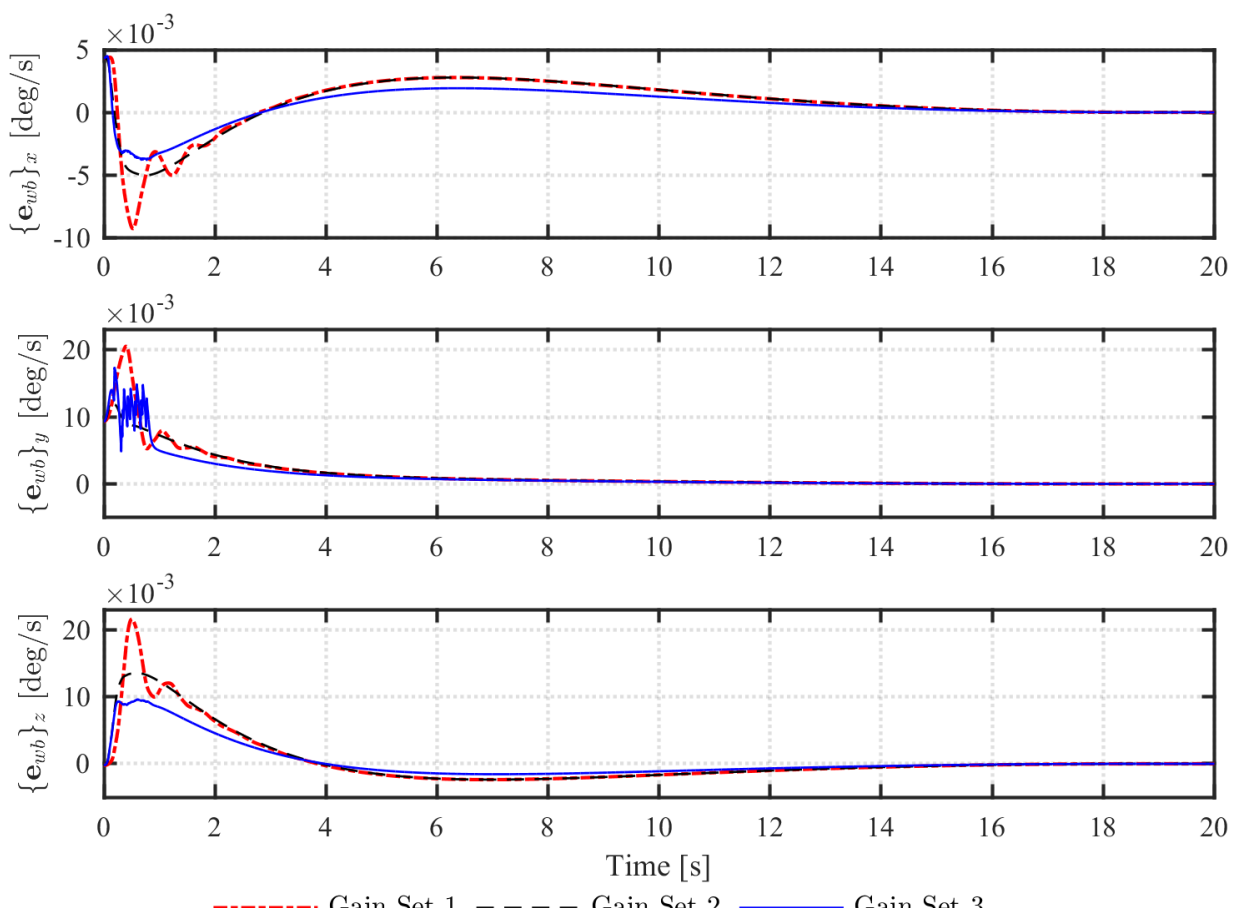

Gain Set $1----$ Gain Set 2 Gain Set 3

Figure 4.4: Servicer's base angular velocity error for Case 1 


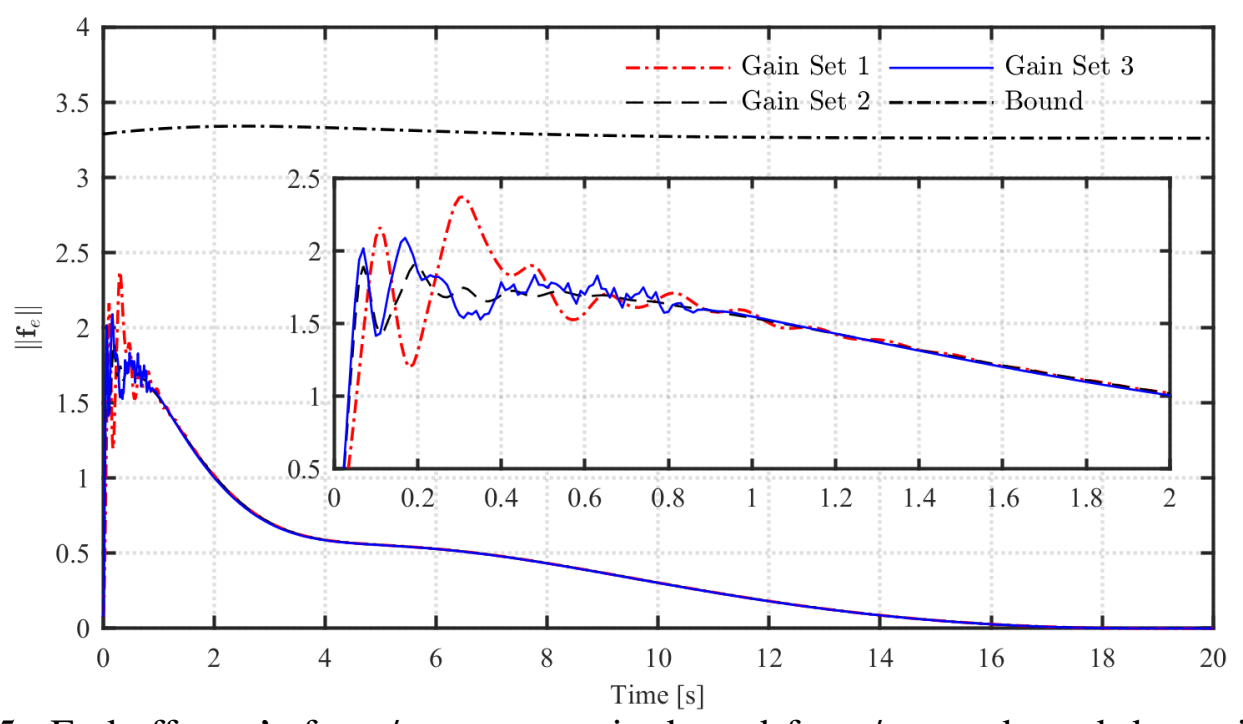

Figure 4.5: End-effector's force/torque magnitude and force/torque bound determined from (4.53) for Case 1
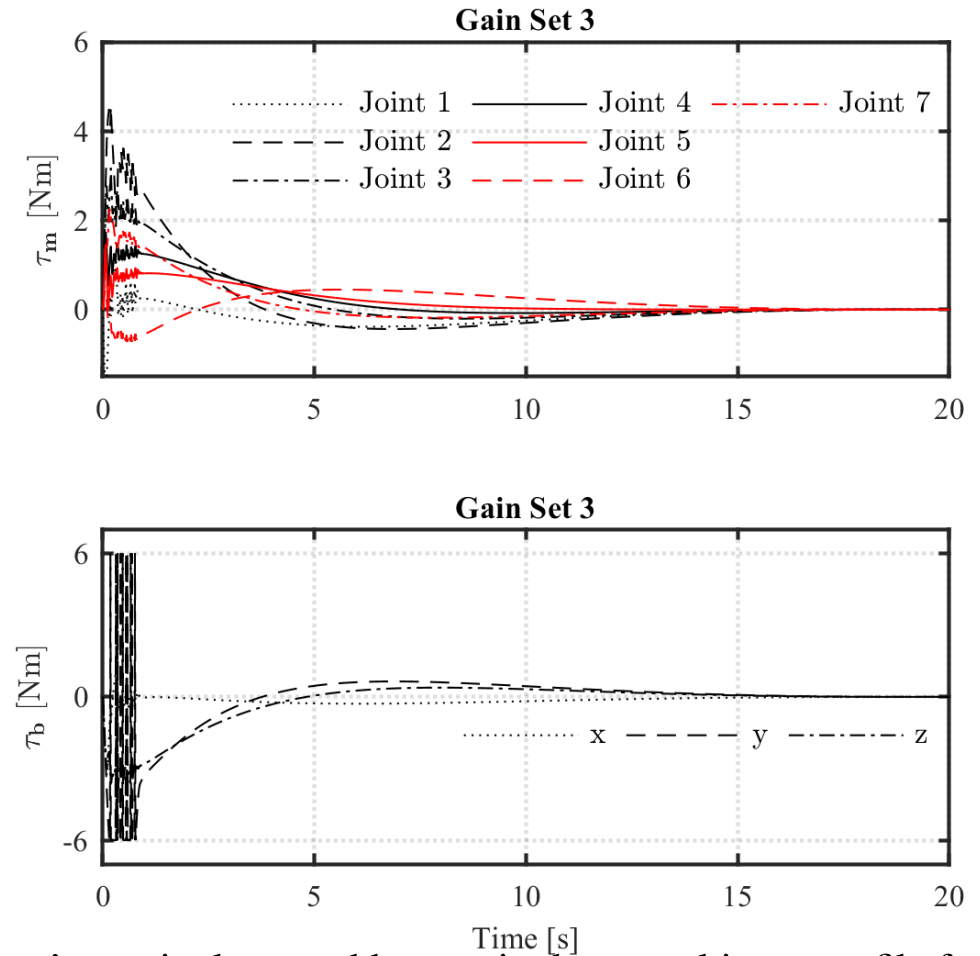

Figure 4.6: Servicer's manipulator and base attitude control input profile for Case 1, Gain Set 3 , where $\left\{\mathbf{U}_{\mathbf{M}}\right\}_{i}=8 \mathrm{Nm}$ for $i=1,2,3, \ldots 7$ (manipulator) and $\left\{\mathbf{U}_{\mathbf{M}}\right\}_{i}=6 \mathrm{Nm}$ for $i=8,9,10$ (base attitude control).

magnitude limit. It can be observed from Fig. 4.6 that detumbling of the target was accom- 
plished for Case 1, Gain Set 3, even when the base attitude control inputs were saturated.

In Case 1, detumbling of the target can be observed to be accomplished as the servicer's end-effector and base velocity errors converge to a neighbourhood of zero (Figs. 4.2 -4.4). Rejection of the target's momentum is demonstrated in Fig. 4.7. In Case 2, detumbling of the target where the base is controlled by on-off thrusters can be observed from Fig. 4.9, as the servicer's end-effector linear and angular velocity converges to a neighbourhood of zero. The end-effector's force/torque limit is observed to be respected in Fig. 4.8 while the servicer's base torque output for Case 2 is presented in Fig. 4.10. According to the classical rocket equation, the rate of change of the spacecraft's mass is determined by: $\dot{m}=\left\|\mathbf{F}_{\mathrm{c}}\right\| /\left(I_{s p} g_{\mathrm{r}}\right)$ where $\mathbf{F}_{\mathrm{c}}$ is the thruster force vector of the reaction thruster, $I_{s p}$ is the thruster's specific impulse, and $g_{\mathrm{r}}=9.81 \mathrm{~m} / \mathrm{s}^{2}$. Using this equation, the fuel consumption in Case 2 is $0.0861 \mathrm{~kg}$.
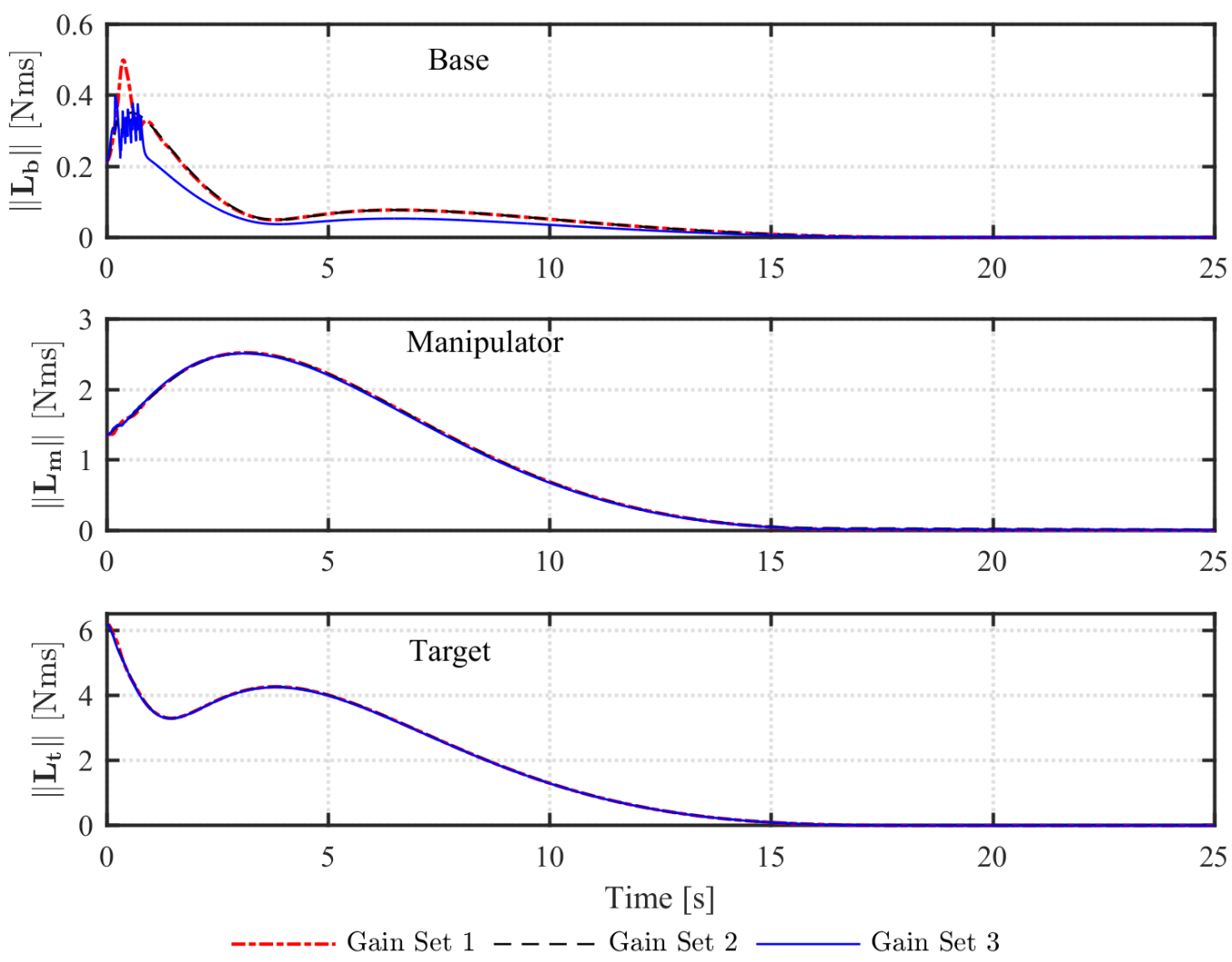

Figure 4.7: Servicer-target system's momentum distribution for Case 1. 


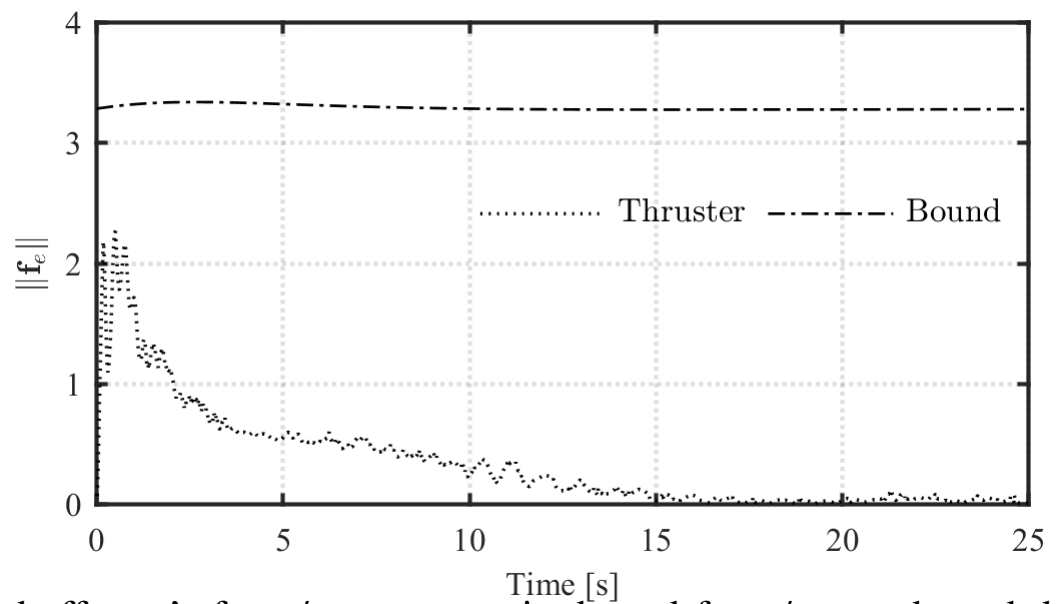

Figure 4.8: End-effector's force/torque magnitude and force/torque bound determined from 4.53) for Cases 2.
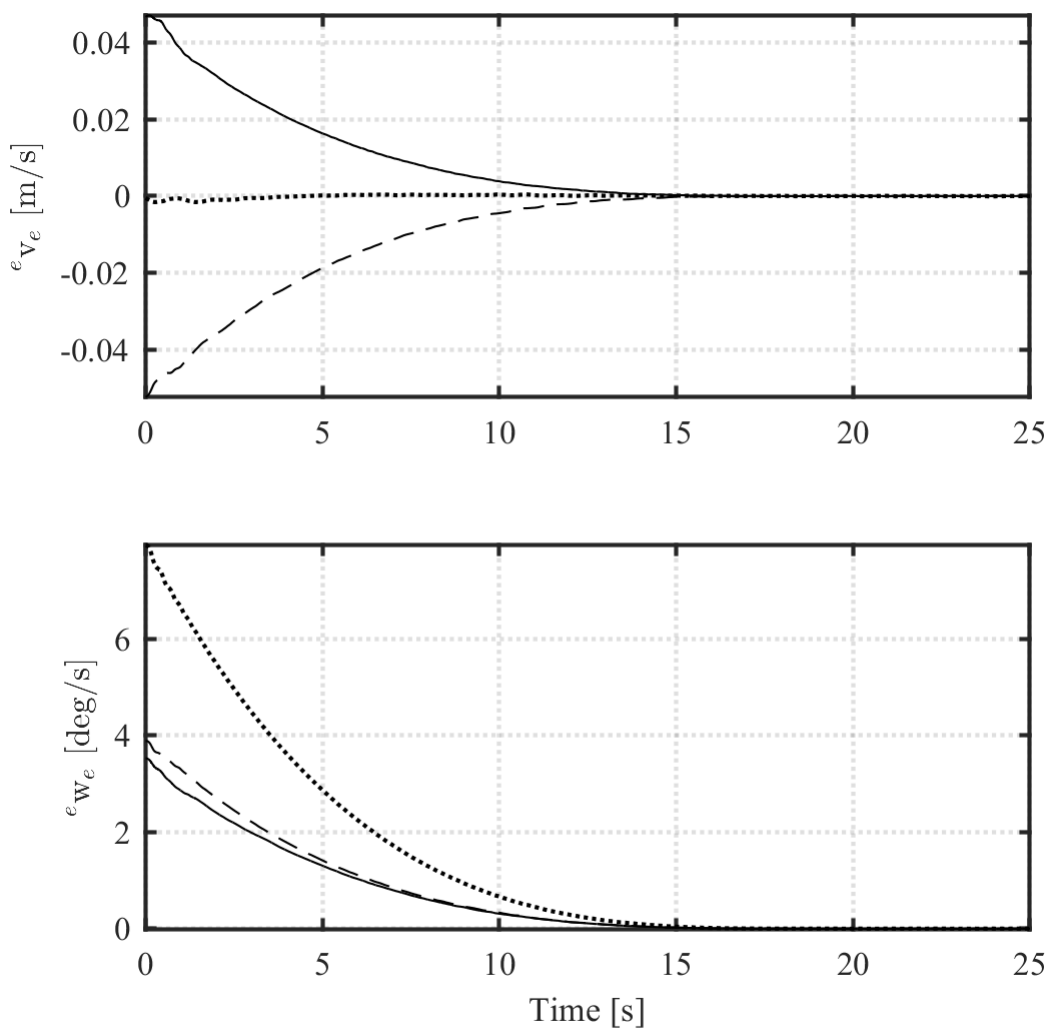

Figure 4.9: End-effector's linear and angular velocity profile for Case 2. 

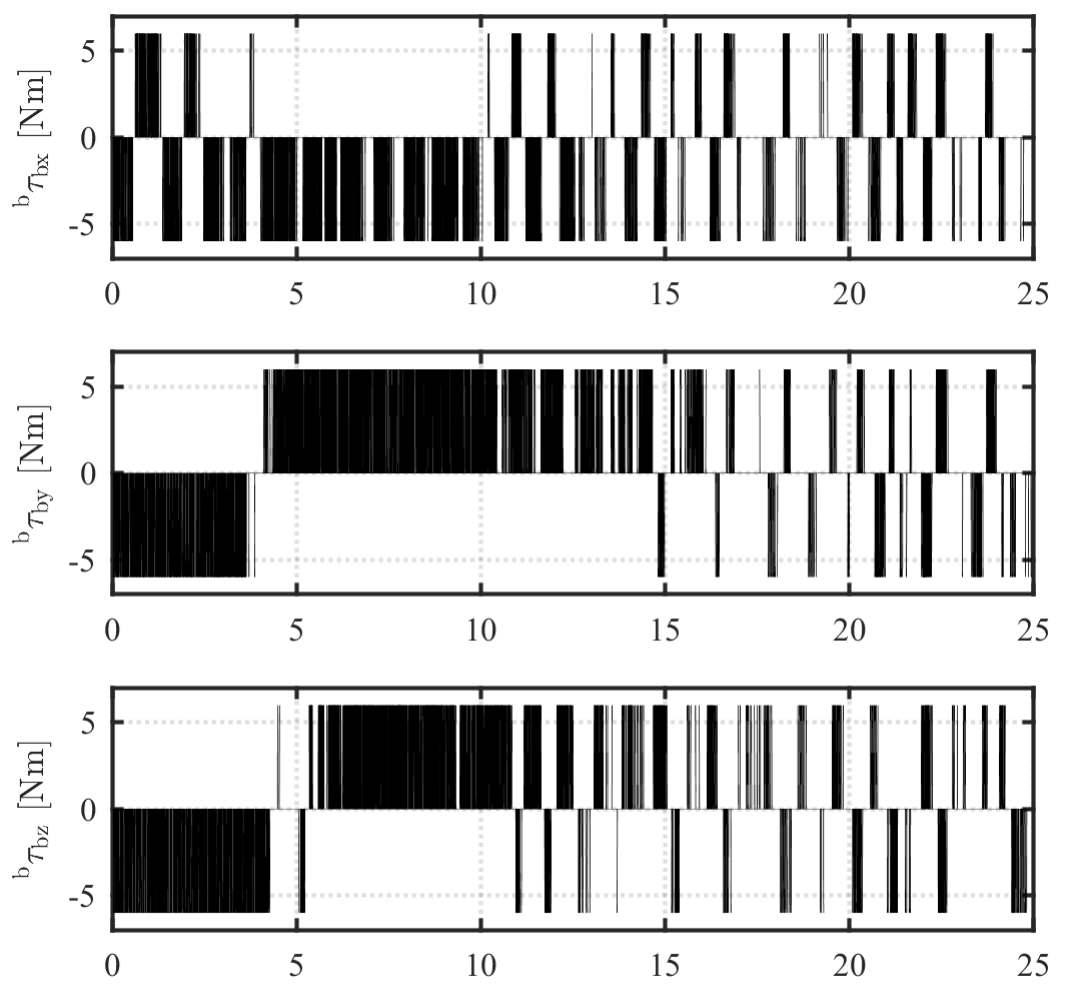

Figure 4.10: Servicer's base Torim [s] output for Case 2.

\subsubsection{Detumbling Target with Random Inertial Properties}

Let the target's dry mass, $m_{\mathrm{dry}}$, be defined as $m_{\mathrm{dry}}=119 \mathrm{~kg}$ as per Table 4.1 and denote the target's mass, $m_{\mathrm{t}}$, as the sum of its dry mass and mass of its fuel, $m_{\text {fuel }}$. From Table 4.1, the maximum fuel mass that the target can carry is $51 \mathrm{~kg}$. As the target's fuel mass varies from $0 \mathrm{~kg}$ too $51 \mathrm{~kg}$, so to does its inertial properties as described in Appendix A.

The purpose of the numerical example presented herein is to demonstrate the robustness of the proposed detumbling strategy in detumbling a target with unknown inertial parameters with known bounds. Bounds on the target's inertial parameters are determined based on its wet mass as described in Appendix A. To this end, 50 simulations are performed, where $m_{\text {fuel }}$ is randomly selected as a value between $0 \mathrm{~kg}$ and $51 \mathrm{~kg}$. The target's initial angular velocity is assumed to have the same magnitude as that in Case 1 of Section 4.2, however the direction is randomly determined as defined in 3.86 of Section 3.4.7. This is accomplished with MATLAB's "rand" function. For each numerical simulation, the servicer's base control torques are generated as 
in Case 1 of Section 4.2 (servicer's base control torques are continuous) and the detumbling control gains are the same as in Case 1 in Section 4.2 with Gain Set 2 (Table 4.2).

The main results are reported in Figs. 4.11 and 4.12. From these results, the end-effector's force/torque limits is observed to be respected, Fig. 4.11, and the target's momentum is rejected as the angular momentum of the servicer and target all converge to a neighbourhood of zero as observed in Fig. 4.12 .
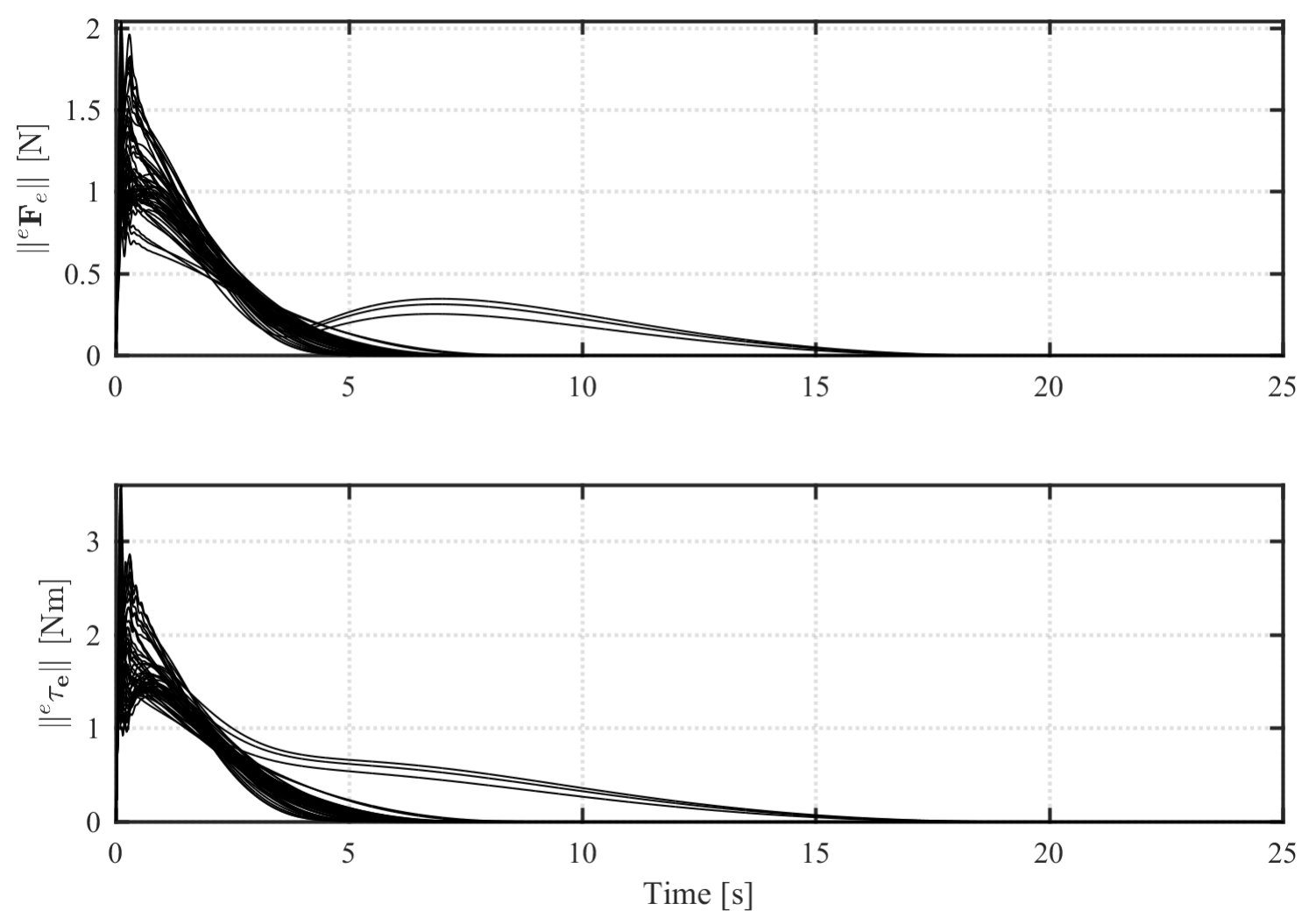

Figure 4.11: Magnitude of end-effector's force/torque profile as the target's fuel mass is randomly selected. 

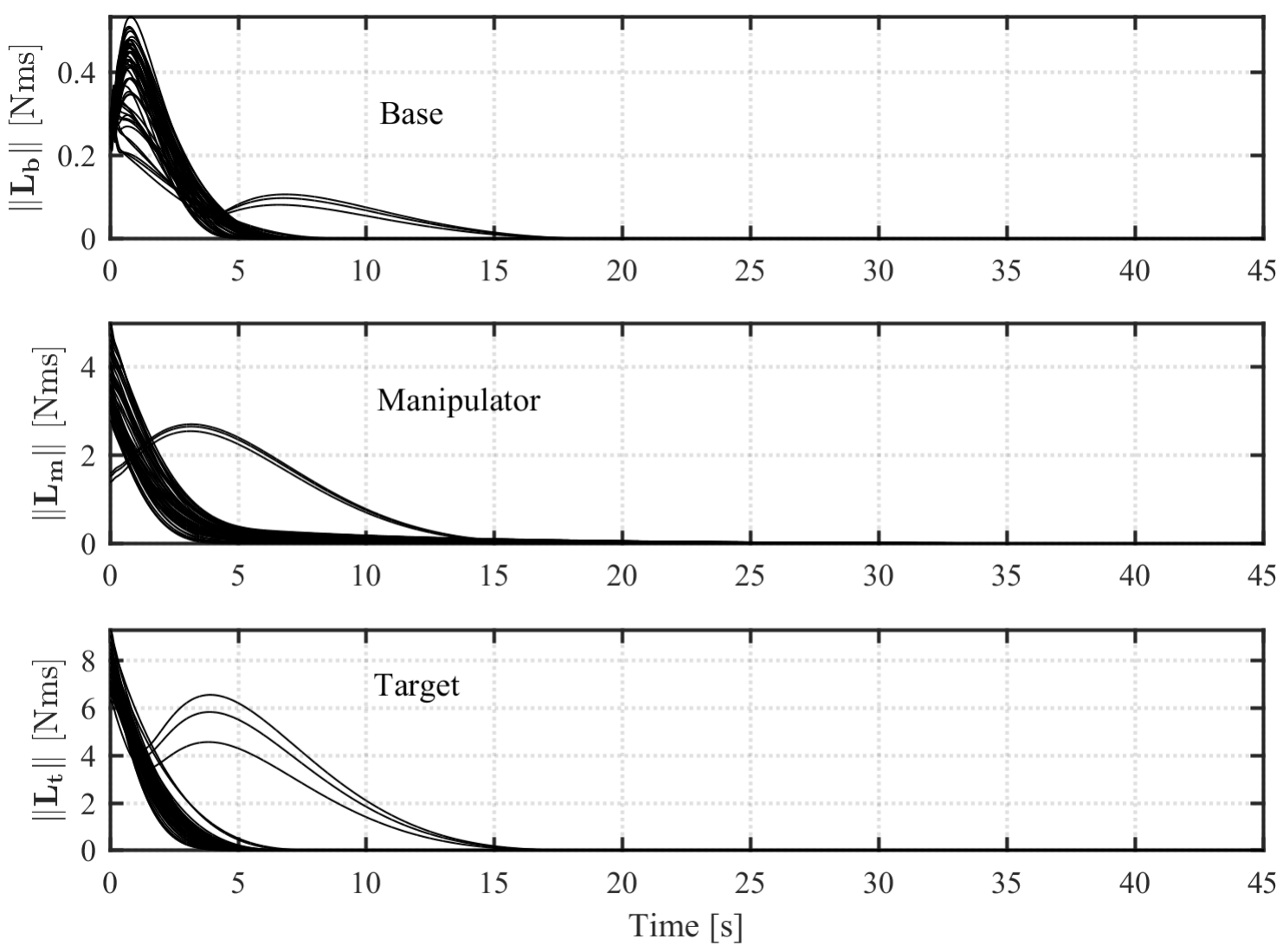

Figure 4.12: Servicer-target system's momentum distribution profile as the target's fuel mass is randomly selected.

\subsubsection{Effect of $\varepsilon_{1}$ Parameter on Detumbling Controller's Performance}

The robust compensators, $\psi_{\mathrm{P} 1}$ in 4.34 and $\psi_{\mathrm{P} 2}$ in 4.41 , were designed to robustly compensate for the disturbances preventing the sliding variable, $\mathbf{s}_{1}$ and error, $\mathbf{s}_{2}$, respectively, from converging to zero. The robust compensator can be designed using a non-smooth function (i.e., sign function) [57]. The use of a non-smooth function can lead to chattering in practical implementation of sliding mode control due to the difficulty of switching the control law at high frequency in order to keep system's dynamics on the sliding surface, and the difficulty of avoiding the excitation of unmodeled dynamics when switching the controller at high frequency [58]. Hence the smooth robust compensator used in 4.34 and 4.41 were utilized with the goal of avoiding this problem. The hyperbolic tangent function were utilized as a smooth approximation of the saturation function [57]. However, using this function requires tuning the parameter $\varepsilon_{1}$ in (4.34) and (4.41). Smaller values of $\varepsilon_{1}$ will make the hyperbolic 
tangent function better approximate the sign function as seen in Fig. 4.13,

It is observed from Fig. 4.13, as the value of $\varepsilon$ decreases the output of the hyperbolic tangent function approaches \pm 1 using a smaller argument to the hyperbolic tangent function (variable $\mathrm{t}$ in Fig. 4.13). The effect of the tuning parameter, $\varepsilon_{1}$, in $\psi_{\mathrm{P} 1}$ and $\psi_{\mathrm{P} 2}$ (defined in (4.34) and (4.41) $)$ on the detumbling controller's performance can be observed in Figs. 4.14 and 4.15. Note, the results presented in Figs. 4.14 and 4.15 were obtained utilizing Gain Set 2 in Table 4.2 and the same gains and initial conditions stated in Chapter 4.2. It is observed in Fig. 4.14 that a decrease in $\varepsilon_{1}$ corresponds to an increase in the end-effector's force and torque magnitude and even begins to oscillates. Identical observations can be made in Fig. 4.15 for the magnitude of the servicer's manipulator and base control inputs. This observations is to be expected based on the relations presented in Fig. 4.13. As the value of $\varepsilon_{1}$ is made smaller the output of the robust compenstor $\left(\psi_{\mathrm{P} 1}\right.$ and $\left.\psi_{\mathrm{P} 2}\right)$ will be larger for a smaller input. This will result in a larger control input to the servicer, and in turn, will result in a larger end-effector force/torque magnitude based on the relationship established in (4.52): larger control input will result in larger force/torque at the end-effector.

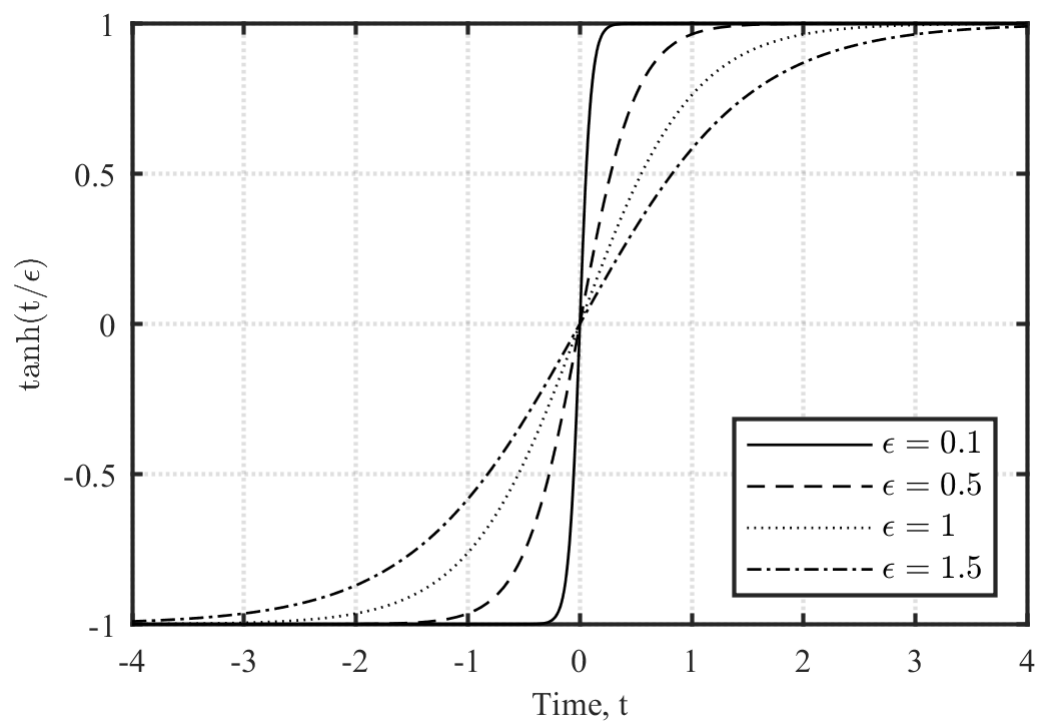

Figure 4.13: Plot of $\tanh (t / \varepsilon)$ for different values of $\varepsilon$. 

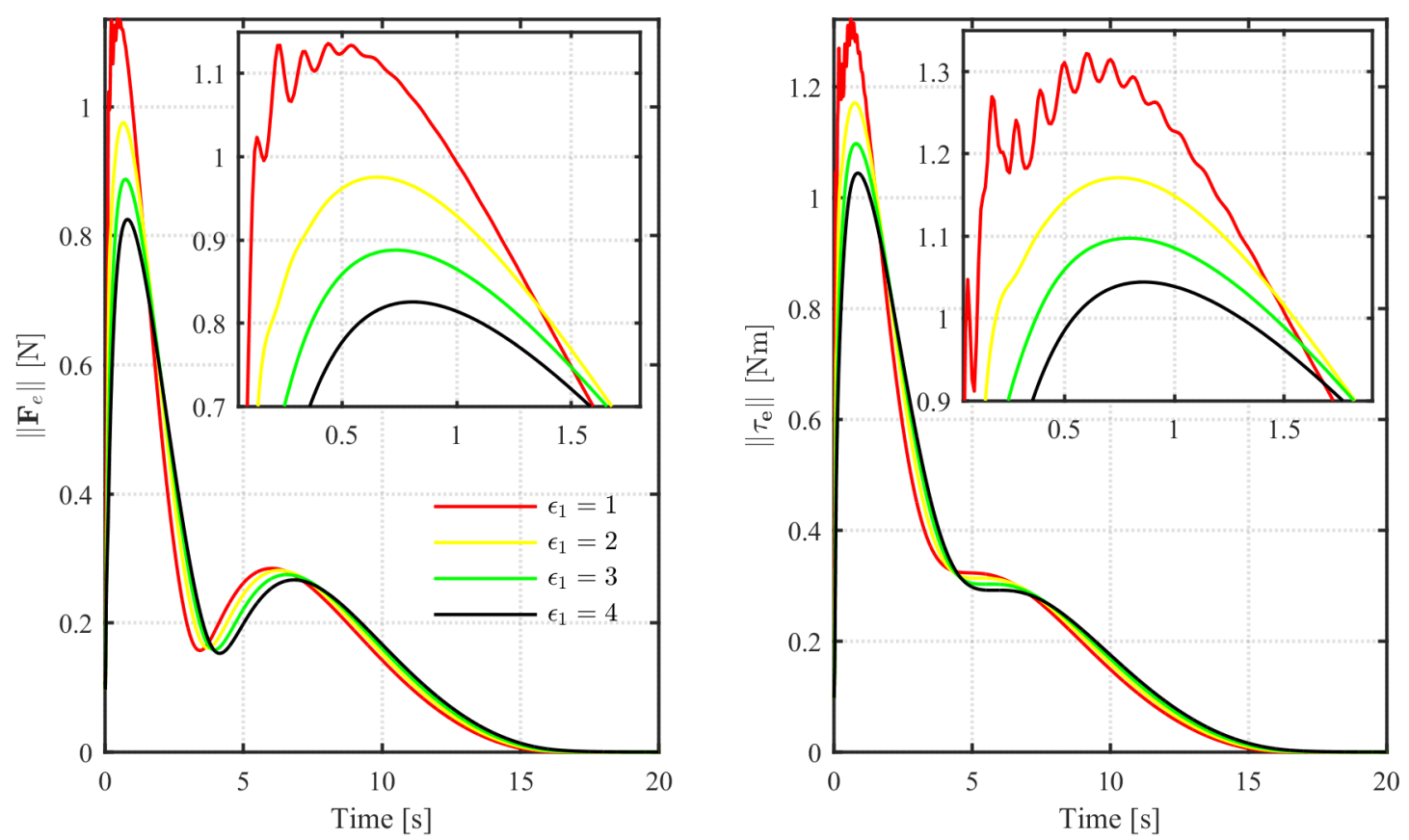

Figure 4.14: Effect of $\varepsilon_{1}$ in $\psi_{\mathrm{P} 1}$ and $\psi_{\mathrm{P} 2}$ on the end-effector force and torque magnitude profile in the post-grasping phase.
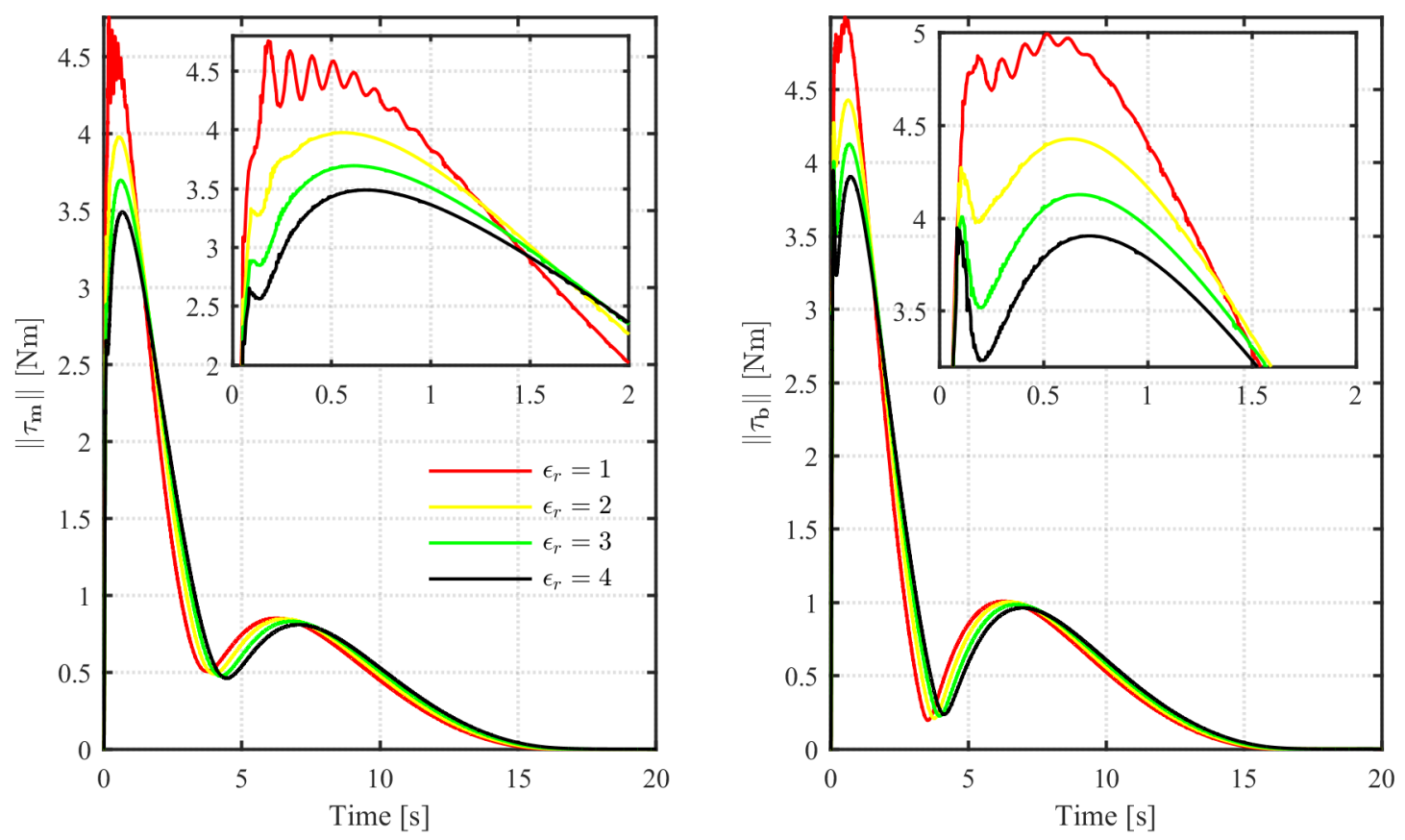

Figure 4.15: Effect of $\varepsilon_{1}$ in $\psi_{\mathrm{P} 1}$ and $\psi_{\mathrm{P} 2}$ on the magnitude of the servicer's control inputs. 


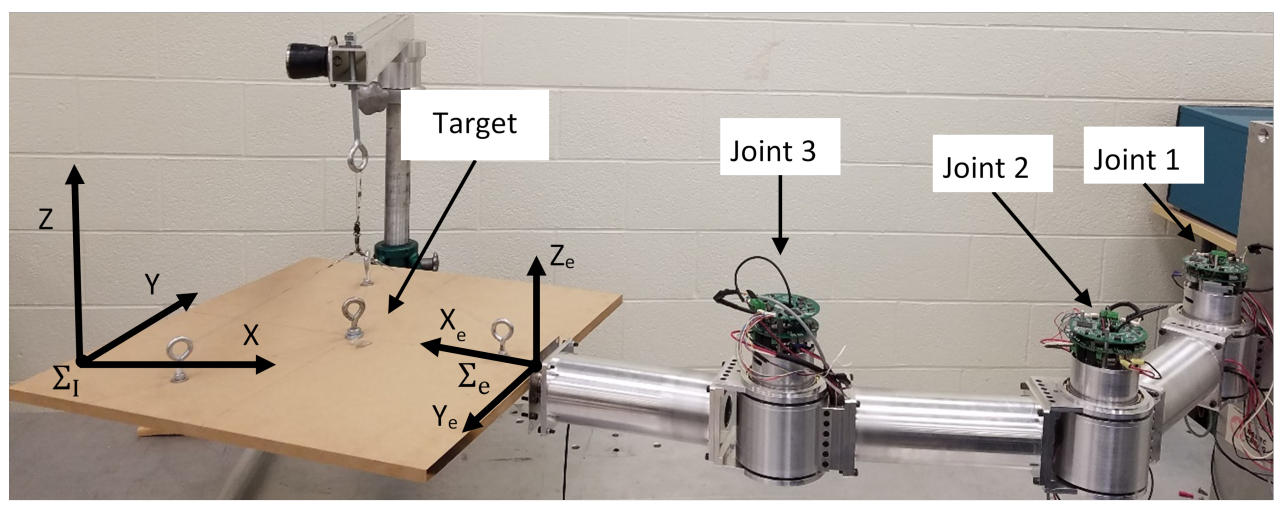

Figure 4.16: Experimental setup

\subsection{Experimental Evaluation}

Experiments are conducted to evaluate the design of the desired detumbling trajectory by comparing the specified end-effector force/torque limits to the measured end-effector force/torque. This is accomplished by tracking a desired detumbling trajectory and comparing the specified force/torque limits used to delineate the desired detumbling trajectory to the measured endeffector force/torque. The procedure for designing the desired detumbling trajectory is as follows: 1) utilize bounds on the target's inertial parameters and 2) the end-effector force/torque limits to determine the minimum detumbling period; utilize the detumbling period to delineate the desired detumbling trajectory.

The experimental setup is depicted in Fig. 4.16 and is confined to the X-Y plane. It consists of the target and a 3 degree of freedom (DOF) Modular and Reconfigurable Robot that was designed and built at the Systems and Controls Lab at Ryerson University. Each joint of the manipulator is equipped with an absolute encoder to measure the link-side position and the endeffector is equipped with a 6 axis force/torque sensor (ATI Mini45). The target is represented by a square platform constructed from MDF (Fig. 4.16) and hangs from a taut string and has the following inertial properties: $m_{t}=5 \mathrm{~kg}, \lambda_{\max }\left(\mathbf{I}_{\mathrm{t}}\right)=0.3 \mathrm{kgm}^{2}$ and $\left\|\mathbf{r}_{\mathrm{te}}\right\|=0.3 \mathrm{~m}$.

From Fig. 4.16, the manipulator's base joint is fixed and does not allow the emulation of the servicer's base motion. However, this does not effect the intended goal of the experiment. This becomes a requirement when evaluating the performance of the proposed controller in Section 4.1.3 in its ability to reject the coupling dynamics between the servicer's manipulator 
and base in order to track the desired detumbling trajectory (controller's performance not being experimentally evaluated). As a result, the desired end-effector detumbling trajectory is mapped to joint space, and a simple PD controller is implemented to track the desired joint space trajectory.

Since the target hangs from a taunt string (experimental setup in Fig. 4.16), it is undesirable for its center of mass to translate as this will induce an unwanted restoring force that is unrepresentative of a target in space. With this in mind, the detumbling trajectory is designed by forcing $\left\|\mathbf{F}_{\mathrm{e}}\right\|=\mathrm{F}_{\max }=0$ in $(4.7)$ so as to not induce any translational movement of the target's center of mass during the experiment. This experimental constraint requires $\mathbf{r}_{\text {te }}$ to be known in order to compute the desired end-effector position trajectory that will satisfy $\left\|\mathbf{F}_{\mathrm{e}}\right\|=\mathrm{F}_{\max }=0$, and results in only the end-effector's torque being evaluated along the desired detumbling trajectory. As a result, 4.7) and (4.8) reduce to the following for the planner case:

$$
\begin{gathered}
\dot{\mathbf{v}}_{\mathrm{e}}^{\mathrm{d}}=-\mathbf{r}_{\mathrm{te}}^{\times} \dot{\mathbf{w}}_{\mathrm{e}}^{\mathrm{d}}-\dot{\mathbf{r}}_{\mathrm{te}}^{\times} \mathbf{w}_{\mathrm{e}}^{\mathrm{d}} \\
\mathbf{I}_{\mathrm{t}} \dot{\mathbf{w}}_{\mathrm{e}}^{\mathrm{d}}=\tau_{\mathrm{e}}
\end{gathered}
$$

From (4.55b), the detumbling period is determined as follows:

$$
T \geq \frac{\left|\mathbf{w}_{\mathrm{tz}(\mathrm{int})}\right| \lambda_{\max }\left(\mathbf{I}_{\mathrm{t}}\right)}{\tau_{\max }}
$$

where setting $\mathbf{w}_{\mathrm{tz}(\text { int })}=5 \mathrm{deg} / \mathrm{s}, \lambda_{\max }\left(\mathbf{I}_{\mathrm{t}}\right)=1 \mathrm{kgm}^{2}$ and $\tau_{\max }=5 \mathrm{Nm}$ produces a detumbling period of $T \geq 0.02 \mathrm{sec}$. While setting $T$ to a very small value may seem valuable, it will result in the excitation of unmodelled dynamics. For the experiment, two cases will be considered. In Case $1, T=10 \mathrm{sec}$ and in Case $2, T=15 \mathrm{sec}$. Using the detumbling period, the desired detumbling trajectory of the end-effector's orientation and its first and second derivatives are computed from (4.3), (4.5) and (4.6). The desired trajectory of the end-effector's position is determined by integrating 4.55a).

The experimental procedure is partitioned into two phases. The goal of phase one is to set the initial conditions for the post-grasping phase so that the detumbling trajectory can be evaluated in phase two. Phase one starts at point A in Fig. 4.17a, and controls the manipulator's end-effector to move from point A to point B along a smooth trajectory such that the 
angular velocity of the end-effector at point $\mathrm{B}$ is equal to $\mathbf{w}_{\mathrm{tz} \text { (int) }}$ (initial angular velocity of post-grasping phase). Phase two starts once the end-effector arrives at point B in Fig. 4.17b, where tracking of the desired detumbling trajectory commences.

The manipulator joint position and velocity tracking errors are presented in Figs. 4.18 and 4.19, respectively, for both Cases 1 and 2. The measured end-effector's torque and force profile in frame $\Sigma_{\mathrm{e}}\left(\Sigma_{\mathrm{e}}\right.$ defined in Fig. 4.16) are presented in Fig. 4.20 for Cases 1 and 2. From the aforementioned plots (Figs. 4.18, 4.19, 4.20), phase one occurs from $t=0$ sec to $t=15 \mathrm{sec}$, and phase two starts at $t=15 \mathrm{sec}$ and ends at $t=25 \mathrm{sec}$ for Case 1 and $t=30 \mathrm{sec}$ for Case 2. The manipulator tracks the desired trajectory in both phases one and two (Figs. 4.18 and 4.19p. Due to the experimental setup constraint, the desired detumbling trajectory was designed to keep $\mathbf{F}_{\mathrm{e}}=0$ along the detumbling trajectory. However, from Fig. 4.20, the end-effector force is not exactly zero for both Cases 1 and 2. A non zero end-effector force would result in an addition term contributing to the measured end-effector torque in

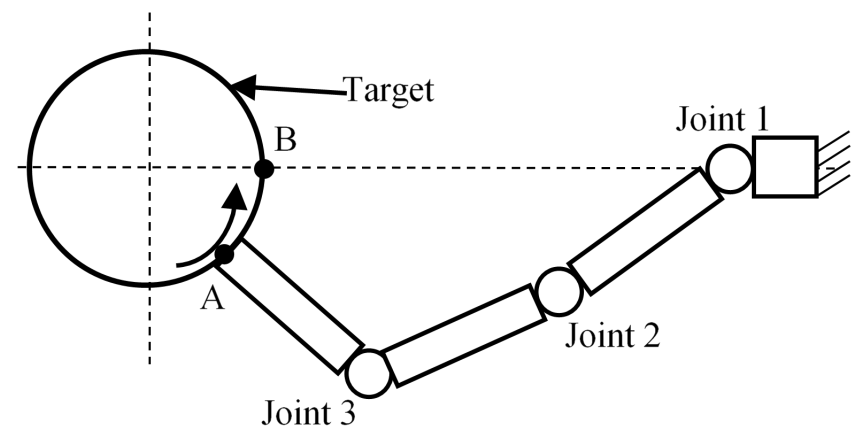

Figure 4.17a: Phase one of experiment

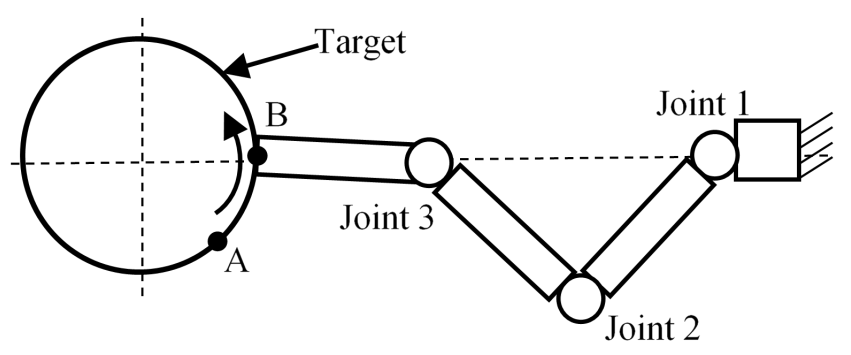

Figure 4.17b: Phase two of experiment 


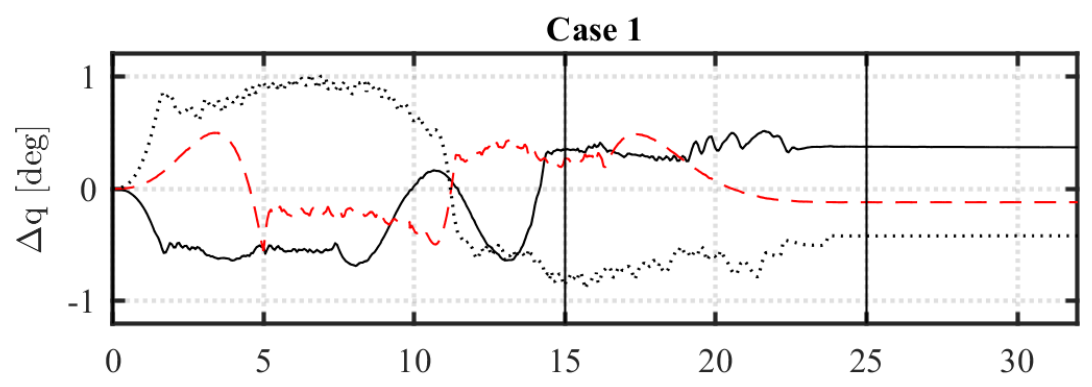

Case 2

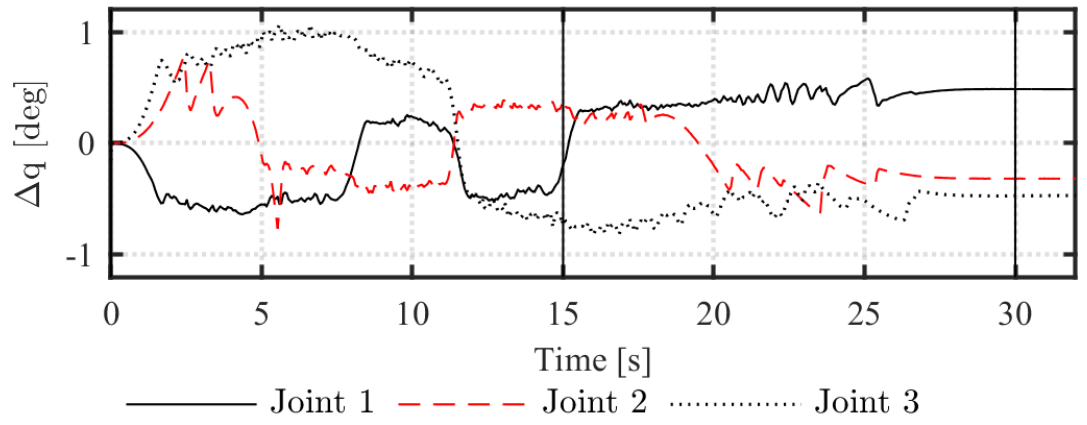

Figure 4.18: Manipulator joint position error

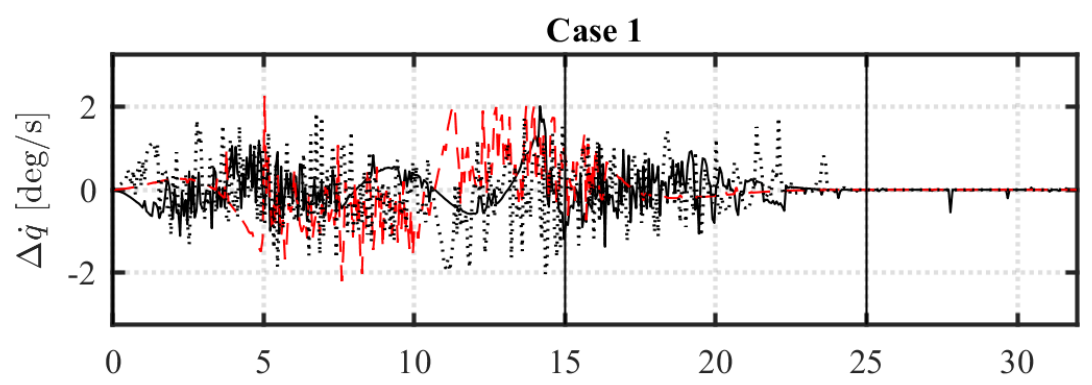

Case 2

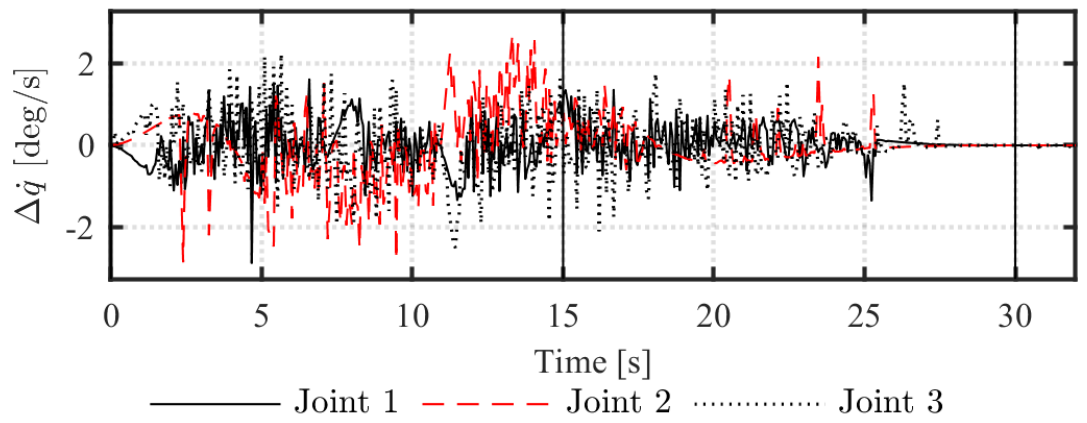

Figure 4.19: Manipulator joint velocity error 

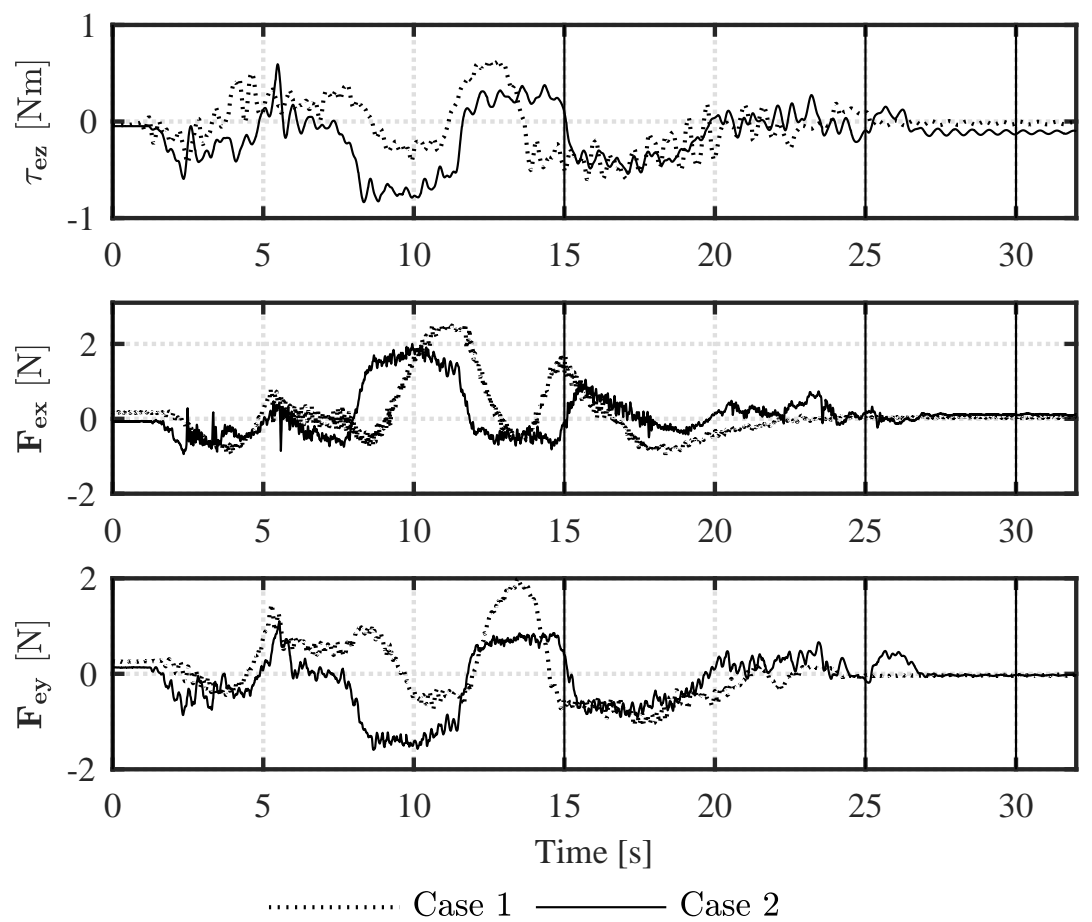

Figure 4.20: Measured end-effector's force/torque for Cases 1 and 2 in frame $\Sigma_{\mathrm{e}}$ located at the end-effector. Top plot presents torque about $\mathrm{z}$ axis of $\Sigma_{\mathrm{e}}$. Bottom two plots present force along $\mathrm{x}$ and $\mathrm{y}$ axis of $\Sigma_{\mathrm{e}}$.

addition to the inertial torque of the target along the desired detumbling trajectory: $4.55 \mathrm{~b}$ would become $-\mathbf{r}_{\mathrm{te}}^{\times} \mathbf{F}_{\mathrm{e}}+\mathbf{I}_{\mathrm{t}} \dot{\mathbf{w}}_{\mathrm{e}}=\tau_{\mathrm{e}}$. Given $\left\|\mathbf{r}_{\mathrm{te}}\right\|=0.3 \mathrm{~m}$ of the target platform in Fig. 4.16, and the maximum $\left\|\mathbf{F}_{\mathrm{e}}\right\|$ from Fig. 4.20 for $t \geq 15 \mathrm{sec}$, the maximum torque created by the additional term $\mathbf{r}_{\mathrm{te}}^{\times} \mathbf{F}_{\mathrm{e}}$ in phase 2 of the experiment $(t \geq 15 \mathrm{sec})$ is determined to be $\left\|\mathbf{r}_{\mathrm{te}}^{\times} \mathbf{F}_{\mathrm{e}}\right\|=0.54 \mathrm{Nm}$. The additional torque created by $\left\|\mathbf{r}_{\mathrm{te}}^{\times} \mathbf{F}_{\mathrm{e}}\right\|$ is well below the torque limit of $5 \mathrm{Nm}$ that was utilized to compute the desired detumbling trajectory and hence does not affect the results obtained from the experiment.

The end-effector's torque profile is presented in the top plot of Fig. 4.20 for both Cases 1 and 2. For both cases, the end-effector's measured torque is observed to have a maximum at the start of the desired detumbling trajectory $(t=15 \mathrm{sec})$ and converges to around zero as the target is brought to rest along the desired detumbling trajectory. It is clearly observed that the measured torque is well within the specified torque limit utilized to construct the desired detumbling trajectory even as the detumbling period is reduced from $T=15 \mathrm{sec}$ to $T=10 \mathrm{sec}$. 


\subsection{Conclusions}

This chapter has addressed the problem of detumbling a non-cooperative target with unknown but bounded inertial parameters by a space robot. The detumbling strategy presented within this chapter was developed under the basis that measurements of the end-effector force/torque are not available. Detumbling of the target is accomplished by designing a robust controller to coordinate the motion between the servicer's base and manipulator in order to track a desired task space detumbling trajectory and to accommodate the target's gained momentum as its tumbling motion is brought to rest. The proposed robust controller takes into account magnitude constraints on the servicer's control input in the controller's design. The desired detumbling trajectory is delineated subjected to end-effector force/torque limits and requires only bounds on the target's inertial parameters. The use of bounds on the target's inertial parameters in the proposed detumbling strategy to both determine the detumbling trajectory and to track it, eliminates the need for accurate knowledge of the target's inertial parameters, which are impractical to be assumed known prior to detumbling the target. Numerical simulations and experimental evaluations have demonstrated that the end-effector's force/torque limits are respected as the target with unknown inertial parameters is detumbled by the proposed detumbling strategy. 


\section{Chapter 5}

\section{Conclusions and Future Work}

\subsection{Summary and Conclusions}

In this dissertation, the problem of detumbling a malfunctioning satellite by a space robot for the purpose of performing on-orbit servicing is addressed. The malfunctioning satellite is denoted as the target and is non-cooperative, tumbling and has unknown but bounded inertial parameters. The space robot consists of a manipulator attached to a satellite base. Two detumbling control strategies are presented in this study. In both detumbling strategies, detumbling of the target is accomplished while being subjected to force/torque limits at the servicer's endeffector and do not require knowledge of the target's inertial parameters other than estimates of bounds on the target's inertial parameters.

The difference between the two detumbling strategies lie in the assumption on the availability of force/torque measurements at the end-effector. If end-effector force/torque measurements are not available, a tracking control based detumbling strategy is presented. Alternatively, if force/torque measurements are available, a force control based detumbling strategy is presented. The availability of force/torque measurements at the end-effector is not impossible to achieve, but adds additional complexity as the design and housing of the sensor must be taken into account for successful operation in the harsh space environment (i.e., large temperature fluctuations).

The force control based detumbling strategy achieves detumbling by applying a reference detumbling force/torque to the target that is designed to bring the target's velocity to zero subjected to force/torque limits at the servicer's end-effector, and is computed using the servicer's 
end-effector velocity and force/torque limits. This allows the servicer to detumble the target while complying with its residual tumbling motion. The implementation of this detumbling strategy is presented with and without compensation for changes in the servicer's inertial parameters due to the grasped target. In the case of no compensation for changes in the servicer's inertial parameters a detumbling criteria is formulated in the form of bounds on the target's inertial parameters and tumbling rate for which this approach can detumble (domain of attraction). Evaluation of the detumbling criteria can be carried out utilizing estimates of bounds on the target's inertial parameters.

If the estimated bounds on the target's inertial parameters are very conservative, then it may become difficult to satisfy the detumbling criteria. In this case, an adaptive robust compensator was designed to ensure stable detumbling of the target utilizing real-time estimates of bounds on the target's inertial parameters to update its gains. The design of the reference detumbling force/torque and use of real-time estimates of bounds on the target's inertial parameters eliminates the need for accurate knowledge of the target's inertial parameters and their bounds, and allows the robust controller gains be adjusted in real-time based on real-time estimates of bounds on the target's inertial parameters. Bounds on the end-effector force/torque are derived along with bounds on the servicer's control inputs. Numerical simulations have demonstrated that the end-effector force/torque limits are respected as the target with unknown inertial parameters is detumbled using the force control based detumbling strategy.

The tracking control based detumbling strategy achieves detumbling of the target by designing a robust coordination controller to coordinate the motion between the servicer's base and manipulator in order to track a desired task space detumbling trajectory and to reject the target's gained momentum. The proposed robust coordination controller takes into account magnitude constraints on the servicer's control inputs in the controller's design. The desired detumbling trajectory is delineated subjected to end-effector force/torque limits and requires only bounds on the target's inertial parameters. The use of bounds on the target's inertial parameters in the proposed detumbling strategy to both determine the detumbling trajectory and to track it eliminates the need for accurate knowledge of the target's inertial parameters, which are impractical to be assumed known prior to detumbling the target. Numerical simulations and experimental evaluations have demonstrated that the end-effector force/torque limits are respected as the target with unknown inertial parameters is detumbled using the tracking control based detumbling strategy.

The tracking control based detumbling strategy indirectly takes into account limits on the 
servicer's end-effector force/torque. Force/torque limits at the end-effector are taken into account via the design of the desired detumbling trajectory and coordination control between the servicer's manipulator and base is implemented to track the desired trajectory. However, the end-effector's force/torque experienced during the detumbling of the target is shown to be upper bounded by a function of the servicer's control input magnitude constraints in Section 4.1.4 Larger control input magnitude constraints will result in a larger force/torque bound. Hence, utilizing the servicer's control input magnitude constraints, the maximum force/torque experienced at the end-effector can be determined and specified as the end-effector's force and torque limits. These limits in conjunction with bounds on the target's inertial parameters can be utilized to delineate the desired detumbling trajectory. Design of the desired detumbling trajectory in this manner, and the utilization of the robust coordination controller to track the desired detumbling trajectory while rejecting the target's gained momentum will ensure force/torque limits at the servicer's end-effector are respected.

Both detumbling strategies are capable of detumbling the target subjected to force/torque limits at the end-effector using only bounds on the target's inertial parameters. However, there are inherent advantages and disadvantages between the two strategies. The tracking control based detumbling strategy does not require force/torque measurements at the end-effector in its implementation. While this presents an advantage, the resultant strategy produces conservative requirements on the servicer's design in order to respect end-effector force/torque limits.

The procedure to delineate the desired detumbling trajectory in the tracking control based detumbling strategy, outlined in Section 4.1.1, utilizes bounds on the target's inertial parameters to determine the detumbling period. The detumbling period is then used to delineate the desired detumbling trajectory (recall that a smaller detumbling period results in faster detumbling of the target and larger force/torque at the end-effector during the detumbling procedure). As the bounds on the target's inertial parameters are inherently larger than the target's actual inertial parameters, a larger detumbling period will result leading to experienced force/torque at the end-effector that are smaller in comparison to their respective force/torque limit.

Furthermore, the procedure to delineate the desired detumbling trajectory in the tracking control based detumbling strategy imposes constraints on the target's inertial parameters for which it can detumble based on the servicer's force/torque limits: using bounds on the target's inertial parameters and the servicer's force/torque limits, the inequality in (4.18) must be satisfied. Inequality (4.18) dictates that an increase in the target's inertial parameters and tumbling rate requires a servicer designed with a larger force/torque limit in order to arrive at a feasible 
solution for the detumbling period $(T>0)$. As a comparison, if the tracking control based detumbling strategy were to be used to detumble the target with the same inertial properties and tumbling rate as that in the numerical simulation study of the force control based detumbling strategy in Section 3.4.7, the servicer will be required to possess force and torque limits of at least $35 \mathrm{~N}$ and $35 \mathrm{Nm}$, respectively, to be able to delineate a detumbling trajectory. In Section 3.4.7, using the force control based detumbling strategy, detumbling of the target was achieved with a servicer specified with $10 \mathrm{~N}$ and $10 \mathrm{Nm}$ for force and torque limit at the end-effector, respectively. This highlights the advantage of the force control based detumbling strategy in that it would require a servicer designed with smaller force/torque limits to detumble the same target than with the use of the tracking control based detumbling strategy. A servicer designed with smaller force/torque limits would require smaller control inputs to detumble the target and reject its momentum as well as a grasping mechanism designed to handle smaller interaction force/torque at the grasping point.

\subsection{Future Work}

It is the hope of the author that the proposed detumbling control strategies have advanced the literature on the subject and stimulated ideas on how to further develop detumbling control strategies to address the problem of detumbling a non-cooperative target with unknown but bounded inertial parameters. Some of the possible future directions of research on the problem are as follows:

1. Improve the delineation of the desired detumbling trajectory in the tracking control based detumbling strategy so that the end-effector's experienced force and torque that occur during the detumbling procedure are closer to the end-effector's force/torque limits. Furthermore, the inclusion of additional considerations in the delineation of the detumbling trajectory should be considered: minimize fuel consumption, avoid singular configuration.

2. An investigation should be carried out to determine the possibility of utilizing a parameter estimation scheme with the force control based detumbling strategy in order to identify the target's inertial parameters and to predict its motion. This would enable the servicer to determine if the target will rotate out of its workspace and allows for the pos- 
sibility of corrective action by the servicer: i.e., change the direction and magnitude of the applied detumbling force/torque.

3. An investigation should be carried out to study the implementation of both detumbling control strategies using a servicer that consists of two manipulators attached to the servicer's base. One arm can be utilized to detumble the target, while the second arm can be utilized to manage the momentum distribution in the servicer system in order to avoid saturation of the servicer's attitude control system as it rejects the target's momentum. The rationalization of a multi arm servicer stems from the fact that in order to perform on-orbit servicing of the target, two or more arms will likely be required: one arm to grasp the target, and a second arm to perform on-orbit servicing.

4. The detumbling of a non-cooperative target using Multiple Mode Control should be investigated. Multiple Mode Control allows the manipulator's joints to work in passive or active mode. Joints in passive mode will exhibit passive like compliance allowing the manipulator to comply with unmodelled environmental constraints resulting in reduced constraint force/torque at the interaction point between the manipulator and the environment. This work has been started in [48], in which a selection criteria is presented to select manipulator joints to work in passive mode in order to reduce the momentum transfer from the servicer's end-effector to its base when interacting with an unknown object. This results in a reduced servicer's attitude control torque to maintain the servicer's base attitude when a force/torque is applied to the servicer's end-effector. 


\section{Appendix A}

\section{Description of Servicer and Target Model Parameters Used in Numerical Simulations}

Here, the model parameters of the servicer and target used in the numerical simulations are presented.

\section{A.1 Model Parameters for Servicer}

The numerical simulation study was carried out using a MATLAB Simulink add-on, SimMechanics [1]. SimMechanics allows the representation of a connected multibody system, such as the servicer, by connected individual blocks. Each individual block represents a single rigid body of the multi body system. The block are connected utilizing different joint connections blocks. The joint connection blocks impose kinematic constraints between the two bodies that determine how they can move relative to each other. Each joint has an actuation input that is determined by the detumbling control strategy.

The servicer consist of a base satellite and a 7 degree-of-freedom (DOF) manipulator. Each link of the manipulator is modelled as a rigid body and is connected to the lower link by a revolute joint (Fig. A.1). The first manipulator link is connected to the base by a revolute joint. The servicer's base center of mass is connected to the inertial frame by a 6-DOF joint. 

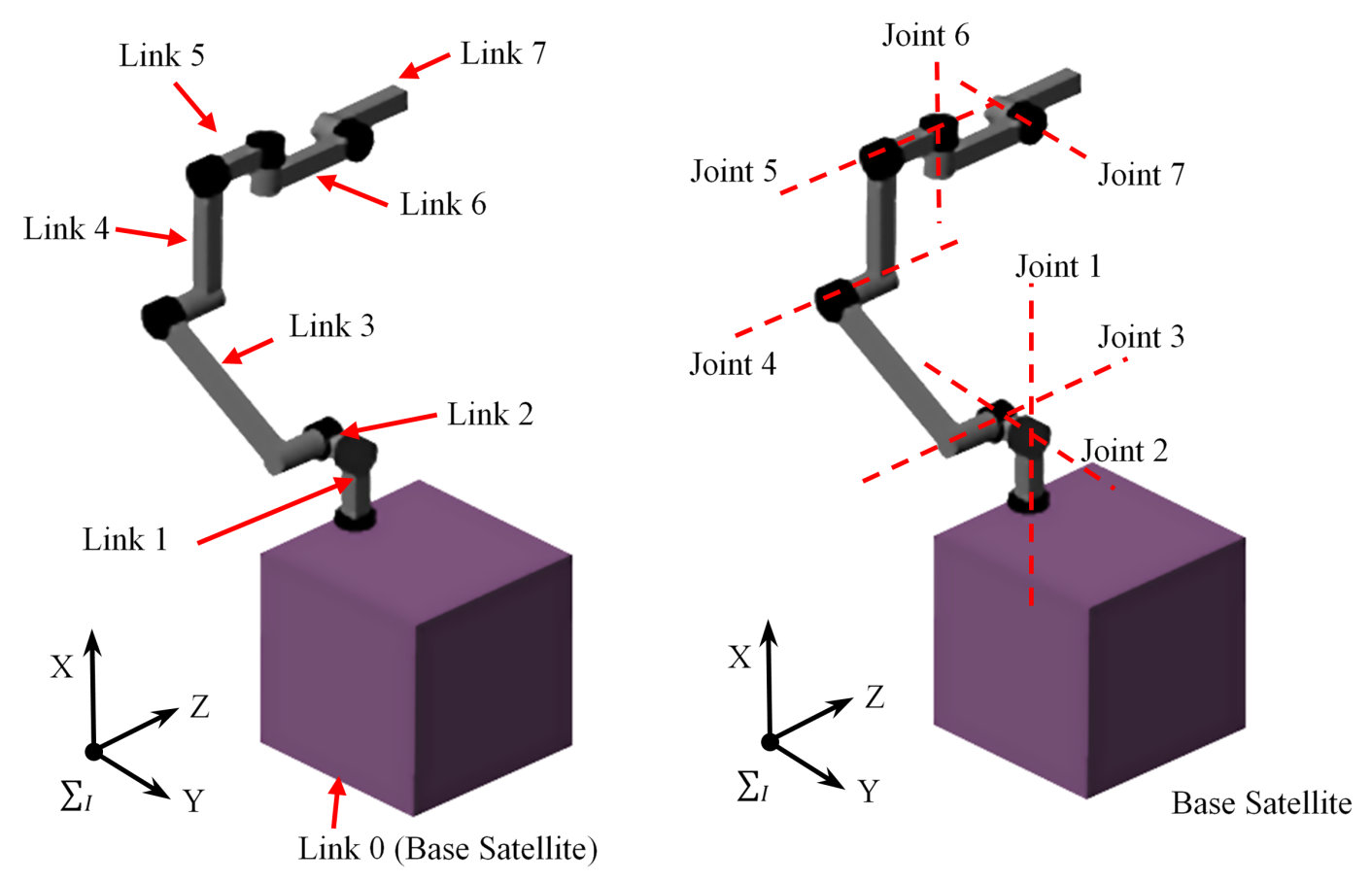

Figure A.1: Servicer model created in SimMechanics

The 6-DOF joint can accept both force and torque as actuation inputs. However, only actuation torques are applied to the 6-DOF joint. These actuation torques are determined from the detumbling control strategy.

As mentioned above, the servicer consist of a base satellite and a 7-DOF manipulator. The structure of the manipulator is depicted in Fig. A.1. The servicer's base is model as cube with $1 \mathrm{~m}$ sides. The 7-DOF manipulator is based on a modified model of the manipulator on Engineering Test Satellite Number 7 (ETS-VII) presented in [44]. Model parameters of the servicer are presented in Table A.1 and Denavit-Hartenberg parameters are presented in Table A.2 from [44]. 
Table A.1: Servicer's Model Parameters

\begin{tabular}{ccccc} 
& Mass $(\mathrm{kg})$ & $\mathrm{I}_{x x}\left(\mathrm{kgm}^{2}\right)$ & $\mathrm{I}_{y y}\left(\mathrm{kgm}^{2}\right)$ & $\mathrm{I}_{z z}\left(\mathrm{kgm}^{2}\right)$ \\
\hline Base & 1000 & 1200 & 1200 & 1200 \\
Link 1 & 35.01 & 1.218 & 0.5132 & 1.331 \\
Link 2 & 30 & 2.10 & 1.378 & 2.359 \\
Link 3 & 22.69 & 0.102 & 3.378 & 3.359 \\
Link 4 & 21.38 & 0.4327 & 2.266 & 1.911 \\
Link 5 & 16.75 & 0.3878 & 0.3963 & 0.07271 \\
Link 6 & 26.17 & 0.5727 & 0.5987 & 0.1288 \\
Link 7 & 18.07 & 0.165 & 0.241 & 0.135 \\
\hline
\end{tabular}

Table A.2: Denavit-Hatenberg table of 7-DOF manipulator

\begin{tabular}{ccccc} 
& $\mathrm{a}_{i}(\mathrm{~m})$ & $\alpha_{i}(\mathrm{deg})$ & $\mathrm{d}_{i}(\mathrm{~m})$ & $\phi_{i}(\mathrm{deg})$ \\
\hline Link 1 & 0 & 90 & 0.35 & $\phi_{1}$ \\
Link 2 & 0 & 90 & 0.2 & $\phi_{2}+90$ \\
Link 3 & 0.87 & 0 & 0.275 & $\phi_{3}$ \\
Link 4 & 0.63 & 0 & -0.275 & $\phi_{4}$ \\
Link 5 & 0 & 90 & -0.36 & $\phi_{5}-90$ \\
Link 6 & 0.55 & 90 & 0.16 & $\phi_{6}-90$ \\
Link 7 & 0.532 & 00 & 0.2 & $\phi_{7}$ \\
\hline
\end{tabular}

\section{A.2 Model Parameters for Target}

In the numerical simulation studies of this dissertation, the target is modelled as a rigid body. The target's inertial parameters such as its mass $\left(m_{\mathfrak{t}}\right)$, inertia tensor $\left(\mathbf{I}_{\mathfrak{t}}\right)$ and location of center of mass from the grasping location $\left(\mathbf{r}_{\text {te }}\right)$ are determined by the following steps.

1. The total mass of the target satellite is selected. The total mass of the satellite is denoted as $m_{\text {Ttotal }}$ and is the summation of the target's dry mass, $m_{\text {dry }}$, and the mass of the maximum amount of fuel that the target can hold at launch, $m_{\text {Mfuel }}$.

2. The spacecraft density, $\rho$, will vary between $\rho=20 \mathrm{~kg} / \mathrm{m}^{3}$ and $\rho=172 \mathrm{~kg} / \mathrm{m}^{3}$ with the average being $\rho=79 \mathrm{~kg} / \mathrm{m}^{3}$. This was obtained from [36] and reflects the density of the 75 spacecrafts launched between 1975 and 1984 in launch configuration with propellant loaded. With the use of the target's total mass and density, the volume of the satellite can be determined. The body of most satellites will be similar in shape to a cube or 
Table A.3: Target Properties for Numerical Simulation Studies

\begin{tabular}{|c|c|c|}
\hline & Section 3.4 .7 & Section: 4.2 \\
\hline$m_{\text {Ttotal }}(\mathrm{kg})$ & 500 & 170 \\
\hline$m_{\text {dry }}(\mathrm{kg})$ & 350 & 119 \\
\hline$m_{\text {Mfuel }}(\mathrm{kg})$ & 150 & 51 \\
\hline$s_{\text {side }}(\mathrm{m})$ & 1.95 & 1.3 \\
\hline$r_{\text {tank }}(\mathrm{m})$ & 0.45 & 0.3 \\
\hline
\end{tabular}

cylinder. Assuming the shape of the target is that of a cube, the dimensions of the cube can be estimated using the estimated volume. Let the length of the cube be denoted by $s_{\text {side }}$. The procedure utilized here to determine the size of the target satellite parallels that presented in [36] in estimating the size of the satellite based on its total mass.

3. As stated above, the total mass is the summation of its dry mass and total fuel mass: $m_{\text {Ttotal }}=m_{\text {dry }}+m_{\text {Mfuel }}$. From [36], the fuel mass of some satellite can be as high as $50 \%$ of their total mass. However the percentage of $m_{\text {Mfuel }}$ out of $m_{\text {Ttotal }}$ ultimately depends on the target satellite's mission. Herein, $m_{\text {Mfuel }}$ is selected as $30 \%$ of $m_{\text {Ttotal }}$. Furthermore, an assumed size of the fuel tank is made. As per Fig. A.2 the fuel tank is assumed to sit just below the target's geometric center, with the aim of keeping the tank close to the target's center of mass. From Fig. A.2, the fuel tank is modelled as the smaller cube that sits inside of the larger cube, that is the target. The length of the smaller cube (tank) is denoted by $r_{\text {tank }}$. For the numerical simulations carried out in Section 3.4.7 and 4.2, the assumed mass and size of the target satellite and fuel tanks are presented in Table A.3.

4. Making use of the mass properties and geometric properties of the satellite body and of its tank in Table A.3, the inertia tensor of the satellite with zero fuel and with a full tank of fuel about the satellite's body geometric center can be determined and are denoted as $\mathbf{I}_{\text {tarDRY }}$ and $\mathbf{I}_{\text {tarWET }}$, respectively. The target's center of mass with respect to frame $\Sigma_{\mathrm{t}}$ in Fig. A.2 can be determined with and with out fuel and are denoted as $\mathbf{r}_{\mathrm{cgDRY}}$ and $\mathbf{r}_{\mathrm{cgWET}}$, respectively. The origin of $\Sigma_{\mathrm{t}}$ in Fig. A.2 is located at the geometric center of the target's cube shape body. These parameters are presented in Table A.4.

The numerical simulations in Section 3.4.7 and 4.2 require the upper and lower bounds on the target's inertial parameters. The upper bounds on the target's inertial parameters are 
Table A.4: Target Inertia Tensor and Location of Center of Mass

\begin{tabular}{ccc} 
& Section $\sqrt[3.4 .7]{ }$ & Section: 4.2 \\
\hline $\mathbf{I}_{\text {tarDRY }},\left(\mathrm{kgm}^{2}\right)$ & $\operatorname{diag}([212.8,212.8,219.8])$ & $\operatorname{diag}([32.2,32.2,33.2])$ \\
$\mathbf{I}_{\text {tarWET }},\left(\mathrm{kgm}^{2}\right)$ & $\operatorname{diag}([263.4,263.4,240.1])$ & $\operatorname{diag}([39.8,39.8,36.3])$ \\
$\mathbf{r}_{\text {cgDRY }},(\mathrm{m})$ & {$[0,0,0.12]^{\mathrm{T}}$} & {$[0,0,0.08]^{\mathrm{T}}$} \\
$\mathbf{r}_{\text {cgWET }},(\mathrm{m})$ & {$[0,0,-0.05]^{\mathrm{T}}$} & {$[0,0,-0.04]^{\mathrm{T}}$} \\
\hline
\end{tabular}

determined by multiplying the target's dry mass and max fuel mass by $1.2(20 \%)$ to determine the mass, inertia tensor and location of center of mass. The lower bound is determined by multiplying the target's dry mass by 0.8 and setting the fuel mass to zero to determine the mass, inertia tensor and location of center of mass of the target. In the calculation of bounds on the target's inertial parameters, the size of the target's body and fuel tank are assumed fixed. An uncertainty of $20 \%$ is utilized to reflect the uncertainty associated with mass estimation of a satellite's inertial properties prior to launch [15]. These values are presented in Table A.5 and A.6 for the targets in utilized in Section 3.4.7 and 4.2, respectively.

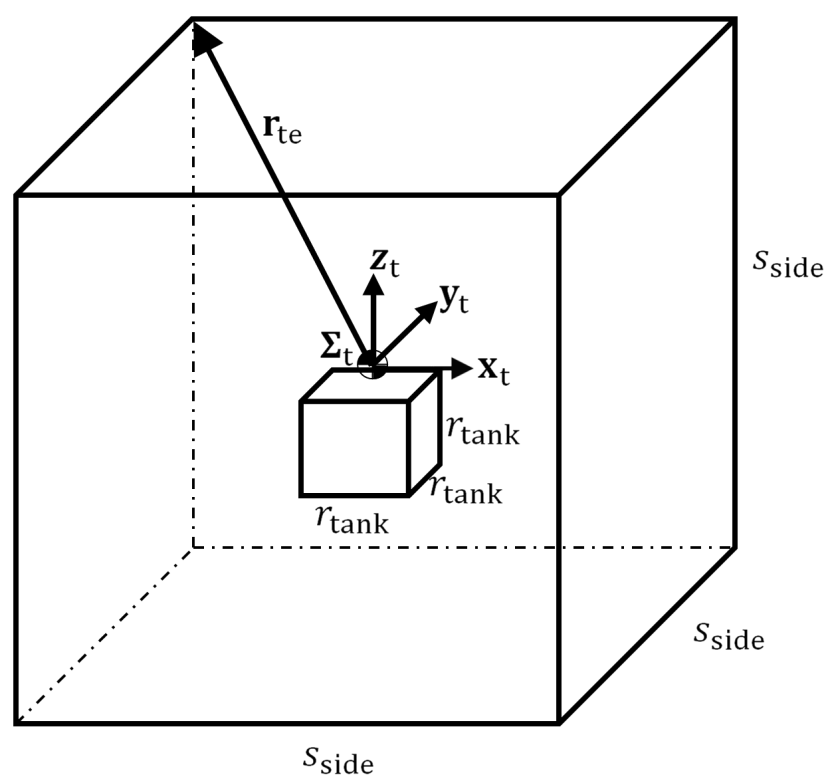

Figure A.2: Target model. The origin of $\Sigma_{\mathrm{t}}$ coincides with the geometric center of the target's satellite body. 
Table A.5: Bounds on Target's Inertial Parameters for Section 3.4.7

\begin{tabular}{ccc} 
& Lower Bound & Upper Bound \\
\hline$m_{\mathrm{t}}(\mathrm{kg})$ & 280 & 600 \\
$\mathbf{I}_{\mathbf{t}}\left(\mathrm{kgm}^{2}\right)$ & $\operatorname{diag}([170.3,170.3,175.9])$ & $\operatorname{diag}([316.1,316.1,288.1])$ \\
$\left\|\mathbf{r}_{\mathrm{te}}\right\|(\mathrm{m})$ & 1.62 & 1.71 \\
\hline
\end{tabular}

Table A.6: Bounds on Target's Inertial Parameters for Section 4.2

\begin{tabular}{ccc} 
& Lower Bound & Upper Bound \\
\hline$m_{\mathrm{t}}(\mathrm{kg})$ & 95.2 & 204 \\
$\mathbf{I}_{\mathbf{t}}\left(\mathrm{kgm}^{2}\right)$ & $\operatorname{diag}([25.7,25.7,26.5])$ & $\operatorname{diag}([47.8,47.8,43.5])$ \\
$\left\|\mathbf{r}_{\mathrm{te}}\right\|(\mathrm{m})$ & 1.08 & 1.15 \\
\hline
\end{tabular}




\section{Appendix B}

\section{Upper Bound on $\|\chi\|$ and $\left\|\dot{f}(\mathbf{V})^{\mathrm{d}}\right\|$}

Here, it is demonstrated that $\chi$ and $\dot{f}(\mathbf{V})^{\mathrm{d}}$ are upper bounded by a finite positive constant provided that $\left(\mathbf{s}_{1}, \mathbf{s}_{2}\right) \in \Omega_{2}$ and the desired velocity and its first two derivatives are contained in the set $\Omega_{1}$. From this, it will become clear why $\chi$ and $\dot{f}(\mathbf{V})^{\mathrm{d}}$ cannot be bounded if the servicer's manipulator is redundant with respect to the end-effector's task space representation.

In order to conclude boundedness of $\mathbf{s}_{1}$ and $\mathbf{s}_{2}$, it is required that $\chi$ and $\dot{f}(\mathbf{V})^{\mathrm{d}}$ be bounded. From Chapter 4.1.3, the definition of $\chi$ and $\dot{f}(\mathbf{V})^{\mathrm{d}}$ are presented below:

$$
\begin{gathered}
\chi=\overline{\mathbf{M}}_{\mathrm{o}}^{-1}\left(\mathbf{A}^{\mathrm{T}} \mathbf{s}_{2}-\mathbf{J}_{\mathrm{s}} \gamma_{2}-\Delta \overline{\mathbf{M}} \dot{\xi}\right) \\
\dot{f}(\mathbf{V})^{\mathrm{d}}=\dot{\mathbf{A}}^{-\mathrm{T}}\left(\overline{\mathbf{c}}_{\mathrm{o}}+\overline{\mathbf{M}}_{\mathrm{o}} \dot{\xi}^{\mathrm{d}}-\overline{\mathbf{M}}_{\mathrm{o}} \mathbf{K}_{\mathrm{P}} \dot{\mathbf{e}}_{\mathrm{P}}-\overline{\mathbf{M}}_{\mathrm{o}} \mathrm{K}_{1} \tanh \left(\mathbf{s}_{1}\right)-\psi_{\mathrm{P} 1}\right)+ \\
\mathbf{A}^{-\mathrm{T}}\left(\dot{\overline{\mathbf{c}}}_{\mathrm{o}}+\frac{d}{d t}\left(\overline{\mathbf{M}}_{\mathrm{o}} \dot{\xi}^{\mathrm{d}}-\overline{\mathbf{M}}_{\mathrm{o}} \mathbf{K}_{\mathrm{P}} \dot{\mathbf{e}}_{\mathrm{P}}-\overline{\mathbf{M}}_{\mathrm{o}} \mathrm{K}_{1} \tanh \left(\mathbf{s}_{1}\right)-\psi_{\mathrm{P} 1}\right)\right)
\end{gathered}
$$

From (1) and (2), boundedness of $\chi$ and $\dot{f}(\mathbf{V})^{\mathrm{d}}$ can be concluded if the terms on the right side of (1) and (2) are bounded. Consider the following remarks:

Remark 1: $\overline{\mathbf{c}}_{\mathrm{o}}$ represents the non-linear velocity dependent terms and is a function of the servicer's base linear and angular velocity $\left(\boldsymbol{v}_{\mathrm{b}}\right)$ and servicer's manipulator joint velocity $\left(\dot{\phi}_{\mathrm{m}}\right)$. $\gamma_{2}$ and $\dot{\mathbf{e}}_{\mathrm{p}}$ are functions of $\boldsymbol{v}_{\mathrm{e}}$ and $\mathbf{w}_{\mathrm{b}}$

Remark 2: $\dot{\overline{\mathbf{c}}}_{\mathrm{o}}$ is a function of $\boldsymbol{v}_{\mathrm{b}}, \dot{\phi}_{\mathrm{m}}$ and their derivatives $\left(\dot{\boldsymbol{v}}_{\mathrm{b}}, \ddot{\phi}_{\mathrm{m}}\right)$.

Remark 3: A, $\overline{\mathbf{M}}_{\mathrm{o}}, \mathbf{J}_{\mathrm{s}}$ and $\Delta \overline{\mathbf{M}}$ are functions of the servicer's configuration (dependent 
on position and orientation of the servicer's base and manipulator), its inertial and geometric properties as well as the target's inertial parameters and are bounded.

Remark 4: The derivative of $\mathbf{A}$ and $\overline{\mathbf{M}}_{\mathrm{o}}$ with respect to time are a function of the servicer's configuration, inertial properties and $v_{\mathrm{b}}, \dot{\phi}_{\mathrm{m}}$.

Remark 5: $\psi_{\mathrm{P} 1}$ (defined in equation (4.34) ) is bounded. The derivative of $\psi_{1}$ and $\mathbf{s}_{1}$ with respect to time are a function of the servicer's configuration, $v_{\mathrm{b}}, \dot{\phi}_{\mathrm{m}}, \dot{\boldsymbol{v}}_{\mathrm{b}}$ and $\ddot{\phi}_{\mathrm{m}}$.

For $\chi$ and $\dot{f}(\mathbf{V})^{\mathrm{d}}$ to be bounded, it is required $\boldsymbol{v}_{\mathrm{e}}, \boldsymbol{v}_{\mathrm{b}}, \dot{\phi}_{\mathrm{m}}, \dot{\boldsymbol{v}}_{\mathrm{e}}, \dot{\boldsymbol{v}}_{\mathrm{b}}$ and $\ddot{\phi}_{\mathrm{m}}$ be bounded.

Assumption A1: It is assumed that $\left(\mathbf{s}_{1}, \mathbf{s}_{2}\right) \in \Omega_{2}$. Recall that $\xi=\left[\boldsymbol{v}_{\mathrm{e}}^{\mathrm{T}}, \mathbf{w}_{\mathrm{b}}^{\mathrm{T}}\right]^{\mathrm{T}}$. It is assumed that the desired end-effector position trajectory as well as $\xi^{\mathrm{d}}$ and its first two derivatives are contained in the set $\Omega_{1}$.

Remark 6: Corollary 1 (Chapter 2.1), demonstrates that the servicer's end-effector position is bounded.

Boundedness of $\boldsymbol{v}_{\mathrm{e}}, \boldsymbol{v}_{\mathrm{b}}, \dot{\phi}_{\mathrm{m}}, \dot{\boldsymbol{v}}_{\mathrm{e}}, \dot{\boldsymbol{v}}_{\mathrm{b}}$ and $\ddot{\phi}_{\mathrm{m}}$ is demonstrated as follows:

Step 1: Recalling the definition of $\mathbf{s}_{1}$ 4.22, and using Assumption A1 and Remark 6, we can conclude that $\xi=\left[v_{\mathrm{e}}^{\mathrm{T}}, \mathbf{w}_{\mathrm{b}}^{\mathrm{T}}\right]^{\mathrm{T}}$ is bounded.

Step 2: Equation 2.29) is rewritten here for convenience as follows:

$$
\boldsymbol{v}_{\mathrm{e}}=\mathbf{A}_{\mathrm{b}} \mathbf{w}_{\mathrm{b}}+\mathbf{A}_{\mathrm{m}} \dot{\phi}_{\mathrm{m}}+\frac{1}{m_{\mathrm{total}}} \mathbf{P}_{\mathrm{b}}
$$

where the definition of $\mathbf{A}_{\mathrm{b}} \in \mathbb{R}^{6 \times 3}$ and $\mathbf{A}_{\mathrm{m}} \in \mathbb{R}^{6 \times m}$ are defined as 2.30) and 2.31), respectively $\left(\mathbf{A}_{\mathrm{m}}\right.$ and its inverse are functions of the servicer's configuration and are bounded). $\mathbf{P}_{\mathrm{b}}$ is the linear momentum of the combined system (servicer/target) and is constant under the assumption that there is no external force/torque applied to the servicer/target system. Equation $(B .3)$ is different from (2.29) because the matrices in $\mathrm{B} .3$ ) are determined using mass properties (mass, inertia tensor, center of mass) based on the combined servicer/target system. Recalling that $v_{\mathrm{e}}$ and $\mathbf{w}_{\mathrm{b}}$ are bounded from Step 1, it follows from (B.3) that the manipulator's joint velocities, $\dot{\phi}_{\mathrm{m}}$, are bounded if $\mathbf{A}_{\mathrm{m}}$ is square and $\operatorname{rank}\left(\mathbf{A}_{\mathrm{m}}\right)=6$.

By forcing $\mathbf{A}_{\mathrm{m}}$ to be square and $\operatorname{rank}\left(\mathbf{A}_{\mathrm{m}}\right)=6$, we are removing any redundancy in the manipulator with respect to the end-effector's task space representation. If the manipulator is to possess any redundancy with respect to its end-effector's task space representation, then it would not be possible to conclude boundedness of $\dot{\phi}_{\mathrm{m}}$ from B.3. This can be observed by 
solving for $\dot{\phi}_{\mathrm{m}}$ from $\left.\mathrm{B} .3\right)$ as follows:

$$
\dot{\phi}_{\mathrm{m}}=\mathbf{A}_{\mathrm{m}}^{+}\left(\boldsymbol{v}_{\mathrm{e}}-\mathbf{A}_{\mathrm{b}} \mathbf{w}_{\mathrm{b}}-\frac{1}{m_{\text {total }}} \mathbf{P}_{\mathrm{b}}\right)+\dot{\phi}_{\mathrm{mNL}}
$$

where $\dot{\phi}_{\mathrm{mNL}}$ is a component of the manipulator's joint velocity that lies in the null space of $\mathbf{A}_{\mathrm{m}}$. The first terms, $\mathbf{A}_{\mathrm{m}}^{+}\left(\boldsymbol{v}_{\mathrm{e}}-\mathbf{A}_{\mathrm{b}} \mathbf{w}_{\mathrm{b}}-\frac{1}{m_{\text {total }}} \mathbf{P}_{\mathrm{b}}\right)$ can be bounded but boundedness of $\dot{\phi}_{\mathrm{mNL}}$ cannot be assumed.

Step 3: Equation (2.28) is restated here for convenience:

$$
v_{\mathrm{e}}=\mathbf{J}_{\mathrm{b}} \boldsymbol{v}_{\mathrm{b}}+\mathbf{J}_{\mathrm{m}} \dot{\phi}_{\mathrm{m}}
$$

where $\mathbf{J}_{\mathrm{b}} \in \mathbb{R}^{6 \times 6}$ and $\mathbf{J}_{\mathrm{m}} \in \mathbb{R}^{6 \times m}$ are both square and full rank. $\mathbf{J}_{\mathrm{b}}$ and its inverse are functions of the servicer's configuration and are bounded. Provided that $v_{\mathrm{e}}$ and $\dot{\phi}_{\mathrm{m}}$ are bounded from Steps 1 and 2, it follows that $\boldsymbol{v}_{\mathrm{b}}$ is bounded from (B.5).

Step 4: Equation 2.23) is rewritten here for convenience::

$$
\mathbf{H}_{\gamma}\left[\begin{array}{c}
\dot{\boldsymbol{v}}_{\mathrm{b}} \\
\ddot{\boldsymbol{\phi}}_{\mathrm{m}}
\end{array}\right]+\mathbf{c}_{\gamma}=\left[\begin{array}{c}
\mathbf{f}_{\mathrm{b}} \\
\tau_{\mathrm{m}}
\end{array}\right]+\left[\begin{array}{c}
\mathbf{J}_{\mathrm{b}}^{\mathrm{T}} \\
\mathbf{J}_{\mathrm{m}}^{\mathrm{T}}
\end{array}\right]\left(-\mathbf{f}_{\mathrm{e}}\right)
$$

If the mass properties of the target are considered an extension of the servicer's manipulator and under the assumption that there is no external force on the combined servicer/target system $\left(\mathbf{F}_{\mathrm{b}}=0\right)$, B.6 becomes:

$$
\mathbf{H}_{\mathrm{fl}}\left[\begin{array}{c}
\dot{\mathbf{v}}_{\mathrm{b}} \\
\dot{\mathbf{w}}_{\mathrm{b}} \\
\ddot{\phi}_{\mathrm{m}}
\end{array}\right]+\mathbf{c}_{\mathrm{fl}}=\left[\begin{array}{c}
0 \\
\tau_{\mathrm{b}} \\
\tau_{\mathrm{m}}
\end{array}\right]
$$

where the inertial properties of the manipulator's last link are modified to reflect the rigidly attached target to the end-effector and $\mathbf{H}_{\gamma}$ is positive definite. $\tau=\left[\tau_{\mathrm{b}}^{\mathrm{T}}, \tau_{\mathrm{m}}^{\mathrm{T}}\right]=f(\mathbf{V})$ is bounded as per $4.24 \mathrm{~b}$. The nonlinear velocity dependent term, $\mathbf{c}_{\gamma}$, is bounded since $\boldsymbol{v}_{\mathrm{e}}, \boldsymbol{v}_{\mathrm{b}}$ and $\dot{\phi}_{\mathrm{m}}$ are all bounded from Steps 1 to 3. It follows from (B.7) that $\dot{\mathbf{v}}_{\mathrm{b}}, \dot{\mathbf{w}}_{\mathrm{b}}$ and $\ddot{\phi}_{\mathrm{m}}$ are bounded. Furthermore, from the time derivative of the kinematic equation (B.5), it follows that $\dot{\boldsymbol{v}}_{\mathrm{e}}$ is also bounded.

With the use of Assumption A1, Steps 1 to 4 demonstrated the boundedness of $v_{\mathrm{e}}, \boldsymbol{v}_{\mathrm{b}}, \dot{\phi}_{\mathrm{m}}$, $\dot{\boldsymbol{v}}_{\mathrm{e}}, \dot{\boldsymbol{v}}_{\mathrm{b}}$ and $\ddot{\phi}_{\mathrm{m}}$. As a result there exist an upper bound on $\chi$ and $\dot{f}(\mathbf{V})^{\mathrm{d}}$ such that $\|\chi\| \leq \mathrm{M}_{1}$ and $\left\|\dot{f}(\mathbf{V})^{\mathrm{d}}\right\| \leq \mathrm{M}_{2}$ where $\mathrm{M}_{i=1,2}$ are positive constants. 


\section{References}

[1] MATLAB and SimMechanics Toolbox release 2016a. The MathWorks, Inc., Natick, Massachusetts, United States, 2016.

[2] S. Abiko and G. Hirzinger. On-line parameter adaptation for a momentum control in the post-grasping of a tumbling target with model uncertainty. In Proc. of IEEE/RSJ International Conference on Intelligent Robots and Systems, pages 847-852, San Diego, CA, 2007.

[3] S. Abiko, R. Lampariello, and G. Hirzinger. Impedance control for a free-floating robot in the grasping of a tumbling target with parameter uncertainty. In Proc. of IEEE/RSJ International Conference on Intelligent Robots and Systems, pages 1020-1025, Beijing, 2006.

[4] S. Abiko and K. Yoshida. Adaptive reaction control for space robotic application with dynamic model uncertainty. Advanced Robotics, 24(8-9):1099-1126, 2010.

[5] F. Aghili. Optimal control for robotic capturing and passivation of a tumbling satellite with unknown dynamics. In AIAA Guidance, Navigation and Control Conference, Beijing, 2006. AIAA.

[6] F. Aghili. Coordination control of a free-fying manipulator and its base attitude to capture and detumble a non-cooperative satellite. In Proc. of IEEE/RSJ International Conference on Intelligent Robots and Systems, pages 2365-2372, 2009.

[7] F. Aghili. Optimal control of a space manipulator for detumbling of a target satellite. In Proc. of IEEE/RSJ International Conference on Intelligence Robots and Automation, pages 3019-3024, Kobe, Japan, 2009. 
[8] F. Aghili. A prediction and motion-planning scheme for visually guided robotic capturing of free-floating tumbling objects with uncertain dynamics. IEEE Transactions on Robotics, 28(3):634-649, 2012.

[9] F. Aghili. Pre and post-grasping robot motion planning to capture and stabilize a tumbling/drifting free-floater with uncertain dynamics. In Proc. of IEEE/RSJ International Conference on Intelligent Robots and Automation, pages 5461-5468, Karlsruhe, Germany, 2013.

[10] F. Aghili and K. Parsa. Motion and parameter estimation of space object using laservision data. Journal of Guidance, Control, and Dynamics, 32(2):538-550, 2009.

[11] R.J. Anderson and M.W. Spong. Asymptotic stability for force reflecting teleoperators with time delay. The International Journal of Robotics Research, 11(2):135-149, 1991.

[12] A. Antonello, A. Valverde, and P. Tsiotras. Dynamics and control of spacecraft manipulators with thrusters and momentum exchange devices. Journal of Guidance, Control, and Dynamics, 42(1):15-29, 2019.

[13] B. Bischof, L. Kerstein, J. Starke, H. Guenther, and W.P. Foth. Roger-robotic geostationary orbit restorer. In 54th International Astronautical Federation, the International Academy of Astronautics, and the International Institute of Space Law, Bremen, Germany, 2003.

[14] F. Caccavale and B. Siciliano. Kinematic control of redundant free-floating robotic systems. Advanced Robotics, 15(4):429-448, 2001.

[15] A. Chuchra, G. Sebestyen, N. Galassi, and S. Fujikawa. Low earth orbit satellite design. Springer, 2018.

[16] A.H.J. de Ruiter. Spacecraft attitude tracking with guaranteed performance bounds. Journal of Guidance, Control, and Dynamics, 36(4):1214-1221, 2013.

[17] M. de Stefano, H. Mishra, R. Balachandran, R. Lampariello, C. Ott, and C. Secchi. Multirate tracking control for a space robot on a controller satellite: a passivity-based strategy. IEEE Robotics and Automation Letters, 2019. 
[18] D.N. Dimitrov and Y. Yoshida. Momentum distribution in a space manipulator for facilitating the post-impact control. In Proc. of IEEE/RSJ International Conference on Intelligent Robots and Systems, pages 3345-3350, Sendai, Japan, 2004.

[19] G. Dong and Z.H. Zhu. Incremental inverse kinematics based vision servo for autonomous robotic capture of non-cooperative space debris. Advances in Space Research, 57(7):1508-1514, 2016.

[20] M.A. Estrada, H. Jiang, B. Noll, E.W. Hawkes, M. Pavone, and M.R. Cutkosky. Force and moment constraints of a cured surface gripper and wrist for assistive free flyers. In Proc. of IEEE International Conference on Robotics and Automation, pages 2824-2830, Singapore, 2017.

[21] X. Feng, Y. Jia, and S. Xu. Inertia-free minimum-angular-motion detumbling control of spacecraft. Journal of Guidance, Control, and Dynamics, 42(3):678-683, 2019.

[22] A. Flores-Abad and O. Ma. A review of space robotics technologies for on-orbit servicing. Progress in Aerospace Science, 68:1-26, 2014.

[23] R.B. Friend. Orbital express program summary and mission overview. Sensors and Systems for Space Applications II, 6958, 2008.

[24] H. Gui and A.H.J. de Ruiter. Adatpive fault-tolerant spacecraft pose tracking with control allocation. IEEE Transaction on Control Systems Technology, 2017.

[25] H. Gui and A.H.J. de Ruiter. Control of astroid-hovering spacecraft with disturbance rejection using position-only measurements. Journal of Guidance Control and Dynamics., 40(10):2401-2416, 2017.

[26] H. Heidari, M.J. Pouria, S. Sharifi, and M. Karami. Design and fabrication of robotic gripper for grasping in minimizing contact force. Advances in Space Research, 61(5):13591370, 2018.

[27] G. Hirzinger, K. Landzettel, B. Brunner, M. Fischer, C. Preusche, D. Reintsema, A. AlbuSchäffer, G. Schreiber, and B.M. Steinmetz. DLR's robotics technologies for on-orbit servicing. Advanced Robotics, 18(2):139-174, 2004. 
[28] Z.-G. Hou, A.-M. Cheng, and M. Tan. Adaptive control of an electrically driven nonholonomic mobile robot via backstepping and fuzzy approach. IEEE Transactions on Control Systems Technology, 17(4):803-815, 2009.

[29] H. S. Jayakody, L. Shi, J. Katupitiya, and N. Kinkaid. Robust adaptive coordination controller for a spacecraft equipped with a robotic manipulator. Journal of Guidance, Control, and Dynamics, 39(12):2699-2710, 2016.

[30] S. Jia and J. Shan. Finite-time trajectory tracking control of space manipulator under actuator saturation. IEEE Transactions on Industrial Electronics, 2019.

[31] H. K. Khalil. Nonlinear systems. Prentice-Hall., New Jersey, 1992.

[32] R. Lampariello. Motion planning for the on-orbit grasping of a non-cooperative target satellite with collision avoidance. In Prco. of the 10th International Symposium on Artificial Intelligence, Robotics and Automation in Space, Sapporo, Japan, 2010. i-SAIRAS.

[33] R. Lampariello. On grasping a tumbling debris object with a free-flying robot. In IFAC Proceedings, pages 161-166, 2013.

[34] R. Lampariello and G. Hirzinger. Generating feasible trajectories for autonomous onorbit grasping of spinning debris in a useful time. In Proc. of the IEEE/RSJ International Conference on Intelligent Robots and Systems, pages 5652-5659, Tokyo, Japan, 2013. IEEE.

[35] R. Lampariello, H. Mishra, N. Oumer, P. Schmidt, and M. De Stefano. Tracking control for the grasping of a tumbling satellite with a free-floating robot. IEEE Robotics and Automation Letters, 3(4):3638-3645, 2018.

[36] W.J. Larson and J.R. Wertz. Space mission analysis and design. Microcosm Press, CA, United States, 1992.

[37] O. Ma, H. Dang, and K. Pham. On-orbit identification of inertia properties of spacecraft using a robotic arm. Journal of Guidance Control and Dynamics, 32(6):1761-1771, 2008.

[38] O. Ma and E. Martin. Extending the capability of attitude control systems to assist satellite docking missions. In Proceedings CCToMM Symposium on Mechanisms, Machines, and Mechatronics, 2001. 
[39] E. Magrini, F. Flacco, and A. De Luca. Control of generalized contact motion and force in physical human robot interaction. In Prco. of the IEEE International Conference on Robotics and Automation, pages 2298-2304, Seattle, WA, 2015. IEEE.

[40] D. Mehrholz, L. Leushacke, W. Flury, R. Jehn, H. Klinkrad, and M. Landgraf. Detecting tracking and imaging space debris. ESA Bulletin, 109:128-134, 2002.

[41] Y. Murotsu, K. Senda, M. Ozaki, and S. Tsuhio. Parameter identification of unknown object handled by free-flying space robot. Journal of Guidance Control and Dynamics, 17(3):488-494, 1994.

[42] D.N. Nechev and K. Yoshida. Impact analysis and post-impact control issues of a free floating space robot subject to a force impulse. IEEE Transactions on Robotics and Automation, 15(3):548-557, 1999.

[43] L.K. Newman. The nasa robotic conjunction assessment process: overview and operational experiences. Acta Astronautica, 66:1253-1261, 2010.

[44] T.C. Nguyen-Huynh and I. Sharf. Adaptive reactionless motion and parameter identification in postcapture of space debris. Journal of Guidance Control and Dynamics., 36(2):404-414, 2013.

[45] M. Oda, K. Kibe, and F. Yamagata. Ets-vii, space robot in-orbit experiment satellite. In Proc. of IEEE International Conference on Robotics and Automation, pages 739-744, Minneapolis, USA, 1996.

[46] A. Ogilvie, J. Allport, M. Hannah, and J. Lymer. Autonomous satellite servicing using the orbital express demonstration manipulator system. In Prco. of the 9th International Symposium on Artificial Intelligence, Robotics and Automation in Space, Hollywood, USA, 2008. i-SAIRAS.

[47] T. Oki, S. Abiko, H. Nakanishi, and K. Yoshida. Time-optimal detumbling maneuver along an arbitrary arm motion during the capture of a target satellite. In Prco. of IEEE/RSJ International Conference on Intelligent Robots and Systems, pages 625-630, 2011.

[48] T. Pankov, R. Gangapersaud, and G. Liu. A multiple working mode approach to control space manipulator interaction with unknown targets. In The International Symposium on Artificial Intelligence, Robotics and Automation in Space, Beijing, China, 2016. 
[49] E. Papadopoulos. On the dynamics and control of space manipulators. PhD thesis, Dept. of Mech. Eng., MIT, Cambridge, October 1990.

[50] M. Pasand, A. Hassani, and M. Ghorbani. A study of spacecraft reaction thruster configuration for attitude control system. IEEE Aerospace and Electronic Systems Magazine, 32(7):22-39, 2017.

[51] P.R. Perez, M. De Stefano, and R. Lampariello. Velocity matching compliant control for a space robot during capture of a free-floating target. In IEEE Aerospace Conference, pages $1-9,2018$.

[52] M.M. Polycarpou and P.A. Ioannou. A robust adaptive nonlinear control design. Automatica, 32(3):423-427, 1996.

[53] I. Rekleitis, E. Martin, G. Roulean, R. LArchevłque, K. Parsa, and E. Dupuis. Autonomous capture of a tumbling satellite. Journal of Field Robotics, 24(4):275-296, 2007.

[54] T. Rybus, K. Seweryn, and J.K. Sasiadek. Optimal detumbling of defunct spacecraft using space robots. In Proc. of the IEEE 14th International Conference on Methods and Models in Automation and Robotics, pages 64-69, Miedzyzdroje, Poland, 2014.

[55] M. Shan, J. Guo, and E. Gill. Review and comparison of active space debris capturing and removal methods. Progress in Aerospace Sciences, 80:18-32, 2016.

[56] M.J. Sidi. Spacecraft dynamics and control: a practical engineering approach. Cambridge University Press, NY, United States, 1997.

[57] G. Song and R. Mukherjee. A comparative study of conventional nonsmooth timeinvariant and smooth time-varying robust compensator. IEEE Transactions on Control System Technology, 6(4):571-576, 1998.

[58] V. Utkin and H. Lee. Chattering problem in sliding mode control systems. In International Workshop on Variable Structure Systems, pages 346-350, 2006.

[59] N. Uyama, H. Nakanishi, K. Nagaoka, and K. Yoshida. Impedance-based contact control of a free-flying space robot with a compliant wrist for non-cooperative satellite capture. 
In Prco. of IEEE/RSJ International Conference on Intelligent Robots and Systems, pages 4477-4482, 2012.

[60] N. Uyama and T. Narumi. Hybrid impedance/position control of a free-flying space robot for detumbling a noncooperative satellite. IFAC-Papers OnLine, 49(17):230-235, 2016.

[61] Z. Vafa and S. Dubowsky. The kinematics and dynamics of space manipulators: The virtual manipulator approach. The International Journal of Robotics Research, 9(4):321, 1990.

[62] B. Bastida Virgili, S. Lemmens, and H. Krag. Investigation on envisat attitude motion. In Investigation on Envisat attitude motion. ESA/ESOC, 2014.

[63] B.B. Virgili, S. Lemmens, and H. Krag. Investigation on envisat attitude motion. In $e$. Deorbit Workshop, 2014.

[64] R. Volpe and P. Khosla. An experimental evaluation and comparison of explicit force control strategies for robotic manipulators. In Prco. of the IEEE International Conference on Robotics and Automation, pages 1387-1393, Nice, France, 1992. IEEE.

[65] M. Wang, J. Luo, J. Yuan, and U. Walter. Detumbling control for kinematically redundant space manipulator post-grasping a rotational satellite. Acta Astronautica, 141:98-109, 2017.

[66] M. Wang, J. Luo, J. Yuan, and U. Walter. An integrated control scheme for space robot after capturing non-cooperative target. Acta Astronautica, 147:350-363, 2018.

[67] C. Xavier and J.J. Gilber. Postcapture dynamics of a spacecraft-manipulator-payload system. Journal of Guidance Control and Dynamics, 23(1):95-100, 2000.

[68] Y. Xu and T. Kanade, editors. Space robotics: dynamics and control. Springer, Norwell, 1993.

[69] K. Yoshida. The spacedyn: a matlab toolbox for space and mobile robots. In Proc. of IEEE/RSJ International Conference on Intelligent Robots and Systems, pages 1633-1638, Kyongju, South Korea, 1999. 
[70] K. Yoshida. Engineering test satellite vii flight experiment for space robot dynamics and control theories on laboratory test beds ten years ago, not in orbit. International Journal of Robotics Research', 22(5):321-335, 2003.

[71] K. Yoshida, H. Nakanishi, H. Ueno, N. Inaba, T. Nishimaki, and M. Oda. Dynamics, control and impedance matching for robotic capture of a non-cooperative satellite. Advanced Robotics, 18(2):175-198, 2004.

[72] Y. Yoshida and D.N. Dimitrov. On the capture of tumbling satellite by a space robot. In Proc. of IEEE/RSJ International Conference on Intelligent Robots and Systems, pages 4127-4132, Beijing, 2006.

[73] J.S. Yuan. Closed-loop manipulator control using quaternion feedback. IEEE Transactions on Robotics and Automation, 4(4):434-440, 1988.

[74] B. Zhang, B. Liang, Z. Wang, Y. Mi, Y. Zhang, and Z. Chen. Coordinated stabilization for space robot after capturing a non-cooperative target with large inertia. Acta Astronautica, 134:75-84, 2017.

[75] B.-Z. Zhou, G.-P. Cai, Y.-M. Liu, and P. Liu. Motion prediction of a non-cooperative space target. Advances in Space Research, 61(1):207-222, 2018.

[76] Y. Zhu, J. Qiao, and L. Guo. Adaptive sliding mode disturbance observer-based composite control with prescribed performance of space manipulators for target capturing. IEEE Transactions on Industrial Electronics, 66(3):1973-1983, 2019.

[77] A.M Zou, K.D. Kumar, and A.H.J. de Ruiter. Robust attitude tracking control of spacecraft under control input magnitude and rate saturations. International Journal of Robust and Nonlinear Control, 26(4):799-815, 2016. 